\title{
The Lace Expansion and its Applications
}

Gordon Slade

Department of Mathematics, University of British Columbia, Vancouver, BC,

Canada V6T 1 Z2

slade@math.ubc.ca

Version 3 (Final), August 9, 2005

Lecture notes for the XXXIV ${ }^{\text {th }}$ Saint-Flour International Probability School, July 2004, and for the Pacific Institute for the Mathematical Sciences - University of British Columbia Summer School on Probability, June 2005. 
The Lace Expansion and its Applications 


\section{Preface}

Several superficially simple mathematical models, such as the self-avoiding walk and percolation, are paradigms for the study of critical phenomena in statistical mechanics. Although these models have been studied by mathematicians for about half a century, exciting new developments continue to occur and the subject is flourishing. Much progress has been made, but it remains a major challenge for mathematical physics and probability theory to obtain a complete and mathematically rigorous understanding of the scaling theory of these models at criticality.

These lecture notes concern the lace expansion, which is a powerful tool for the analysis of the critical scaling of several models above their upper critical dimensions, namely:

- the self-avoiding walk on $\mathbb{Z}^{d}$ for $d>4$,

- lattice trees and lattice animals on $\mathbb{Z}^{d}$ for $d>8$,

- percolation on $\mathbb{Z}^{d}$ for $d>6$,

- oriented percolation on $\mathbb{Z}^{d} \times \mathbb{Z}_{+}$and the contact process on $\mathbb{Z}^{d}$ for $d>4$.

Results include proofs of existence of critical exponents, with mean-field values, and construction of scaling limits. Often, the scaling limit is described in terms of super-Brownian motion.

There are two distinct goals for these notes. The first goal is to provide a written accompaniment to my lectures at the Saint-Flour summer school in 2004, and at the Pacific Institute for the Mathematical Sciences - University of British Columbia summer school in 2005. The notes contain an introduction to the lace expansion and several of its applications, with sufficient background and depth to prepare a newcomer to do research using the lace expansion. Basic graduate level probability theory will be used, but no previous knowledge of the lace expansion or super-Brownian motion is assumed. The second goal is to provide a survey of the field, so that an interested reader can follow up by consulting the original literature. In pursuit of the second goal, these notes include more material than can be covered during a summer school course.

Following a brief initial section concerning random walk, the notes can be divided into four parts, whose contents are summarized as follows.

Part I, which concerns the self-avoiding walk, consists of Sections 2-6. A complete and self-contained proof is given of the convergence of the lace expansion for the nearest-neighbour model in dimensions $d \gg 4$, and for the spread-out model of self-avoiding walks which take steps of length at most $L$, with $L \gg 1$, in dimensions $d>4$. The convergence proof presented here seems simpler than all previous lace expansion convergence proofs. As a consequence of convergence, it is shown that the critical exponent $\gamma$ for the generating function of the number of $n$-step self-avoiding walks exists and is equal to 1 . A survey is then given of the many extensions of this result that have been obtained using the lace expansion. 
Part II, which concerns lattice trees and lattice animals, consists of Sections 7-8. It is shown how a minor modification of the expansion for the self-avoiding walk can be applied to give expansions for lattice trees and lattice animals, and an indication is given of the diagrammatic estimates that are necessary for proving convergence of the expansion. The relevance of the square condition is indicated, and results concerning existence of critical exponents in dimensions $d>8$ are surveyed.

Part III, which concerns percolation, oriented percolation, and the contact process, consists of Sections 9-14. Detailed discussions are given of expansions for each of these models. Differential inequalities involving the triangle condition are stated (and usually proved) and are shown to imply mean-field behaviour of various critical exponents. Results concerning existence of critical exponents in dimensions $d>6$ (for percolation) and $d>4$ (for oriented percolation and the contact process) are surveyed.

Part IV, which concerns super-Brownian scaling limits, consists of Sections 15-17. Critical branching random walk with Poisson offspring distribution is analyzed in detail and used to give a self-contained construction of integrated super-Brownian excursion (ISE). The role of ISE as the scaling limit of lattice trees and of critical percolation clusters, above the upper critical dimensions, is discussed. The canonical measure of super-Brownian motion is also described, as is its role as scaling limit of critical oriented percolation clusters and the critical contact process in dimensions $d>4$, and of lattice trees in dimensions $d>8$.

Mathematics is not a spectator sport, and true understanding requires active participation in working out the ideas. To help facilitate this, a number of exercises for the reader appear throughout the notes. Some can be solved in a few lines, and others require more effort. I am grateful to Jeremy Flowers, Jesse Goodman, Jeffrey Hood, Sandra Kliem, Richard Liang, and Terry Soo, who collectively wrote solutions to all the exercises during the PIMS-UBC summer school.

It would not be possible to include detailed proofs of all the results discussed in these lecture notes without substantially increasing their length, and a number of important topics are only alluded to. These include: the inductive approach to the lace expansion, which is in many respects the most powerful method to prove convergence of the expansion; the "double" expansions that have been used to analyze $r$-point functions for $r \geq 3$; and the lace expansion on a tree, which is a method that can sometimes be used to replace a double expansion. (Two of these topics - the inductive method and double expansions - are discussed in recent lecture notes by Remco van der Hofstad [110].) Also, a complete proof of the convergence of the expansion is given only for the self-avoiding walk. This is the simplest setting for proving convergence, and convergence for the other models can be based on the ideas used in this setting. Finally, in an important new development about which it is too early to provide details, Sakai [181] has shown how to apply the lace expansion to analyze the Ising model in dimensions $d>4$. 
This work was supported in part by NSERC of Canada. Versions of the lectures were given at the University of British Columbia in Spring 2003, at EURANDOM in Fall 2003, at Saint-Flour in Summer 2004, and at PIMS/UBC in Summer 2005. The lecture notes were written primarily while I was travelling during 2003-04. I thank EURANDOM and the Thomas Stieltjes Institute, the University of Melbourne, Microsoft Research, and my hosts at these institutions, for their hospitality during visits to Eindhoven, Melbourne and Redmond.

I am grateful to the friends and colleagues with whom I have had the good fortune to work on topics related to these lecture notes. I thank Markus Heydenreich, Remco van der Hofstad, Mark Holmes, Sandra Kliem, Ed Perkins and Akira Sakai for suggesting improvements and for comments on earlier drafts of these notes. Many others have also made helpful comments of one form or another. Most of the illustrations (and all of the best ones) were produced by Bill Casselman, my colleague at the University of British Columbia and Graphics Editor of Notices of the American Mathematical Society.

I extend special thanks to David Brydges, whose patient teaching brought me into the subject, and to Takashi Hara and Remco van der Hofstad, who have played profound roles in the development of the ideas presented in these notes.

Vancouver, August 9, 2005. 



\section{Contents}

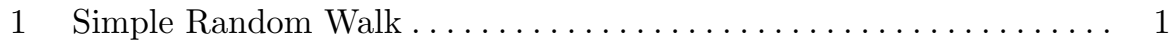

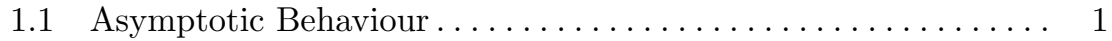

1.2 Universality and Spread-Out Models .................. 6

2 The Self-Avoiding Walk ......................... 6

2.1 Asymptotic Behaviour . . . . . . . . . . . . . . . . . 7

2.2 Differential Inequalities and the Bubble Condition . . . . . . . . 13

3 The Lace Expansion for the Self-Avoiding Walk . . . . . . . . . . . 17

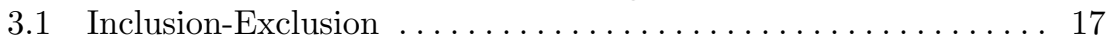

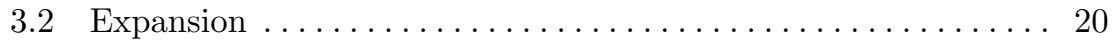

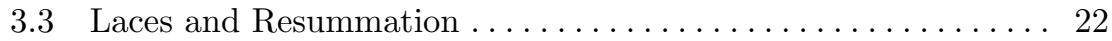

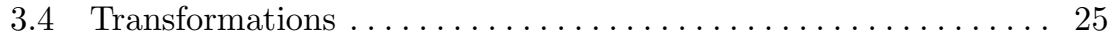

4 Diagrammatic Estimates for the Self-Avoiding Walk . . . . . . . . . 27

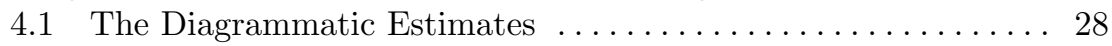

4.2 Proof of the Diagrammatic Estimates . . . . . . . . . . . . . . . . 29

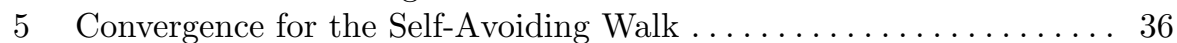

5.1 Random-Walk Estimates ..................... 37

5.2 Convergence of the Expansion .................. 41

5.3 Finite Bubble vs Small Bubble ................... 48

5.4 Differential Equality and the Bubble Condition ... . . . . . . . . 49

6 Further Results for the Self-Avoiding Walk . . . . . . . . . . . . . . 50

6.1 The Self-Avoiding Walk in Dimensions $d>4 \ldots \ldots \ldots \ldots \ldots$

6.2 A Self-Avoiding Walk in Dimension $d=1 \ldots \ldots \ldots \ldots \ldots \ldots$

6.3 Nearest-Neighbour Attraction . . . . . . . . . . . . . . . 55

6.4 Networks of Self-Avoiding Walks . . . . . . . . . . . . . 57

$7 \quad$ Lattice Trees . . . . . . . . . . . . . . . . . . . . . . . . . . 59

7.1 Asymptotic Behaviour. . . . . . . . . . . . . . . . . . 59

7.2 Differential Inequalities and the Square Condition . . . . . . . . . . 64

8 The Lace Expansion for Lattice Trees . . . . . . . . . . . . . . . . . . 68

8.1 Expansion for Lattice Trees . . . . . . . . . . . . . . 68

8.2 Expansion for Lattice Animals . . . . . . . . . . . . . . . 72

8.3 Diagrammatic Estimates for Lattice Trees . . . . . . . . . . . 74 


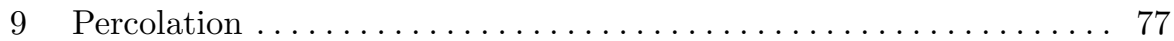

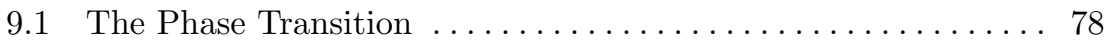

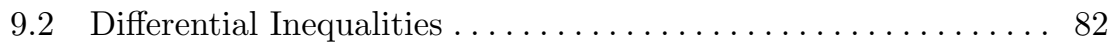

9.3 Differential Inequalities and the Triangle Condition ........ 85

9.4 Proofs of the Differential Inequalities . . . . . . . . . . . . 89

10 The Expansion for Percolation $\ldots \ldots \ldots \ldots \ldots \ldots \ldots \ldots \ldots \ldots$

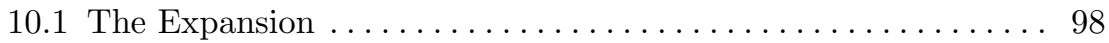

10.2 The Diagrams ................................. 105

10.3 Diagrammatic Estimates for Percolation ................ 111

11 Results for Percolation ................................... 113

11.1 Critical Exponents . . . . . . . . . . . . . . . . . . . 113

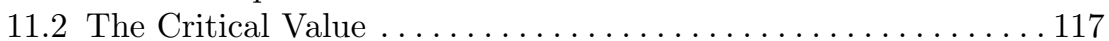

11.3 The Incipient Infinite Cluster . . . . . . . . . . . . . . . . . 118

11.4 Percolation on Finite Graphs . . . . . . . . . . . . . . . . . . . . 121

12 Oriented Percolation .................................... 127

12.1 The Phase Transition ......................... 127

12.2 The Infrared Bound and the Triangle Condition .......... 129

12.3 The Critical Value ............................. 133

12.4 The Incipient Infinite Cluster . . . . . . . . . . . . . . . . 133

13 Expansions for Oriented Percolation $\ldots \ldots \ldots \ldots \ldots \ldots \ldots \ldots \ldots \ldots \ldots$

13.1 Inclusion-Exclusion . . . . . . . . . . . . . . . . . . . 137

13.2 Laces and Resummation ........................ 139

13.3 Application of the Percolation Expansion ............... 141

13.4 The Upper Critical Dimension ...................... 142

13.5 Diagrams for Oriented Percolation . . . . . . . . . . . . . . . 143

14 The Contact Process . . . . . . . . . . . . . . . . . . . . . . . . . . . 143

14.1 The Phase Transition ............................ 144

14.2 Approximation by Oriented Percolation................ 146

14.3 The Infrared Bound and the Triangle Condition ........... . 146

14.4 Expansion for the Contact Process . . . . . . . . . . . . . 150

14.5 Diagrams for the Contact Process . . . . . . . . . . . . . . . 151

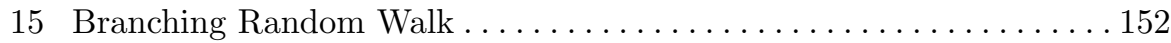

15.1 A Mean-Field Model .............................. 153

15.2 Branching Random Walk .......................... 154

15.3 Weakly Self-Avoiding Lattice Trees $\ldots \ldots \ldots \ldots \ldots \ldots \ldots \ldots 1$

16 Integrated Super-Brownian Excursion .................... 163

16.1 Moment Measures of Branching Random Walk ............ 164

16.2 Critical Exponents and Generating Functions ............. 165

16.3 Construction of ISE . . . . . . . . . . . . . . . . . . . . . . 169

16.4 Lattice trees and ISE . . . . . . . . . . . . . . . . . 174

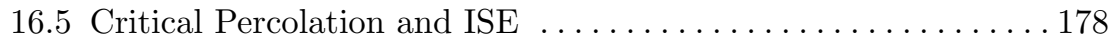

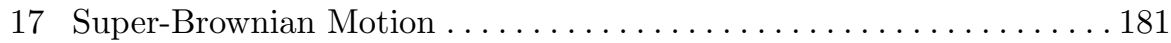

17.1 The Canonical Measure of SBM .................. 181

17.2 Moment Measures of the Canonical Measure ............. 184

17.3 Critical Oriented Percolation and SBM ................ 187 
17.4 The Critical Contact Process and SBM . . . . . . . . . . . . . . 189

17.5 Lattice Trees and SBM . . . . . . . . . . . . . . . . . . . . . . . 190

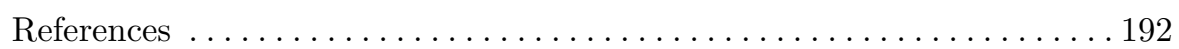


The Lace Expansion and its Applications 


\section{Simple Random Walk}

The point of departure for the lace expansion is simple (ordinary) random walk, and it is helpful first to recall some elementary facts about random walk on $\mathbb{Z}^{d}$. This will also set some notation for later use.

\subsection{Asymptotic Behaviour}

Fix a finite set $\Omega \subset \mathbb{Z}^{d}$ that is invariant under the symmetry group of $\mathbb{Z}^{d}$, i.e., under permutation of coordinates or replacement of any coordinate $x_{i}$ by $-x_{i}$. Our two basic examples are the nearest-neighbour model

$$
\Omega=\left\{x \in \mathbb{Z}^{d}:\|x\|_{1}=1\right\}
$$

and the spread-out model

$$
\Omega=\left\{x \in \mathbb{Z}^{d}: 0<\|x\|_{\infty} \leq L\right\},
$$

where $L$ is a fixed (usually large) constant. The norms are defined, for $x=$ $\left(x_{1}, \ldots, x_{d}\right)$, by $\|x\|_{1}=\sum_{j=1}^{d}\left|x_{j}\right|$ and $\|x\|_{\infty}=\max _{1 \leq j \leq d}\left|x_{j}\right|$.

For $n \geq 1$, an $n$-step walk taking steps in $\Omega$ is defined to be a sequence $(\omega(0), \omega(1), \ldots, \omega(n))$ of vertices in $\mathbb{Z}^{d}$ such that $\omega(i)-\omega(i-1) \in \Omega$ for $i=$ $1, \ldots, n$. Let $\mathcal{W}_{n}(x, y)$ be the set of $n$-step walks with $\omega(0)=x$ and $\omega(n)=y$, and let $\mathcal{W}_{n}=\cup_{x \in \mathbb{Z}^{d}} \mathcal{W}_{n}(0, x)$ denote the set of all $n$-step walks starting from the origin. Let $c_{n}^{(0)}(x)$ denote the cardinality of $\mathcal{W}_{n}(0, x)$. The superscript $(0)$ is there to indicate that we are working with the random walk with no interaction. We allow for the degenerate case $n=0$ by defining $\mathcal{W}_{0}(x, y)$ to consist of the zero-step walk $(x)$ if $x=y$, and to be empty otherwise. Then $c_{0}^{(0)}(x, y)=\delta_{x, y}$. Taking into account the translation invariance, we will use the abbreviations $\mathcal{W}_{n}(y-x)=\mathcal{W}_{n}(x, y)$ and $c_{n}^{(0)}(y-x)=c_{n}^{(0)}(x, y)$.

For $n \geq 1$, by considering the possible values $y \in \Omega$ of the walk's first step, we have

$$
c_{n}^{(0)}(x)=\sum_{y \in \Omega} c_{n-1}^{(0)}(x-y)=\sum_{y \in \mathbb{Z}^{d}} c_{1}^{(0)}(y) c_{n-1}^{(0)}(x-y) .
$$

Denoting the convolution of functions $f$ and $g$ by

$$
(f * g)(x)=\sum_{y \in \mathbb{Z}^{d}} f(y) g(x-y),
$$

(1.3) can be written as

$$
c_{n}^{(0)}(x)=\left(c_{1}^{(0)} * c_{n-1}^{(0)}\right)(x) .
$$

The Fourier transform of an absolutely summable function $f: \mathbb{Z}^{d} \rightarrow \mathbb{C}$ is defined by 


$$
\hat{f}(k)=\sum_{x \in \mathbb{Z}^{d}} f(x) \mathrm{e}^{\mathrm{i} k \cdot x} \quad\left(k \in[-\pi, \pi]^{d}\right),
$$

where $k \cdot x=\sum_{j=1}^{d} k_{j} x_{j}$, with inverse

$$
f(x)=\int_{[-\pi, \pi]^{d}} \frac{\mathrm{d}^{d} k}{(2 \pi)^{d}} \hat{f}(k) \mathrm{e}^{-\mathrm{i} k \cdot x} .
$$

The fact stated in part (a) of the following exercise makes the use of Fourier transforms very convenient.

Exercise 1.1. (a) Show that the Fourier transform of $f * g$ is $\hat{f} \hat{g}$.

(b) A closely related statement is the following. Denote the generating functions of the sequences $f_{n}$ and $g_{n}$ by $F(z)=\sum_{n=0}^{\infty} f_{n} z^{n}$ and $G(z)=$ $\sum_{n=0}^{\infty} g_{n} z^{n}$, and assume these series both have positive radius of convergence. Show that the generating function $H(z)$ of the sequence $h_{n}=\sum_{m=0}^{n} f_{m} g_{n-m}$ is $H(z)=F(z) G(z)$.

By Exercise 1.1(a), (1.5) implies that

$$
\hat{c}_{n}^{(0)}(k)=\hat{c}_{1}^{(0)}(k) \hat{c}_{n-1}^{(0)}(k) .
$$

Since $\hat{c}_{0}^{(0)}(k)=1$, solving $(1.8)$ by iteration gives

$$
\hat{c}_{n}^{(0)}(k)=\hat{c}_{1}^{(0)}(k)^{n} \quad(n \geq 0) .
$$

If we define the transition probability

$$
D(x)=\frac{1}{|\Omega|} I[x \in \Omega]=\frac{1}{|\Omega|} c_{1}^{(0)}(x),
$$

where $|\Omega|$ denotes the cardinality of the set $\Omega$ and $I$ denotes the indicator function, then (1.9) can be rewritten as

$$
\hat{c}_{n}^{(0)}(k)=|\Omega|^{n} \hat{D}(k)^{n} \quad(n \geq 0) .
$$

Exercise 1.2. (a) Show that for the nearest-neighbour model,

$$
\hat{D}(k)=\frac{1}{d} \sum_{j=1}^{d} \cos k_{j},
$$

and for the spread-out model

$$
\hat{D}(k)=\frac{1}{|\Omega|}\left[\prod_{j=1}^{d} M\left(k_{j}\right)-1\right],
$$

where 


$$
M(t)=\frac{\sin [(2 L+1) t / 2]}{\sin (t / 2)}
$$

is the Dirichlet kernel.

(b) Denote the variance of $D$ by $\sigma^{2}=\sum_{x \in \mathbb{Z}^{d}}|x|^{2} D(x)$. Show that $\sigma=1$ for the nearest-neighbour model and that $\sigma$ is asymptotic to a multiple of $L$ as $L \rightarrow \infty$ for the spread-out model.

The number of $n$-step walks starting from a given vertex is of course $|\Omega|^{n}$, because each step can be chosen in $|\Omega|$ different ways. This fact is contained in (1.11), since the number of $n$-step walks starting from the origin is $\sum_{x \in \mathbb{Z}^{d}} c_{n}^{(0)}(x)=\hat{c}_{n}^{(0)}(0)=|\Omega|^{n}$, using $\hat{D}(0)=1$.

By symmetry, $\sigma^{2}=-\left.\nabla^{2} \hat{D}\right|_{k=0}$, where $\nabla^{2}=\sum_{j=1}^{d} \nabla_{j}^{2}$ is the Laplacian, with $\nabla_{j}$ denoting partial differentiation with respect to the component $k_{j}$ of $k$. Then, by (1.11) and by the symmetry of $\Omega$, the central limit theorem

$$
\lim _{n \rightarrow \infty} \frac{\hat{c}_{n}^{(0)}(k / \sigma \sqrt{n})}{\hat{c}_{n}^{(0)}(0)}=\mathrm{e}^{-|k|^{2} / 2 d}
$$

follows, as does the fact that the mean-square displacement is given by

$$
\frac{\sum_{x \in \mathbb{Z}^{d}}|x|^{2} c_{n}^{(0)}(x)}{\sum_{x \in \mathbb{Z}^{d}} c_{n}^{(0)}(x)}=-\left.\nabla^{2} \hat{D}^{n}\right|_{k=0}=n \sigma^{2} .
$$

Exercise 1.3. Prove (1.15) and (1.16).

The two-point function is defined by

$$
C_{z}(x, y)=\sum_{n=0}^{\infty} \sum_{\omega \in \mathcal{W}_{n}(x, y)} z^{n}=\sum_{n=0}^{\infty} c_{n}^{(0)}(x, y) z^{n}
$$

The two-point function is finite for $z \in[0,1 /|\Omega|)$. For $d>2$, it is also known to be finite for $z=1 /|\Omega|$, and for this value of $z$ it is called the Green function. By translation invariance, we may regard the two-point function as a function of a single variable, writing $C_{z}(x, y)=C_{z}(y-x)$. By (1.11) and (1.17), its Fourier transform is

$$
\hat{C}_{z}(k)=\sum_{n=0}^{\infty} \hat{c}_{n}^{(0)}(k) z^{n}=\frac{1}{1-z|\Omega| \hat{D}(k)} .
$$

The susceptibility is defined by

$$
\chi(z)=\sum_{x \in \mathbb{Z}^{d}} C_{z}(0, x)=\hat{C}_{z}(0)=\frac{1}{1-z|\Omega|} .
$$

The critical point is the singularity $z_{c}=1 /|\Omega|$ of the susceptibility. 
The inverse Fourier transform of (1.18) is

$$
C_{z}(x)=\int_{[-\pi, \pi]^{d}} \frac{\mathrm{d}^{d} k}{(2 \pi)^{d}} \frac{\mathrm{e}^{-\mathrm{i} k \cdot x}}{1-z|\Omega| \hat{D}(k)} .
$$

For $d>2$,

$$
C_{z_{c}}(x) \sim \operatorname{const} \frac{1}{|x|^{d-2}}
$$

as $|x| \rightarrow \infty$, where the constant depends on $d$ and on $\Omega$ (see $[149,195]$, or [203] for a more general statement of this fact). The notation

$$
f(x) \sim g(x) \quad \text { denotes } \quad \lim _{x \rightarrow \infty} f(x) / g(x)=1
$$

and this notation will used in general for asymptotic formulas.

Exercise 1.4. Some care is needed with (1.20) when $z=z_{c}$, since $C_{z_{c}}(x)$ is not summable by (1.21) and thus its Fourier transform is problematic. Using the symmetry of $\Omega$, prove that (1.20) does hold when $z=z_{c}$ for $d>2$, and that the integral is infinite when $z=z_{c}$ for $d \leq 2$.

Exercise 1.5. Let $f: \mathbb{Z}^{d} \rightarrow \mathbb{C}$. For $y \in \Omega$, define forward and backward discrete partial derivatives by $\partial_{y}^{+} f(x)=f(x+y)-f(x)$ and $\partial_{y}^{-} f(x)=f(x)-$ $f(x-y)$. Define the discrete Laplacian by

$$
\Delta f(x)=\frac{1}{2} \frac{1}{|\Omega|} \sum_{y \in \Omega} \partial_{y}^{-} \partial_{y}^{+} f(x)=\frac{1}{|\Omega|} \sum_{y \in \Omega} f(x+y)-f(x),
$$

and let $\delta_{x, y}$ denote the Kronecker delta which takes the value 1 if $x=y$ and 0 if $x \neq y$. Show that $-\Delta C_{1 /|\Omega|}(x)=\delta_{0, x}$. Thus $C_{1 /|\Omega|}(x)$ is the Green function for $-\Delta$.

Exercise 1.6. Consider a simple random walk started at the origin.

(a) Let $u$ denote the probability that the walk ever returns to the origin. The walk is recurrent if $u=1$ and transient if $u<1$. Let $N$ denote the (random) number of visits to the origin, including the initial visit at time 0 , and let $m=\mathbb{E} N$. Show that $m=\frac{1}{1-u}$, so the walk is recurrent if and only if $m=\infty$. (b) Show that

$$
m=\sum_{n=0}^{\infty} \mathbb{P}(\omega(n)=0)=\int_{[-\pi, \pi]^{d}} \frac{1}{1-\hat{D}(k)} \frac{\mathrm{d}^{d} k}{(2 \pi)^{d}}
$$

Thus transience is characterized by the integrability of $\hat{C}_{1 /|\Omega|}(k)$.

(c) For simplicity, consider the nearest-neighbour model, with $\Omega$ given by (1.1). Show that the walk is recurrent in dimensions $d \leq 2$ and transient in dimensions $d>2$. 
Exercise 1.7. Let $\omega^{(1)}$ and $\omega^{(2)}$ denote two independent simple random walks started at the origin, and let

$$
X=\sum_{i=0}^{\infty} \sum_{j=0}^{\infty} I\left[\omega^{(1)}(i)=\omega^{(2)}(j)\right]
$$

denote the number of intersections of the two walks. Here $I$ denotes an indicator function. Show that

$$
\mathbb{E} X=\int_{[-\pi, \pi]} \frac{1}{[1-\hat{D}(k)]^{2}} \frac{\mathrm{d}^{d} k}{(2 \pi)^{d}} .
$$

Thus $\mathbb{E} X$ is finite if and only if $\hat{C}_{1 /|\Omega|}(k)$ is square integrable. Conclude, for simplicity for the nearest-neighbour model, that the expected number of intersections is finite if $d>4$ and infinite if $d \leq 4$.

The integral $(2 \pi)^{-d} \int_{[-\pi, \pi]^{d}} \hat{C}_{z_{c}}(k)^{2} \mathrm{~d}^{d} k$ of (1.26) is equal, by the Parseval relation, to $\sum_{x \in \mathbb{Z}^{d}} C_{z_{c}}(x)^{2}$. The relevance of the condition $d>4$ for the latter is evident from the asymptotic behaviour (1.21). However, the $k$-space analysis is more elementary, as it relies on the easy formulas given in (1.12) and (1.18) rather than the deeper statement (1.21). It is often much easier to use estimates in $k$-space than to work directly in $x$-space.
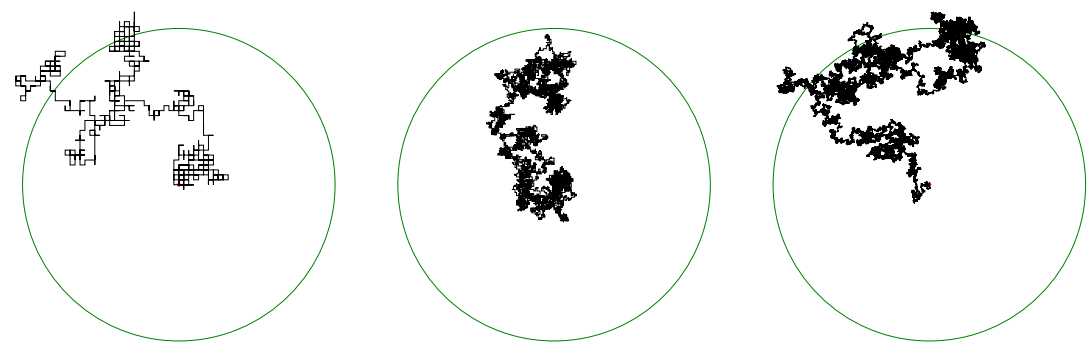

Fig. 1.1. Nearest-neighbour random walks on $\mathbb{Z}^{2}$ taking $n=1,000,10,000$ and 100,000 steps. The circles have radius $\sqrt{n}$, in units of the step size of the random walk.

It is a consequence of Donsker's Theorem [24] that the scaling limit of simple random walk is Brownian motion, in all dimensions. This means that if we define a random continuous function $X_{n}$ from the interval $[0,1]$ into $\mathbb{R}^{d}$ by setting $X_{n}(j / n)=\sigma^{-1} n^{-1 / 2} \omega(j)$ for integers $j \in[0, n]$, and interpolating linearly between consecutive vertices, then the distribution of $X_{n}$ converges weakly to the Wiener measure. See Fig. 1.1. 


\subsection{Universality and Spread-Out Models}

In these notes, we study several models that live on the integer lattice, and each has a nearest-neighbour and a spread-out version. In the nearestneighbour model, specified by (1.1), bonds (also called edges) join pairs of vertices separated by unit Euclidean distance. In the spread-out model, specified by (1.2), bonds join pairs of vertices separated by distance between 1 and $L$, where $L$ is a parameter usually taken to be large. According to the deep hypothesis of universality, the critical scaling of the models to be studied should be the same for the nearest-neighbour and spread-out models.

We use the spread-out model because proofs of convergence of the lace expansion require large degree. The degree is the cardinality of $\Omega$. For the nearest-neighbour model the degree is $2 d$, and can be taken large by increasing the dimension. The degree of the spread-out model is of order $L^{d}$ for large $L$, and this allows for convergence proofs for the lace expansion without taking the dimension $d$ to be large in an uncontrolled way. In the applications to be discussed, results will typically be obtained: (i) for the nearest-neighbour model for $d \geq d_{0}$ for some $d_{0}$ having no physical meaning, and (ii) for the spread-out model with $L$ larger than some $L_{0}$ and $d$ strictly greater than the upper critical dimension (4 for the self-avoiding walk, oriented percolation and the contact process; 6 for percolation; 8 for lattice trees and lattice animals). While it is of interest to prove results of type (i) with $d_{0}$ equal to the upper critical dimension plus one, failing this, results of type (ii) seem more important, as they indicate clearly the role of the upper critical dimension. Also, the fact that all large $L$ give rise to the same scaling behaviour provides a partial proof of universality in this context. In fact, much more general spread-out models than (1.2) can be handled using the lace expansion (see, e.g., $[94,120])$, but we restrict attention in these notes to (1.2) for the sake of simplicity.

\section{The Self-Avoiding Walk}

The self-avoiding walk is a model of fundamental interest in combinatorics, probability theory, statistical physics and polymer chemistry. It is a model of random walk paths but it cannot be described in terms of transition probabilities and thus is not even a stochastic process. It is certainly non-Markovian. These features makes the subject difficult, and many of the central problems remain unsolved. See [127, 158] for extensive surveys.

The self-avoiding walk is a basic example in the theory of critical phenomena, due to its close links with models of ferromagnetism such as the Ising model. In particular, it can be understood as the $N \rightarrow 0$ limit of the $N$-vector model [79] (see also [158, Section 2.3]). In polymer chemistry, self-avoiding walks are used to model a single linear polymer molecule in a good solution 
$[80,205]$. The flexibility of the polymer is modelled by the possible configurations of a self-avoiding walk, while the self-avoidance constraint models the excluded volume effect that causes the polymer to repel itself.

In this section, we first give an overview of the self-avoiding walk and its predicted asymptotic behaviour. Then we define the bubble condition and show that it is a sufficient condition for a particular critical exponent (namely $\gamma$ ) to exist and take its mean-field value.

\subsection{Asymptotic Behaviour}

An $n$-step self-avoiding walk starting at $x$ and ending at $y$ is an $n$-step walk $(\omega(0), \omega(1), \ldots, \omega(n))$ with $\omega(0)=x, \omega(n)=y$, and $\omega(i) \neq \omega(j)$ for all $i \neq j$. We will assume for simplicity that the walks take steps in $\Omega$ given either by (1.1) or (1.2). Let $\mathcal{S}_{n}(x, y)$ be the set of $n$-step self-avoiding walks from $x$ to $y$, let $\mathcal{S}_{n}=\cup_{x \in \mathbb{Z}^{d}} \mathcal{S}_{n}(0, x)$ denote the set of all $n$-step self-avoiding walks starting from the origin, and let $\mathcal{S}(x, y)=\cup_{n=0}^{\infty} \mathcal{S}_{n}(x, y)$ denote the set of all self-avoiding walks of any length from $x$ to $y$. Let $c_{n}(x, y)$ denote the cardinality of $\mathcal{S}_{n}(x, y)$. In particular, $c_{0}(x, y)=\delta_{x, y}$. We will use the abbreviations $\mathcal{S}_{n}(x)=\mathcal{S}_{n}(0, x), c_{n}(x)=c_{n}(0, x)$, and $c_{n}=\sum_{x \in \mathbb{Z}^{d}} c_{n}(x)$. Thus $c_{n}$ counts the number of $n$-step self-avoiding walks that start at the origin and end anywhere.

More generally, given a walk $\omega$, let

$$
U_{s t}(\omega)=\left\{\begin{aligned}
&-1 \text { if } \omega(s)=\omega(t) \\
& 0 \text { if } \omega(s) \neq \omega(t)
\end{aligned}\right.
$$

and, for $\lambda \in[0,1]$, let

$$
c_{n}^{(\lambda)}(x)=\sum_{\omega \in \mathcal{W}_{n}(x)} \prod_{0 \leq s<t \leq n}\left(1+\lambda U_{s t}(\omega)\right) .
$$

For $\lambda=0,(2.2)$ is the same as the quantity $c_{n}^{(0)}(x)$ defined previously. For $\lambda=1$, we have $c_{n}^{(1)}(x)=c_{n}(x)$, and we will usually omit the superscript ${ }^{(1)}$ when $\lambda=1$. For $0<\lambda<1$, (2.2) defines a much-studied model of weakly self-avoiding walks (sometimes called the Domb-Joyce model after [64]) in which walks with self intersections receive less weight than walks that are self-avoiding.

For $\lambda \in[0,1]$, let

$$
c_{n}^{(\lambda)}=\sum_{x \in \mathbb{Z}^{d}} c_{n}^{(\lambda)}(x) .
$$

Since $1+\lambda U_{s t}(\omega) \leq 1$ for all $s, t, \omega$, we have

$$
\prod_{0 \leq s<t \leq m+n}\left(1+\lambda U_{s t}(\omega)\right) \leq \prod_{0 \leq s<t \leq m}\left(1+\lambda U_{s t}(\omega)\right) \prod_{m \leq s<t \leq m+n}\left(1+\lambda U_{s t}(\omega)\right),
$$


from which we easily conclude that

$$
c_{m+n}^{(\lambda)} \leq c_{m}^{(\lambda)} c_{n}^{(\lambda)} .
$$

Therefore, $\log c_{n}^{(\lambda)}$ is a subadditive sequence. By a standard lemma [158, Lemma 1.2.2], it follows that the limit

$$
\mu_{\lambda}=\lim _{n \rightarrow \infty}\left(c_{n}^{(\lambda)}\right)^{1 / n}
$$

exists and that moreover

$$
c_{n}^{(\lambda)} \geq \mu_{\lambda}^{n} .
$$

When $\lambda=1, \mu=\mu_{1}$ is known as the connective constant. For nearestneighbour walks, it is easy to see that $d \leq \mu \leq 2 d-1$. The lower bound follows from the fact that $c_{n}$ is at least as large as the number $d^{n}$ of walks that take steps only in the positive coordinate directions. The upper bound follows from the fact that $c_{n}$ is at most the number $2 d(2 d-1)^{n-1}$ of walks that never reverse a previous step. The exact value of $\mu$ is not known in general, although good rigorous numerical upper and lower bounds have been obtained $[55,106,171,187]$. Numerical estimates of $\mu$ are 2.638 and 4.684 for nearestneighbour self-avoiding walks in dimensions 2 and 3 respectively — in fact there are higher precision estimates due to A.J. Guttmann and coworkers. It has been conjectured [168, 169, 170], and been confirmed by numerical evidence [70], that on the 2-dimensional hexagonal lattice $\mu=\sqrt{2+\sqrt{2}}$. It has been observed from enumeration data that on the 2-dimensional square lattice $\mu$ is very well approximated by the reciprocal of the smallest positive root of the quartic equation $581 x^{4}+7 x^{2}-13=0$ [54,137], although no derivation or explanation of this equation has been discovered.

For the nearest-neighbour self-avoiding walk on $\mathbb{Z}^{d}$, the lace expansion has been used to prove that $\mu(d)$ has an asymptotic expansion to all orders in $1 / d$, with integer coefficients, and that

$$
\begin{aligned}
\mu(d)= & 2 d-1-\frac{1}{2 d}-\frac{3}{(2 d)^{2}}-\frac{16}{(2 d)^{3}}-\frac{102}{(2 d)^{4}}-\frac{729}{(2 d)^{5}} \\
& -\frac{5533}{(2 d)^{6}}-\frac{42229}{(2 d)^{7}}-\frac{288761}{(2 d)^{8}}+O\left(\frac{1}{(2 d)^{9}}\right) .
\end{aligned}
$$

Without using the lace expansion (which was not yet invented), the above coefficients were computed in [74], up to and including $-102(2 d)^{-4}$, without a rigorous estimate for the error. About the same time, the formula $\mu(d)=$ $2 d-1-(2 d)^{-1}+O\left((2 d)^{-2}\right)$ was proved in [140]. In [101, 102], the lace expansion was used to prove the existence of an asymptotic expansion to all orders, and also that $\mu(d)=2 d-1-(2 d)^{-1}-3(2 d)^{-2}-16(2 d)^{-3}-102(2 d)^{-4}+O\left((2 d)^{-5}\right)$. The four additional coefficients in (2.8) were obtained in [53]. It seems likely that the asymptotic expansion has radius of convergence zero, though there is no proof of this. For further $1 / d$ expansion results (but without rigorous 
error estimates) see $[77,163,164]$. For asymptotics of the connective constant for the spread-out model, as $L \rightarrow \infty$, see $[118,176]$.

For $\lambda=0$, we have seen in Section 1 that $c_{n}^{(0)}=|\Omega|^{n}$, and thus the number of $n$-step walks grows purely exponentially in $n$. There is overwhelming evidence to support the belief that for $\lambda \in(0,1]$, the asymptotic form of $c_{n}^{(\lambda)}$ is given by

$$
c_{n}^{(\lambda)} \sim A_{\lambda} \mu_{\lambda}^{n} n^{\gamma-1} .
$$

Here, $A_{\lambda}$ is a constant which, like $\mu_{\lambda}$, depends on $\lambda, d$ and $\Omega$, but the critical exponent $\gamma$ is independent of $\lambda$ and $\Omega$ and is given by

$$
\gamma=\left\{\begin{array}{lr}
1 & \text { if } d=1 \\
\frac{43}{32} & \text { if } d=2 \\
1.162 \ldots & \text { if } d=3 \\
1 \text { with logarithmic corrections } & \text { if } d=4 \\
1 & \text { if } d \geq 5
\end{array}\right.
$$

The conjectured logarithmic correction in four dimensions, predicted by the renormalization group method, is

$$
c_{n}^{(\lambda)} \sim A_{\lambda} \mu_{\lambda}^{n}(\log n)^{1 / 4} \quad \text { if } d=4 .
$$

The independence of $\gamma$ on $\lambda \in(0,1]$ and $\Omega$ is referred to as universality. Similarly, the power of the logarithm in (2.11) is believed to be universal.

The exponent $\gamma$ has the following probabilistic interpretation. Consider the case $\lambda=1$, and let $q_{n}$ denote the probability that two independent $n$-step self-avoiding walks started at the origin do not have any intersection apart from their common starting point. Since a non-intersecting pair of $n$-step selfavoiding walks comprises a single $2 n$-step self-avoiding walk, if (2.9) holds then

$$
q_{n}=\frac{c_{2 n}}{c_{n}^{2}} \sim \frac{2^{\gamma-1}}{A_{1}} \frac{1}{n^{\gamma-1}} .
$$

In dimensions $d>4$, the lace expansion has been used to prove that (2.9) holds with $\gamma=1$ for various choices of $\lambda$ and $\Omega$, including the nearestneighbour model with $\lambda=1[96,97,98]$. Note that $\gamma=1$ corresponds to purely exponential growth on the right hand side of (2.9), as is the case for the simple random walk. Also, there is no decay as $n \rightarrow \infty$ in (2.12) when $\gamma=1$. Partial results for the 4-dimensional case have been obtained in [43, 44, 129] (physics references include $[38,65])$. The 3 -dimensional case is completely unsolved mathematically. Evidence strongly supporting the value $\gamma=\frac{43}{32}$, which was first predicted in [168, 169, 170], has been obtained in [150], by associating the 2-dimensional self-avoiding walk with $\mathrm{SLE}_{8 / 3}$. Numerical tests supporting the role of $\mathrm{SLE}_{8 / 3}$ in the description of the 2-dimensional self-avoiding walk can be found in [139]. For $d=1$, the strictly self-avoiding nearest-neighbour model is trivial and $c_{n}^{(1)}=2$ for all $n \geq 1$, so $\gamma=1$. For the 1-dimensional strictly self-avoiding spread-out model, or for the weakly self-avoiding walk 
for any $\lambda \in(0,1)$, the determination of $c_{n}(\lambda)$ is no longer trivial, but has been analyzed in detail (see $[16,84,116,146])$.

For $d=2,3,4$, the best upper bounds on $c_{n}$ (with $\lambda=1$ ) are still the forty-year-old bounds

$$
\mu^{n} \leq c_{n} \leq \begin{cases}\mu^{n} \exp \left[K n^{1 / 2}\right] & \text { if } d=2 \\ \mu^{n} \exp \left[K n^{2 /(2+d)} \log n\right] & \text { if } d=3,4\end{cases}
$$

for a positive constant $K[88,140]$ (see also [158, Chapter 3]). This is a long way from (2.9).

We can define a measure on $\mathcal{W}_{n}$ by

$$
\mathbb{E}_{n}^{(\lambda)} X=\frac{1}{c_{n}^{(\lambda)}} \sum_{\omega \in \mathcal{W}_{n}} X(\omega) \prod_{0 \leq s<t \leq n}\left(1+\lambda U_{s t}(\omega)\right) .
$$

Exercise 2.1. For $\lambda=1$, the above measure is the uniform measure on $\mathcal{S}_{n}$. A family of probability measures $P_{m}$ on $\mathcal{S}_{n}$ is called consistent if $P_{m}(\omega)=$ $\sum_{\rho>\omega} P_{n}(\rho)$ for all $n \geq m$ and for all $\omega \in \mathcal{S}_{n}$, where the sum is over all $\rho$ whose first $m$ steps agree with $\omega$. Show that the uniform measure does not provide a consistent family.

The mean-square displacement is $\mathbb{E}_{n}^{(\lambda)}|\omega(n)|^{2}$ and it is believed that

$$
\mathbb{E}_{n}^{(\lambda)}|\omega(n)|^{2} \sim v_{\lambda} n^{2 \nu}
$$

where $v_{\lambda}$ is a constant depending on $\lambda, d, \Omega$, and where $\nu$ is universal and given by

$$
\nu= \begin{cases}1 & \text { if } d=1 \\ \frac{3}{4} & \text { if } d=2 \\ 0.588 \ldots & \text { if } d=3 \\ \frac{1}{2} \text { with logarithmic corrections } & \text { if } d=4 \\ \frac{1}{2} & \text { if } d \geq 5 .\end{cases}
$$

The conjectured logarithmic correction to $\nu$ in four dimensions, predicted by the renormalization group, is

$$
\mathbb{E}_{n}^{(\lambda)}|\omega(n)|^{2} \sim v_{\lambda} n(\log n)^{1 / 4} \quad \text { if } d=4 .
$$

In dimensions $d>4$, the lace expansion has been used to prove that (2.15) holds with $\nu=1 / 2$ for various choices of $\lambda$ and $\Omega$, including the nearestneighbour model with $\lambda=1$ [97, 98]. Partial results for $d=4$ have been obtained in $[43,44,129]$. For $d=2,3,4$, for the nearest-neighbour model with $\lambda=1$, it is still an open problem even to prove the "obvious" bounds that the mean-square displacement is bounded below by $n$ (cf. (1.16)) or bounded above by const $n^{2-\epsilon}$ for some $\epsilon>0$. For $d=1$, the ballistic behaviour $\nu=1$ is obvious for the strictly self-avoiding nearest-neighbour model. It is not obvious that $\nu=1$ for the 1-dimensional strictly self-avoiding spread-out model, or 

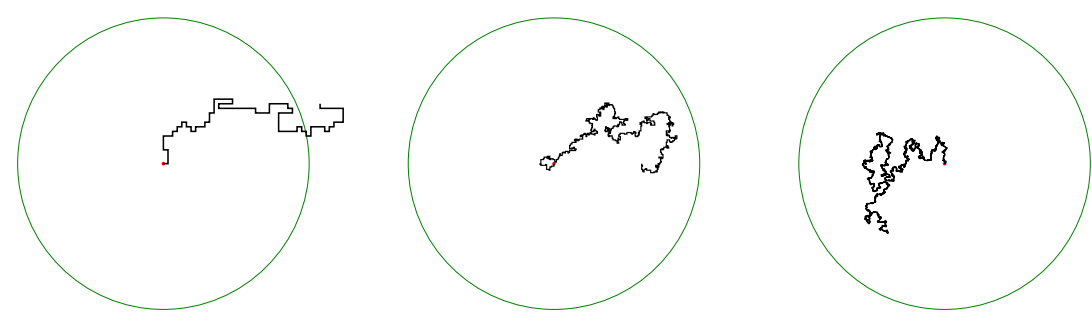

Fig. 2.1. Nearest-neighbour self-avoiding walks on $\mathbb{Z}^{2}$ taking $n=100,1,000$ and 10,000 steps, generated using the pivot algorithm [159]. The circles have radius $n^{3 / 4}$, in units of the step size of the self-avoiding walk.

for the 1-dimensional weakly self-avoiding walk, but ballistic behaviour has been proved also in these cases $[84,146]$.

The two-point function is defined by

$$
G_{z}^{(\lambda)}(x)=\sum_{n=0}^{\infty} c_{n}^{(\lambda)}(x) z^{n}
$$

and the susceptibility by

$$
\chi^{(\lambda)}(z)=\sum_{x \in \mathbb{Z}^{d}} G_{z}^{(\lambda)}(x)=\sum_{n=0}^{\infty} c_{n}^{(\lambda)} z^{n} .
$$

The latter has radius of convergence $z_{c}^{(\lambda)}=1 / \mu_{\lambda}$, by (2.6). For $\lambda=1$, a proof that the two-point function also has this radius of convergence is given in $[158$, Corollary 3.2.6].

Exercise 2.2. Show that the 1-dimensional strictly self-avoiding walk $(\lambda=1)$ two-point function is given by

$$
\hat{G}_{z}(k)=\frac{1-z^{2}}{1+z^{2}-2 z \cos k} .
$$

For $\lambda \in[0,1]$ and $z \in\left(0, z_{c}^{(\lambda)}\right)$, the two-point function decays exponentially. To see this for the nearest-neighbour model, we note that $c_{n}^{(\lambda)}(x)=0$ for $n<\|x\|_{\infty}$, and hence

$$
G_{z}^{(\lambda)}(x)=\sum_{n=\|x\|_{\infty}}^{\infty} c_{n}^{(\lambda)}(x) z^{n} \leq \sum_{n=\|x\|_{\infty}}^{\infty} c_{n}^{(\lambda)} z^{n} .
$$

Since $\left(c_{n}^{(\lambda)}\right)^{1 / n} \rightarrow \mu_{\lambda}$ by $(2.6)$, for any $\epsilon>0$ there is a positive $K_{\epsilon, \lambda}$ such that 


$$
c_{n}^{(\lambda)} \leq K_{\epsilon, \lambda}\left(\mu_{\lambda}+\epsilon\right)^{n}
$$

for all $n \geq 1$. Given a positive $z<z_{c}^{(\lambda)}=\mu_{\lambda}^{-1}$, we choose $\epsilon(z)>0$ such that $\theta_{z, \lambda}=\left(\mu_{\lambda}+\epsilon(z)\right) z<1$. Then substitution of (2.22) into (2.21) gives

$$
G_{z}^{(\lambda)}(x) \leq C_{z, \lambda} \exp \left[-\left|\log \theta_{z, \lambda}\right|\|x\|_{\infty}\right]
$$

with $C_{z, \lambda}=K_{\epsilon(z), \lambda}\left(1-\theta_{z, \lambda}\right)^{-1}$. This shows the desired exponential decay of the subcritical two-point function.

Precise asymptotics of the subcritical two-point function are known in detail. This has been primarily studied for the nearest-neighbour model with $\lambda=1$, and we assume this for the moment. First, it can be shown that for each $z \in\left(0, z_{c}\right)$ there is a norm $|\cdot|_{z}$ on $\mathbb{R}^{d}$ satisfying $\|u\|_{\infty} \leq|u|_{z} \leq\|u\|_{1}$ for every $u \in \mathbb{R}^{d}$, such that the limit

$$
m(z)=\lim _{|x|_{z} \rightarrow \infty} \frac{-\log G_{z}(x)}{|x|_{z}}
$$

exists and is finite [158, Theorem 4.1.18]. The correlation length is defined by

$$
\xi(z)=\frac{1}{m(z)} .
$$

Detailed asymptotics of the subcritical two-point function, known as OrnsteinZernike decay, were obtained in $[48,130]$. It is known that $\xi(z) \rightarrow \infty$ as $z \rightarrow z_{c}^{-}$ (see, e.g., [158, Corollary 4.1.15]), and it is predicted that

$$
\xi(z) \sim \operatorname{const} \frac{1}{\left(1-z / z_{c}\right)^{\nu}} \quad \text { as } z \rightarrow z_{c}^{-},
$$

with the same exponent $\nu$ as in (2.16).

For $\lambda \in(0,1]$, it is predicted that the exponential decay of the subcritical two-point function is replaced at $z=z_{c}$ by

$$
G_{z_{c}}^{(\lambda)}(x) \sim \operatorname{const} \frac{1}{|x|^{d-2+\eta}} \quad \text { as }|x| \rightarrow \infty
$$

and

$$
\hat{G}_{z_{c}}^{(\lambda)}(k) \sim \operatorname{const} \frac{1}{|k|^{2-\eta}} \quad \text { as } k \rightarrow 0
$$

with $\eta$ given in terms of $\gamma$ and $\nu$ by Fisher's relation $\gamma=(2-\eta) \nu$ (and with no logarithmic correction for $d=4$, to leading order). Equation (2.27) has been proved (with $\eta=0$ ) for the nearest-neighbour model in dimensions $d \geq 5$ [90], using the lace expansion. The $k$-space asymptotics are easier and are also known for the nearest-neighbour model when $d \geq 5$. Equation (2.27) has also been proved for the spread-out model with $d>4$ and $L$ sufficiently large [91]. In [39, 44], (2.27) is proved for a 4-dimensional hierarchical model with $\lambda$ sufficiently small (again with $\eta=0$ ). 
It is also believed that, for all $\lambda \in(0,1]$,

$$
\chi^{(\lambda)}(z) \sim \operatorname{const} \frac{1}{\left(1-z / z_{c}\right)^{\gamma}} \quad \text { as } z \rightarrow z_{c}^{-}
$$

with a multiplicative factor $\left[\log \left(1-z / z_{c}\right)\right]^{1 / 4}$ when $d=4$. This has been proved using the lace expansion for the nearest-neighbour model with $d>4$ and $\lambda=1$, with $\gamma=1[97,98]$. In Section 5.4 , we will see how to prove $(2.29)$, with $\gamma=1$, for the spread-out model with $d>4, \lambda=1$, and $L$ sufficiently large, and for the nearest-neighbour model with $\lambda=1$ and $d$ sufficiently large.

The scaling limit, assuming it exists, is the law of the path $n^{-\nu} \omega$ in the limit $n \rightarrow \infty$ (a factor $(\log n)^{-1 / 4}$ should be included for $d=4$ ), where $\omega$ is an $n$-step self-avoiding walk. The scaling limit is believed not to depend on $\lambda \in(0,1]$ in any important way. This limit is conjectured to be $\mathrm{SLE}_{8 / 3}$ for $d=2$, and the limit is not understood for $d=3$. For $d=4$, the scaling limit is believed to be Brownian motion, and for $d \geq 5$, the lace expansion has been used to prove that the scaling limit is Brownian motion [97, 98].

The special role of $d=4$ for the asymptotics of the self-avoiding walk is summarized by saying that $d=4$ is the upper critical dimension, and that mean-field behaviour applies when $d>4$. Above $d=4$, the self-avoiding walk has the same leading asymptotics as the simple random walk. Logarithmic corrections to simple random walk behaviour occur when $d=4$, and different power laws appear for $d<4$.

The critical nature of $d=4$ can be guessed from the fact that Brownian motion is 2-dimensional. Since two 2-dimensional sets generically do not intersect in more than $4=2+2$ dimensions, above four dimensions the selfavoidance constraint does not play an important role.

\subsection{Differential Inequalities and the Bubble Condition}

We now define the bubble condition and show that it is a sufficient condition for a particular critical exponent (namely $\gamma$ ) to exist and take its mean-field value. This is a useful precursor to the lace expansion. It is also a useful precursor to the study of lattice trees and percolation, where the bubble condition will be replaced by the square and triangle conditions, respectively.

For simplicity, we restrict attention in this section to the strictly selfavoiding walk with $\lambda=1$. We fix $\Omega$ to be either (1.1) or (1.2).

The bubble diagram is defined by

$$
B(z)=\sum_{x \in \mathbb{Z}^{d}} G_{z}(x)^{2} .
$$

The name "bubble diagram" comes from a Feynman diagram notation in which the two-point function evaluated at vertices $x$ and $y$ is denoted by a line terminating at $x$ and $y$. In this notation, 


$$
B(z)=\sum_{x \in \mathbb{Z}^{d}} 0 \bigcirc x=0 \bigcirc
$$

where in the diagram on the right it is implicit that the unlabelled vertex is summed over $\mathbb{Z}^{d}$. The bubble diagram can be rewritten in terms of the Fourier transform of the two-point function, using (2.30) and the Parseval relation, as

$$
B(z)=\left\|G_{z}\right\|_{2}^{2}=\left\|\hat{G}_{z}\right\|_{2}^{2}=\int_{[-\pi, \pi]^{d}} \hat{G}_{z}(k)^{2} \frac{\mathrm{d}^{d} k}{(2 \pi)^{d}} .
$$

The bubble condition is the statement that $B\left(z_{c}\right)<\infty$. In other words, the bubble condition states that $\hat{G}_{z_{c}}(k)$ is square integrable. Recall that square integrability of $\hat{C}_{1 /|\Omega|}(k)$ was important in Exercise 1.7 .

In view of the definition of $\eta$ in (2.27) or (2.28), it follows from (2.31) that the bubble condition is satisfied provided $\eta>(4-d) / 2$. Hence the bubble condition for $d>4$ is implied by the infrared bound $\eta \geq 0$. If the values for $\eta$ arising from Fisher's relation and the conjectured values of $\gamma$ and $\nu$ are correct, then the bubble condition will not hold in dimensions 2,3 or 4 , with the divergence of the bubble diagram being only logarithmic in four dimensions.

Throughout these notes,

$$
f(z) \simeq g(z) \text { denotes } \quad c^{-1} g(z) \leq f(z) \leq c g(z)
$$

for some $c>0$, uniformly in $z<z_{c}$. In this section, we prove a differential inequality for the susceptibility, which shows that the bubble condition implies that $\gamma=1$ in the sense that $\chi(z) \simeq\left(1-z / z_{c}\right)^{-1}$. In fact, the lower bound

$$
\chi(z) \geq \frac{z_{c}}{z_{c}-z}
$$

is an immediate consequence of (2.19) and the subadditivity bound $c_{n} \geq \mu^{n}=$ $z_{c}^{-n}$, and holds with or without the bubble condition. It remains to prove that the complementary upper bound

$$
\chi(z) \leq \operatorname{const} \frac{1}{z_{c}-z}
$$

is a consequence of the bubble condition. This will be shown in the following theorem. In Section 5, we will use the lace expansion to prove the bubble condition for the spread-out model (1.2) for $d>4$ with $L$ sufficiently large, and for the nearest-neighbour model with $d$ sufficiently large.

A version of Theorem 2.3 was proved in [36]. The role of the bubble condition in proving mean-field behaviour for spin systems in dimensions $d>4$ was developed previously, in [3, 76, 192] (see also [73]). In Section 5.4, it will be shown that the lace expansion actually provides a differential equality in place of the differential inequalities of Theorem 2.3. 
Theorem 2.3. For $0<z<z_{c}$, the susceptibility obeys the differential inequalities

$$
\frac{\chi(z)^{2}}{B(z)} \leq \frac{\mathrm{d}}{\mathrm{d} z}[z \chi(z)] \leq \chi(z)^{2}
$$

and the inequalities

$$
\frac{z_{c}}{z_{c}-z} \leq \chi(z) \leq B\left(z_{c}\right) \frac{2 z_{c}-z}{z_{c}-z} .
$$

Thus the bubble condition implies that $\chi(z) \simeq\left(1-z / z_{c}\right)^{-1}$, which is to say that $\gamma$ exists and equals 1.

Proof. We first prove the differential inequalities (2.35). By definition,

$$
\chi(z)=\sum_{n=0}^{\infty} c_{n} z^{n}=\sum_{y} \sum_{\omega \in \mathcal{S}(0, y)} z^{|\omega|}
$$

where $|\omega|$ denotes the number of steps in $\omega$. For $0<z<z_{c}$, term by term differentiation gives

$$
Q(z)=\frac{\mathrm{d}}{\mathrm{d} z}[z \chi(z)]=\sum_{y} \sum_{\omega \in \mathcal{S}(0, y)}(|\omega|+1) z^{|\omega|},
$$

where the first equality defines $Q(z)$. This can be rewritten as

$$
\begin{aligned}
Q(z) & =\sum_{y} \sum_{\omega \in \mathcal{S}(0, y)} \sum_{x} I[\omega(j)=x \text { for some } j] z^{|\omega|} \\
& =\sum_{x, y} \sum_{\substack{\omega^{(1)} \in \mathcal{S}(0, x) \\
\omega^{(2)} \in \mathcal{S}(x, y)}} z^{\left|\omega^{(1)}\right|+\left|\omega^{(2)}\right|} I\left[\omega^{(1)} \cap \omega^{(2)}=\{x\}\right],
\end{aligned}
$$

where $I$ denotes the indicator function.

If we ignore the mutual avoidance of $\omega^{(1)}$ and $\omega^{(2)}$ in (2.39), we obtain the upper bound

$$
\frac{\mathrm{d}(z \chi(z))}{\mathrm{d} z} \leq \chi(z)^{2}
$$

of $(2.35)$.

To obtain a complementary bound, we rewrite $Q(z)$ by using the inclusionexclusion relation in the form

$$
I\left[\omega^{(1)} \cap \omega^{(2)}=\{x\}\right]=1-I\left[\omega^{(1)} \cap \omega^{(2)} \neq\{x\}\right] .
$$

This gives

$$
Q(z)=\chi(z)^{2}-\sum_{x, y} \sum_{\substack{\omega^{(1)} \in \mathcal{S}(0, x) \\ \omega^{(2)} \in \mathcal{S}(x, y)}} z^{\left|\omega^{(1)}\right|+\left|\omega^{(2)}\right|} I\left[\omega^{(1)} \cap \omega^{(2)} \neq\{x\}\right] .
$$


Since $x \in \omega^{(1)} \cap \omega^{(2)}$, the indicator forces a nontrivial intersection. In the last term on the right hand side of $(2.41)$, let $w=\omega^{(2)}(l)$ be the site of the last intersection of $\omega^{(2)}$ with $\omega^{(1)}$, where time is measured along $\omega^{(2)}$ beginning at its starting point $x$. Then the portion of $\omega^{(2)}$ corresponding to times greater than $l$ must avoid all of $\omega^{(1)}$. Relaxing the restrictions that this portion of $\omega^{(2)}$ avoid both the remainder of $\omega^{(2)}$ and the part of $\omega^{(1)}$ linking $w$ to $x$, and also relaxing the mutual avoidance of the two portions of $\omega^{(1)}$, gives the upper bound

$$
\sum_{x, y} \sum_{\substack{\omega^{(1)} \in \mathcal{S}(0, x) \\ \omega^{(2)} \in \mathcal{S}(x, y)}} z^{\left|\omega^{(1)}\right|+\left|\omega^{(2)}\right|} I\left[\omega^{(1)} \cap \omega^{(2)} \neq\{x\}\right] \leq Q(z)[B(z)-1],
$$

as illustrated in Fig. 2.2. Here the factor $B(z)-1$ arises from the two paths joining $w$ and $x$. The upper bound involves $B(z)-1$ rather than $B(z)$ since there will be no contribution from the $x=0$ term in (2.30).

Exercise 2.4. Convince yourself that (2.42) is correct.

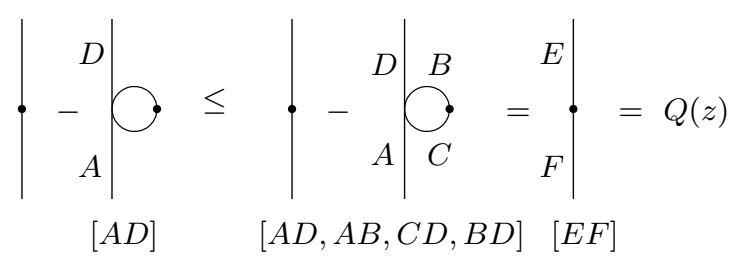

Fig. 2.2. A diagrammatic representation of the inequality $\chi(z)^{2}-Q(z)[B(z)-$ $1] \leq Q(z)$. The lists of pairs of lines indicate interactions, in the sense that the corresponding walks must avoid each other.

Combining (2.41) and (2.42) gives

$$
Q(z) \geq \chi(z)^{2}-Q(z)[B(z)-1] .
$$

Solving for $Q(z)$ gives

$$
Q(z) \geq \frac{\chi(z)^{2}}{B(z)}
$$

which is the lower bound of (2.35).

Next, we show that (2.35) implies (2.36). The lower bound of (2.36) has already been established in (2.33) (and also follows by integration of the upper bound of (2.35)). To obtain the upper bound of (2.36) from the lower bound of $(2.35)$, we proceed as follows. Let $z_{1} \in\left[0, z_{c}\right)$. The lower bound of $(2.35)$ implies that, for $z \in\left[z_{1}, z_{c}\right)$, 


$$
z\left(-\frac{d \chi^{-1}}{d z}\right) \geq \frac{1}{B(z)}-\frac{1}{\chi(z)} \geq \frac{1}{B\left(z_{c}\right)}-\frac{1}{\chi\left(z_{1}\right)},
$$

where $\chi^{-1}$ denotes the reciprocal. We bound the factor of $z$ on the left hand side by $z_{c}$ and then integrate from $z_{1}$ to $z_{c}$. Using the fact that $\chi\left(z_{c}\right)^{-1}=0$ by $(2.33)$, this gives

$$
z_{c} \chi\left(z_{1}\right)^{-1} \geq\left[B\left(z_{c}\right)^{-1}-\chi\left(z_{1}\right)^{-1}\right]\left(z_{c}-z_{1}\right) .
$$

Rewriting gives the upper bound of (2.36).

By $(2.39), Q(z)$ is the generating function for pairs of self-avoiding walks which do not intersect each other apart from their common starting point. It follows from Theorem 2.3 that if the bubble condition holds then

$$
\frac{Q(z)}{\chi(z)^{2}} \simeq 1
$$

a relation related to the non-vanishing of the non-intersection probability $q_{n}$ of (2.12), as $n \rightarrow \infty$, when $\gamma=1$.

\section{The Lace Expansion for the Self-Avoiding Walk}

The lace expansion was derived by Brydges and Spencer in [45]. Their derivation, which is given below in Sections 3.2-3.3, involves an expansion and resummation procedure closely related to the cluster expansions of statistical mechanics [40]. It was later noted that the lace expansion can also be seen as resulting from repeated application of the inclusion-exclusion relation [186]. For a more combinatorial description of the lace expansion, see [211]. We first discuss the inclusion-exclusion approach.

\subsection{Inclusion-Exclusion}

The inclusion-exclusion approach to the lace expansion is closely related to the method of proof of Theorem 2.3. In that proof, a single inclusion-exclusion was used to obtain upper and lower bounds. Here, we will derive an identity by using repeated inclusion-exclusion.

For simplicity, we restrict attention to the strictly self-avoiding walk $(\lambda=$ 1 ). We consider a walk taking steps in a finite set $\Omega$, so that $\omega(i+1)-\omega(i) \in \Omega$ for each $i$, but there is no need here for a symmetry assumption and $\Omega$ is an arbitrary finite set. As in (1.10), we write

$$
D(x)=\frac{1}{|\Omega|} I[x \in \Omega]
$$

We rewrite $c_{n}(x)$ using the inclusion-exclusion relation. Namely, we first count all walks from 0 to $x$ which are self-avoiding after the first step, and then 
subtract the contribution due to those which are not self-avoiding from the beginning, i.e., walks that return to the origin. Since $c_{1}(0, y)=1$ for $y \in \Omega$, this gives

$$
c_{n}(x)=\left(c_{1} * c_{n-1}\right)(x)-\sum_{y \in \Omega} \sum_{\omega^{(1)} \in \mathcal{S}_{n-1}(y, x)} I\left[0 \in \omega^{(1)}\right] .
$$

Comparing with (1.5), it is the second term on the right hand side that makes the above equation interesting.

The inclusion-exclusion relation can now be applied to the last term of (3.2), as follows. Let $s$ be the first (and only) time that $\omega^{(1)}(s)=0$. Then for $y \in \Omega$,

$$
\begin{aligned}
& \sum_{\omega^{(1)} \in \mathcal{S}_{n-1}(y, x)} I\left[0 \in \omega^{(1)}\right] \\
= & \sum_{s=1}^{n-1} \sum_{\substack{\omega^{(2)} \in \mathcal{S}_{s}(y, 0) \\
\omega^{(3)} \in \mathcal{S}_{n-1-s}(0, x)}} I\left[\omega^{(2)} \cap \omega^{(3)}=\{0\}\right] \\
= & \sum_{s=1}^{n-1}\left[c_{s}(y, 0) c_{n-1-s}(0, x)-\sum_{\substack{\omega^{(2)} \in \mathcal{S}_{s}(y, 0) \\
\omega^{(3)} \in \mathcal{S}_{n-1-s}(0, x)}} I\left[\omega^{(2)} \cap \omega^{(3)} \neq\{0\}\right]\right] .
\end{aligned}
$$

We can interpret $c_{s}(y, 0)$ as the number of $(s+1)$-step walks which step from the origin directly to $y$, then return to the origin in $s$ steps, and which have distinct vertices apart from the fact that they return to their starting point. Let $\mathcal{U}_{s}$ denote the set of all $s$-step self-avoiding loops at the origin $(s$-step walks which begin and end at the origin but which otherwise have distinct vertices), and let $u_{s}$ be the cardinality of $\mathcal{U}_{s}$. Then

$$
\begin{aligned}
& \sum_{y \in \Omega} \sum_{\omega^{(1)} \in \mathcal{S}_{n-1}(y, x)} I\left[0 \in \omega^{(1)}\right] \\
& =\sum_{s=2}^{n} u_{s} c_{n-s}(x)-\sum_{\substack{s=2 \\
\omega^{(3)} \in \mathcal{S}_{n-s}(0, x)}} \sum_{\substack{(2) \in \mathcal{U}_{s} \\
\omega^{\prime}}} I\left[\omega^{(2)} \cap \omega^{(3)} \neq\{0\}\right] .
\end{aligned}
$$

Continuing in this fashion, in the last term on the right hand side of the above equation, let $t \geq 1$ be the first time along $\omega^{(3)}$ that $\omega^{(3)}(t) \in \omega^{(2)}$, and let $v=\omega^{(3)}(t)$. Then the inclusion-exclusion relation can be applied again to remove the avoidance between the portions of $\omega^{(3)}$ before and after $t$, and correct for this removal by the subtraction of a term involving a further intersection. Repetition of this procedure leads to the convolution equation

$$
c_{n}(0, x)=\left(|\Omega| D * c_{n-1}\right)(x)+\sum_{m=2}^{n}\left(\pi_{m} * c_{n-m}\right)(x),
$$


where we have used $c_{1}(x)=|\Omega| D(x)$, and where $\pi_{m}$ is given by

$$
\pi_{m}(v)=\sum_{N=1}^{\infty}(-1)^{N} \pi_{m}^{(N)}(v)
$$

with the terms on the right hand side defined as follows. The $N=1$ term is given by

$$
\pi_{m}^{(1)}(v)=\delta_{0, v} u_{m}=\delta_{0, v} \quad 0 \bigcirc
$$

where the diagram represents $u_{m}$. The $N=2$ term is

$$
\pi_{m}^{(2)}(v)=\sum_{\substack{m_{1}, m_{2}, m_{3}: \\ m_{1}+m_{2}+m_{3}=m}} \sum_{\substack{\omega_{1} \in \mathcal{S}_{m_{1}}(0, v) \\ \sum_{\omega_{2}} \in \mathcal{S}_{m_{2}}(v, 0)}} \sum_{\omega_{3} \in \mathcal{S}_{m_{3}}(0, v)} I\left(\omega_{1}, \omega_{2}, \omega_{3}\right),
$$

where $I\left(\omega_{1}, \omega_{2}, \omega_{3}\right)$ is equal to 1 if the $\omega_{i}$ are pairwise mutually avoiding apart from their common endpoints, and otherwise equals 0. Diagrammatically this can be represented by

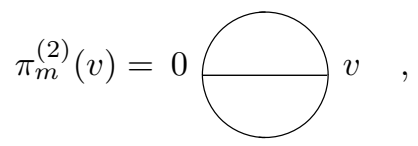

where each line represents a sum over self-avoiding walks between the endpoints of the line, with mutual avoidance between the three pairs of lines in the diagram. Similarly

$$
\pi_{m}^{(3)}(v)=\overbrace{0}>_{v}
$$

where now there is mutual avoidance between some but not all pairs of lines in the diagram; a precise description requires some care. The unlabelled vertex is summed over $\mathbb{Z}^{d}$. A slashed diagram line is used to indicate a walk which may have zero steps, i.e., be a single site, whereas lines without a slash correspond to walks of at least one step. All the higher order terms can be expressed as diagrams in this way, and with some care it is possible to keep track of the pattern of mutual avoidance between subwalks (individual lines in the diagram) which emerges. The algebraic derivation of the expansion, described next, keeps track of this mutual avoidance automatically. Equations (3.5)(3.6) constitute the lace expansion. No laces have appeared yet, but they will come later.

Exercise 3.1. Determine a precise expression for $\pi_{m}^{(3)}(v)$. What is the picture for $\pi_{m}^{(4)}(v)$ ? 


\subsection{Expansion}

In this and the following section, we give the original derivation of the lace expansion due to Brydges and Spencer [45]. The expansion applies in a more general context than we have considered so far, and we will give a quite general derivation.

Consider walks taking steps in a finite subset $\Omega \subset \mathbb{Z}^{d}$. Suppose that to each walk $\omega=(\omega(0), \omega(1), \ldots, \omega(n))$ and each pair $s, t \in\{0,1, \ldots, n\}$, we are given a complex number $\mathcal{U}_{s t}(\omega)$ (for example, (2.1)).

Definition 3.2. (i) Given an interval $I=[a, b]$ of positive integers, we refer to a pair $\{s, t\}(s<t)$ of elements of I as an edge. To abbreviate the notation, we usually write st for $\{s, t\}$. A set of edges is called a graph. The set of all graphs on $[a, b]$ is denoted $\mathcal{B}[a, b]$.

(ii) A graph $\Gamma$ is said to be connected if both $a$ and $b$ are endpoints of edges in $\Gamma$, and if in addition, for any $c \in(a, b)$, there are $s, t \in[a, b]$ such that $s<$ $c<t$ and $s t \in \Gamma$. In other words, $\Gamma$ is connected if, as intervals, $\cup_{s t \in \Gamma}(s, t)=$ $(a, b)$. The set of all connected graphs on $[a, b]$ is denoted $\mathcal{G}[a, b]$.

An apology is required for graph theorists. The above notion of connectivity is not the usual notion of path-connectivity in graph theory. Instead, the above notion relies heavily on the fact that the vertices of the graph are linearly ordered in time, and may be justified by the fact that connected graphs are those for which $\cup_{s t \in \Gamma}(s, t)$ is equal to the connected interval $(a, b)$. In any event, it is decidedly not path-connectivity. There are connected graphs that are not path-connected, and vice versa. It is convenient to have in mind the representation of graphs illustrated in Fig. 3.1.

(a)

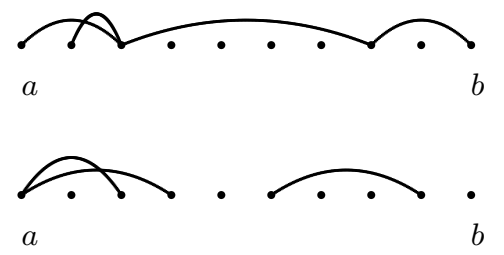

(b)
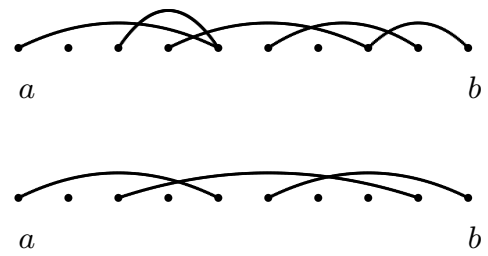

Fig. 3.1. Graphs in which an edge $s t$ is represented by an arc joining $s$ and $t$. The graphs in (a) are not connected, whereas the graphs in (b) are connected. 
We set $K[a, a]=1$, and for $a<b$ we define

$$
K[a, b]=\prod_{a \leq s<t \leq b}\left(1+\mathcal{U}_{s t}\right),
$$

where the dependence on $\omega$ is left implicit. By expanding the product in (3.7), we obtain

$$
K[a, b]=\sum_{\Gamma \in \mathcal{B}[a, b]} \prod_{s t \in \Gamma} \mathcal{U}_{s t} .
$$

Note that $\mathcal{B}[a, b]$ contains the graph with no edges, so our convention that $K[a, a]=1$ is consistent with the standard convention that an empty product is equal to 1 .

Exercise 3.3. Prove (3.8).

We set $J[a, a]=1$, and for $a<b$ we define a quantity analogous to $K[a, b]$, but with the sum over graphs restricted to connected graphs:

$$
J[a, b]=\sum_{\Gamma \in \mathcal{G}[a, b]} \prod_{s t \in \Gamma} \mathcal{U}_{s t} .
$$

Lemma 3.4. For any $a<b$,

$$
K[a, b]=K[a+1, b]+\sum_{j=a+1}^{b} J[a, j] K[j, b] .
$$

Proof. The contribution to the sum on the right hand side of (3.8) due to all graphs $\Gamma$ for which $a$ is not in an edge is exactly $K[a+1, b]$. To resum the contribution due to the remaining graphs, we proceed as follows. If $\Gamma$ does contain an edge containing $a$, let $j(\Gamma)$ be the largest value of $j$ such that the set of edges in $\Gamma$ with both ends in the interval $[a, j]$ forms a connected graph on $[a, j]$. Then the sum over $\Gamma$ factorizes into sums over connected graphs on $[a, j]$ and arbitrary graphs on $[j, b]$, and resummation of the latter gives

$$
K[a, b]=K[a+1, b]+\sum_{j=a+1}^{b} \sum_{\Gamma \in \mathcal{G}[a, j]} \prod_{s t \in \Gamma} \mathcal{U}_{s t} K[j, b],
$$

which with (3.9) proves the lemma.

Let

$$
c_{n}(x)=\sum_{\omega \in \mathcal{W}_{n}(x)} K[0, n]=\sum_{\omega \in \mathcal{W}_{n}(x)} \prod_{0 \leq s<t \leq n}\left(1+\mathcal{U}_{s t}(\omega)\right),
$$

a generalization of (2.2). It is simplest if we assume that $\mathcal{U}_{s t}(\omega)$ is invariant under spatial translation of $\omega$, and under an equal shift of each of $s, t$ and the time parameter of $\omega$, and we make this assumption. Note that (2.1) obeys the 
assumption. We substitute (3.10) into (3.12). A key point is that in the last term of (3.10) the portion of the walk from time $j$ onwards is independent of the portion up to time $j$. Let

$$
\pi_{m}(x)=\sum_{\omega \in \mathcal{W} \mathcal{W}_{m}(0, x)} J[0, m] .
$$

Then for $n \geq 1$, we obtain

$$
c_{n}(x)=\left(|\Omega| D * c_{n-1}\right)(x)+\sum_{m=1}^{n}\left(\pi_{m} * c_{n-m}\right)(x),
$$

as in (3.5). ${ }^{1}$ To obtain a more useful representation of $\pi_{m}$ than (3.13), we perform a resummation of (3.13) using the notion of laces.

\subsection{Laces and Resummation}

Definition 3.5. A lace is a minimally connected graph, i.e., a connected graph for which the removal of any edge would result in a disconnected graph. The set of laces on $[a, b]$ is denoted by $\mathcal{L}[a, b]$, and the set of laces on $[a, b]$ which consist of exactly $N$ edges is denoted $\mathcal{L}^{(N)}[a, b]$.

We write $L \in \mathcal{L}^{(N)}[a, b]$ as $L=\left\{s_{1} t_{1}, \ldots, s_{N} t_{N}\right\}$, with $s_{l}<t_{l}$ for each $l$. The fact that $L$ is a lace is equivalent to a certain ordering of the $s_{l}$ and $t_{l}$. For $N=1$, we simply have $a=s_{1}<t_{1}=b$. For $N \geq 2, L \in \mathcal{L}^{(N)}[a, b]$ if and only if

$$
a=s_{1}<s_{2}, \quad s_{l+1}<t_{l} \leq s_{l+2} \quad(l=1, \ldots, N-2), \quad s_{N}<t_{N-1}<t_{N}=b
$$

(for $N=2$ the vacuous middle inequalities play no role); see Fig. 3.2. Thus $L$ divides $[a, b]$ into $2 N-1$ subintervals:

$$
\left[s_{1}, s_{2}\right],\left[s_{2}, t_{1}\right],\left[t_{1}, s_{3}\right],\left[s_{3}, t_{2}\right], \ldots,\left[s_{N}, t_{N-1}\right],\left[t_{N-1}, t_{N}\right] .
$$

Of these, intervals number $3,5, \ldots,(2 N-3)$ can have zero length for $N \geq 3$, whereas all others have length at least 1.

Exercise 3.6. Prove that (3.15) characterizes laces.

Given a connected graph $\Gamma \in \mathcal{G}[a, b]$, the following prescription associates to $\Gamma$ a unique lace $\mathrm{L}_{\Gamma} \subset \Gamma$ : The lace $\mathrm{L}_{\Gamma}$ consists of edges $s_{1} t_{1}, s_{2} t_{2}, \ldots$, with $t_{1}, s_{1}, t_{2}, s_{2}, \ldots$ determined, in that order, by

$$
t_{1}=\max \{t: a t \in \Gamma\}, \quad s_{1}=a,
$$

\footnotetext{
${ }^{1}$ For $m=1$, there is a single connected graph $\{01\}$, and when $\mathcal{U}_{s t}$ is given by (2.1) we have $\pi_{1}(x)=\sum_{\omega \in \mathcal{W}_{1}(0, x)} U_{01}(\omega)=0$, since it is always the case that $\omega(0) \neq \omega(1)$. Thus the sum over $m$ in (3.14) can be started at $m=2$ in this case.
} 


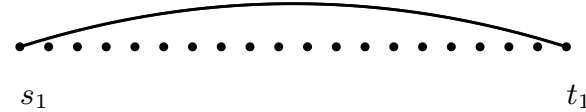

$t_{1}$
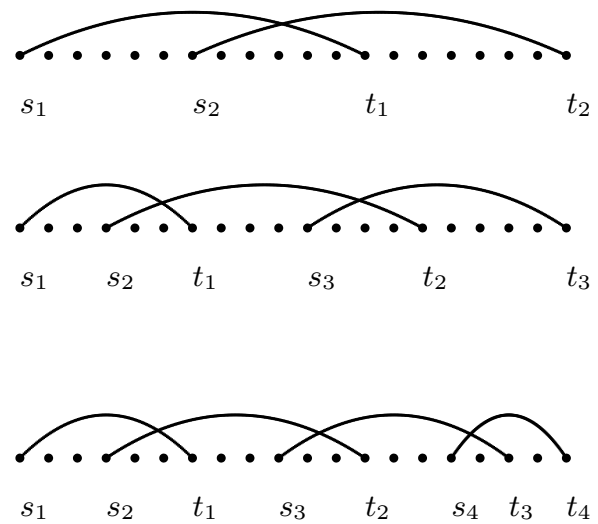

Fig. 3.2. Laces in $\mathcal{L}^{(N)}[a, b]$ for $N=1,2,3,4$, with $s_{1}=a$ and $t_{N}=b$.

$$
t_{i+1}=\max \left\{t: \exists s<t_{i} \text { such that } s t \in \Gamma\right\}, \quad s_{i+1}=\min \left\{s: s t_{i+1} \in \Gamma\right\} .
$$

The procedure terminates when $t_{i+1}=b$. Given a lace $L$, the set of all edges st $\notin L$ such that $\mathrm{L}_{L \cup\{s t\}}=L$ is denoted $\mathcal{C}(L)$. Edges in $\mathcal{C}(L)$ are said to be compatible with $L$. Fig. 3.3 illustrates these definitions.

Exercise 3.7. Show that $\mathrm{L}_{\Gamma}=L$ if and only if $L$ is a lace, $L \subset \Gamma$, and $\Gamma \backslash L \subset \mathcal{C}(L)$.

The sum over connected graphs in (3.9) can be performed by first summing over all laces and then, given a lace, summing over all connected graphs associated to that lace by the above prescription. This gives

$$
J[a, b]=\sum_{L \in \mathcal{L}[a, b]} \prod_{s t \in L} \mathcal{U}_{s t} \sum_{\Gamma: \mathrm{L}_{\Gamma}=L} \prod_{s^{\prime} t^{\prime} \in \Gamma \backslash L} \mathcal{U}_{s^{\prime} t^{\prime}}
$$

But, writing $\Gamma^{\prime}=\Gamma \backslash L$, it follows from Exercise 3.7 that

$$
\sum_{\Gamma: \mathrm{L}_{\Gamma}=L} \prod_{s^{\prime} t^{\prime} \in \Gamma \backslash L} \mathcal{U}_{s^{\prime} t^{\prime}}=\sum_{\Gamma^{\prime} \subset \mathcal{C}(L)} \prod_{s^{\prime} t^{\prime} \in \Gamma^{\prime}} \mathcal{U}_{s^{\prime} t^{\prime}}=\prod_{s^{\prime} t^{\prime} \in \mathcal{C}(L)}\left(1+\mathcal{U}_{s^{\prime} t^{\prime}}\right) .
$$

Therefore,

$$
J[a, b]=\sum_{L \in \mathcal{L}[a, b]} \prod_{s t \in L} \mathcal{U}_{s t} \prod_{s^{\prime} t^{\prime} \in \mathcal{C}(L)}\left(1+\mathcal{U}_{s^{\prime} t^{\prime}}\right)
$$


(a)

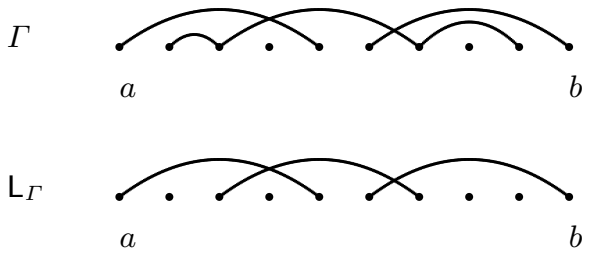

(b)

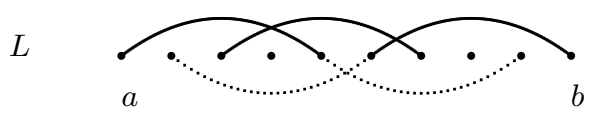

(c)

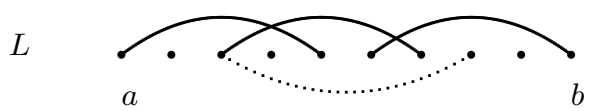

Fig. 3.3. (a) A connected graph $\Gamma$ and its associated lace $L=\mathrm{L}_{\Gamma}$. (b) The dotted edges are compatible with the lace $L$. (c) The dotted edge is not compatible with the lace $L$.

Inserting this in (3.13) gives

$$
\pi_{m}(x)=\sum_{\omega \in \mathcal{W}_{m}(0, x)} \sum_{L \in \mathcal{L}[0, m]} \prod_{s t \in L} \mathcal{U}_{s t} \prod_{s^{\prime} t^{\prime} \in \mathcal{C}(L)}\left(1+\mathcal{U}_{s^{\prime}} t^{\prime}\right)
$$

For $a<b$ we define $J^{(N)}[a, b]$ to be the contribution to (3.17) from laces consisting of exactly $N$ bonds:

$$
J^{(N)}[a, b]=\sum_{L \in \mathcal{L}^{(N)}[a, b]} \prod_{s t \in L} \mathcal{U}_{s t} \prod_{s^{\prime} t^{\prime} \in \mathcal{C}(L)}\left(1+\mathcal{U}_{s^{\prime} t^{\prime}}\right) .
$$

For the special case in which $\mathcal{U}_{s t}$ is given by (2.1), each term in the above sum is either 0 or $(-1)^{N}$. By (3.17) and (3.21),

$$
J[a, b]=\sum_{N=1}^{\infty} J^{(N)}[a, b]
$$

The sum over $N$ in (3.22) is a finite sum, since the sum in (3.21) is empty for $N>b-a$ and hence $J^{(N)}[a, b]=0$ if $N>b-a$.

Now we define

$$
\begin{aligned}
\pi_{m}^{(N)}(x) & =(-1)^{N} \sum_{\omega \in \mathcal{W}_{m}(x)} J^{(N)}[0, m] \\
& =\sum_{\omega \in \mathcal{W}_{m}(x)} \sum_{L \in \mathcal{L}^{(N)}[0, m]} \prod_{s t \in L}\left(-\mathcal{U}_{s t}\right) \prod_{s^{\prime} t^{\prime} \in \mathcal{C}(L)}\left(1+\mathcal{U}_{s^{\prime} t^{\prime}}\right) .
\end{aligned}
$$

The factor $(-1)^{N}$ on the right hand side of (3.23) has been inserted to arrange that 


$$
\pi_{m}^{(N)}(x) \geq 0 \text { for all } N, m, x
$$

when $\mathcal{U}_{s t}$ is given by $U_{s t}$ of (2.1). By (3.13), (3.22) and (3.23),

$$
\pi_{m}(x)=\sum_{N=1}^{\infty}(-1)^{N} \pi_{m}^{(N)}(x) .
$$

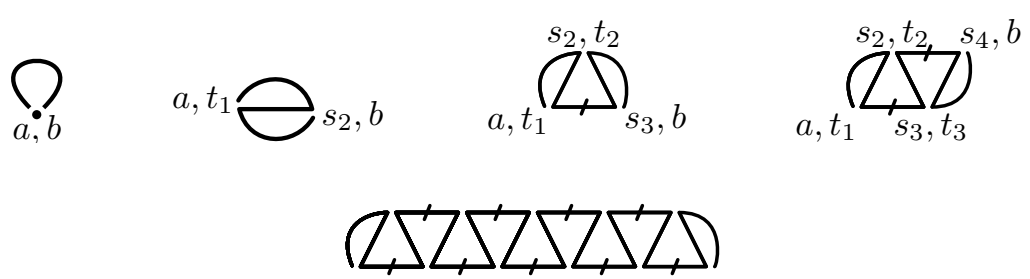

Fig. 3.4. Self-intersections required for a walk $\omega$ with $\prod_{s t \in L} U_{s t}(\omega) \neq 0$, with $U_{s t}$ given by (2.1), for the laces with $N=1,2,3,4$ bonds depicted in Fig. 3.2. The picture for $N=11$ is also shown.

For the special case in which $\mathcal{U}_{s t}$ is given by (2.1), walks making a nonzero contribution to (3.23) are constrained to have the topology indicated in Fig. 3.4. In the figure, for $\prod_{s^{\prime} t^{\prime} \in \mathcal{C}(L)}\left(1+U_{s^{\prime} t^{\prime}}\right) \neq 0$, each of the $2 N-1$ subwalks must be a self-avoiding walk, and in addition there must be mutual avoidance between some (but not all) of the subwalks. The number of loops (faces excluding the "outside" face) in a diagram is equal to the number of edges in the corresponding lace. The lines which are slashed correspond to subwalks which may consist of zero steps, but the others correspond to subwalks consisting of at least one step. This gives an interpretation of $\pi_{m}^{(N)}$ identical to that obtained in Section 3.1, but here there is the advantage that explicit formulas keep track of the mutual avoidance between subwalks.

It is sometimes convenient to modify the definitions of "connected graph" and "lace," and we will do so in Section 8.1. A more general theory of laces is developed and applied in $[124,126]$, for the analysis of networks of mutuallyavoiding self-avoiding walks. See also [125] for an application of the more general theory to lattice trees.

\subsection{Transformations}

Equation (3.14) involves convolution in both space and time. It has been studied in this form in [29], via fixed point methods.

It is tempting to use transformations to eliminate one or both of these convolutions. We can eliminate the convolution in space if we take the Fourier transform (1.6). For $n \geq 1$, this gives 


$$
\hat{c}_{n}(k)=|\Omega| \hat{D}(k) \hat{c}_{n-1}(k)+\sum_{m=1}^{n} \hat{\pi}_{m}(k) \hat{c}_{n-m}(k) .
$$

Conditions are given in [120] which ensure that solutions of (3.26) have Gaussian asymptotics, via an analysis based on induction on $n$.

We may instead prefer to eliminate the convolution in time, by going to generating functions. Using (2.18) and (3.14), this gives

$$
\begin{aligned}
G_{z}(x) & =\delta_{0, x}+\sum_{n=1}^{\infty} c_{n}(x) z^{n} \\
& =\delta_{0, x}+z|\Omega|\left(D * G_{z}\right)(x)+\left(\Pi_{z} * G_{z}\right)(x),
\end{aligned}
$$

where

$$
\Pi_{z}(x)=\sum_{m=1}^{\infty} \pi_{m}(x) z^{m} .
$$

Equation (3.27) has been studied in [90, 91].

Finally, we may prefer to eliminate both convolutions by using both the Fourier transform and generating functions. Taking the Fourier transform of (3.27) gives

$$
\hat{G}_{z}(k)=1+z|\Omega| \hat{D}(k) \hat{G}_{z}(k)+\hat{\Pi}_{z}(k) \hat{G}_{z}(k),
$$

which can be solved to give

$$
\hat{G}_{z}(k)=\frac{1}{1-z|\Omega| \hat{D}(k)-\hat{\Pi}_{z}(k)} .
$$

Equation (3.30) has been the point of departure for several studies of the self-avoiding walk, and we will work with (3.30) in Section 5.

Exercise 3.8. The memory- 2 walk is the walk with $\mathcal{U}_{s t}=U_{s t}$ if $t-s \leq 2$, and otherwise $\mathcal{U}_{s t}=0$. This is a random walk with no immediate reversals. Suppose that $0 \notin \Omega \subset \mathbb{Z}^{d}$ is finite and invariant under the symmetries of the lattice.

(a) What is the value of $\hat{c}_{n}(0)$, the number of $n$-step memory-2 walks? (Calculation is not required.)

(b) Prove that for the memory-2 walk, for $m \geq 2$,

$$
\pi_{m}(x)= \begin{cases}-|\Omega| \delta_{x, 0} & \text { if } m \text { is even } \\ I[x \in \Omega] & \text { if } m \text { is odd. }\end{cases}
$$

(c) Suppose that $|\Omega|>2$. Show that the mean-square displacement for the memory-two walk is given by

$$
\sigma^{2}\left[\left(\frac{1+\delta}{1-\delta}\right) n-\frac{2 \delta\left(1-\delta^{n}\right)}{(1-\delta)^{2}}\right] \sim\left(\frac{\sigma^{2}|\Omega|}{|\Omega|-2}\right) n,
$$


where $\sigma^{2}=\sum_{x}|x|^{2} D(x)$ is the variance of $D$ and $\delta=(|\Omega|-1)^{-1}$. One approach $^{2}$ is to use (3.26) to compute $\nabla^{2} \hat{c}_{n}(0)$. This problem goes back a long way $[63,18,72]$.

(d) Show that for the memory-two walk,

$$
\hat{G}_{z}(k)=\frac{1-z^{2}}{1+(|\Omega|-1) z^{2}-z|\Omega| \hat{D}(k)}
$$

(compare Exercise 2.2 for $d=1$ ). This formula was used to compute the mean-square displacement via contour integration in [158, Section 5.3].

The memory- $\tau$ walk is the walk with $\mathcal{U}_{s t}=U_{s t}$ if $t-s \leq \tau$, and otherwise $\mathcal{U}_{s t}=0$. Finite-memory walks played an important role in the original analysis of the lace expansion in [45], but will not concern us further here. For a study of generating functions of the number of memory- $\tau$ walks, for $\tau \leq 8$, see [171].

\section{Diagrammatic Estimates for the Self-Avoiding Walk}

The difficulty in analyzing the lace expansion is to understand the function $\pi_{m}(x)$, or one of its transforms. In this section, we will prove estimates for the Fourier transform $\hat{\Pi}_{z}(k)$ of the generating function $\Pi_{z}(x)=\sum_{m=1}^{\infty} \pi_{m}(x) z^{m}$. Related estimates of one sort or another have been used in every analysis of the lace expansion for the self-avoiding walk. Throughout this section, we use the notation of Section 3.2, and we take $\mathcal{U}_{s t}$ to be given by (2.1), i.e.,

$$
\mathcal{U}_{s t}=U_{s t}=\left\{\begin{aligned}
-1 & \text { if } \omega(s)=\omega(t) \\
0 & \text { if } \omega(s) \neq \omega(t)
\end{aligned}\right.
$$

We also assume that our walks take steps in a finite set $\Omega$ which is invariant under the symmetries of $\mathbb{Z}^{d}$, namely permutation of coordinates and replacement of any coordinate $x_{i}$ by $-x_{i}$.

We will obtain estimates for $\sum_{x \in \mathbb{Z}^{d}} \Pi_{z}^{(N)}(x)$, which is an upper bound for $\left|\hat{\Pi}_{z}^{(N)}(k)\right|$, and for $\sum_{x \in \mathbb{Z}^{d}}[1-\cos (k \cdot x)] \Pi_{z}^{(N)}(x)$, which is an upper bound for $\hat{\Pi}_{z}^{(N)}(0)-\hat{\Pi}_{z}^{(N)}(k)$. To motivate the latter, let $\hat{F}_{z}(k)=1 / \hat{G}_{z}(k)$, and note from (3.30) that

$$
\begin{aligned}
\hat{G}_{z}(k) & =\frac{1}{\hat{F}_{z}(0)+\left[\hat{F}_{z}(k)-\hat{F}_{z}(0)\right]} \\
& =\frac{1}{\hat{F}_{z}(0)+z|\Omega|[1-\hat{D}(k)]+\left[\hat{\Pi}_{z}(0)-\hat{\Pi}_{z}(k)\right]} .
\end{aligned}
$$

Our estimate for $\sum_{x \in \mathbb{Z}^{d}}[1-\cos (k \cdot x)] \Pi_{z}^{(N)}(x)$ will ultimately allow us to compare the terms $\left[\hat{\Pi}_{z}(0)-\hat{\Pi}_{z}(k)\right]$ and $z|\Omega|[1-\hat{D}(k)]$ in the denominator.

\footnotetext{
${ }^{2}$ Verification of the formula by induction seems an unsatisfactory solution, since it requires prior knowledge of the formula.
} 


\subsection{The Diagrammatic Estimates}

Recall from (3.23) and (3.25) that $\pi_{m}(x)=\sum_{N=1}^{\infty}(-1)^{N} \pi_{m}^{(N)}(x)$, with

$$
\pi_{m}^{(N)}(x)=\sum_{\omega \in \mathcal{W}_{m}(x)} \sum_{L \in \mathcal{L}^{(N)}[0, m]} \prod_{s t \in L}\left(-U_{s t}\right) \prod_{s^{\prime} t^{\prime} \in \mathcal{C}(L)}\left(1+U_{s^{\prime} t^{\prime}}\right) .
$$

For $z \geq 0$, we define the non-negative generating function

$$
\Pi_{z}^{(N)}(x)=\sum_{m=2}^{\infty} \pi_{m}^{(N)}(x) z^{m}
$$

In the above series, we omit the term involving $\pi_{1}^{(N)}(x)$ because it is always zero, since the only lace on $[0,1]$ is $L=\{01\}$, and $U_{01}=0$ since a walk cannot be at the same place at consecutive times. By (3.28), we have

$$
\Pi_{z}(x)=\sum_{N=1}^{\infty}(-1)^{N} \Pi_{z}^{(N)}(x)
$$

Let

$$
H_{z}(x)=G_{z}(x)-\delta_{0, x}=\sum_{n=1}^{\infty} c_{n}(x) z^{n} .
$$

The following theorem gives bounds on $\Pi_{z}^{(N)}$ in terms of norms of $G_{z}$ and $H_{z}$.

Theorem 4.1. For all $z \geq 0$,

$$
\sum_{x \in \mathbb{Z}^{d}} \Pi_{z}^{(1)}(x) \leq z|\Omega|\left\|H_{z}\right\|_{\infty}
$$

and

$$
\sum_{x \in \mathbb{Z}^{d}}[1-\cos (k \cdot x)] \Pi_{z}^{(1)}(x)=0 .
$$

For $z \geq 0$ and $N \geq 2$,

$$
\sum_{x \in \mathbb{Z}^{d}} \Pi_{z}^{(N)}(x) \leq\left\|H_{z}\right\|_{\infty}\left\|H_{z} * G_{z}\right\|_{\infty}^{N-1},
$$

and

$$
\begin{aligned}
& \sum_{x \in \mathbb{Z}^{d}}[1-\cos (k \cdot x)] \Pi_{z}^{(N)}(x) \\
& \quad \leq(N+1)\lfloor N / 2\rfloor\left\|[1-\cos (k \cdot x)] H_{z}(x)\right\|_{\infty}\left\|H_{z} * G_{z}\right\|_{\infty}^{N-1} .
\end{aligned}
$$


We refer to the bounds of Theorem 4.1 as diagrammatic estimates, as they are inspired by the diagrams of Fig. 3.4. Moreover, the diagrams themselves provide a pictorial representation of the bounds, and have the dual interpretation of depicting both walk trajectories that contribute to $\Pi_{z}^{(N)}(x)$ as well as upper bounds on these quantities. See Fig. 4.1. ${ }^{3}$

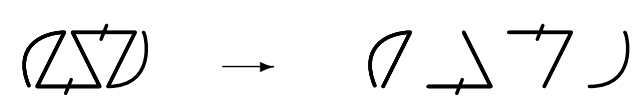

Fig. 4.1. Depiction of the estimate $\sum_{x} \Pi_{z}^{(4)}(x) \leq\left\|H_{z} * H_{z}\right\|_{\infty}\left\|H_{z} * G_{z}\right\|_{\infty}^{2}\left\|H_{z}\right\|_{\infty}$ by decomposition of the diagram for $\Pi_{z}^{(4)}$.

In applying Theorem 4.1, we will use the estimate

$$
\left\|H_{z} * G_{z}\right\|_{\infty}=\left\|H_{z}+\left(H_{z} * H_{z}\right)\right\|_{\infty} \leq\left\|H_{z}\right\|_{\infty}+\left\|H_{z}\right\|_{2}^{2},
$$

using the triangle and Cauchy-Schwarz inequalities in the last step. Since $G_{z}(0)=1$, it follows from (4.6) and (2.30) that

$$
\left\|H_{z}\right\|_{2}^{2}=\left\|G_{z}\right\|_{2}^{2}-1=B(z)-1 .
$$

To control the sum over $N$ in (4.5) using (4.9), our method in Section 5 will require, in particular, that $B\left(z_{c}\right)-1$ be small. This is a restrictive form of the bubble condition of Section 2.2 .

\subsection{Proof of the Diagrammatic Estimates}

In this section, we prove Theorem 4.1.

\subsubsection{Proof of (4.7)-(4.8)}

The estimates (4.7)-(4.8) are easy, and we prove them first. Since the unique lace on $[0, m]$ consisting of a single bond is simply the bond $0 m$, it follows from (3.23) that

$$
\pi_{m}^{(1)}(x)=\delta_{0, x} \sum_{\omega \in \mathcal{W}_{m}(0,0)} \prod_{s^{\prime} t^{\prime} \in \mathcal{C}(0 m)}\left(1+U_{s^{\prime} t^{\prime}}\right)
$$

There is no 1-step walk from 0 to 0 , so this is nonzero only for $m \geq 2$. Since $\mathcal{C}(0 m) \supset \mathcal{B}[0, m-1]$ for $m \geq 2$, it follows from (4.1) that

\footnotetext{
${ }^{3}$ Fig. 4.1 shows a slight improvement of (4.9) (as $H_{z} \leq G_{z}$ ) and it is possible to prove the improvement, but we will only prove (4.9), which suffices for our needs.
} 


$$
\begin{aligned}
0 \leq \pi_{m}^{(1)}(x) & \leq \delta_{0, x} \sum_{\omega \in \mathcal{W}_{m}(0,0)} K[0, m-1] \\
& =\delta_{0, x} \sum_{y \in \Omega} c_{m-1}(y) .
\end{aligned}
$$

Therefore, after multiplying by $z^{m}$ and summing over $m \geq 2$, we obtain

$$
0 \leq \Pi_{z}^{(1)}(x) \leq \delta_{0, x} z \sum_{y \in \Omega} H_{z}(y)
$$

which immediately implies (4.7)-(4.8).

\subsubsection{The Diagrams}

In preparation for the proof of (4.9)-(4.10), we now prove a preliminary estimate on $\Pi_{z}^{(N)}(x)$.

For $N \geq 2$, we define $p_{m}^{(N)}(x, y)$ inductively as follows. Let

$$
\begin{aligned}
p_{m}^{(2)}(x, y) & =\sum_{0<i<j \leq m} c_{i}(x) c_{j-i}(x) c_{m-j}(y) \quad(m \geq 2), \\
a_{m}(u, v, x, y) & =\delta_{v, x} \sum_{l=1}^{m} c_{l}(u-v) c_{m-l}(y-u) \quad(m \geq 1) .
\end{aligned}
$$

For $m=0,1$, we set $p_{m}^{(2)}(x, y)=0$, and we set $a_{0}(u, v, x, y)=0$. For $N \geq 3$, let

$$
p_{m}^{(N)}(x, y)=\sum_{u, v \in \mathbb{Z}^{d}} \sum_{i=0}^{m} p_{i}^{(N-1)}(u, v) a_{m-i}(u, v, x, y) \quad(m \geq 2) .
$$

For $N \geq 2$, we also define the generating functions

$$
\begin{aligned}
P_{z}^{(N)}(x, y) & =\sum_{m=2}^{\infty} p_{m}^{(N)}(x, y) z^{m}, \\
A_{z}(u, v, x, y) & =\sum_{m=1}^{\infty} a_{m}(u, v, x, y) z^{m} \\
& =\delta_{v, x} H_{z}(u-v) G_{z}(y-u) .
\end{aligned}
$$

It follows from (4.18) that, for $N \geq 3$,

$$
P_{z}^{(N)}(x, y)=\sum_{u, v \in \mathbb{Z}^{d}} P_{z}^{(N-1)}(u, v) A_{z}(u, v, x, y) .
$$

The diagrammatic representations for $P_{z}^{(N)}(x, y)$ shown in Fig. 4.2 are closely related to the diagrams appearing in Fig. 3.4. 


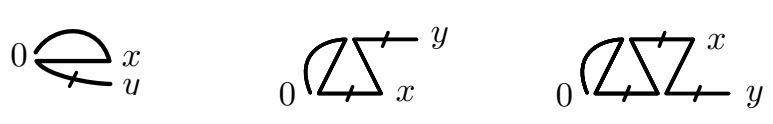

Fig. 4.2. $P_{z}^{(N)}(x, y)$ for $N=2,3,4$.

Proposition 4.2. For $N \geq 2, m \geq 2$, and $z \geq 0$,

$$
\pi_{m}^{(N)}(x) \leq p_{m}^{(N)}(x, x)
$$

and

$$
\Pi_{z}^{(N)}(x) \leq P_{z}^{(N)}(x, x) .
$$

Proof. We prove the first inequality, as the second then follows immediately from (4.4).

For $N \geq 2$ we write a lace $L \in \mathcal{L}^{(N-1)}[0, j]$ as $\left\{s_{1} t_{1}, \ldots, s_{N-1} t_{N-1}\right\}$, with $s_{1}=0$ and $t_{N-1}=j$. For $N \geq 2$, we define

$$
\begin{aligned}
& J_{x}^{(N)}[0, m] \\
& =\sum_{j=0}^{m} K[j, m] \sum_{L \in \mathcal{L}^{(N-1)}[0, j]} \sum_{i=t_{N-2}}^{j-1} \delta_{x, \omega(i)} \prod_{s t \in L}\left(-U_{s t}\right) \prod_{s^{\prime} t^{\prime} \in \mathcal{C}(L)}\left(1+U_{s^{\prime} t^{\prime}}\right),
\end{aligned}
$$

where we set $t_{0}=1$ when $N=2$. We first show that, for every $\omega$ and every $N \geq 2$,

$$
0 \leq(-1)^{N} J^{(N)}[0, m] \leq J_{\omega(m)}^{(N)}[0, m] .
$$

The first inequality is immediate, and we concentrate on the second. For this, comparing with (3.21), $L$ in (4.24) corresponds to $L \backslash\left\{s_{N} t_{N}\right\}$ in (3.21), and $i$ and $j$ of (4.24) correspond to $s_{N}$ and $t_{N-1}$ of the lace $L$ in (3.21). The set of compatible edges in (3.21) contains $\mathcal{C}\left(s_{1} t_{1}, \ldots, s_{N-1} t_{N-1}\right) \cup \mathcal{B}\left[t_{N-1}, m\right]$, and omitting factors in the product over $s^{\prime} t^{\prime}$ in (3.21) can only increase the product. When $x=\omega(m)$, the factor $\delta_{x, \omega(i)}$ is $-U_{i, m}=-U_{s_{N} t_{N}}$. This leads to $(4.25)$.

For $N \geq 2$, we define

$$
\pi_{m}^{(N)}(x, y)=\sum_{\omega \in \mathcal{W}_{m}(0, y)} J_{x}^{(N)}[0, m] .
$$

It follows from (3.23) and (4.25) that

$$
\pi_{m}^{(N)}(x) \leq \pi_{m}^{(N)}(x, x) .
$$

We will show that

$$
\pi_{m}^{(N)}(x, y) \leq p_{m}^{(N)}(x, y) \quad(N \geq 2),
$$


which then gives the proposition.

The proof of (4.28) is by induction on $N$. We begin the induction with the case $N=2$. In this case, the sum over $L$ in (4.24) consists of the single term $L=\{0 j\}$. Since $\mathcal{C}(0 j) \supset \mathcal{B}[0, i] \cup \mathcal{B}[i, j]$ for $0<i<j$, using symmetry we obtain

$$
\begin{aligned}
\pi_{m}^{(2)}(x, y) & \leq \sum_{0<i<j<m} \sum_{\omega \in \mathcal{W}_{m}(0, y)} K[0, i] \delta_{x, \omega(i)} K[i, j] \delta_{0, \omega(j)} K[j, m] \\
& =\sum_{0<i<j<m} c_{i}(x) c_{j-i}(x) c_{m-j}(y) \\
& \leq p_{m}^{(2)}(x, y) .
\end{aligned}
$$

To advance the induction, we fix $N \geq 3$ and assume that (4.28) holds for $N-1$. We replace the factor $-U_{S_{N-1} t_{N-1}}$ in the first product of (4.24) by

$$
-U_{s_{N-1} t_{N-1}}=\delta_{\omega\left(s_{N-1}\right), \omega\left(t_{N-1}\right)}=\sum_{u \in \mathbb{Z}^{d}} \delta_{\omega\left(s_{N-1}\right), u} \delta_{u, \omega\left(t_{N-1}\right)} .
$$

Given $L \in \mathcal{L}^{(N-1)}[0, j]$, let $L^{\prime}=L \backslash\left\{s_{N-1} t_{N-1}\right\}$. For $t_{N-2} \leq i<j=t_{N-1}$, we then have $\mathcal{C}(L) \supset \mathcal{C}\left(L^{\prime}\right) \cup \mathcal{B}\left[t_{N-2}, i\right] \cup \mathcal{B}[i, j]$. Using (4.30), we conclude from (4.24) that

$$
J_{x}^{(N)}[0, m] \leq \sum_{u} \sum_{0 \leq i<j \leq m} J_{u}^{(N-1)}[0, i] K[i, j] \delta_{\omega(i), x} K[j, m] \delta_{u, \omega(j)} .
$$

Therefore, recalling (4.17) and using the induction hypothesis,

$$
\begin{aligned}
\pi_{m}^{(N)}(x, y) & \leq \sum_{u} \sum_{0 \leq i<j \leq m} \pi_{i}^{(N-1)}(u, x) c_{j-i}(u-x) c_{m-j}(y-u) \\
& \leq \sum_{u, v} \sum_{i=0}^{m} p_{i}^{(N-1)}(u, v) a_{m-i}(u, v, x, y) \\
& =p_{m}^{(N)}(x, y) .
\end{aligned}
$$

This completes the proof.

Exercise 4.3. Convince yourself that (4.31) holds.

\subsubsection{Proof of (4.9)-(4.10)}

We prove two lemmas, and combine them with Proposition 4.2 to obtain (4.9)-(4.10).

We define the operators

$$
\begin{aligned}
\left(\mathcal{M}_{z} f\right)(x) & =H_{z}(x) f(x), \\
\left(\mathcal{H}_{z} f\right)(x) & =\left(H_{z} * f\right)(x), \\
\left(\mathcal{H}_{z}^{\prime} f\right)(x) & =\left(G_{z} * f\right)(x) .
\end{aligned}
$$


Lemma 4.4. For $N \geq 2$ and $z \geq 0$,

$$
\sum_{x} P_{z}^{(N)}(x, x+y)=\left[\left(\mathcal{H}_{z}^{\prime} \mathcal{M}_{z}\right)^{N-1} H_{z}\right](y) .
$$

Proof. The proof is by induction on $N$. For $N=2$, we conclude from (4.16) that

$$
\begin{aligned}
\sum_{x} P_{z}^{(2)}(x, x+y) & =\sum_{x} H_{z}(x)^{2} G_{z}(x+y) \\
& =\sum_{x} H_{z}(x)^{2} G_{z}(y-x)=\left[\left(\mathcal{H}_{z}^{\prime} \mathcal{M}_{z}\right) H_{z}\right](y)
\end{aligned}
$$

using $H_{z}(-x)=H_{z}(x)$ in the second step.

To advance the induction, we assume that (4.36) holds for $N-1$. By (4.20)-(4.21),

$$
\begin{aligned}
\sum_{x} P_{z}^{(N)}(x, x+y) & =\sum_{x, u, v} P_{z}^{(N-1)}(u, v) A_{z}(u, v, x, x+y) \\
& =\sum_{u, v} P_{z}^{(N-1)}(u, v) H_{z}(u-v) G_{z}(v-u+y) \\
& =\sum_{w}\left(\sum_{u} P_{z}^{(N-1)}(u, u+w)\right) H_{z}(-w) G_{z}(w+y) \\
& =\sum_{w}\left(\sum_{u} P_{z}^{(N-1)}(u, u+w)\right) H_{z}(w) G_{z}(y-w),
\end{aligned}
$$

where in the last step we replaced $w$ by $-w$ and used the fact that the first factor is unchanged by this replacement (see Exercise 4.5 below). Writing $F(w)$ for the first factor, the above is equal to

$$
\left(G_{z} * H_{z} F\right)(y)=\left(\mathcal{H}_{z}^{\prime} \mathcal{M}_{z} F\right)(y) .
$$

We then apply the induction hypothesis to complete the proof.

Exercise 4.5. Prove the identity $\sum_{u} P_{z}^{(N)}(u, u+w)=\sum_{u} P_{z}^{(N)}(u, u-w)$ used in (4.38).

The combination of Proposition 4.2 with Lemma 4.4 gives

$$
\sum_{x} \Pi_{z}^{(N)}(x) \leq \sum_{x} P_{z}^{(N)}(x, x)=\left[\left(\mathcal{H}_{z}^{\prime} \mathcal{M}_{z}\right)^{N-1} H_{z}\right](0) .
$$

Note that for $N=2$ the upper bound can be replaced by $\left[\left(\mathcal{H}_{z} \mathcal{M}_{z}\right)^{N-1} H_{z}\right](0)$, which is equal to the above right hand side in this special case. The right hand side of (4.40) can be estimated using the following lemma. 
Lemma 4.6. Given non-negative even functions $f_{0}, f_{1}, \ldots, f_{2 M}$ on $\mathbb{Z}^{d}$, define $\mathcal{H}_{j}$ and $\mathcal{M}_{j}$ to be respectively the operations of convolution with $f_{2 j}$ and multiplication by $f_{2 j-1}$, for $j=1, \ldots, M$. Then for any $k \in\{0, \ldots, 2 M\}$,

$$
\left\|\mathcal{H}_{M} \mathcal{M}_{M} \cdots \mathcal{H}_{1} \mathcal{M}_{1} f_{0}\right\|_{\infty} \leq\left\|f_{k}\right\|_{\infty} \prod\left\|f_{j} * f_{j^{\prime}}\right\|_{\infty}
$$

where the product is over disjoint consecutive pairs $j, j^{\prime}$ taken from the set $\{0, \ldots, 2 M\} \backslash\{k\}$ (e.g., for $k=3$ and $M=3$, the product has factors with $j, j^{\prime}$ equal to 0,$\left.1 ; 2,4 ; 5,6\right)$.

Proof. The proof is by induction on $M$. The desired result for $M=1$ is a consequence of the elementary estimates

$$
\sum_{y} f_{2}(x-y) f_{1}(y) f_{0}(y) \leq\left\{\begin{array}{l}
\left\|f_{0}\right\|_{\infty}\left\|f_{1} * f_{2}\right\|_{\infty} \\
\left\|f_{1}\right\|_{\infty}\left\|f_{0} * f_{2}\right\|_{\infty} \\
\left\|f_{2}\right\|_{\infty}\left\|f_{0} * f_{1}\right\|_{\infty},
\end{array}\right.
$$

where for the last of these inequalities we used the fact that $\sum_{y} f_{1}(y) f_{0}(y)=$ $\left(f_{0} * f_{1}\right)(0)$ for even $f_{0}$. To advance the induction, we assume that (4.41) holds for $1, \ldots, M-1$. We write the function inside the norm on the left hand side of (4.41) as $\mathcal{H}_{M} \mathcal{M}_{M} F_{M-1}$, with $F_{l}=\mathcal{H}_{l} \mathcal{M}_{l} \cdots \mathcal{H}_{1} \mathcal{M}_{1} f_{0}$, and estimate its infinity norm using the result for $M=1$. If we associate the infinity norm to $F_{M-1}$, an estimate of the form (4.41) follows from the induction hypothesis, for any $k \leq M-1$. It remains to show that the infinity norm can also be associated to $f_{2 M}$ or $f_{2 M-1}$.

We show this for the latter, and the former is similar. Applying the $M=1$ case to $\mathcal{H}_{M} \mathcal{M}_{M} F_{M-1}$ gives an upper bound $\left\|f_{2 M-1}\right\|_{\infty}\left\|f_{2 M} * F_{M-1}\right\|_{\infty}$. Let $\widetilde{\mathcal{H}}_{M-1}$ denote convolution by $f_{2 M} * f_{2 M-2}$, so that

$$
f_{2 M} * F_{M-1}=\widetilde{\mathcal{H}}_{M-1} \mathcal{M}_{M-1} F_{M-2}
$$

(with $F_{0}=f_{0}$ ). We apply the induction hypothesis to estimate the infinity norm of the right hand side, associating the infinity norm to $\widetilde{\mathcal{H}}_{M-1}$. This gives the desired estimate.

Exercise 4.7. Give the details omitted at the end of the above proof, for the case in which the infinity norm is associated to $f_{2 M}$.

Proof of (4.9)-(4.10). The bound (4.9) follows from (4.40) and Lemma 4.6.

It remains to prove (4.10). Fix $N \geq 2$. Our goal is to estimate

$$
\sum_{x}[1-\cos (k \cdot x)] \Pi_{z}^{(N)}(x)=\sum_{m=2}^{\infty} z^{m} \sum_{x}[1-\cos (k \cdot x)] \pi_{m}^{(N)}(x) .
$$

To do so, we investigate how the argument leading to (4.40) is modified by the factor $[1-\cos (k \cdot x)]$. 
Because of the factor $\prod_{i j \in L}\left(-U_{i j}\right)$ occurring in the definition of $\pi_{m}^{(N)}(x)$ (see (4.3)), a nonzero contribution occurs only for those $\omega$ for which $\omega(i)=$ $\omega(j)$ for each edge $i j \in L$. Let $I_{j}$ denote the $j^{\text {th }}$ time interval listed in (3.16) $(j=1, \ldots, 2 N-1)$, and let $y_{j}$ denote the displacement performed on $I_{j}$ by a walk $\omega$ contributing to $\pi_{m}^{(N)}(x)$. These displacements $y_{j}$ correspond to the subwalk displacements in Figures 3.4 and 4.2. The constraints that $\omega(i)=\omega(j)$ for all ij $\in L$, together with the subinterval structure (3.16), impose the constraints

$$
y_{1}+y_{2}=0, \quad \sum_{j=2 p}^{2 p+2} y_{j}=0 \quad(p=1, \ldots, N-2), \quad y_{2 N-2}+y_{2 N-1}=0 .
$$

It can also be seen from this (see also Fig. 3.4) that the total displacement $x$ is given by

$$
x=\sum_{i=1}^{\lfloor N / 2\rfloor} y_{4 i-1}=\sum_{i=1}^{\lceil N / 2\rceil} y_{4 i-3}=-\sum_{i=1}^{N-1} y_{2 i}
$$

(we need only the first equality).

Let $t=\sum_{j=1}^{J} t_{j}$. Taking the real part of the telescoping sum

$$
1-\mathrm{e}^{\mathrm{i} t}=\sum_{j=1}^{J}\left[1-\mathrm{e}^{\mathrm{i} t_{j}}\right] \mathrm{e}^{\mathrm{i} \sum_{m=1}^{j-1} t_{m}}
$$

leads to the bound

$$
1-\cos t \leq \sum_{j=1}^{J}\left[1-\cos t_{j}\right]+\sum_{j=1}^{J} \sin t_{j} \sin \left(\sum_{m=1}^{j-1} t_{m}\right) .
$$

It is a consequence of the identity $\sin (x+y)=\sin x \cos y+\cos x \sin y$ that $|\sin (x+y)| \leq|\sin x|+|\sin y|$. Applying this recursively gives

$$
1-\cos t \leq \sum_{j=1}^{J}\left[1-\cos t_{j}\right]+\sum_{j=1}^{J} \sum_{m=1}^{j-1}\left|\sin t_{j}\right|\left|\sin t_{m}\right| .
$$

In the last term we use $|a b| \leq\left(a^{2}+b^{2}\right) / 2$, and then $1-\cos ^{2} a \leq 2[1-\cos a]$, to obtain 


$$
\begin{aligned}
1-\cos t & \leq \sum_{j=1}^{J}\left[1-\cos t_{j}\right]+\frac{1}{2} \sum_{j=1}^{J} \sum_{m=1}^{j-1}\left[\sin ^{2} t_{j}+\sin ^{2} t_{m}\right] \\
& \leq \sum_{j=1}^{J}\left[1-\cos t_{j}\right]+J \sum_{j=1}^{J} \sin ^{2} t_{j} \\
& =\sum_{j=1}^{J}\left[1-\cos t_{j}\right]+J \sum_{j=1}^{J}\left[1-\cos ^{2} t_{j}\right] \\
& \leq(2 J+1) \sum_{j=1}^{J}\left[1-\cos t_{j}\right] .
\end{aligned}
$$

We use the decomposition of $x$ given by the first equality of (4.46), and apply (4.50) with $t=k \cdot x=\sum_{j=1}^{\lfloor N / 2\rfloor} k \cdot y_{4 j-1}$, to obtain

$$
1-\cos (k \cdot x) \leq(N+1) \sum_{j=1}^{\lfloor N / 2\rfloor}\left[1-\cos \left(k \cdot y_{4 j-1}\right)\right] .
$$

The modification of the upper bound (4.40) due to the factor $\left[1-\cos \left(k \cdot y_{4 j-1}\right)\right]$ is simply to replace one of the factors $H_{z}$ or $G_{z}$ occurring in the right hand side by $\left[1-\cos \left(k \cdot y_{4 j-1}\right)\right] H_{z}\left(y_{4 j-1}\right)$. Then we apply Lemma 4.6 , associating the infinity norm to this particular factor, to obtain the desired estimate (4.10).

\section{Convergence for the Self-Avoiding Walk}

In this section, we prove convergence of the lace expansion for the nearestneighbour model in sufficiently high dimensions, and for sufficiently spread-out models in dimensions $d>4$. As part of the proof, we will show that the critical bubble diagram $B\left(z_{c}\right)$ is finite in these cases, and hence, by Theorem 2.3 , that the critical exponent $\gamma$ exists and equals 1 . This is restated in the following theorem.

Theorem 5.1. The bubble condition $B\left(z_{c}\right)<\infty$ for the self-avoiding walk holds for the nearest-neighbour model in dimensions $d \geq d_{0}$, and for the spread-out model with $L \geq L_{0}(d)$ in dimensions $d>4$, for some constants $d_{0}$ and $L_{0}(d)$. Thus the critical exponent $\gamma$ exists and equals 1 , in the sense that $\chi(z) \simeq\left(1-z / z_{c}\right)^{-1}$ as $z \rightarrow z_{c}^{-}$.

Remark 5.2. The conclusion of Theorem 5.1 can easily be improved to an asymptotic formula $\chi(z) \sim A\left(1-z / z_{c}\right)^{-1}$ as $z \rightarrow z_{c}^{-}$. See Exercise 5.19 below. 
Recall that $H_{z}(x)=G_{z}(x)-\delta_{0, x}=\sum_{n=1}^{\infty} c_{n}(x) z^{n}$, so that $\left\|H_{z}\right\|_{2}^{2}=$ $\left\|G_{z}\right\|_{2}^{2}-1=B(z)-1$. We will prove not just that the critical bubble diagram $B\left(z_{c}\right)$ is finite, but that in fact $\left\|H_{z_{c}}\right\|_{2}^{2}=B\left(z_{c}\right)-1$ is small, under the hypotheses of Theorem 5.1. As a preliminary, we first analyze some related issues for simple random walk.

\subsection{Random-Walk Estimates}

By the Parseval relation, $\left\|H_{z_{c}}\right\|_{2}^{2}=\left\|\hat{H}_{z_{c}}\right\|_{2}^{2}=\left\|\hat{G}_{z_{c}}-1\right\|_{2}^{2}$. By (1.18), the random walk analogue of the latter is the integral

$$
\int_{[-\pi, \pi]^{d}}\left|\frac{1}{[1-\hat{D}(k)]}-1\right|^{2} \frac{\mathrm{d}^{d} k}{(2 \pi)^{d}}=\int_{[-\pi, \pi]^{d}} \frac{\hat{D}(k)^{2}}{[1-\hat{D}(k)]^{2}} \frac{\mathrm{d}^{d} k}{(2 \pi)^{d}} .
$$

The following proposition shows that this integral is small under the hypotheses of Theorem 5.1. (We have already encountered a closely related integral in Exercise 1.7.)

Proposition 5.3. Let $d>4$. Then

$$
\int_{[-\pi, \pi]^{d}} \frac{\hat{D}(k)^{2}}{[1-\hat{D}(k)]^{2}} \frac{\mathrm{d}^{d} k}{(2 \pi)^{d}} \leq \beta,
$$

with $\beta=K(d-4)^{-1}$ ( $K$ a universal constant) for the nearest-neighbour model, and with $\beta=K L^{-d}$ ( $K$ dependent on $d$ ) for the spread-out model.

Proof. This is a calculus problem. For the nearest-neighbour model, a proof can be found in [158, Lemma A.3]. For the spread-out model, there is a proof in [158, Lemma A.5] but with a $\beta$ which is larger by a factor $(\log L)^{d / 2}$. We show here how the improvement can be achieved for the spread-out model.

It is shown in [120] that there are positive constants $\eta, c_{1}$ (independent of $L)$ such that for all $k \in[-\pi, \pi]^{d}$,

$$
\begin{aligned}
& 1-\hat{D}(k) \geq c_{1} L^{2}|k|^{2} \quad\left(\|k\|_{\infty} \leq L^{-1}\right), \\
& 1-\hat{D}(k)>\eta \quad\left(\|k\|_{\infty} \geq L^{-1}\right) .
\end{aligned}
$$

The integral $(2 \pi)^{-d} \int_{[-\pi, \pi]^{d}} \hat{D}(k)^{2} \mathrm{~d}^{d} k$ is equal to $(D * D)(0)$, which is the probability of return to the origin after two steps, namely $|\Omega|^{-1}$. For $j \geq 4$ even, it follows from (5.3)-(5.4) that

$$
\begin{aligned}
\int_{[-\pi, \pi]^{d}} \hat{D}(k)^{j} \frac{\mathrm{d}^{d} k}{(2 \pi)^{d}} \leq & \int_{\mathbb{R}^{d}} \mathrm{e}^{-c_{1} j L^{2}|k|^{2}} \frac{\mathrm{d}^{d} k}{(2 \pi)^{d}} \\
& \quad+\int_{[-\pi, \pi]^{d}} \hat{D}(k)^{2}(1-\eta)^{j-2} \frac{\mathrm{d}^{d} k}{(2 \pi)^{d}} \\
\leq & \operatorname{const} L^{-d} j^{-d / 2}+|\Omega|^{-1}(1-\eta)^{j-2} \\
\leq & \operatorname{const} L^{-d} j^{-d / 2} .
\end{aligned}
$$


For $j \geq 3$ odd,

$$
\begin{aligned}
(2 \pi)^{-d} \int_{[-\pi, \pi]^{d}}|\hat{D}(k)|^{j} \mathrm{~d}^{d} k & \leq(2 \pi)^{-d} \int_{[-\pi, \pi]^{d}} \hat{D}(k)^{j-1} \mathrm{~d}^{d} k \\
& \leq \operatorname{const} L^{-d} j^{-d / 2},
\end{aligned}
$$

applying the estimate for $j$ even in the last step. Now we expand $[1-\hat{D}(k)]^{-2}$ in (5.2) as $\sum_{j=1}^{\infty} j \hat{D}(k)^{j-1}$, and use the above estimates to see that the left hand side of (5.2) is bounded above by a multiple of $L^{-d}$, assuming $d>4$.

Exercise 5.4. Prove (5.2) for the nearest-neighbour model, with $\beta=K(d-$ $4)^{-1}$.

The following lemma notes some useful implications of (5.2). The left hand sides of (5.8)-(5.9) are respectively the random walk analogues of $\left\|G_{z_{c}}\right\|_{2}^{2}$ and of $\left\|[1-\cos (k \cdot x)] G_{z_{c}}(x)\right\|_{\infty}=\left\|[1-\cos (k \cdot x)] H_{z_{c}}(x)\right\|_{\infty}$ (cf. (4.10)).

Lemma 5.5. If (5.2) holds, then for $z \in[0,1 /|\Omega|]$,

$$
\begin{aligned}
\sup _{x \in \mathbb{Z}^{d}} D(x) & \leq \beta, \\
\left\|C_{z}\right\|_{2}^{2} & \leq 1+3 \beta, \\
\left\|[1-\cos (k \cdot x)] C_{z}(x)\right\|_{\infty} & \leq 5(1+3 \beta)[1-\hat{D}(k)] .
\end{aligned}
$$

We first prove (5.7)-(5.8).

Proof of (5.7)-(5.8). The left hand side of (5.7) is simply $|\Omega|^{-1}$. Since the left hand side of $(5.2)$ is at least $(2 \pi)^{-d} \int_{[-\pi, \pi]^{d}} \hat{D}(k)^{2} \mathrm{~d}^{d} k=|\Omega|^{-1}$, the bound (5.7) follows.

For (5.8) (and also (5.9)), it suffices to consider $z=1 /|\Omega|$. We use the Parseval relation to rewrite the left hand side as $\left\|\hat{C}_{1 /|\Omega|}\right\|_{2}^{2}$. By (1.18), this equals

$$
\begin{aligned}
& \int_{[-\pi, \pi]^{d}} \frac{1}{[1-\hat{D}(k)]^{2}} \frac{\mathrm{d}^{d} k}{(2 \pi)^{d}} \\
& \quad=\int_{[-\pi, \pi]^{d}}\left(1+2 \frac{\hat{D}(k)}{[1-\hat{D}(k)]}+\frac{\hat{D}(k)^{2}}{[1-\hat{D}(k)]^{2}}\right) \frac{\mathrm{d}^{d} k}{(2 \pi)^{d}} \\
& \quad \leq \int_{[-\pi, \pi]^{d}}\left(1+3 \frac{\hat{D}(k)^{2}}{[1-\hat{D}(k)]^{2}}\right) \frac{\mathrm{d}^{d} k}{(2 \pi)^{d}},
\end{aligned}
$$

by Exercise 5.6. The right hand side is at most $1+3 \beta$, assuming (5.2).

Exercise 5.6. Prove the inequality (5.10) by comparing the integrals of $\hat{D}[1-$ $\hat{D}]^{-1}$ and $\hat{D}^{2}[1-\hat{D}]^{-2}$. 
Before proving (5.9), we develop some useful preliminaries. We first note that

$$
\sum_{x} \cos (k \cdot x) C_{z}(x) \mathrm{e}^{\mathrm{i} l \cdot x}=\frac{1}{2}\left[\hat{C}_{z}(l+k)+\hat{C}_{z}(l-k)\right] .
$$

Therefore, applying the general fact that $\|f\|_{\infty} \leq\|\hat{f}\|_{1}$, we obtain

$$
\left\|[1-\cos (k \cdot x)] C_{z}(x)\right\|_{\infty} \leq\left\|\hat{C}_{z}(l)-\frac{1}{2}\left[\hat{C}_{z}(l+k)+\hat{C}_{z}(l-k)\right]\right\|_{1},
$$

where the $L^{1}$ norm involves integration with respect to $l$, with $k$ fixed. The expression $\hat{C}_{z}(l)-\frac{1}{2}\left(\hat{C}_{z}(l+k)+\hat{C}_{z}(l-k)\right)$ is closely related to a sort of second derivative of $\hat{C}_{z}(l)$, and in general we make the abbreviation

$$
-\frac{1}{2} \Delta_{k} \hat{A}(l)=\hat{A}(l)-\frac{1}{2}(\hat{A}(l+k)+\hat{A}(l-k)) .
$$

In this notation, (5.12) reads

$$
\|\left[1-\cos (k \cdot x) C_{z}(x)\left\|_{\infty} \leq \frac{1}{2}\right\| \Delta_{k} \hat{C}_{z}(l) \|_{1},\right.
$$

where the integration on the right hand side is with respect to $l$.

Lemma 5.7. Suppose that $a(-x)=a(x)$ for all $x \in \mathbb{Z}^{d}$, and let

$$
\hat{A}(k)=\frac{1}{1-\hat{a}(k)} .
$$

Then for all $k, l \in[-\pi, \pi]^{d}$,

$$
\begin{aligned}
\frac{1}{2}\left|\Delta_{k} \hat{A}(l)\right| \leq & \frac{1}{2}[\hat{A}(l-k)+\hat{A}(l+k)] \hat{A}(l)\left[\hat{a}^{\mathrm{av}}(0)-\hat{a}^{\mathrm{av}}(k)\right] \\
& +4 \hat{A}(l-k) \hat{A}(l) \hat{A}(l+k)\left[\hat{a}^{\mathrm{av}}(0)-\hat{a}^{\mathrm{av}}(k)\right]\left[\hat{a}^{\mathrm{av}}(0)-\hat{a}^{\mathrm{av}}(l)\right],
\end{aligned}
$$

where $a^{\text {av }}(x)=|a(x)|$.

Proof of (5.9). We use Lemma 5.7 to estimate the right hand side of (5.14), with $\hat{a}(k)=\hat{D}(k)$ and $\hat{A}(k)=\hat{C}_{1 /|\Omega|}(k)$. Writing the latter simply as $\hat{C}(k)$, this gives

$$
\frac{1}{2}\left|\Delta_{k} \hat{C}(l)\right| \leq[1-\hat{D}(k)]\left(\frac{1}{2}[\hat{C}(l-k)+\hat{C}(l+k)] \hat{C}(l)+4 \hat{C}(l-k) \hat{C}(l+k)\right) .
$$

Therefore, by the Cauchy-Schwarz inequality,

$$
\frac{1}{2}\left\|\Delta_{k} \hat{C}\right\|_{1} \leq[1-\hat{D}(k)] 5\|\hat{C}\|_{2}^{2},
$$

and (5.9) follows from (5.8). 
Proof of Lemma 5.7. Since $a$ is even, $\hat{a}(l)=\sum_{x} a(x) \cos (l \cdot x)$. For such an $a$, we define

$$
\begin{aligned}
& \hat{a}^{\cos }(l, k)=\sum_{x} a(x) \cos (l \cdot x) \cos (k \cdot x)=\frac{1}{2}[\hat{a}(l-k)+\hat{a}(l+k)], \\
& \hat{a}^{\sin }(l, k)=\sum_{x} a(x) \sin (l \cdot x) \sin (k \cdot x)=\frac{1}{2}[\hat{a}(l-k)-\hat{a}(l+k)] .
\end{aligned}
$$

We first show that, for all $k, l \in[-\pi, \pi]^{d}$,

$$
\begin{aligned}
-\frac{1}{2} \Delta_{k} \hat{A}(l)= & \frac{1}{2}[\hat{A}(l-k)+\hat{A}(l+k)] \hat{A}(l)\left[\hat{a}(l)-\hat{a}^{\cos }(l, k)\right] \\
& -\hat{A}(l-k) \hat{A}(l) \hat{A}(l+k) \hat{a}^{\sin }(l, k)^{2} .
\end{aligned}
$$

Let $\hat{a}_{ \pm}=\hat{a}(l \pm k)$ and write $\hat{a}=\hat{a}(l)$. Direct computation using (5.13) gives

$$
\begin{aligned}
- & \frac{1}{2} \Delta_{k} \hat{A}(l) \\
\quad & \frac{1}{2} \hat{A}(l) \hat{A}(l+k) \hat{A}(l-k)\left[\left[2 \hat{a}-\hat{a}_{+}-\hat{a}_{-}\right]+\left[2 \hat{a}_{+} \hat{a}_{-}-\hat{a} \hat{a}_{-}-\hat{a} \hat{a}_{+}\right]\right] \\
& =\hat{A}(l) \hat{A}(l+k) \hat{A}(l-k)\left[\left[\hat{a}(l)-\hat{a}^{\cos }(l, k)\right]+\left[\hat{a}_{+} \hat{a}_{-}-\hat{a}(l) \hat{a}^{\cos }(l, k)\right]\right]
\end{aligned}
$$

using (5.19) in the last step. By definition, and using the identity $\cos (u+v)=$ $\cos u \cos v-\sin u \sin v$,

$$
\begin{aligned}
\hat{a}_{+} \hat{a}_{-} & =\sum_{x, y} a(x) a(y) \cos ((l+k) \cdot x) \cos ((l-k) \cdot y) \\
& =\hat{a}^{\cos }(l, k)^{2}-\hat{a}^{\sin }(l, k)^{2} .
\end{aligned}
$$

Substitution of (5.23) in (5.22) gives

$$
-\frac{1}{2} \Delta_{k} \hat{A}(l)=\hat{A}(l-k) \hat{A}(l) \hat{A}(l+k)\left[\left[\hat{a}(l)-\hat{a}^{\cos }(l, k)\right]\left[1-\hat{a}^{\cos }(l, k)\right]-\hat{a}^{\sin }(l, k)^{2}\right] .
$$

Finally, we use (5.19) to rewrite $1-\hat{a}^{\cos }(l, k)$ and obtain (5.21).

Now we use (5.21) to prove (5.16). First, we note that

$$
\begin{aligned}
\left|\hat{a}(l)-\hat{a}^{\cos }(l, k)\right| & \leq \sum_{x}[1-\cos (k \cdot x)]|\cos (l \cdot x)||a(x)| \\
& \leq \hat{a}^{\mathrm{av}}(0)-\hat{a}^{\mathrm{av}}(k) .
\end{aligned}
$$

Also, by (5.20) and the Cauchy-Schwarz inequality,

$$
\hat{a}^{\sin }(k, l)^{2} \leq\left(\sum_{x}|a(x)| \sin ^{2}(k \cdot x)\right)\left(\sum_{x}|a(x)| \sin ^{2}(l \cdot x)\right) .
$$


With the elementary estimate $\sin ^{2} t=1-\cos ^{2} t \leq 2[1-\cos t]$, this gives

$$
\begin{aligned}
\hat{a}^{\sin }(k, l)^{2} & \leq \sum_{x}|a(x)|\left[1-\cos ^{2}(k \cdot x)\right] \sum_{y}|a(y)|\left[1-\cos ^{2}(l \cdot y)\right] \\
& \leq 4\left[\hat{a}^{\mathrm{av}}(0)-\hat{a}^{\mathrm{av}}(k)\right]\left[\hat{a}^{\mathrm{av}}(0)-\hat{a}^{\mathrm{av}}(l)\right] .
\end{aligned}
$$

The desired estimate (5.16) then follows from (5.21), (5.25) and (5.27).

\subsection{Convergence of the Expansion}

In this section we prove convergence of the lace expansion, assuming (5.2), and also prove Theorem 5.1. Convergence will be proved in the process of proving the following theorem, which shows that if the critical simple random walk bubble diagram is sufficiently small, then the critical self-avoiding walk bubble diagram is also small. (In both diagrams, the trivial term 1 is omitted to obtain a small quantity.)

Theorem 5.8. There is $a \beta_{0}>0$ and a constant $c$ such that if (5.2) holds with $\beta \leq \beta_{0}$, then $B\left(z_{c}\right)-1$ is less than $c \beta$.

Proof of Theorem 5.1. This is an immediate consequence of Proposition 5.3, Theorem 5.8, and Theorem 2.3.

We will prove Theorem 5.8 in the remainder of Section 5 . The proof is inspired by the method of [32]. It is possible to go beyond Theorem 5.8 in several respects, and this will be discussed in Section 6. In particular, critical exponents of the nearest-neighbour strictly self-avoiding walk in dimensions $d \geq 5$ are computed in [97, 98].

It is not obvious, at first, how to approach the issue of convergence of the lace expansion. The conclusion of Theorem 5.8 is that $\left\|H_{z_{c}}\right\|_{2}$ is small. On the other hand, recall from (4.11) that

$$
\left\|H_{z} * G_{z}\right\|_{\infty} \leq\left\|H_{z}\right\|_{\infty}+\left\|H_{z}\right\|_{2}^{2} .
$$

To use this to perform the sum over $N$ in Theorem 4.1 to estimate $\Pi_{z}$, we already need to know that $\left\|H_{z}\right\|_{2}^{2}$ is small uniformly in $z<z_{c}$. The following elementary lemma will be used to allow us to pick ourselves up by our bootstraps.

Lemma 5.9. Let $a<b$, let $f$ be a continuous function on the interval $\left[z_{1}, z_{2}\right)$, and assume that $f\left(z_{1}\right) \leq a$. Suppose for each $z \in\left(z_{1}, z_{2}\right)$ that if $f(z) \leq b$ then in fact $f(z) \leq a$. Then $f(z) \leq a$ for all $z \in\left[z_{1}, z_{2}\right)$.

Proof. By hypothesis, $f(z)$ cannot lie strictly between $a$ and $b$ for any $z \in$ $\left(z_{1}, z_{2}\right)$. Since $f\left(z_{1}\right) \leq a$, it follows by continuity that $f(z) \leq a$ for all $z \in$ $\left[z_{1}, z_{2}\right)$.

For $z \in\left[0, z_{c}\right)$, we define $p(z) \in[0,1 /|\Omega|)$ by 


$$
\hat{G}_{z}(0)=\chi(z)=\frac{1}{1-p(z)|\Omega|}=\hat{C}_{p(z)}(0),
$$

which is equivalent to

$$
p(z)|\Omega|=1-\frac{1}{\chi(z)} .
$$

Our choice of $f$ is motivated, in part, by the intuition that $\hat{G}_{z}(k)$ and $\hat{C}_{p(z)}(k)$ are comparable in size. We also expect $\frac{1}{2} \Delta_{k} \hat{G}_{z}(l)$ and $\frac{1}{2} \Delta_{k} \hat{C}_{p(z)}(l)$ to be comparable. However, rather than comparing the latter directly, we will compare $\frac{1}{2} \Delta_{k} \hat{G}_{z}(l)$ with

$$
\begin{gathered}
U_{p(z)}(k, l)=16 \hat{C}_{p(z)}(k)^{-1}\left(\hat{C}_{p(z)}(l-k) \hat{C}_{p(z)}(l)+\hat{C}_{p(z)}(l+k) \hat{C}_{p(z)}(l)\right. \\
\left.+\hat{C}_{p(z)}(l-k) \hat{C}_{p(z)}(l+k)\right)
\end{gathered}
$$

which can be seen using (5.16) to be an upper bound for $\frac{1}{2}\left|\Delta_{k} \hat{C}_{p(z)}(l)\right|$.

We will apply Lemma 5.9 with $z_{1}=0, z_{2}=z_{c}, b=4, a=1+\operatorname{const} \beta$ (the constant being determined in the course of the proof), and

$$
f(z)=\max \left\{f_{1}(z), f_{2}(z), f_{3}(z)\right\}
$$

where

$$
\begin{gathered}
f_{1}(z)=z|\Omega|, \quad f_{2}(z)=\sup _{k \in[-\pi, \pi]^{d}} \frac{\left|\hat{G}_{z}(k)\right|}{\hat{C}_{p(z)}(k)}, \\
f_{3}(z)=\sup _{k, l \in[-\pi, \pi]^{d}} \frac{\frac{1}{2}\left|\Delta_{k} \hat{G}_{z}(l)\right|}{U_{p(z)}(k, l)} .
\end{gathered}
$$

Note that the factor $\hat{C}_{p(z)}(k)^{-1}$ in the denominator of $f_{3}$ becomes arbitrarily small when $k=0$ and $z \rightarrow z_{c}^{-}$. We will verify in Lemmas $5.12,5.14$ and 5.16 that the hypotheses of Lemma 5.9 hold when $\beta$ is sufficiently small. From this, we can conclude that $f(z) \leq a=1+\operatorname{const} \beta$ uniformly in $z \in\left[0, z_{c}\right)$.

Proof of Theorem 5.8. We will show below in Lemma 5.10 that it follows from $f(z) \leq a$ (which we will conclude as noted above) that $\left\|H_{z}\right\|_{2}^{2} \leq c_{a} \beta$, where $c_{a}$ is the constant of Lemma 5.10 when $f(z) \leq K=a$. This proves $\left\|H_{z}\right\|_{2}^{2} \leq c_{a} \beta$ uniformly in $z<z_{c}$. By the monotone convergence theorem, this implies that

$$
\left\|H_{z_{c}}\right\|_{2}^{2}=\lim _{z \rightarrow z_{c}^{-}}\left\|H_{z}\right\|_{2}^{2} \leq c_{a} \beta
$$

which proves Theorem 5.8 since $B\left(z_{c}\right)-1=\left\|H_{z_{c}}\right\|_{2}^{2}$ by $(4.12)$.

Note that the inequality $f_{2}(z) \leq a$ implies the infrared bound

$$
\hat{G}_{z}(k) \leq a \hat{C}_{p(z)}(k)
$$

(we will actually prove in $(5.53)-(5.60)$ that $\hat{G}_{z}(k) / \hat{C}_{p(z)}(k)=1+O(\beta)$, which implies, in particular, that $\hat{G}_{z}(k) \geq 0$, permitting removal of the absolute value on the left hand side of (5.36)). 
Before going into the details, the basic strategy is as follows. First, it is straightforward to verify the two hypotheses on $f$ in Lemma 5.9 that $f$ is continuous and that $f(0) \leq a$, and the main work goes into verifying that $f(z) \leq b$ implies that $f(z) \leq a$. For this, we use the assumption $f(z) \leq b$ to compare norms of $H_{z}$ with norms of $C_{p(z)}$, and use (5.2) and Lemma 5.5 to see that the latter are small. We then apply Theorem 4.1 to conclude that $\hat{\Pi}_{z}(k)$ is as small as we like, assuming that $\beta$ is sufficiently small. Importantly, this can be done even for a poor (large) value of $b$, because the effect of taking $\beta$ small compensates for the lack of sharpness in the bound $f(z) \leq b$. This implies that $G_{z}(k)$ is close to a simple random walk quantity, and from this we will be able to conclude the sharper bound $f(z) \leq a$. The details are carried out below.

Lemma 5.10. Fix $z \in\left(0, z_{c}\right)$, assume that $f$ of (5.32) obeys $f(z) \leq K$, and assume (5.2). Then there is a constant $c_{K}$, independent of $z$, such that

$$
\begin{gathered}
\left\|[1-\cos (k \cdot x)] H_{z}\right\|_{\infty} \leq c_{K}(1+\beta) \hat{C}_{p(z)}(k)^{-1}, \\
\left\|H_{z}\right\|_{2}^{2} \leq c_{K} \beta, \quad\left\|H_{z}\right\|_{\infty} \leq c_{K} \beta .
\end{gathered}
$$

Proof. As in (5.14),

$$
\begin{aligned}
\left\|[1-\cos (k \cdot x)] H_{z}\right\|_{\infty} & =\left\|[1-\cos (k \cdot x)] G_{z}\right\|_{\infty} \\
& \leq \frac{1}{2}\left\|\Delta_{k} \hat{G}_{z}\right\|_{1} .
\end{aligned}
$$

It then follows from $f_{3}(z) \leq K$, the Cauchy-Schwarz inequality, and (5.8) that

$$
\begin{aligned}
\left\|[1-\cos (k \cdot x)] H_{z}\right\|_{\infty} & \leq 16 K \hat{C}_{p(z)}(k)^{-1} 3\left\|\hat{C}_{p(z)}\right\|_{2}^{2} \\
& \leq 48(1+3 \beta) K \hat{C}_{p(z)}(k)^{-1}
\end{aligned}
$$

which gives (5.37).

Next, we estimate $\left\|H_{z}\right\|_{2}^{2}$. We first use subadditivity and $f_{1}(z) \leq K$ to obtain

$$
H_{z}(x) \leq z|\Omega|\left(D * G_{z}\right)(x) \leq K\left(D * G_{z}\right)(x) .
$$

Using $f_{2}(z) \leq K$, the Parseval relation, and (5.2), this implies that

$$
\begin{aligned}
\left\|H_{z}\right\|_{2}^{2} & \leq K^{2}\left\|D * G_{z}\right\|_{2}^{2}=K^{2}\left\|\hat{D} \hat{G}_{z}\right\|_{2}^{2} \\
& \leq K^{4}\left\|\hat{D} \hat{C}_{p(z)}\right\|_{2}^{2}=K^{4}\left\|D * C_{p(z)}\right\|_{2}^{2} \\
& \leq K^{4}\left\|D * C_{1 /|\Omega|}\right\|_{2}^{2}=K^{4}\left\|\hat{D}[1-\hat{D}]^{-1}\right\|_{2}^{2} \leq K^{4} \beta .
\end{aligned}
$$

This proves the first bound of (5.38).

Iteration of (5.41) gives $H_{z}(x) \leq K D(x)+K^{2}\left(D * D * G_{z}\right)(x)$. Therefore, 


$$
\begin{aligned}
\left\|H_{z}\right\|_{\infty} & \leq K\|D\|_{\infty}+K^{2}\left\|\hat{D}^{2} \hat{G}_{z}\right\|_{1} \\
& \leq K \beta+K^{3}\left\|\hat{D}^{2} \hat{C}_{p(z)}\right\|_{1} \\
& =K \beta+K^{3}\left(D * D * C_{p(z)}\right)(0) \\
& \leq K \beta+K^{3}\left(D * D * C_{p(z)} * C_{p(z)}\right)(0) \\
& \leq K \beta+K^{3}\left(D * D * C_{1 /|\Omega|} * C_{1 /|\Omega|}\right)(0) \\
& \leq K \beta+K^{3} \beta,
\end{aligned}
$$

using (5.7) in the second inequality, and the inverse Fourier transform and (5.2) in the last.

Remark. The bounds of Lemma 5.10 can be combined with Theorem 4.1 to give bounds on $\Pi^{(N)}$, and hence on $\Pi$. This is the content of the following lemma. Note that once we have verified that the hypotheses of Lemma 5.9 all hold, we can conclude that $f(z) \leq a=1+\operatorname{const} \beta$, thereby verifying the hypothesis $f(z) \leq K$ of Lemma 5.11 with $K=a$. After the fact, this then gives unconditional bounds on $\Pi_{z}$ (of course assuming (5.2)). These bounds are then of lasting importance (see, e.g., Exercises 5.17-5.18).

Lemma 5.11. Fix $z \in\left(0, z_{c}\right)$, assume that $f$ of (5.32) obeys $f(z) \leq K$, and assume that (5.2) holds. There is a constant $\bar{c}_{K}$, independent of $z$, such that if $\beta$ is sufficiently small (independent of $z$ ), then

$$
\begin{gathered}
\sum_{x \in \mathbb{Z}^{d}}\left|\Pi_{z}(x)\right| \leq \bar{c}_{K} \beta \\
\sum_{x \in \mathbb{Z}^{d}}[1-\cos (k \cdot x)]\left|\Pi_{z}(x)\right| \leq \bar{c}_{K} \beta \hat{C}_{p(z)}(k)^{-1} .
\end{gathered}
$$

Proof. It follows from Theorem 4.1, Lemma 5.10, and the estimate (5.28) that there is a constant $c_{K}^{\prime}$ such that

$$
\sum_{x \in \mathbb{Z}^{d}} \Pi_{z}^{(N)}(x) \leq\left(c_{K}^{\prime} \beta\right)^{N}
$$

for all $N \geq 1$, and

$$
\sum_{x \in \mathbb{Z}^{d}}[1-\cos (k \cdot x)] \Pi_{z}^{(N)}(x)\left\{\begin{array}{lr}
=0 & \text { if } N=1 \\
\leq \hat{C}_{p(z)}(k)^{-1} N^{2}\left(c_{K}^{\prime} \beta\right)^{N-1} & \text { if } N \geq 2 .
\end{array}\right.
$$

The bounds (5.44)-(5.45) then follow immediately.

We now confirm that $f$ of (5.32) obeys the hypotheses of Lemma 5.9, with $z_{1}=0, z_{2}=z_{c}, b=4$ and $a=1+\operatorname{const} \beta$ (the particular value 4 for $b$ is not an essential choice). We first verify that $f(0)=1$, which is of course less than $a$.

Lemma 5.12. The function $f$ of (5.32) obeys $f(0)=1$. 
Proof. By definition, $f_{1}(0)=0$. Also, $p(0)=0$ by $(5.30)$ and hence $f_{2}(0)=1$. Finally, $f_{3}(0)=0$.

To prove the continuity of $f$, we will use the following elementary lemma.

Lemma 5.13. Let $\left(f_{\alpha}\right)_{\alpha \in A}$ be an equicontinuous family of functions on an interval $\left[t_{1}, t_{2}\right]$, and suppose that $\sup _{\alpha \in A} f_{\alpha}(t)<\infty$ for each $t \in\left[t_{1}, t_{2}\right]$. Then $\sup _{\alpha \in A} f_{\alpha}$ is continuous on $\left[t_{1}, t_{2}\right]$.

Proof. Let $\bar{f}=\sup _{\alpha \in A} f_{\alpha}$, and let $\epsilon>0$ be given. The statement that $\left(f_{\alpha}\right)_{\alpha \in A}$ is equicontinuous means that there is a $\delta>0$ such that $\left|f_{\alpha}(s)-f_{\alpha}(t)\right|<\epsilon / 2$ whenever $|s-t|<\delta$, uniformly in $\alpha \in A$. Fix $s, t$ with $|s-t|<\delta$, and assume without loss of generality that $\bar{f}(s) \geq \bar{f}(t)$. Choose $\alpha^{\prime}$ such that $0 \leq$ $\bar{f}(s)-f_{\alpha^{\prime}}(s)<\epsilon / 2$. Then

$$
\begin{aligned}
0 & \leq \bar{f}(s)-\bar{f}(t) \leq \bar{f}(s)-f_{\alpha^{\prime}}(t) \\
& \leq\left[\bar{f}(s)-f_{\alpha^{\prime}}(s)\right]+\left|f_{\alpha^{\prime}}(s)-f_{\alpha^{\prime}}(t)\right| \\
& <\frac{\epsilon}{2}+\frac{\epsilon}{2}=\epsilon .
\end{aligned}
$$

Therefore, $\bar{f}$ is continuous.

Lemma 5.14. The function $f$ of (5.32) is continuous on the interval $\left[0, z_{c}\right)$.

Proof. It suffices to show that each of $f_{1}, f_{2}, f_{3}$ is continuous on $\left[0, z_{c}\right)$. The function $f_{1}$ is linear, so it is certainly continuous.

For $f_{2}$, it suffices to show that $f_{2}$ is continuous in $[0, r]$ for every $r<z_{c}$. By Lemma 5.13 , it suffices to show that $\left|\hat{G}_{z}(k)\right| / \hat{C}_{p(z)}(k)$ is equicontinuous in $z \in[0, r]$. Here $\alpha$ is $k$. Since $\left(\left|f_{\alpha}\right|\right)_{\alpha \in A}$ is an equicontinuous family whenever $\left(f_{\alpha}\right)_{\alpha \in A}$ is, it suffices to obtain a bound on the derivative

$$
\frac{\mathrm{d}}{\mathrm{d} z} \frac{\hat{G}_{z}(k)}{\hat{C}_{p(z)}(k)}=\frac{1}{\hat{C}_{p(z)}(k)^{2}}\left[\hat{C}_{p(z)}(k) \frac{\mathrm{d} \hat{G}_{z}(k)}{\mathrm{d} z}-\left.\hat{G}_{z}(k) \frac{\mathrm{d} \hat{C}_{p}(k)}{\mathrm{d} p}\right|_{p=p(z)} \frac{\mathrm{d} p(z)}{\mathrm{d} z}\right],
$$

uniformly in $k$ and in $z \in[0, r]$. This follows from the bounds

$$
\begin{aligned}
& \quad \frac{1}{2} \leq \frac{1}{1-p(z)|\Omega| \hat{D}(k)}=\hat{C}_{p(z)}(k) \leq \hat{C}_{p(z)}(0)=\chi(z) \leq \chi(r), \\
& \left|\hat{G}_{z}(k)\right| \leq \chi(r),\left|\frac{\mathrm{d} \hat{G}_{z}(k)}{\mathrm{d} z}\right| \leq \chi^{\prime}(r),\left|\frac{\mathrm{d} \hat{C}_{p}(k)}{\mathrm{d} p}\right| \leq|\Omega| \chi(r)^{2} \text {, and } \frac{\mathrm{d} p(z)}{\mathrm{d} z} \leq|\Omega|^{-1} \chi^{\prime}(r) . \\
& \text { For } f_{3} \text {, it again suffices to show continuity in }[0, r] \text { for every } r<z_{c} \text {. Again } \\
& \text { we show equicontinuity on }[0, r] \text { for every } r<z_{c} \text {, by obtaining a uniform } \\
& \text { bound on the derivative with respect to } z \text {, and this follows as before. }
\end{aligned}
$$

Exercise 5.15. Fill in the missing details in the continuity proof of $f_{3}$.

Finally, we verify that $f$ obeys the substantial hypothesis of Lemma 5.9. 
Lemma 5.16. Fix $z \in\left(0, z_{c}\right)$ and suppose that $f(z) \leq 4$. If (5.2) holds with $\beta$ sufficiently small (independent of $z$ ), then it is in fact the case that $f(z) \leq$ $1+c \beta$ for some $c>0$ independent of $z$.

Proof. For $f_{1}(z)$, we simply note that $\chi(z)>0$ and hence, by (3.30),

$$
\chi(z)^{-1}=1-z|\Omega|-\hat{\Pi}_{z}(0)>0 .
$$

Therefore, by Lemma 5.11,

$$
f_{1}(z)=z|\Omega|<1-\hat{\Pi}_{z}(0) \leq 1+\bar{c}_{4} \beta
$$

if $\beta$ is sufficiently small.

For $f_{2}$, we first write $\hat{F}_{z}(k)=1 / \hat{G}_{z}(k)$, so that

$$
\frac{\hat{G}_{z}(k)}{\hat{C}_{p(z)}(k)}=\frac{1-p(z)|\Omega| \hat{D}(k)}{\hat{F}_{z}(k)}=1+\frac{1-p(z)|\Omega| \hat{D}(k)-\hat{F}_{z}(k)}{\hat{F}_{z}(k)} .
$$

We will show that the last term on the right hand side is $O(\beta)$, which implies that $f_{2}(z)=1+O(\beta)$.

We first obtain bounds on the numerator of the last term in (5.53), and afterwards consider the denominator. By (5.30) and (3.30), $p(z)|\Omega|=1-$ $\hat{F}_{z}(0)=z|\Omega|+\hat{\Pi}_{z}(0)$, and thus the numerator of the last term in (5.53) is

$$
1-p(z)|\Omega| \hat{D}(k)-\hat{F}_{z}(k)=\hat{\Pi}_{z}(0)[1-\hat{D}(k)]-\left[\hat{\Pi}_{z}(0)-\hat{\Pi}_{z}(k)\right] .
$$

This is bounded above by $4 \bar{c}_{4} \beta$, by (5.44). Additionally, by (5.44)-(5.45), it is also bounded above by

$$
\bar{c}_{4} \beta[1-\hat{D}(k)]+\bar{c}_{4} \beta[1-p(z)|\Omega| \hat{D}(k)] .
$$

Since

$$
[1-\hat{D}(k)] \hat{C}_{p(z)}(k)=1+\hat{D}(k) \frac{p(z)|\Omega|-1}{1-p(z)|\Omega| \hat{D}(k)} \leq 2,
$$

the numerator of (5.53) is bounded by

$$
3 \bar{c}_{4} \beta[1-p(z)|\Omega| \hat{D}(k)] \leq 3 \bar{c}_{4} \beta\left[\hat{F}_{z}(0)+[1-\hat{D}(k)]\right] .
$$

The denominator of (5.53) is

$$
\begin{aligned}
\hat{F}_{z}(k) & =\hat{F}_{z}(0)+\left[\hat{F}_{z}(k)-\hat{F}_{z}(0)\right] \\
& =\hat{F}_{z}(0)+z|\Omega|[1-\hat{D}(k)]+\left[\hat{\Pi}_{z}(0)-\hat{\Pi}_{z}(k)\right] .
\end{aligned}
$$

For $z \leq 1 / 2|\Omega|$, we use $\hat{F}_{z}(0) \geq \hat{C}_{z}(0)^{-1} \geq \frac{1}{2}, 1-\hat{D}(k) \geq 0$, and (5.44) to see that

$$
\hat{F}_{z}(k) \geq \hat{F}_{z}(0)-2 \bar{c}_{4} \beta \geq \frac{1}{2}-2 \bar{c}_{4} \beta
$$


For $1 / 2|\Omega| \leq z<z_{c}$, we use $\hat{F}_{z}(0)>0$, (5.45) and $1-p(z)|\Omega| \hat{D}(k)=$ $1-\left(1-\hat{F}_{z}(0)\right) \hat{D}(k) \leq 1-\hat{D}(k)+\hat{F}_{z}(0)$ to obtain

$$
\begin{aligned}
\hat{F}_{z}(k) & \geq \hat{F}_{z}(0)+\frac{1}{2}[1-\hat{D}(k)]-\bar{c}_{4} \beta[1-p(z)|\Omega| \hat{D}(k)] \\
& \geq\left[\frac{1}{2}-\bar{c}_{4} \beta\right]\left[\hat{F}_{z}(0)+[1-\hat{D}(k)]\right] .
\end{aligned}
$$

In either case, combining these inequalities with the bounds obtained above for the numerator of (5.53) gives $f_{2}(z)=1+O(\beta)$.

Finally, we consider $f_{3}$. We write

$$
\hat{g}_{z}(k)=z|\Omega| \hat{D}(k)+\hat{\Pi}_{z}(k),
$$

so that

$$
\hat{G}_{z}(k)=\frac{1}{1-\hat{g}_{z}(k)} .
$$

Note that $g_{z}(x)=g_{z}(-x)$, so we can apply Lemma 5.7 to obtain

$$
\begin{aligned}
\frac{1}{2}\left|\Delta_{k} \hat{G}_{z}(l)\right| \leq & \frac{1}{2}\left[\hat{G}_{z}(l-k)+\hat{G}_{z}(l+k)\right] \hat{G}_{z}(l)\left[\hat{g}_{z}^{\mathrm{av}}(0)-\hat{g}_{z}^{\mathrm{av}}(k)\right] \\
& +4 \hat{G}_{z}(l-k) \hat{G}_{z}(l) \hat{G}_{z}(l+k)\left[\hat{g}_{z}^{\mathrm{av}}(0)-\hat{g}_{z}^{\mathrm{av}}(k)\right]\left[\hat{g}_{z}^{\mathrm{av}}(0)-\hat{g}_{z}^{\mathrm{av}}(l)\right] .
\end{aligned}
$$

Using $f_{2}(z) \leq 1+O(\beta)$, we can bound each factor of $\hat{G}_{z}$ above by $[1+$ $O(\beta)] \hat{C}_{p(z)}$. Also,

$$
\begin{aligned}
\hat{g}_{z}^{\mathrm{av}}(0)-\hat{g}_{z}^{\mathrm{av}}(k) & \leq \sum_{x}[1-\cos (k \cdot x)]\left[z|\Omega| D(x)+\left|\Pi_{z}(x)\right|\right] \\
& \leq z|\Omega|[1-\hat{D}(k)]+\bar{c}_{4} \beta \hat{C}_{p(z)}(k)^{-1} \\
& \leq[2+O(\beta)] \hat{C}_{p(z)}(k)^{-1}
\end{aligned}
$$

using (5.45) for the second inequality, and $f_{1}(z) \leq 1+O(\beta)$ and (5.56) for the third. Combining these bounds gives $f_{3}(z) \leq 1+O(\beta)$.

This completes the proof that $f(z) \leq 1+O(\beta)$.

This completes the proof that $f$ obeys the hypotheses of Lemma 5.9, and also completes the proof of Theorem 5.8.

Exercise 5.17. (a) Give a monotonicity argument to conclude that the factor $\hat{C}_{p(z)}(k)^{-1}$ in $(5.45)$ can be replaced by $1-\hat{D}(k)$.

(b) Use the result of (a) to prove that $\sum_{x}|x|^{2}\left|\Pi_{z}(x)\right|$ is bounded above by $O\left(\beta \sigma^{2}\right)$ uniformly in $z<z_{c}$, where $\sigma^{2}=\sum_{x}|x|^{2} D(x)$.

(c) The correlation length of order $2, \xi_{2}(z)$, is defined by

$$
\xi_{2}(z)^{2}=\frac{1}{\chi(z)} \sum_{x}|x|^{2} G_{z}(x) .
$$

Prove that $\xi_{2}(z) \simeq\left(1-z / z_{c}\right)^{-1 / 2}$. 
As a final observation, we note that by (5.46) and dominated convergence, $\hat{\Pi}_{z_{c}}(k)$ is finite and is equal to the limit of $\hat{\Pi}_{z}(k)$ as $z$ approaches $z_{c}$ from the left. Since $\chi(z)$ diverges to infinity in this limit, it follows from (3.30) that

$$
1-z_{c}|\Omega|-\hat{\Pi}_{z_{c}}(0)=0
$$

and hence

$$
z_{c}=\frac{1}{|\Omega|}\left(1-\hat{\Pi}_{z_{c}}(0)\right)=\frac{1}{|\Omega|}+O\left(\frac{1}{|\Omega|^{2}}\right),
$$

where we have used $\left|\hat{\Pi}_{z_{c}}(0)\right| \leq O(\beta)=O\left(|\Omega|^{-1}\right.$ ) (and we assume $d>4$ for the spread-out model). For the spread-out model in dimensions $d>4$, an extension of (5.67) can be found in [118]. ${ }^{4}$ See [176] for related results for the spread-out model in dimensions $d \leq 4$. For the nearest-neighbour model, (5.67) is the first step in the proof of the asymptotic formula (2.8) for $\mu=1 / z_{c}$. The following exercise pushes (5.67) a bit further.

Exercise 5.18. Consider the nearest-neighbour model.

(a) Fix an integer $m \geq 1$. Show that $\left\|[1-\hat{D}]^{-m}\right\|_{1}$ is nonincreasing in $d>2 m$. Hint: $A^{-m}=\Gamma(m)^{-1} \int_{0}^{\infty} u^{m-1} e^{-u A} \mathrm{~d} u$.

(b) Let $H_{z}^{(j)}(x)=\sum_{m=j}^{\infty} c_{m}(x) z^{m}$. Show that $\left\|H_{z_{c}}^{(j)}\right\|_{\infty} \leq O\left(d^{-j / 2}\right)$, where the constant may depend on $j$. To do so, it is helpful first to show that $\left\|\hat{D}^{2 j}\right\|_{1} \leq c_{j}(2 d)^{-j}$ for some constant $c_{j}$ depending on $j=1,2, \ldots$.

(c) Prove that

$$
\hat{\Pi}_{z_{c}}^{(1)}(0)=\frac{1}{2 d}+\frac{3}{(2 d)^{2}}+O\left(\frac{1}{(2 d)^{3}}\right) .
$$

(d) Prove that

$$
\hat{\Pi}_{z_{c}}^{(2)}(0)=\frac{1}{(2 d)^{2}}+O\left(\frac{1}{(2 d)^{3}}\right) .
$$

(e) Conclude that the connective constant $\mu=z_{c}^{-1}$ obeys

$$
\mu=2 d-1-\frac{1}{2 d}+O\left(\frac{1}{(2 d)^{2}}\right)
$$

The strategy in this exercise is based on that used in [53, 122, 123], and is simpler than that used in [101]. Equation (5.70) was first proved in [140], using completely different methods.

\subsection{Finite Bubble vs Small Bubble}

According to Theorem 2.3, the susceptibility obeys the mean-field behaviour $\chi(z) \simeq\left(1-z / z_{c}\right)^{-1}$ if the critical bubble diagram $B\left(z_{c}\right)$ is finite. On the other hand, convergence of the lace expansion has been proved only when $B\left(z_{c}\right)-1$ is small. This leads to the restrictions that the dimension be large

\footnotetext{
${ }^{4}$ The results of [118] are expressed in terms of $p_{c}$ defined by $p_{c}=z_{c}|\Omega|$.
} 
for the nearest-neighbour model, or that $L$ be large for the spread-out model in dimensions $d>4$, in our use of Proposition 5.3 to drive the convergence proof.

For the nearest-neighbour model in dimension $d=5$, it was shown in $[97,98]$ that $B\left(z_{c}\right)-1 \leq 0.493$. This is small, although not very small. With considerable effort, and with a computer-assisted proof, convergence of the lace expansion was proved in $[97,98]$ for the nearest-neighbour model in dimensions $d \geq 5$.

It would be of great interest to find a proof of the bubble condition that would be applicable in situations where the bubble diagram could be large, rather than relying on it being small.

\subsection{Differential Equality and the Bubble Condition}

It is instructive now to revisit Theorem 2.3, which used inclusion-exclusion to give upper and lower bounds on the derivative of $z \chi(z)$. As in the proof of Lemma 5.16, we write

$$
\hat{F}_{z}(0)=\frac{1}{\chi(z)}=1-z|\Omega|-\hat{\Pi}_{z}(0) .
$$

Then direct calculation gives

$$
\frac{\mathrm{d}[z \chi(z)]}{\mathrm{d} z}=\left(\hat{F}_{z}(0)-z \frac{\mathrm{d} \hat{F}_{z}(0)}{\mathrm{d} z}\right) \frac{1}{\hat{F}_{z}(0)^{2}}=V(z) \chi(z)^{2},
$$

with

$$
V(z)=1+z \frac{\mathrm{d} \hat{\Pi}_{z}(0)}{\mathrm{d} z}-\hat{\Pi}_{z}(0) .
$$

The identity (5.72) gives an identity in place of the inequalities of (2.35), and corresponds to inclusion-exclusion carried out to all orders.

It is significant that $V\left(z_{c}\right)$ is finite, under the basic assumption of Section 5 that (5.2) is sufficiently small. We have already seen in Section 5.2 that $\hat{\Pi}_{z_{c}}(0)$ is finite. To see that $V\left(z_{c}\right)$ is finite, we must verify that the derivative $\mathrm{d} \hat{\Pi}_{z_{c}}(0) / \mathrm{d} z$ is also finite. Here is a sketch of a proof of this last fact.

It suffices to obtain a bound on

$$
\sum_{m=1}^{\infty} m \hat{\pi}_{m}^{(N)}(0) z_{c}^{m-1}
$$

which is summable in $N$. As in the proof of $(4.10)$, we associate to $\hat{\pi}_{m}^{(N)}(0)$ a diagram consisting of $2 N-1$ subwalks, whose lengths $m_{1}, \ldots, m_{2 N-1}$ sum to $m$. We decompose the factor $m$ in (5.74) as $m=\sum_{j=1}^{2 N-1} m_{j}$ and obtain a sum of $2 N-1$ terms. In the $j^{\text {th }}$ term, there is a factor $m_{j}$ associated to the $j^{\text {th }}$ 
line. We apply Lemma 4.6 to estimate the $j^{\text {th }}$ term, associating the infinity norm to the special line. Then we use the bound

$$
\begin{aligned}
\left\|\sum_{m=1}^{\infty} m c_{m}(x) z_{c}^{m-1}\right\|_{\infty} & =\left\|\mathrm{d} H_{z_{c}}(x) / \mathrm{d} z\right\|_{\infty} \\
& \leq\left\|H_{z_{c}} * G_{z_{c}}\right\|_{\infty} \leq\left\|H_{z_{c}}\right\|_{\infty}+\left\|H_{z_{c}}\right\|_{2}^{2},
\end{aligned}
$$

and draw the desired conclusion. The first inequality of (5.75) follows as in the upper bound of (2.35), and the second inequality is (4.11).

This shows that, as $z \rightarrow z_{c}^{-}$,

$$
\frac{\mathrm{d}[z \chi(z)]}{\mathrm{d} z} \sim V\left(z_{c}\right) \chi(z)^{2} .
$$

The left hand side is equal to the generating function of two mutually-avoiding self-avoiding walks starting from the origin. The asymptotic formula (5.76) shows that this generating function behaves in the same way as the generating function for two independent self-avoiding walks, up to a vertex factor $V\left(z_{c}\right)$ which takes into account the local effect of the mutual avoidance.

Exercise 5.19. Prove that when $\beta$ of (5.2) is sufficiently small, the susceptibility obeys the asymptotic formula

$$
\chi(z) \sim A\left(1-z / z_{c}\right)^{-1} \text { as } z \rightarrow z_{c}^{-},
$$

with $A=z_{c}^{-1}\left[|\Omega|+\frac{\mathrm{d}}{\mathrm{d} z} \hat{\Pi}_{z_{c}}(0)\right]^{-1}$. This improves the conclusion of Theorem 5.1 to an asymptotic formula, and also avoids any appeal to Theorem 2.3.

\section{Further Results for the Self-Avoiding Walk}

The proof of convergence of the lace expansion was presented in Section 5 in the context of proving the bubble condition, which is the simplest meaningful result that can be derived from convergence. However, it is possible to go substantially further, and in Section 6.1 we discuss several extensions for the self-avoiding walk in dimensions $d>4$. In Section 6.2, we discuss a result of van der Hofstad [108] for a 1-dimensional self-avoiding walk. In Section 6.3, we discuss a result of Ueltschi [204] for a self-avoiding walk with nearestneighbour attraction. Finally, in Section 6.4, we discuss applications of the lace expansion to networks of mutually-avoiding self-avoiding walks in dimensions $d>4$.

\subsection{The Self-Avoiding Walk in Dimensions $d>4$}

The lace expansion was invented by Brydges and Spencer [45] to prove the following theorem for the weakly self-avoiding walk (see (2.2) and (2.14)). 
Theorem 6.1. Let $d>4$ and consider the nearest-neighbour weakly selfavoiding walk. There is a $\lambda_{0}>0$ and an $\epsilon>0$ such that for all $0<\lambda \leq \lambda_{0}$ there are constants $A_{\lambda}$ and $v_{\lambda}$ such that for every $k \in \mathbb{R}^{d}$,

$$
\begin{aligned}
\hat{c}_{n}^{(\lambda)}\left(k / \sqrt{v_{\lambda} n}\right) & =A_{\lambda} \mu_{\lambda}^{n} \mathrm{e}^{-k^{2} / 2 d}\left[1+O\left(n^{-\epsilon}\right)\right], \\
\mathbb{E}_{n}^{(\lambda)}|\omega(n)|^{2} & =v_{\lambda} n\left[1+O\left(n^{-\epsilon}\right)\right] .
\end{aligned}
$$

The above result was revisited in [81, 144], where the lace expansion was treated as an example of a cluster expansion in statistical mechanics. The fact that this is a natural setting for the lace expansion has been emphasized in $[40,209]$.

A version of Theorem 6.1 was also obtained in [113], using a different approach to the lace expansion based on induction. The inductive approach notes that in the recursion

$$
\hat{c}_{n}(k)=|\Omega| \hat{D}(k) \hat{c}_{n-1}(k)+\sum_{m=1}^{n} \hat{\pi}_{m}(k) \hat{c}_{n-m}(k)
$$

of (3.26), the right hand side can be analyzed using only information about $\hat{c}_{j}(k)$ for $j<n$. In fact, this is clear for those $\hat{c}_{j}(k)$ occurring explicitly on the right hand side, and diagrammatic estimates can be used to bound $\hat{\pi}_{m}(k)$ using only $\hat{c}_{j}(k)$ for $j<m$. The results of [113] gives error estimates for $\hat{c}_{n}^{(\lambda)}(0)$ and the mean-square displacement that are likely optimal, namely

$$
\begin{aligned}
\hat{c}_{n}^{(\lambda)}(0) & =A_{\lambda} \mu_{\lambda}^{n}\left[1+O\left(n^{-(d-4) / 2}\right)\right], \\
\mathbb{E}_{n}^{(\lambda)}|\omega(n)|^{2} & = \begin{cases}v_{\lambda} n\left[1+O\left(n^{-1 \wedge(d-4) / 2}\right)\right] & (d \neq 5) \\
v_{\lambda} n\left[1+O\left(n^{-1} \log n\right)\right] & (d=5) .\end{cases}
\end{aligned}
$$

In particular, the power in the error term for $\hat{c}_{n}^{(\lambda)}(0)$ goes to infinity with the dimension.

In [120], an inductive analysis was used to show that under appropriate hypotheses, more general recursion relations have solutions with Gaussian asymptotics. The result of the inductive analysis is quite general, and has been applied in several contexts $[119,121,124,125,204]$. In particular, it was used in [124] to prove a version of Theorem 6.1 for the spread-out model (with $L$ of (1.2) sufficiently large) of strictly self-avoiding walks $(\lambda=1)$ in dimensions $d>4$. Such a result had been obtained previously in [158], using generating function methods rather than induction.

A variant of Theorem 6.1 was proved in [207] for a weakly self-avoiding version of a walk that takes steps of length $r$ parallel to the coordinate axes with probability proportional to $r^{-2}$. In this case, it was shown that for $d>2$ and for sufficiently small $\lambda$ there is convergence to the Cauchy distribution rather than to a Gaussian. A related result was obtained for a spread-out model in [52]. The fact that the upper critical dimension depends on the range of interaction was discussed in [10] for spin systems. 
In the series of papers $[183,184,185]$, it was shown that the conclusions of Theorem 6.1 hold for the nearest-neighbour strictly self-avoiding walk $(\lambda=1)$ if $d \geq d_{0}$, provided $d_{0}$ is sufficiently large. It was also proved that the scaling limit is Brownian motion in this case. This was improved in $[97,98]$ to give the following theorem (see [97] for additional statements). The proof of the theorem was computer assisted, and used precise numerical estimation of error terms to show that $d=5$ is large enough (barely!) to give convergence.

Theorem 6.2. Let $d \geq 5$ and $\lambda=1$ (strictly self-avoiding walk) and consider the nearest-neighbour model. There are constants $A$ and $v$ (depending on $d$ ) such that

$$
\begin{aligned}
c_{n} & =A \mu^{n}\left[1+O\left(n^{-\epsilon}\right)\right] \quad & \text { for any } \epsilon<\frac{d-4}{2} \wedge 1, \\
\mathbb{E}_{n}|\omega(n)|^{2} & =v n\left[1+O\left(n^{-\epsilon}\right)\right] \quad & \text { for any } \epsilon<\frac{d-4}{4} \wedge 1 .
\end{aligned}
$$

The scaling limit is Brownian motion, in the sense that if we linearly interpolate $(v n)^{-1 / 2} \omega$ to obtain a continuous function from $[0,1]$ to $\mathbb{R}^{d}$, then this converges weakly to Brownian motion. For $d=5,1 \leq A \leq 1.493$ and $1.098 \leq v \leq 1.803$

In [173], the diffusion constant $v$ is estimated to take the value $1.4767(13)$ for $d=5$.

The proofs of the above theorems are based on analysis of Fourier transforms, and they have the character of the central limit theorem. However, they do not provide a local central limit theorem, which is a statement that

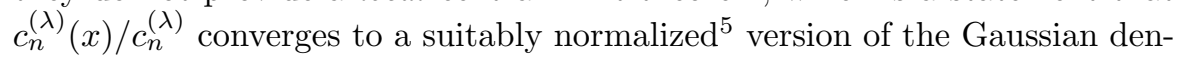
sity

$$
p_{n}(x)=\frac{d^{d / 2}}{(2 \pi n)^{d / 2}} \mathrm{e}^{-d|x|^{2} / 2 n},
$$

directly in $x$-space. A result in this direction was obtained by Bolthausen and Ritzmann [29], as stated in the following theorem. The method of [29] introduced a new approach to the analysis of the lace expansion, based on fixed-point methods.

Theorem 6.3. Let $d>4$ and consider the nearest-neighbour model. There are $\lambda_{0}>0, K>0$ and $\epsilon>0$ such that for all $0<\lambda \leq \lambda_{0}$ there is a $v$ (depending on $\lambda, d$ ) such that for all $n$ and $\|x\|_{1}$ of the same parity,

$$
\left|\frac{c_{n}^{(\lambda)}(x)}{c_{n}^{(\lambda)}}-2 p_{v n}(x)\right| \leq K\left[\frac{1}{\sqrt{n}} p_{v n}(x)+\frac{1}{n^{d / 2}} \sum_{j=1}^{n / 2} j p_{v j}(x)\right] .
$$

The factor 2 on the left hand side of (6.9) is there for the same parity reason that it is present for simple random walk (see, e.g., [149]). Namely, if

\footnotetext{
${ }^{5}$ The function $p_{n}(x)$ of $(6.8)$ is such that $\int_{\mathbb{R}^{d}}|x|^{2} p_{n}(x) \mathrm{d}^{d} x=n$ in all dimensions.
} 
we sum the first term on the left hand side over $x$ having the same parity as $n$, we get 1 , but we only get $\frac{1}{2}$ (approximately) if we sum $p_{v n}(x)$ over $x$ with fixed parity.

Exercise 6.4. The right hand side of (6.9) is somewhat opaque, and this exercise considers two consequences of (6.9).

(a) If we sum (6.9) over $n$, the Gaussian leading term $\sum_{n=0}^{\infty} 2 p_{v n}(x)$ behaves like a multiple of $|x|^{2-d}$. Summation of the error term gives as upper bound a multiple of

$$
\sum_{j=1}^{\infty}\left(\frac{1}{j^{(d+1) / 2}}+\frac{1}{j^{d-2}}\right) \mathrm{e}^{-d|x|^{2} / 2 v j} .
$$

By approximating the sum by an integral, this can be seen to be $O\left(|x|^{1-d}+\right.$ $\left.|x|^{6-2 d}\right)$. This is smaller than the leading term $|x|^{2-d}$, and thus provides a proof that $\eta=0$ (see (2.27)) under the assumptions of Theorem 6.3. Fill in the details of this discussion.

(b) Show that (6.9) implies that $c_{n}^{(\lambda)}(x) / c_{n}^{(\lambda)}$ is bounded above by a multiple of $n^{-d / 2}$.

In $[120,124]$, the following averaged version of a local central limit theorem is proved. For the averaging, we denote the cube of radius $R$ centred at $x \in \mathbb{Z}^{d}$ by

$$
C_{R}(x)=\left\{y \in \mathbb{Z}^{d}:\|x-y\|_{\infty} \leq R\right\} .
$$

We use $\lfloor x\rfloor$ to denote the closest lattice point in $\mathbb{Z}^{d}$ to $x \in \mathbb{R}^{d}$ (with an arbitrary rule to break ties).

Theorem 6.5. Consider the spread-out model of strictly self-avoiding walks. Let $d>4$. There is an $L_{0}=L_{0}(d)$ such that for $L \geq L_{0}$, the following holds. Let $R_{n}$ be any sequence with $\lim _{n \rightarrow \infty} R_{n}=\infty$ and $\lim _{n \rightarrow \infty} R_{n} n^{-1 / 2}=0$. Then for all $x \in \mathbb{R}^{d}$ with $x^{2}\left[\log R_{n}\right]^{-1}$ sufficiently small, as $n \rightarrow \infty$,

$$
\frac{1}{\left(2 R_{n}+1\right)^{d}} \sum_{y \in C_{R_{n}}(\lfloor x \sqrt{v n}\rfloor)} c_{n}(y)=A \mu^{n}\left(\frac{d}{2 \pi n v}\right)^{d / 2} \mathrm{e}^{-d x^{2} / 2}[1+o(1)] .
$$

Note that (6.12) is not an immediate consequence of the convergence of the Fourier transform of $c_{n}(x)$ to a Gaussian limit, which implies instead that sums over sets of volume $n^{d / 2}$ converge to integrals of the Gaussian density over the scaled set. The arbitrarily slow growth of $R_{n}$ in Theorem 6.5 probes $c_{n}(x)$ on a smaller scale.

See also Theorem 6.9 below for a related result, also proved using the inductive method of [120], which gives upper and lower bounds on $\sup _{x} c_{n}(x)$.

Alternate proofs of convergence of the lace expansion, which avoid use of Fourier transforms, were developed in [90, 91], where the following theorem was proved. Theorem 6.6 provides a statement that $\eta=0$ for the self-avoiding walk in dimensions $d>4$. 
Theorem 6.6. (a) [90] For $d \geq 5$, there is a constant $a^{\prime}$ depending on $d$, such that the critical two-point function of the nearest-neighbour strictly selfavoiding walk obeys, as $|x| \rightarrow \infty$,

$$
G_{z_{c}}(x)=\frac{a^{\prime}}{|x|^{d-2}}\left[1+O\left(\frac{1}{|x|^{2 / d}}\right)\right] .
$$

(b) [91] Fix any $\alpha>0$ (think of $\alpha$ small). For $d>4$, there is a finite constant a depending on $d$ and $L$, and an $L_{0}$ depending ond and $\alpha$, such that for $L \geq L_{0}$ the critical two-point function of the spread-out model of strictly self-avoiding walk obeys, as $|x| \rightarrow \infty$,

$$
G_{z_{c}}(x)=\frac{a}{|x|^{d-2}}\left[1+O\left(\frac{1}{|x|^{[2 \wedge 2(d-4)]-\alpha}}\right)\right] .
$$

The constant in the error term is uniform in $x$ but may depend on $L$ and $\alpha$; a more careful statement in this regard is given in [91].

The asymptotic formula $\xi(z) \sim \sqrt{v / 2 d}\left(1-z / z_{c}\right)^{-1 / 2}$ for the correlation length $(2.25)$ is proved in $[97,98]$ for the nearest-neighbour model in dimensions $d \geq 5$, using the method of [89]. The same result is obtained in [158] for the spread-out model in dimensions $d>4$ with $L$ sufficiently large. This confirms the prediction (2.26).

Finally, we mention a result concerning the infinite self-avoiding walk, a concept first defined in [147]. Given $n \geq m$ and an $m$-step self-avoiding walk $\omega$, let $P_{m, n}(\omega)$ denote the fraction of $n$-step walks which extend $\omega$, meaning that the first $m$ steps agree with $\omega$. Then we define

$$
P_{m}(\omega)=\lim _{n \rightarrow \infty} P_{m, n}(\omega)
$$

if the limit exists. If the limit does exist, then the probability measures $P_{m}$ on $m$-step walks will be consistent in the sense of Exercise 2.1. This consistency property allows for the definition via cylinder sets of a measure $P_{\infty}$ on the set of all infinite self-avoiding walks. The measure $P_{\infty}$ is the infinite self-avoiding walk. In [148], the lace expansion was used to construct the nearest-neighbour infinite self-avoiding walk in dimensions $d \geq d_{0}$, for some sufficiently large $d_{0}$. This was improved in [97] (see also [158]), where the nearest-neighbour infinite self-avoiding walk was constructed in all dimensions $d \geq 5$.

\subsection{A Self-Avoiding Walk in Dimension $d=1$}

The above results all consider $d>4$. An application to 1-dimensional nearestneighbour weakly self-avoiding walk has been given by van der Hofstad [108], and we discuss this next.

For $\lambda=1$, the 1-dimensional self-avoiding walk is easy: there are just two self-avoiding walks that start at the origin, one travelling left and one 
travelling right. However, for $\lambda \in(0,1)$, the problem is much more difficult. It was proved in [84] that the behaviour remains ballistic for all $\lambda \in(0,1)$, i.e., the walk is asymptotically at position $\theta n$ after $n$ steps, where the speed $\theta$ depends on $\lambda$. It remains an open problem to prove that $\theta$ is an increasing function of $\lambda$, although this seems obvious intuitively. The substantial recent progress in the study of 1-dimensional problems of this sort is reviewed in [116].

When $\lambda$ is close to 1 , the weak avoidance is actually rather strong. In the following theorem, due to [108], the lace expansion was used to treat the weakly self-avoiding walk, with $\lambda$ close to 1 , as a small perturbation of the one-dimensional strictly self-avoiding walk. This is the only time that the lace expansion has been used to perturb around a non-Gaussian model.

We replace $\lambda$ by

$$
\lambda=\lambda_{s t}=1-\exp \left[-\beta|s-t|^{-p}\right],
$$

with $p \in[0,1]$. For factor $|s-t|^{-p}$ has the effect of diminishing $\beta$ when $p \in(0,1]$. This corresponds to a "forgetful" self-avoidance, in the sense that the penalty for a self intersection decreases when the time until the selfintersection increases. Let

$$
\hat{c}_{n}^{+}(k)=\sum_{x \geq 0} c_{n}(x) \mathrm{e}^{\mathrm{i} k x} .
$$

This breaks the left-right symmetry, which is necessary if we are to observe ballistic behaviour.

Theorem 6.7. Let $d=1$ and $p \in[0,1]$, and consider the nearest-neighbour model with $\lambda=\lambda_{\text {st }}$ given by (6.16). There is a (large) $\beta_{0}$ such that for all $\beta \geq \beta_{0}$ there exists $v=v(\beta, p)$ and $\theta=\theta(\beta, p)$ such that

$$
\lim _{n \rightarrow \infty} \mathrm{e}^{-\mathrm{i} \theta n k / v \sqrt{n}} \frac{\hat{c}_{n}^{+}(k / v \sqrt{n})}{\hat{c}_{n}^{+}(0)}=\mathrm{e}^{-k^{2} / 2} .
$$

The first factor on the left hand side of (6.18) is an indication of ballistic behaviour with speed $\theta$. Extensions of (6.18) are given in [108]. See [138] for earlier work on this model. It was shown in [113] that if $p>\frac{3}{2}$ and $\lambda$ is small then the ballistic behaviour of Theorem 6.7 is replaced by diffusive behaviour. Arguments were put forward in [47] that for $\lambda>0$ and $1 \leq p \leq \frac{3}{2}$ the mean-square displacement is asymptotic to a multiple of $n^{2 \nu_{p}}$, where $\nu_{p}$ varies linearly in $p$ between $\nu_{1}=1$ and $\nu_{3 / 2}=\frac{1}{2}$. Extensions to higher dimensions are also discussed in [47].

\subsection{Nearest-Neighbour Attraction}

The self-avoiding walk models a single polymer in a good solution. In a bad solution, it is energetically more favourable for the polymer to be in contact with itself rather than with the solution. This is modelled as follows. 
We fix $\kappa>0$ and replace $U_{s t}$ of $(2.1)$ by

$$
V_{s t}(\omega)=\left\{\begin{aligned}
-\lambda & \text { if } \omega(s)=\omega(t) \\
\kappa & \text { if }\|\omega(s)-\omega(t)\|_{1}=1 \\
0 & \text { otherwise. }
\end{aligned}\right.
$$

Define $D: \mathbb{Z}^{d} \rightarrow \mathbb{R}$ by $D(0)=0, D(x)=a_{L} \mathrm{e}^{-|x| / L}$ for $x \neq 0$, where $a_{L}$ is chosen so that $\sum_{x \in \mathbb{Z}^{d}} D(x)=1$. (In fact, the theorem below, due to [204], allows for a more general choice of $D$.) For an $n$-step walk $\omega$, let $W(\omega)=$ $\prod_{j=1}^{n} D(\omega(j)-\omega(j-1))$. Let

$$
c_{n}^{(\lambda, \kappa)}(x)=\sum_{\omega \in \mathcal{W}_{n}(0, x)} W(\omega) \prod_{0 \leq s<t \leq n}\left(1+V_{s t}(\omega)\right),
$$

where the sum is over $n$-step walks taking arbitrary steps. Let $c_{n}^{(\lambda, \kappa)}=$ $\sum_{x \in \mathbb{Z}^{d}} c_{n}^{(\lambda, \kappa)}(x)$. Then we define a measure by

$$
\mathbb{E}_{n}^{(\lambda, \kappa)} X=\frac{1}{c_{n}^{(\lambda, \kappa)}} \sum_{\omega \in \mathcal{W}_{n}} X(\omega) W(\omega) \prod_{0 \leq s<t \leq n}\left(1+V_{s t}(\omega)\right) .
$$

In (6.19), there is a competition between two tendencies. The self-avoidance tends to make the walk more spread out, whereas the nearest-neighbour attraction tends to make the walk more compact. The general picture $[132,205]$ is that as $\kappa$ is increased, with $\lambda$ fixed, there is a collapse transition at a critical value $\kappa_{c}=\kappa_{c}(\lambda)$. In this transition the walk's length scale (measured e.g. by average end-to-end distance) jumps from being of order $n^{\nu}$ with $\nu$ given by (2.16) when $\kappa<\kappa_{c}$, to order $n^{1 / d}$ for $\kappa>\kappa_{c}$. The critical value $\kappa_{c}$ is known as the theta point, and at the theta point it is predicted that the two competing effects cancel each other, so that (for $d \geq 3$ ) the length scale is the Gaussian length scale $n^{1 / 2}$. For simulations of the collapse transition in dimension $d=5$, see [174].

The following theorem of Ueltshi [204] proves that there is an uncollapsed phase in the above model, for $d>4, \lambda=1$ and small $\kappa$. This theorem is noteworthy in its application to a model for which the interaction is not purely repulsive. The attractive nature of the interaction is problematic, as the inequality $1+\mathcal{U}_{s t} \leq 1$ used in the proof of existence of the connective constant, and of Proposition 4.2, can now be violated. Specific properties of the choice of $D$ above are used in the proof of the theorem, to surmount this difficulty.

Theorem 6.8. Fix $6>4, \delta \in\left(0,1 \wedge \frac{d-4}{2}\right)$, and let $V$ be as above. There is an $L_{0}$ and a $\kappa_{0}$ such that for all $L \geq L_{0}$ and $0<\kappa \leq \kappa_{0}$ there are positive constants $A=A(\kappa, L, d), \mu=\mu(\kappa, L, d)$ and $v=v(\kappa, L, d)$ such that

\footnotetext{
${ }^{6}$ The restriction on $\delta$ in [120, Theorem 1.1] contains a misprint and should involve $\frac{d-4}{2}$ rather than $\frac{d-4}{4}$.
} 


$$
\begin{aligned}
\hat{c}_{n}^{(1, \kappa)}(0) & =A \mu^{n}\left[1+O\left(n^{-(d-4) / 2}\right)\right], \\
\mathbb{E}_{n}^{(1, \kappa)}|\omega(n)|^{2} & =v n\left[1+O\left(n^{-\delta}\right)\right] .
\end{aligned}
$$

The proof makes use of the general inductive theorem of [120], and, as such, has consequences that go beyond (6.22)-(6.23).

\subsection{Networks of Self-Avoiding Walks}

Polymer networks can be modelled by networks of mutually-avoiding selfavoiding walks. The scaling behaviour of such networks has been studied in the physics literature for general dimensions [66, 67], but mathematically rigorous results are restricted to $d>4$. Networks in dimensions $d>4$ having the topology of a tree were studied in [124]. A special case of the results of [124] pertains to star polymers, which we discuss now.

Let $r \geq 1, \vec{n}=\left(n_{1}, \ldots, n_{r}\right)$ with each $n_{j}$ a positive integer, and $\vec{x}=$ $\left(x_{1}, \ldots, x_{r}\right)$ with each $x_{j} \in \mathbb{Z}^{d}$. Let $s_{\vec{n}}^{(r)}(\vec{x})$ denote the number of star polymers consisting of $r$ self-avoiding walks of length $n_{1}, \ldots, n_{r}$ starting at the origin and ending at $x_{1}, \ldots, x_{r}$ respectively, which are also mutually avoiding apart from their common beginning at the origin. We consider the model in which the walks take steps in the spread-out set $\Omega$ of (1.2). See Fig. 6.1. Let $s_{\vec{n}}^{(r)}=$ $\sum_{\vec{x} \in \mathbb{Z}^{d r}} s_{\vec{n}}^{(r)}(\vec{x})$.
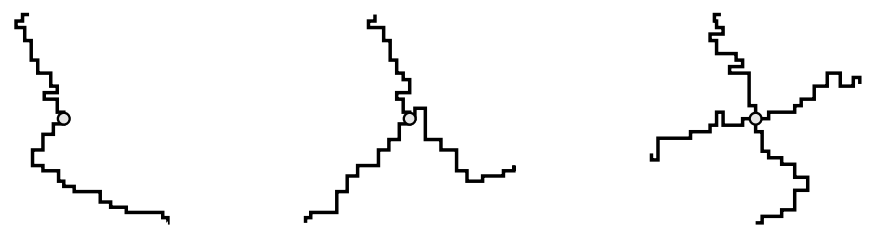

Fig. 6.1. Star polymers with $r=2,3,4$.

Theorem 6.9. Let $^{7} d>4, \delta \in\left(0,1 \wedge \frac{d-4}{2}\right), r \geq 1, \vec{n}=\left(n_{1}, \ldots, n_{r}\right)$, and $n=\sum_{j=1}^{r} n_{j}$, and assume that $n_{j} \sim n t_{j}$ with $t_{j} \in(0,1]$ for each $j$. There is an $L_{0}=L_{0}(d)$ such that for $L \geq L_{0}$ there exist positive constants $v, \mu, A$, $V_{r}$ (all depending on $d$ and $L$ ) with $V_{1}=1$ and $V_{2}=A^{-1}$, and there exist $C_{1}, C_{2}$ (depending on d but not $L$ ), such that the following statements hold as $n \rightarrow \infty$ :

$$
s_{\vec{n}}^{(r)}=V_{r} A^{r} \mu^{n}\left[1+O\left(n^{-(d-4) / 2}\right)\right],
$$

\footnotetext{
${ }^{7}$ The footnote to Theorem 6.8 applies also here.
} 


$$
\begin{aligned}
\frac{1}{s_{\vec{n}}^{(r)}} \sum_{\vec{x} \in \mathbb{Z}^{d r}}\left|x_{j}\right|^{2} s_{\vec{n}}^{(r)}(\vec{x}) & =v n_{j}\left[1+O\left(n^{-\delta}\right)\right] \quad(j=1, \ldots, r), \\
C_{1} \mu^{n} L^{-d r} n^{-d r / 2} & \leq \sup _{\vec{x} \in \mathbb{Z}^{d r}} s_{\vec{n}}^{(r)}(\vec{x}) \leq C_{2} \mu^{n} L^{-d r} n^{-d r / 2} .
\end{aligned}
$$

Since (6.24) with $r=1$ gives $s_{n}^{(1)}=c_{n}=A \mu^{n}\left[1+O\left(n^{-(d-4) / 2}\right)\right]$, $\mu$ must be the connective constant given by $\mu=\lim _{n \rightarrow \infty} c_{n}^{1 / n}$. Thus the connective constant $\mu$ also serves as the growth constant for star polymers. For $d=3$, a closely related result is proved in [194]; the proof extends to general $d \geq 2$ [193]. Also, (6.25) gives $c_{n}^{-1} \sum_{x \in \mathbb{Z}^{d}}|x|^{2} c_{n}(x)=v n\left[1+O\left(n^{-\delta}\right)\right]$, so $v$ is the diffusion constant.

Theorem 6.9 states that the constants $A$ and $V_{2}$ are related by $V_{2}=A^{-1}$. In fact, this is required for consistency of (6.24). To see this, consider the statement of (6.24) for $r=1$ and $n=2 m$. In this case, (6.24) gives $c_{2 m} \sim$ $A \mu^{2 m}$. On the other hand, we may regard a single polymer of length $2 m$ as a star polymer consisting of two branches of length $m$. With this interpretation, (6.24) gives $c_{2 m} \sim A^{2} V_{2} \mu^{2 m}$. Therefore it must be the case that $V_{2}=A^{-1}$.

The constants $V_{r}$ are referred to as vertex factors. It is proved in [124] that $V_{r}=1+O\left(L^{-d}\right)$ for $r \geq 2$. Although not explicitly stated in [124], it follows easily from the results of [124] that there is a positive constant $c_{r}$ such that

$$
V_{r} \leq 1-c_{r} L^{-d} \quad(r \geq 2) .
$$

The vertex factors take into account the local effect of the mutual avoidance of the walks that meet at the origin, and (6.27) reflects the natural fact that the mutual avoidance diminishes the number of allowed configurations. This is analogous to (5.76), and, in fact, it is the case that $V\left(z_{c}\right)$ of $(5.76)$ is equal to $V_{2}$.

Since $s_{n}^{(1)}(x)=c_{n}(x),(6.26)$ provides both upper and lower bounds for $c_{n}(x)$ of the form $n^{-d / 2}$. This is a kind of local central limit theorem (cf. Theorems 6.3 and 6.5).

In [126], general graphical networks were considered, possibly containing closed loops. The results of [126] are stated in terms of critical generating functions. For simplicity, we state the result of [126] as it applies to the example of watermelon networks. See Fig. 6.2. For $r \geq 2$, let

$$
W_{z}^{(r)}(x)=\sum_{\left(\omega_{1}, \ldots, \omega_{r}\right) \in \mathcal{S}^{(r)}(0, x)} z^{\left|\omega_{1}\right|+\cdots+\left|\omega_{r}\right|},
$$

where the sum is over all $r$-tuples of self-avoiding walks from 0 to $x$, of any length, which are mutually avoiding apart from their common endpoints 0 and $x$. The walks are spread-out walks with steps given by (1.2).

Theorem 6.10. Let $d>4$ and $r \geq 2$. Fix $\epsilon_{1}<(d-4) \wedge 1$. Let $z_{c}$ be the critical value, let $V_{r}$ be the vertex factors of Theorem 6.9, and let a be the 

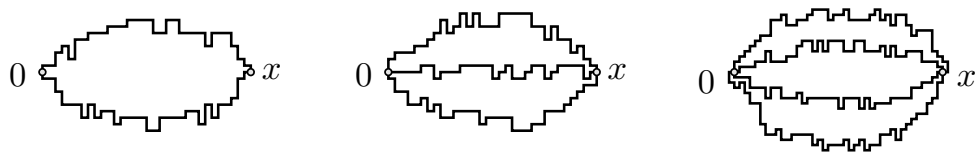

Fig. 6.2. Watermelon networks with $r=2,3,4$.

constant of (6.14). There exists an $L_{0}=L_{0}(d, r)$ such that for $L \geq L_{0}$, as $|x| \rightarrow \infty$

$$
W_{z_{c}}^{(r)}(x)=V_{r}^{2} \frac{a^{r}}{|x|^{r(d-2)}}\left[1+O\left(\frac{1}{|x|^{\epsilon_{1}}}\right)\right] .
$$

Constants in the error term depend on $L, d, r$ and $\epsilon_{1}$.

Comparing with the asymptotic formula for the critical two-point function in Theorem 6.6, we can interpret Theorem 6.10 as stating that the leading asymptotic behaviour of the watermelon network is that of $r$ independent self-avoiding walks joining 0 to $x$, apart from the vertex factors associated with the two vertices of the watermelon network. The results of [126] provide a similar conclusion for networks with the topology of an arbitrary graph.

The proofs of Theorems 6.9-6.10 are based on an extension of the notion of laces on an interval, as defined in Section 3.3, to laces on a tree. A general theory of such laces is developed in [124].

\section{Lattice Trees}

Lattice trees are natural combinatorial objects that fit within the general framework of critical phenomena. They also model branched polymers. In this section, we first give an overview of the predicted scaling properties of lattice trees and state results obtained in dimensions $d>8$ using the lace expansion. We then show that the square condition plays a similar role for lattice trees as the bubble condition does for the self-avoiding walk.

\subsection{Asymptotic Behaviour}

A lattice tree on $\mathbb{Z}^{d}$ is defined to be a finite connected set of bonds which contains no cycles (closed loops). Bonds are pairs $\{x, y\}$ of vertices of $\mathbb{Z}^{d}$, with $y-x \in \Omega$, where $\Omega$ is given either by the nearest-neighbour set (1.1) or the spread-out set (1.2). Although a tree $T$ is defined as a set of bonds, we will write $x \in T$ if $x$ is an element of a bond in $T$. The number of bonds in $T$ is denoted $|T|$, and the number of vertices in $T$ is thus $|T|+1$.

A basic combinatorial problem is to count the number of lattice trees of fixed size. Let $t_{n}^{(2)}(x)$ denote the number of $n$-bond lattice trees that contain 


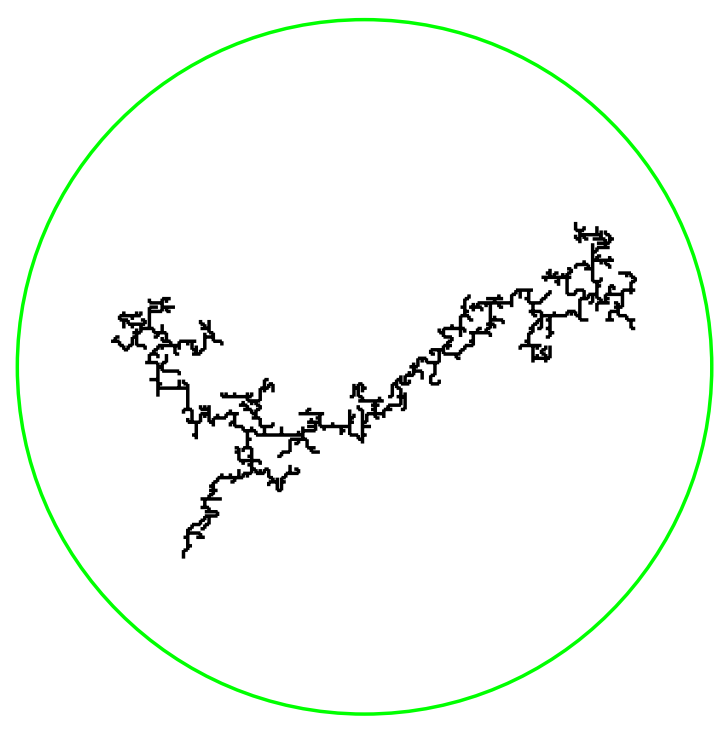

Fig. 7.1. A 2-dimensional lattice tree with $n=1000$ bonds, generated using the algorithm of [133]. The circle has radius $n^{0.64}$.

the two vertices 0 and $x$, and let $t_{n}^{(1)}=t_{n}^{(2)}(0)$ denote the number of $n$-bond trees that contain the origin. Note that

$$
\begin{aligned}
\hat{t}_{n}^{(2)}(0) & =\sum_{x \in \mathbb{Z}^{d}} t_{n}^{(2)}(x)=\sum_{x \in \mathbb{Z}^{d}} \sum_{T:|T|=n, T \ni 0, x} 1 \\
& =\sum_{T:|T|=n, T \ni 0} \sum_{x \in T} 1=(n+1) t_{n}^{(1)}(0) .
\end{aligned}
$$

It is customary to count lattice trees modulo translation, namely to consider $t_{n}$ defined by

$$
t_{n}=\frac{1}{n+1} t_{n}^{(1)}=\frac{1}{(n+1)^{2}} \hat{t}_{n}^{(2)}(0) .
$$

A subadditivity argument [145] shows that there is a positive constant $\lambda$ such that $\lim _{n \rightarrow \infty} t_{n}^{1 / n}=\lambda$. The precise asymptotic behaviour of $t_{n}$ as $n \rightarrow \infty$ is believed to be given by

$$
t_{n} \sim \operatorname{const} \lambda^{n} n^{-\theta}
$$

where $\theta$ is a universal critical exponent. The bounds

$$
c_{1} n^{-c_{2} \log n} \lambda^{n} \leq t_{n} \leq c_{3} n^{-(d-1) / d} \lambda^{n},
$$


proved respectively in [131] and [157], are the best general bounds known at present for $t_{n}$.

In studying $t_{n}$, it is convenient to introduce the two-point function

$$
G_{z}(x)=\sum_{n=0}^{\infty} t_{n}^{(2)}(x) z^{n}=\sum_{T: T \ni 0, x} z^{|T|},
$$

and the susceptibility $\chi(z)=\sum_{x} G_{z}(x)=\hat{G}_{z}(0)$. The susceptibility has radius of convergence $z_{c}=\lambda^{-1}$. It is a consequence of $(7.2)-(7.3)$ that $\hat{t}_{n}^{(2)}(0) \sim$ const $\lambda^{n} n^{2-\theta}$, and this corresponds, at least formally, to a singularity

$$
\chi(z) \sim \operatorname{const} \frac{1}{\left(1-z / z_{c}\right)^{\gamma}}
$$

for the susceptibility, with $\gamma=3-\theta$. As in (2.27)-(2.28), it is predicted that

$$
G_{z_{c}}(x) \sim \operatorname{const} \frac{1}{|x|^{\mid-2+\eta}}
$$

as $|x| \rightarrow \infty$, and that

$$
\hat{G}_{z_{c}}(k) \sim \operatorname{const} \frac{1}{|k|^{2-\eta}}
$$

as $k \rightarrow 0$, where $\eta$ is universal.

The typical length scale of a lattice tree is characterized by the average radius of gyration $R_{n}$, defined as follows. The squared average radius of gyration is defined by

$$
R_{n}^{2}=\frac{1}{t_{n}^{(1)}} \sum_{T:|T|=n, T \ni 0} R(T)^{2}
$$

where

$$
R(T)^{2}=\frac{1}{|T|+1} \sum_{x \in T}\left|x-\bar{x}_{T}\right|^{2}
$$

is the squared radius of gyration of $T$. In $(7.10), \bar{x}_{T}=(|T|+1)^{-1} \sum_{x \in T} x$ denotes the centre of mass of $T$ (considered as a set of equal masses at the vertices of $T$ ).

Exercise 7.1. Show that

$$
R_{n}^{2}=\frac{1}{2 \hat{t}_{n}^{(2)}(0)} \sum_{x}|x|^{2} t_{n}^{(2)}(x) .
$$

It is predicted that there is a universal critical exponent $\nu$ such that

$$
R_{n} \sim \operatorname{const} n^{\nu} .
$$

It is also believed that the critical exponents $\gamma, \eta, \nu$ are related by Fisher's relation $\gamma=(2-\eta) \nu$. In principle, to construct a scaling limit of lattice trees 
the lattice spacing should be rescaled from 1 to $n^{-\nu}$, followed by the limit $n \rightarrow \infty$. It is not immediately obvious how to describe the scaling limit of lattice trees, but a useful description for $d>8$ will be discussed in Section 16.4.

A lattice animal is a connected set of bonds which may contain closed loops. It is believed that lattice trees belong to the same universality class as lattice animals, so that lattice trees and lattice animals have the same critical exponents and scaling limits. A definition of weakly self-avoiding lattice trees was given in [30], which presumably is also in the same universality class.

A model of continuous branched polymers, which is also expected to be in the same universality class, was proposed by Brydges and Imbrie and analyzed in [41, 42]. Inspired by ideas of Parisi and Sourlas [175], Brydges and Imbrie [41] proved existence of critical exponents for their continuum model in dimensions $d=2$ and 3 (with partial results for $d=4$ ), with values $\theta=1$ for $d=2$, and $\theta=\frac{3}{2}, \nu=\frac{1}{2}, \eta=-1$ for $d=3$. It is believed that two-dimensional lattice trees do not have a conformally invariant scaling limit, and that they are not among the substantial class of models described by the SchrammLoewner evolution $\mathrm{SLE}_{\kappa}$. It has been conjectured [36] that $\eta$ is negative for all dimensions $2<d<8$.

Based on a field theoretic representation, it was argued in [155] that the upper critical dimension for lattice trees is 8 . Further evidence for this was given in $[36,199,201]$. The mean-field values of the exponents are $\gamma=\frac{1}{2}$, $\nu=\frac{1}{4}$ and $\eta=0$. The value $\nu=\frac{1}{4}$ corresponds in (7.12) to $n \sim \operatorname{const} R_{n}^{4}$, which is a statement of 4-dimensionality. The fact that two 4-dimensional objects generically do not intersect in more than eight dimensions gives a quick prediction that $d=8$ is the upper critical dimension for lattice trees.

The lace expansion has been used to prove a number of results for lattice trees in dimensions $d>8$. The following theorem [90, 91] shows that $\eta=$ 0 for nearest-neighbour lattice trees in sufficiently high dimensions, and for sufficiently spread-out models when $d>8$. Similar results are proved in $[90,91]$ for lattice animals.

Theorem 7.2. (a) [90] There is a $d_{0}$ such that for $d \geq d_{0}$, there is a constant $a^{\prime}$ depending on $d$, such that the critical lattice tree two-point function obeys, as $|x| \rightarrow \infty$,

$$
G_{z_{c}}(x)=\frac{a^{\prime}}{|x|^{d-2}}\left[1+O\left(\frac{1}{|x|^{2 / d}}\right)\right] .
$$

(b) [91] Let $d>8$, fix any $\alpha>0$ (think of $\alpha$ small), and consider the spreadout model of lattice trees. There is a finite constant a depending on $d$ and $L$, and an $L_{0}$ depending on $d$ and $\alpha$, such that for $L \geq L_{0}$, as $|x| \rightarrow \infty$,

$$
G_{z_{c}}(x)=\frac{a}{|x|^{d-2}}\left[1+O\left(\frac{1}{|x|^{[2 \wedge(d-8)]-\alpha}}\right)\right] .
$$

The constant in the error term is uniform in $x$ but may depend on $L$ and $\alpha$; a more careful statement in this regard is given in [91]. 
The following theorem, proved in [99], proves that $\theta=\frac{5}{2}$ and $\nu=\frac{1}{4}$ in high dimensions. Weaker results have been obtained for lattice animals, at the level of generating functions [95].

Theorem 7.3. For nearest-neighbour lattice trees with d sufficiently large, or for spread-out lattice trees with $d>8$ and $L$ sufficiently large, there are positive constants $A$ and $D$ (depending on $d, L$ ) such that for every $\epsilon<\frac{1}{2} \wedge \frac{d-8}{4}$,

$$
\begin{aligned}
t_{n} & =A \lambda^{n} n^{-5 / 2}\left[1+O\left(n^{-\epsilon}\right)\right], \\
R_{n} & =D n^{1 / 4}\left[1+O\left(n^{-\epsilon}\right)\right] .
\end{aligned}
$$

As is the proof of Theorem 6.2, the proof of Theorem 7.3 proceeds first by studying the singularity of $\hat{G}_{z}(k)$ at $z=z_{c}=\lambda^{-1}$, and then uses complex variable methods to extract the asymptotics of $t_{n}$ and the radius of gyration. In particular, it is shown that under the hypotheses of the theorem the susceptibility obeys

$$
\chi(z)=\frac{A \sqrt{\pi}}{\sqrt{1-z / z_{c}}}+O\left(\left|1-z / z_{c}\right|^{-1 / 2+\epsilon}\right)
$$

for all complex $|z| \leq z_{c}$, which implies that $\gamma$ exists and equals $\frac{1}{2}$.

The scaling limit of lattice trees in dimensions $d>8$ will be discussed in Section 16.4, but the following theorem of [62] gives a first step. For the statement, let $A$ and $D$ be the constants of Theorem 7.3, and let

$$
D_{0}=2^{3 / 4} \pi^{-1 / 4} D .
$$

Theorem 7.4. For nearest-neighbour lattice trees with $d$ sufficiently large, or for spread-out lattice trees with $d>8$ and $L$ sufficiently large, as $n \rightarrow \infty$,

$$
\hat{t}_{n}^{(2)}\left(k D_{0}^{-1} n^{-1 / 4}\right) \sim A \lambda^{n} n^{-1 / 2} \int_{0}^{\infty} \mathrm{d} t t \mathrm{e}^{-t^{2} / 2} \mathrm{e}^{-|k|^{2} t / 2 d} .
$$

The integral on the right hand side of (7.19) will be discussed in Section 16. Spatial scaling by $n^{-1 / 4}$ corresponds to replacement of the Fourier variable $k$ by $k n^{-1 / 4}$, and (7.19) is a statement about the scaling limit of $t_{n}^{(2)}(x)$ in Fourier language. Note that (7.15) can be combined with (7.2) to give $\hat{t}_{n}^{(2)}(0) \sim$ $A \lambda^{n} n^{-1 / 2}$, which is consistent with (7.19).

We will not prove Theorems 7.2-7.4 here. The proofs involve deriving a lace expansion for lattice trees, and diagrammatic estimates for the expansion, followed by proof of convergence of the expansion. The latter can be handled in various ways $[91,95,99,125]$. Significant new issues do arise in the proofs of Theorems 7.3-7.4. The modifications needed to apply the lace expansion to lattice trees will be discussed in Section 8. But first, we indicate how the role of the bubble condition for self-avoiding walks is replaced by the square condition for lattice trees. 


\subsection{Differential Inequalities and the Square Condition}

In this section, we adapt the method and results of Section 2.2 to lattice trees. For $r \geq 1$, we define the $r$-point function by

$$
G_{z}^{(r)}\left(x_{1}, \ldots, x_{r-1}\right)=\sum_{T: T \ni 0, x_{1}, \ldots, x_{r-1}} z^{|T|} .
$$

In particular, $G_{z}^{(2)}(x)$ is the two-point function $G_{z}(x)$ of (7.5), and we will write $g_{z}=\sum_{T: T \ni 0} z^{|T|}$ for the one-point function. For $r \geq 1$,

$$
\begin{aligned}
\frac{\mathrm{d}\left[z G_{z}^{(r)}\left(x_{1}, \ldots, x_{r-1}\right)\right]}{\mathrm{d} z} & =\sum_{T: T \ni 0, x_{1}, \ldots, x_{r-1}}(|T|+1) z^{|T|} \\
& =\sum_{x_{r}} G_{z}^{(r+1)}\left(x_{1}, \ldots, x_{r}\right) .
\end{aligned}
$$

For $r=1,2$, this implies that

$$
\frac{\mathrm{d}\left[z g_{z}\right]}{\mathrm{d} z}=\sum_{x \in \mathbb{Z}^{d}} G_{z}(x)=\chi(z),
$$

and

$$
\frac{\mathrm{d}[z \chi(z)]}{\mathrm{d} z}=\sum_{x, y} G_{z}^{(3)}(x, y) .
$$

We seek upper and lower bounds for the right hand side of (7.23), which will provide differential inequalities for the susceptibility.

For an upper bound, we note that a lattice tree containing vertices $0, x, y$ contains a unique skeleton consisting of the union of the paths in the tree joining 0 to $x$, and 0 to $y$. The skeleton divides naturally into three paths, which join each of $0, x, y$ to the vertex $u$ (say) where the path from 0 to $y$ separates from the path from 0 to $x$. By neglecting the mutual avoidance of the three subtrees defined naturally by these paths (the "inclusion" part of inclusion-exclusion), we obtain

$$
G_{z}^{(3)}(x, y) \leq \sum_{u} G_{z}(u) G_{z}(x-u) G_{z}(y-u) .
$$

Inserting this into (7.23) then gives the upper bound

$$
\frac{\mathrm{d}[z \chi(z)]}{\mathrm{d} z} \leq \chi(z)^{3} .
$$

For a lower bound, we first define the square diagram by

$$
\mathrm{S}(z)=\sum_{x, y, u} G_{z}(x) G_{z}(y-x) G_{z}(u-y) G_{z}(u) .
$$


Using the inverse Fourier transform, it follows from Exercise 1.1 that

$$
\mathrm{S}(z)=\int_{[-\pi, \pi]^{d}} \hat{G}_{z}(k)^{4} \frac{\mathrm{d}^{d} k}{(2 \pi)^{d}} .
$$

The square condition states that $\mathrm{S}\left(z_{c}\right)<\infty$, and will be satisfied for $d>8$ if $\eta=0$ in (7.7)-(7.8). Keeping only the term $x=y=u=0$ in (7.26), we see that $\mathrm{S}(z) \geq g_{z}^{4} \geq g_{z}$, and hence the square condition implies that $g_{z_{c}}<\infty$.

For the "exclusion" part of the inclusion-exclusion, we note two sources of overcounting in (7.24). One source is the fact that each two-point function on the right hand side of (7.24) contributes a branch at $u$, and this overcounting can be handled by inserting a factor $1 / g_{z}^{2}$ to prune two of the branches. Another source arises from the possible intersection of the three subtrees. Bounding this subtracted term from above, we are led to the lower bound depicted in Fig. 7.2 (see [95, Section 1.3] for more details). Summing this lower bound gives

$$
\chi(z)^{3}\left[\frac{1}{g_{z}^{2}}-3\left[\mathrm{~S}(z)-g_{z}^{4}\right]\right] \leq \frac{\mathrm{d}[z \chi(z)]}{\mathrm{d} z} \leq \chi(z)^{3} .
$$

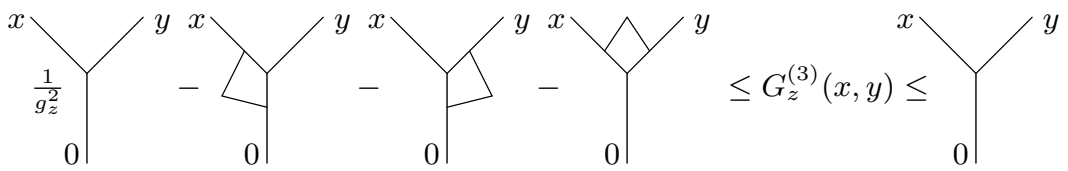

Fig. 7.2. The "skeleton inequalities" (7.28) for lattice trees.

The diagrams in Fig. 7.2 have a dual interpretation. On the one hand, they give a schematic depiction of actual tree configurations (or of tree-like configurations when self-intersections are imposed on the left hand side). On the other hand, the diagrams have the interpretation that each line represents a two-point function, with unlabelled vertices summed over $\mathbb{Z}^{d}$. The latter is an upper bound on generating functions of the actual configurations, and it is the latter interpretation that is literally intended in Fig. 7.2. It is nevertheless instructive to keep the former interpretation in mind also.

Exercise 7.5. Fill in the missing details of the derivation of the lower bound of (7.28).

The skeleton inequalities were derived and used to prove the following theorem in [36, 199, 201].

Theorem 7.6. For all $d, \chi(z) \geq \operatorname{const}\left(1-z / z_{c}\right)^{-1 / 2}$ for $0 \leq z \leq z_{c}$. If the square condition is satisfied then the reverse inequality also holds, and hence

$$
\chi(z) \simeq\left(1-z / z_{c}\right)^{-1 / 2} .
$$


Proof. We first discuss the integration of the upper bound of (7.28), to obtain the lower bound in the statement of the theorem. Fix some $z_{0} \in\left(0, z_{c}\right)$. There is no difficulty for $z<z_{0}$. For $z \geq z_{0}$, (7.28) implies that

$$
\frac{\mathrm{d}[z \chi(z)]}{\mathrm{d} z} \leq \frac{1}{z_{0}^{3}}[z \chi(z)]^{3}
$$

and hence

$$
-\frac{\mathrm{d}[z \chi(z)]^{-2}}{\mathrm{~d} z} \leq \frac{2}{z_{0}^{3}} .
$$

Integration of this inequality over the interval $\left[z, z_{c}\right]$ gives

$$
[z \chi(z)]^{-2}-\left[z_{c} \chi\left(z_{c}\right)\right]^{-2} \leq \frac{2}{z_{0}^{3}}\left(z_{c}-z\right) .
$$

If we knew that $\chi\left(z_{c}\right)=\infty$, this would give the desired lower bound on the susceptibility. Since $z_{c}$ is the radius of convergence of $\chi$, we do know that $\chi(z)=\infty$ for $z>z_{c}$, and we need to rule out the possibility of a jump at $z_{c}$.

To do so, we argue as follows. Consider lattice trees which lie in the finite set $\Lambda_{R}$ given by the intersection of $[-R, R]^{d}$ with $\mathbb{Z}^{d}$; the lack of translation invariance of $\Lambda_{R}$ complicates the argument. For $x, y \in \Lambda_{R}$, let

$$
\begin{aligned}
G_{R, z}(x, y) & =\sum_{T \subset \Lambda_{R}: T \ni x, y} z^{|T|}, \\
\chi_{R, x}(z) & =\sum_{y \in \Lambda_{R}} G_{R, z}(x, y), \\
\bar{\chi}_{R}(z) & =\max _{x \in \Lambda_{R}} \chi_{R, x}(z) .
\end{aligned}
$$

Note that

$$
\begin{aligned}
\chi(z) & =\lim _{R \rightarrow \infty} \chi_{R, 0}(z)=\sup _{R} \chi_{R, 0}(z) \\
& =\lim _{R \rightarrow \infty} \bar{\chi}_{R}(z)=\sup _{R} \bar{\chi}_{R}(z) .
\end{aligned}
$$

It is not difficult to follow the steps leading to the upper bound of (7.28) to see that for each $x \in \Lambda_{R}$,

$$
\frac{\mathrm{d}\left[z \chi_{R, x}(z)\right]}{\mathrm{d} z} \leq \chi_{R, x}(z) \bar{\chi}_{R}(z)^{2} \leq \bar{\chi}_{R}(z)^{3} .
$$

Since each $\chi_{R, x}$ is a polynomial, and since there are finitely many $x \in \Lambda_{R}$, for each $z$ there is an $x$ such that the derivative of $\chi_{R, x}$ is equal to the derivative of $\bar{\chi}_{R}$, except possibly for finitely many values of $z$. Apart from this exceptional set, it follows from (7.37) that

$$
-\frac{\mathrm{d}\left[z \bar{\chi}_{R}(z)\right]^{-2}}{\mathrm{~d} z} \leq \frac{2}{z^{3}} .
$$


Each $\chi_{R, x}$ is a polynomial in $z$ which is positive for all $z \geq 0$, and hence $\bar{\chi}_{R}^{-2}$ is continuous on $[0, \infty)$. The bound $(7.38)$ implies that $\left[z \bar{\chi}_{R}(z)\right]^{-2}$ is equicontinuous on $\left[z_{0}, \infty\right)$. By Lemma 5.13 , this implies that

$$
[z \chi(z)]^{-2}=\inf _{R}\left[z \bar{\chi}_{R}(z)\right]^{-2}=-\sup _{R}\left(-\left[z \bar{\chi}_{R}(z)\right]^{-2}\right)
$$

is continuous on $\left[z_{0}, \infty\right)$. Since $\chi(z)^{-2}=0$ for $z>z_{c}$, it follows that $\chi\left(z_{c}\right)^{-2}=$ 0 , as required.

For the upper bound, we will only observe here the weaker result that if $S\left(z_{c}\right)-g_{z_{c}}^{4}<\left(3 g_{z_{c}}^{2}\right)^{-1}$, then the lower bound of (7.30) can be integrated to give the upper bound on $\chi$ stated in the theorem. Since $g_{z_{c}}^{2} \leq S\left(z_{c}\right)$ by definition, the above inequality is implied if $S\left(z_{c}\right)\left(S\left(z_{c}\right)-g_{z_{c}}^{4}\right)<\frac{1}{3}$, which follows if $S\left(z_{c}\right)-g_{z_{c}}^{4}$ is sufficiently small. In applications of the lace expansion, it is always proved that $S\left(z_{c}\right)-g_{z_{c}}^{4}$ is small, so the weaker result suffices in practice. However, the method used in [12], to deal with the analogous issue for percolation, can actually be applied to show that in fact $S\left(z_{c}\right)<\infty$ is sufficient to imply the desired upper bound on the susceptibility [199].

By analogy with the discussion of Section 5.4, it might be expected that for $d>8$ there is a constant $v$ such that

$$
\frac{\mathrm{d}[z \chi(z)]}{\mathrm{d} z} \sim v \chi(z)^{3} \quad \text { as } z \rightarrow z_{c}^{-} .
$$

In fact, the lace expansion has been used to prove that under the hypotheses of Theorem 7.3,

$$
\begin{aligned}
\frac{\mathrm{d}[z \chi(z)]}{\mathrm{d} z} & =\frac{1}{2 \pi A^{2}} \chi(z)^{3}+O\left(\left|1-z / z_{c}\right|^{-3 / 2+\epsilon}\right) \\
& =\frac{A \sqrt{\pi}}{2\left(1-z / z_{c}\right)^{3 / 2}}+O\left(\left|1-z / z_{c}\right|^{-3 / 2+\epsilon}\right)
\end{aligned}
$$

holds uniformly in complex $z$ with $|z|<z_{c}$, where $A$ is the constant of (7.15) and (7.17). The proof of (7.41) in [99] actually uses a double expansion, although the lace expansion on a tree derived in [124] can now be used instead to do it with a single expansion [125].

The asymptotic formula (7.41) implies (7.15) via a Tauberian theorem, as the following exercise shows.

Exercise 7.7. (a) Suppose that $f(z)=\sum_{n=0}^{\infty} a_{n} z^{n}$ has radius of convergence 1. Suppose that $|f(z)| \leq$ const $|1-z|^{-b}$ uniformly in $|z|<1$, with $b \geq 1$. Prove that $\left|a_{n}\right| \leq$ const $n^{b-1}$ if $b>1$, and that $\left|a_{n}\right| \leq$ const $\log n$ if $b=1$. Hint:

$$
a_{n}=\frac{1}{2 \pi i} \oint_{\Gamma} f(z) \frac{\mathrm{d} z}{z^{n+1}}
$$

where $\Gamma$ is the contour $|z|=1-\frac{1}{n}$. (See [75] or [62] for a solution.)

(b) Conclude (7.15) from (7.41). 


\section{The Lace Expansion for Lattice Trees}

In this section, we derive the lace expansion for lattice trees. We also indicate how the expansion can be modified to apply to lattice animals, as this gives some insight into the expansion for percolation to be discussed later in Section 10. Finally, we briefly discuss diagrammatic estimates for lattice trees. The ideas in this section are from [95]. Convergence of the expansion will not be discussed here; see $[95,91,125]$ for three different methods to prove convergence.

\subsection{Expansion for Lattice Trees}

We first discuss the derivation of the expansion using connected graphs and laces, and then briefly indicate how to interpret the expansion as arising from repeated inclusion-exclusion.

Given two distinct vertices $x, y$ and a tree $T \ni x, y$, the backbone is defined to be the unique path, consisting of bonds of $T$, which joins $x$ to $y$. If the backbone consists of $n$ bonds, and is considered to start at $x$ and end at $y$, then removal of the bonds in the backbone disconnects $T$ into $n+1$ mutually nonintersecting trees $R_{0}, \ldots, R_{n}$, which we refer to as ribs. This decomposition is shown in Fig. 8.1.

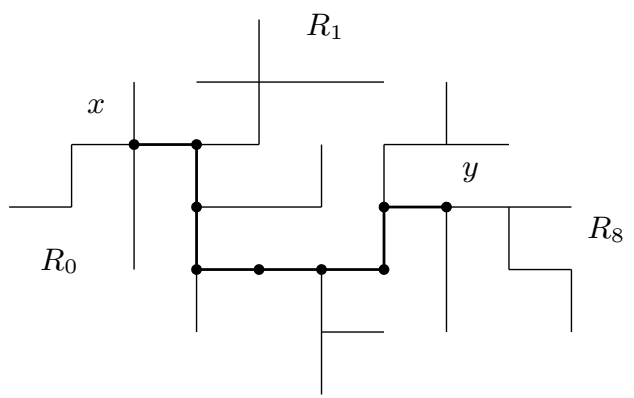

Fig. 8.1. Decomposition of a tree $T$ containing vertices $x$ and $y$ into its backbone and ribs $R_{0}, \ldots, R_{8}$. The vertices of the backbone are indicated by heavy dots.

Given a set $\vec{R}=\left\{R_{0}, \ldots, R_{n}\right\}$ of $n+1$ trees $R_{j}$, we define

$$
U_{s t}(\vec{R})=\left\{\begin{aligned}
-1 & \text { if } R_{s} \text { and } R_{t} \text { share a common vertex } \\
0 & \text { if } R_{s} \text { and } R_{t} \text { share no common vertex. }
\end{aligned}\right.
$$

Then the two-point function can be written

$$
G_{z}(x)=\sum_{\omega \in \mathcal{W}(0, x)} z^{|\omega|} \sum_{R_{0} \ni 0} \cdots \sum_{R_{|\omega|} \ni x} z^{\left|R_{0}\right|+\ldots+\left|R_{|\omega|}\right|} \prod_{0 \leq s<t \leq|\omega|}\left(1+U_{s t}(\vec{R})\right),
$$


where the sum over $\omega$ is over all simple random walks from 0 to $x$, and where each sum over $R_{i}$ is a sum over trees containing $\omega(i)$, and $\vec{R}=\left(R_{0}, \ldots, R_{|\omega|}\right)$. We will abbreviate the right hand side of (8.2) as

$$
G_{z}(x)=\sum_{\omega \in \mathcal{W}(0, x)} z^{|\omega|}\left[\prod_{i=0}^{|\omega|} \sum_{R_{i} \ni \omega(i)} z^{\left|R_{i}\right|}\right] \prod_{0 \leq s<t \leq|\omega|}\left(1+U_{s t}(\vec{R})\right),
$$

with the understanding that the sums over $R_{i}$ cannot be completed in (8.3) without taking into account the product over $s, t$. The identity (8.2) represents the generating function for lattice trees containing 0 and $x$ by a sum which generates backbones $\omega$ and ribs $\vec{R}$, with an interaction to prevent the ribs from intersecting each other. The sum over $\omega$ is the sum over all random walks from 0 to $x$ (taking steps in $\Omega$ ), although walks that are not self-avoiding give zero contribution to (8.2).

\subsubsection{The Expansion}

We use the terminology of Definitions 3.2 and 3.5, but with a change in the definition of connected graph. A graph $\Gamma$ on $[a, b]$ is now be said to be connected if both $a$ and $b$ are endpoints of edges in $\Gamma$, and if in addition for each $c \in(a, b)$ there are $s, t \in[a, b]$ such that $s<c<t$ with either (i) $\{s, t\} \in \Gamma$, or (ii) $\{c, t\} \in \Gamma$ and $\{s, c\} \in \Gamma$. Equivalently, $\Gamma$ is connected if $\cup_{s t \in \Gamma}[s, t]=[a, b]$. This notion of connectedness is less restrictive than that used for the self-avoiding walk, and is better suited for dealing with the interaction between ribs. This new definition of connected graph leads to a larger set of laces than before, where we still define a lace to be a minimally connected graph, i.e., a connected graph for which the removal of any edge would result in a disconnected graph. The set of laces on $[a, b]$ is denoted $\mathcal{L}[a, b]$. We also modify the prescription associating to each connected graph $\Gamma$ a unique lace $\mathrm{L}_{\Gamma}$, to conform with the new notion of connectedness. Namely, we now define $\mathrm{L}_{\Gamma}$ to consist of edges $s_{1} t_{1}, s_{2} t_{2}, \ldots$, with $t_{1}, s_{1}, t_{2}, s_{2}, \ldots$ determined, in that order, by

$$
\begin{aligned}
t_{1}=\max \{t: a t \in \Gamma\}, & s_{1}=a, \\
t_{i+1}=\max \left\{t: \exists s \leq t_{i} \text { such that } s t \in \Gamma\right\}, & s_{i+1}=\min \left\{s: s t_{i+1} \in \Gamma\right\} .
\end{aligned}
$$

As in Section 3.3, given a lace $L$, we define $\mathcal{C}(L)=\left\{s t \notin L:\left.\mathrm{L}\right|_{L \cup\{s t\}}=L\right\}$.

Let

$$
K[a, b]=\prod_{a \leq s<t \leq b}\left(1+U_{s t}\right),
$$

and, as in (3.9) and (3.19),

$$
\begin{aligned}
J[0, a] & =\sum_{\Gamma \in \mathcal{G}[a, b]} \prod_{s t \in \Gamma} U_{s t} \\
& =\sum_{L \in \mathcal{L}[0, a]} \prod_{s t \in L} U_{s t} \prod_{s^{\prime} t^{\prime} \in \mathcal{C}(L)}\left(1+U_{s^{\prime} t^{\prime}}\right),
\end{aligned}
$$


where now $\mathcal{G}[a, b]$ is the set of connected graphs with the new definition of connectivity.

Exercise 8.1. Modify the proof of Lemma 3.4 to show that with the new definition of connectivity,

$$
K[0, b]=K[1, b]+\sum_{a=1}^{b-1} J[0, a] K[a+1, b]+J[0, b],
$$

for $b \geq 1$ (the empty sum is 0 if $b=1$ ).

Substitution of (8.6) into (8.3) results in

$$
\begin{aligned}
G_{z}(x)= & \sum_{R_{0} \ni 0} z^{\left|R_{0}\right|} \delta_{0, x}+\sum_{\substack{\omega \in \mathcal{W}(0, x) \\
|\omega| \geq 1}} z^{|\omega|}\left[\prod_{i=0}^{|\omega|} \sum_{R_{i} \ni \omega(i)} z^{\left|R_{i}\right|}\right] K[1,|\omega|] \\
& +\sum_{\substack{\omega \in \mathcal{W}(0, x) \\
|\omega| \geq 2}} z^{|\omega|}\left[\prod_{i=0}^{[\omega \mid} \sum_{R_{i} \ni \omega(i)} z^{\left|R_{i}\right|}\right] \sum_{a=1}^{|\omega|-1} J[0, a] K[a+1,|\omega|] \\
& +\sum_{\substack{\omega \in \mathcal{W}(0, x) \\
|\omega| \geq 1}} z^{|\omega|}\left[\prod_{i=0}^{|\omega|} \sum_{R_{i} \ni \omega(i)} z^{\left|R_{i}\right|}\right] J[0,|\omega|] .
\end{aligned}
$$

The first term on the right hand side is due to the contribution to (8.3) from the zero-step walk, and the other terms are due to the walks $\omega$ with $|\omega| \geq 1$.

Recalling our notation $g_{z}$ for the one-point function, and writing

$$
\Pi_{z}(x)=\sum_{\substack{\omega \in \mathcal{W}(0, x) \\|\omega| \geq 1}} z^{|\omega|}\left[\prod_{i=0}^{|\omega|} \sum_{R_{i} \ni \omega(i)} z^{\left|R_{i}\right|}\right] J[0,|\omega|],
$$

the first and last terms on the right hand side of (8.7) are equal to $g_{z} \delta_{0, x}$ and $\Pi_{z}(x)$, respectively. The second term on the right hand side of (8.7) is equal to

$$
\sum_{R_{0} \ni 0} z^{\left|R_{0}\right|} z \sum_{u \in \Omega} G_{z}(x-u)=g_{z}\left(z|\Omega| D * G_{z}\right)(x),
$$

where $D$ is defined by (1.10). For the third term on the right hand side of (8.7), we consider $\omega$ to be composed of an initial $a$-step walk $\omega_{1}$ from 0 to (say) $u$, followed by a single step to (say) $v$, and then a final portion $\omega_{2}$ from $v$ to $x$. The term in question is then equal to 


$$
\begin{gathered}
z \sum_{(u, v)} \sum_{\substack{\omega_{1} \in \mathcal{W}(0, u) \\
\left|\omega_{1}\right| \geq 1}} z^{\left|\omega_{1}\right|}\left[\prod_{i=0}^{\left|\omega_{1}\right|} \sum_{R_{i} \ni \omega_{1}(i)} z^{\left|R_{i}\right|}\right] J\left[0,\left|\omega_{1}\right|\right] \\
\times \sum_{\substack{\omega_{2} \in \mathcal{W}(v, x) \\
\left|\omega_{2}\right| \geq 0}} z^{\left|\omega_{2}\right|}\left[\prod_{i=0}^{\left|\omega_{2}\right|} \sum_{R_{i} \ni \omega_{2}(i)} z^{\left|R_{i}\right|}\right] K\left[0,\left|\omega_{2}\right|\right] \\
=z \sum_{(u, v)} \Pi_{z}(u) G_{z}(x-v)=\left(\Pi_{z} * z|\Omega| D * G_{z}\right)(x),
\end{gathered}
$$

where the sum over $(u, v)$ denotes the sum over all directed steps with $v-u \in$ $\Omega$.

Summarizing, (8.7) can be rewritten as

$$
G_{z}(x)=g_{z} \delta_{0, x}+\Pi_{z}(x)+g_{z}\left(z|\Omega| D * G_{z}\right)(x)+\left(\Pi_{z} * z|\Omega| D * G_{z}\right)(x) .
$$

This is the lace expansion for lattice trees. We can write this more compactly by defining

$$
h_{z}(x)=g_{z} \delta_{0, x}+\Pi_{z}(x) .
$$

Then (8.11) becomes

$$
G_{z}(x)=h_{z}(x)+\left(h_{z} * z|\Omega| D * G_{z}\right)(x) .
$$

It is sometimes useful to modify the above analysis by introducing a "fugacity" $\zeta$ associated to backbone length. Thus, we define

$$
G_{z, \zeta}(x)=\sum_{T: T \ni 0, x} z^{|T|} \zeta^{\left|\omega_{T}(x)\right|},
$$

where $\omega_{T}(x)$ denotes the backbone joining 0 to $x$ in $T$. This is the same as inserting a factor $\zeta^{|\omega|}$ in (8.3). Following the steps of the expansion, we obtain

$$
G_{z, \zeta}(x)=h_{z, \zeta}(x)+\left(h_{z, \zeta} * z \zeta|\Omega| D * G_{z, \zeta}\right)(x),
$$

where

$$
h_{z, \zeta}(x)=g_{z} \delta_{0, x}+\Pi_{z, \zeta}(x)
$$

with $\Pi_{z, \zeta}$ given by (8.8) with an additional factor $\zeta^{|\omega|}$ inserted in the right hand side.

\subsubsection{Inclusion-Exclusion}

We now interpret the expansion (8.11) as the result of repeated inclusionexclusion.

The case $|\omega|=0$ in (8.2) gives rise to the term $g_{z} \delta_{0, x}$ in (8.11). Consider the case $|\omega| \geq 1$, and suppose that in (8.2) we neglect the fact that the rib 
$R_{0}$ at the origin should avoid the other ribs. This is the same as replacing the interaction $K[0,|\omega|]$ by $K[1,|\omega|]$. The result can be factorized, and produces the term $g_{z}\left(z|\Omega| D * G_{z}\right)(x)$ on the right hand side of (8.11).

In neglecting the fact that $R_{0}$ should avoid the other ribs, we have included terms in which a rib $R_{j}$ with $j \geq 1$ intersects $R_{0}$, and we should exclude their contribution by subtracting a correction term. Given a configuration in which some $R_{j}$ with $j \geq 1$ intersects $R_{0}$, let $j_{0}$ be the smallest value of $j$ for which $R_{j}$ intersects $R_{0}$. As a first approximation, we neglect the fact that the ribs $R_{j}$ with $j>j_{0}$ should avoid those with $1 \leq j \leq j_{0}$. In this approximation, the subtracted term is the convolution with $\left(z|\Omega| D * G_{z}\right)$ of a generating function for tree-like configurations in which the first and last ribs intersect each other. To correct for the approximation, a term involving further rib intersections must be added, and so on. This leads to an identity of the form (8.11), in which $\Pi_{z}$ is represented by an alternating series. This alternating series will appear in more detail when diagrammatic estimates are discussed in Section 8.3.

\subsection{Expansion for Lattice Animals}

A lattice animal on $\mathbb{Z}^{d}$ is defined to be a finite connected set of bonds, which may or may not contain cycles. Lattice animals are predicted to have the same critical exponents and scaling limit as lattice trees.

The lace expansion for lattice trees can be modified to apply to lattice animals. The main difference compared to lattice trees is that a lattice animal containing vertices $x$ and $y$ need not contain a unique path joining $x$ and $y$. To deal with this, some definitions are needed.

A lattice animal $A$ containing $x$ and $y$ is said to have a double connection from $x$ to $y$ if there are two bond-disjoint self-avoiding walks in $A$ between $x$ and $y$ (the walks may share a common vertex, but no common bond), or if $x=y$. A bond $\{u, v\}$ in $A$ is called pivotal for the connection from $x$ to $y$ if its removal would disconnect the animal into two connected components with $x$ in one connected component and $y$ in the other. There is a natural order to the set of pivotal bonds for the connection from $x$ to $y$, and each pivotal bond is ordered in a natural way, as follows. The first pivotal bond for the connection from $x$ to $y$ (assuming there is at least one) is the pivotal bond for which there is a double connection between one endpoint of the pivotal bond and $x$. The endpoint for which there is a double connection to $x$ is then the first endpoint of the first pivotal bond. To determine the second pivotal bond, the role of $x$ is then played by the second endpoint of the first pivotal bond, and so on.

Let

$$
G_{z}^{a}(x)=\sum_{A: A \ni 0, x} z^{|A|}
$$

denote the two-point function for lattice animals, where $|A|$ is the number of bonds in $A$. Given two vertices $x, y$ and an animal $A$ containing $x$ and $y$, the 


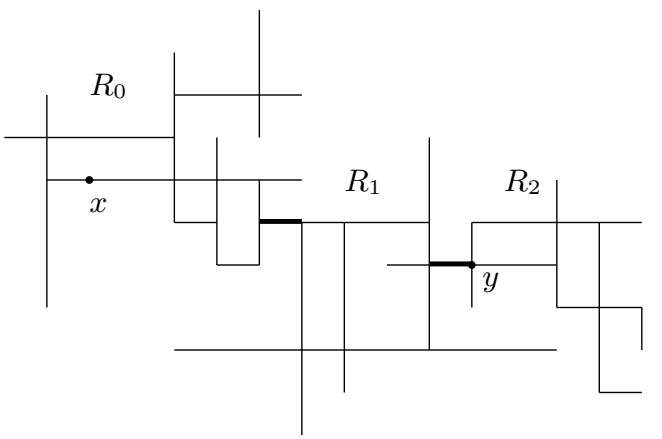

Fig. 8.2. Decomposition of a lattice animal $A$ containing $x$ and $y$ into backbone and ribs. The backbone, consisting of two bonds, is drawn in bold lines.

backbone of $A$ is now defined to be the set of pivotal bonds for the connection from $x$ to $y$. In general this backbone is not connected. The ribs of $A$ are the connected components which remain after the removal of the backbone from A. An example is depicted in Fig. 8.2. The set of all animals having a double connection between $x$ and $y$ is denoted $\mathcal{D}_{x, y}$, and we write

$$
g_{z}^{a}(y-x)=\sum_{R \in \mathcal{D}_{x, y}} z^{|R|} .
$$

In particular $\mathcal{D}_{x, x}$ is the set of all animals containing $x$. Let $B$ be an arbitrary finite ordered set of directed bonds:

$$
B=\left(\left(u_{1}, v_{1}\right), \ldots,\left(u_{|B|}, v_{|B|}\right)\right) .
$$

Let $v_{0}=0$ and $u_{|B|+1}=x$. Then

$$
G_{z}^{a}(x)=\sum_{B:|B| \geq 0} z^{|B|}\left[\prod_{i=0}^{|B|} \sum_{R_{i} \in \mathcal{D}_{v_{i}, u_{i+1}}} z^{\left|R_{i}\right|}\right] K[0,|B|],
$$

where $K[0,|B|]$ is defined in (8.4), with $U_{s t}$ of $(8.1)$.

Recall the definition of $J[0, a]$ in (8.5), and define

$$
\Pi_{z}^{a}(y)=\sum_{B:|B| \geq 1} z^{|B|}\left[\prod_{i=0}^{|B|} \sum_{R_{i} \in \mathcal{D}_{v_{i}, u_{i+1}}} z^{\left|R_{i}\right|}\right] J[0,|B|]
$$

and

$$
h_{z}^{a}(x)=g_{z}^{a}(x)+\Pi_{z}^{a}(x) .
$$

A calculation similar to that used to derive (8.11), using (8.6), gives

$$
G_{z}^{a}(x)=h_{z}^{a}(x)+\left(h_{z}^{a} * z|\Omega| D * G_{z}^{a}\right)(x) .
$$

This is the lace expansion for lattice animals. 


\subsection{Diagrammatic Estimates for Lattice Trees}

To prove convergence of the lace expansion for lattice trees, bounds are required on $\hat{\Pi}_{z}(k)$ and $\hat{\Pi}_{z}(0)-\hat{\Pi}_{z}(k)$. In this section we sketch the idea of how appropriate diagrammatic bounds can be obtained on $\hat{\Pi}_{z}(k)$. The procedure for obtaining bounds for lattice animals is similar but more involved, and will not be discussed here. For further details on diagrammatic estimates, both for lattice trees and lattice animals, see [95, 91].

We will not discuss the actual convergence proof here. One approach to convergence, based on Lemma 5.9 and related in spirit to the proof for the selfavoiding walk given in Section 5.2, can be found in [95]. A second approach, also based on Lemma 5.9 , works directly in $x$-space and does not use Fourier transforms [91]. A third approach, based on the induction method of [120], is given in [125].

For the diagrammatic estimates for lattice trees, we recall the (new) notions of connectivity and lace discussed in Section 8.1.1. We take $U_{s t}$ to be defined by $(8.1)$, and we let $\mathcal{L}^{(N)}[a, b]$ denote the set of laces in $\mathcal{L}[a, b]$ consisting of exactly $N$ edges. Let

$$
J^{(N)}[a, b]=\sum_{L \in \mathcal{L}^{(N)}[a, b]} \prod_{s t \in L} U_{s t} \prod_{s^{\prime} t^{\prime} \in \mathcal{C}(L)}\left(1+U_{s^{\prime} t^{\prime}}\right)
$$

and

$$
\Pi_{z}^{(N)}(x)=(-1)^{N} \sum_{\substack{\omega \in \mathcal{W}(0, x) \\|\omega| \geq 1}} z^{|\omega|}\left[\prod_{i=0}^{|\omega|} \sum_{R_{i} \ni \omega(i)} z^{\left|R_{i}\right|}\right] J^{(N)}[0,|\omega|] .
$$

By definition, $\Pi_{z}^{(N)}(x)$ is non-negative. By (8.8) and (8.5),

$$
\Pi_{z}(x)=\sum_{N=1}^{\infty}(-1)^{N} \Pi_{z}^{(N)}(x)
$$

To state the diagrammatic estimates, we make the following definitions. We extract the term in the definition (7.26) of the square diagram due to $x=y=u=0$ and define a generalized square diagram by

$$
\overline{\mathrm{S}}(z)=\sup _{w \in Z^{d}}\left[\sum_{x, y, u} G_{z}(x) G_{z}(y-x) G_{z}(u-y) G_{z}(w-u)-\delta_{0, w} g_{z}^{4}\right] .
$$

We also define a generalized triangle diagram

$$
\overline{\mathbf{T}}(z)=\sup _{u \in Z^{d}}\left[\sum_{x, y} G_{z}(x) G_{z}(y-x) G_{z}(u-y)-\delta_{0, u} g_{z}^{3}\right] .
$$


Theorem 8.2. For $z \geq 0$ and $N \geq 1$,

$$
\sum_{x \in \mathbb{Z}^{d}} \Pi_{z}^{(N)}(x) \leq \overline{\mathrm{T}}(z)(2 \overline{\mathrm{S}}(z))^{N-1} .
$$

Proof. We discuss the proof in detail only for the case $N=1$, and sketch the proof for $N \geq 2$.

For $N=1$, there is a unique lace consisting of a single edge, and all other bonds are compatible with this lace. Therefore, by (8.22),

$$
J^{(1)}[a, b]=U_{a b} \prod_{\substack{a \leq s<t \leq b \\(s, t) \neq(a, b)}}\left(1+U_{s t}\right)
$$

and (8.23) then gives

$$
\Pi_{z}^{(1)}(x)=\sum_{\substack{\omega \in \mathcal{W}(0, x) \\|\omega| \geq 1}} z^{|\omega|}\left[\prod_{i=0}^{|\omega|} \sum_{R_{i} \ni \omega(i)} z^{\left|R_{i}\right|}\right]\left(-U_{0|\omega|}\right) \prod_{\substack{0 \leq s<t \leq|\omega| \\ s, t) \neq(0,|\omega|)}}\left(1+U_{s t}\right) .
$$

The factor $-U_{0|\omega|}$ gives a nonzero contribution only if $R_{0}$ and $R_{|\omega|}$ intersect, and the final product in (8.29) disallows any further rib intersections. We first consider the case $x \neq 0$. Relaxing the latter restriction somewhat and overcounting an enforcement of the former gives the upper bound

$$
\begin{aligned}
\Pi_{z}^{(1)}(x) \leq & \sum_{v \in \mathbb{Z}^{d}} \sum_{\substack{\omega \in \mathcal{W}(0, x) \\
|\omega| \geq 1}} z^{|\omega|} \sum_{R_{0} \ni 0, v}|z|^{\left|R_{0}\right|} \sum_{R_{|\omega|} \ni x, v} z^{\left|R_{|\omega|}\right|} \\
& \times\left[\prod_{i=1}^{[\omega \mid-1} \sum_{R_{i} \ni \omega(i), \not 0, x} z^{\left|R_{i}\right|}\right] \prod_{1 \leq s<t \leq|\omega|-1}\left(1+U_{s t}\right) .
\end{aligned}
$$

Since

$$
\sum_{R_{0} \ni 0, v} z^{\left|R_{0}\right|}=G_{z}(v), \quad \sum_{R_{|\omega|} \ni x, v} z^{\left|R_{|\omega|}\right|}=G_{z}(v-x),
$$

and since

$$
\sum_{\substack{\omega \in \mathcal{W}(0, x) \\|\omega| \geq 1}} z^{|\omega|}\left[\prod_{i=1}^{|\omega|-1} \sum_{R_{i} \ni \omega(i), \not \supset 0, x} z^{\left|R_{i}\right|}\right] \prod_{1 \leq s<t \leq|\omega|-1}\left(1+U_{s t}\right) \leq G_{z}(x),
$$

for $x \neq 0$ we have

$$
\Pi_{z}^{(1)}(x) \leq \sum_{v \in \mathbb{Z}^{d}} G_{z}(x) G_{z}(v-x) G_{z}(v) .
$$


When $x=0$ in (8.29), we can argue using similar ideas that

$$
\Pi_{z}^{(1)}(0) \leq \sum_{v \in \Omega} G_{z}(v)^{2}
$$

by neglecting the interaction between the last rib and all previous ribs. In particular, the term $v=0$ is not present in the above sum. Since $G_{z}(0)=$ $g_{z} \geq 1$, we can combine (8.33) and (8.34) to obtain

$$
\sum_{x \in \mathbb{Z}^{d}} \Pi_{z}^{(1)}(x) \leq \sum_{x, v \in \mathbb{Z}^{d}} G_{z}(v) G_{z}(x-v) G_{z}(x)-g_{z}^{3} \leq \overline{\mathbf{T}}(z) .
$$

This gives the desired result for $N=1$. Note that the first upper bound in (8.35) is just the $u=0$ term of the supremum in (8.26).

A similar strategy can be used to bound $\Pi_{z}^{(N)}(x)$ for $N \geq 2$, and we sketch the argument. The situation for $N=2$ is shown in Fig. 8.3. For a lace $L=\left(0 t_{1}, s_{2}|\omega|\right)$ consisting of exactly two edges, there are two generic configurations possible with $\prod_{s t \in L} U_{s t} \neq 0$, one with $s_{2}=t_{1}$ and one with $s_{2}<t_{1}$.

We illustrate the method for the former case. The contribution to $\Pi_{z}^{(2)}$ due to laces with $s_{2}=t_{1}$ can be written

$$
\sum_{\substack{\omega \in \mathcal{W}(0, x) \\|\omega| \geq 2}} z^{|\omega|}\left[\prod_{i=0}^{|\omega|} \sum_{R_{i} \ni \omega(i)} z^{\left|R_{i}\right|}\right] \sum_{s=1}^{|\omega|-1} U_{0 s} U_{s|\omega|} \prod_{s^{\prime} t^{\prime} \in \mathcal{C}(0 s, s|\omega|)}\left(1+U_{s^{\prime} t^{\prime}}\right) .
$$

For $U_{0 s} \neq 0, R_{0}$ and $R_{s}$ must intersect. Let $y$ be a vertex where $R_{0}$ and $R_{s}$ intersect, and let $\omega_{R_{s}}$ be the backbone of $R_{s}$ joining $\omega(s)$ and $y$. For $U_{s|\omega|} \neq 0$, $R_{|\omega|}$ must intersect $R_{s}$, and hence there must be a rib emanating from a vertex on the backbone $\omega_{R_{s}}$ which intersects $R_{|\omega|}$ - see Fig. 8.3. By neglecting some of the rib avoidance conditions, and by arguing in a similar fashion to the case $N=1,(8.36)$ can be bounded above by the Feynman diagram depicted at upper right in Fig. 8.3. The contribution from the other type of lace is bounded by the Feynman diagram at lower right in Fig. 8.3. In evaluating the diagrams, sums over vertices are constrained to disallow the coincidence of all vertices on any loop.

Explicitly, the upper bound is

$$
\begin{aligned}
\sum_{x \in \mathbb{Z}^{d}} \Pi_{z}^{(2)}(x) \leq 2 \sum & G_{z}(u) G_{z}(v) G_{z}(w-u) G_{z}(w-v) \\
\times & G_{z}(x-w) G_{z}(y-u) G_{z}(x-y),
\end{aligned}
$$

where the sum is taken over all $u, v, w, x, y$ except the terms $u=v=w=0$ and $v=w=x=y$. We estimate this by taking the supremum over $u, w$ of the sum over $x, y$ of the second line, and obtain the desired upper bound $2 \overline{\mathrm{S}}(z) \overline{\mathrm{T}}(z)$. 

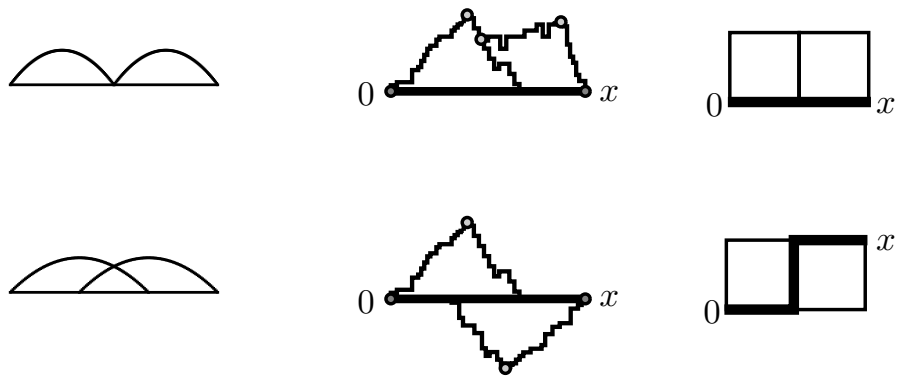

Fig. 8.3. The two generic laces consisting of two bonds, schematic diagrams showing the corresponding rib intersections for a nonzero contribution to $\Pi_{z}^{(2)}(x)$, and Feynman diagrams bounding the corresponding contributions to $\Pi_{z}^{(2)}(x)$. Diagram lines corresponding to the backbone joining 0 and $x$ are shown in bold.

In general, $\Pi_{z}^{(N)}(x)$ is bounded above by a sum of $2^{N-1}$ "ladder" diagrams, each containing $N$ non-trivial loops. The diagrams are shown in Fig. 8.4 for $N=3$. For general $N$, the diagrams can be estimated inductively to prove the desired upper bound. See [95] for details.

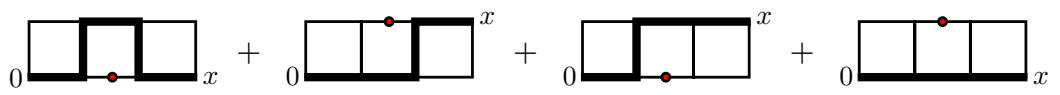

Fig. 8.4. The Feynman diagrams bounding $\Pi_{z}^{(N)}(x)$ for $N=3$. Diagram lines corresponding to the backbone joining 0 and $x$ are shown in bold.

Exercise 8.3. (a) Show that the diagrams depicted in Fig. 8.4 are correct.

(b) Determine the diagrams for $N=4$.

\section{Percolation}

This is the first of several sections that pertain to percolation. In the present section, we define the model and discuss the phase transition, and then consider several differential inequalities that are relevant for the critical behaviour. The discussion is similar to that of Section 2 for the self-avoiding walk and the bubble condition, and to that of Section 7 for lattice trees and the square condition. However, now it is the triangle condition that is relevant, and the differential inequalities for percolation have a richer structure than those for the other models. 
Looking ahead, in Section 10 we derive the lace expansion for percolation and discuss its bounds. In Section 11, we survey results that have been proved for percolation using the expansion. In Sections 12-13, we extend these ideas to oriented percolation, in which bonds are directed in the direction of increasing "time." Then in Section 14, we discuss applications of the expansion to the contact process, for which the time variable is continuous.

\subsection{The Phase Transition}

For an introduction to percolation, see [85]. Introductory material about percolation can also be found in [128], and [197] provides an introduction from a physicist's perspective.

We consider independent Bernoulli bond percolation on the integer lattice $\mathbb{Z}^{d}$, with edge (bond) set consisting of pairs $\{x, y\}$ of vertices of $\mathbb{Z}^{d}$ with $y-x \in \Omega$, where $\Omega$ defines either the nearest-neighbour model (1.1) or the spread-out model (1.2). This means that to each bond $\{x, y\}$ we associate an independent Bernoulli random variable $n_{\{x, y\}}$ which takes the value 1 with probability $p$ and the value 0 with probability $1-p$, where $p$ is a parameter in the closed interval $[0,1]$. If $n_{\{x, y\}}=1$ then we say that the bond $\{x, y\}$ is occupied, and otherwise we say that it is vacant. A configuration is a realization of the random variables for all bonds. The joint probability distribution of the bond variables is denoted $\mathbb{P}_{p}$, with corresponding expectation $\mathbb{E}_{p}$.
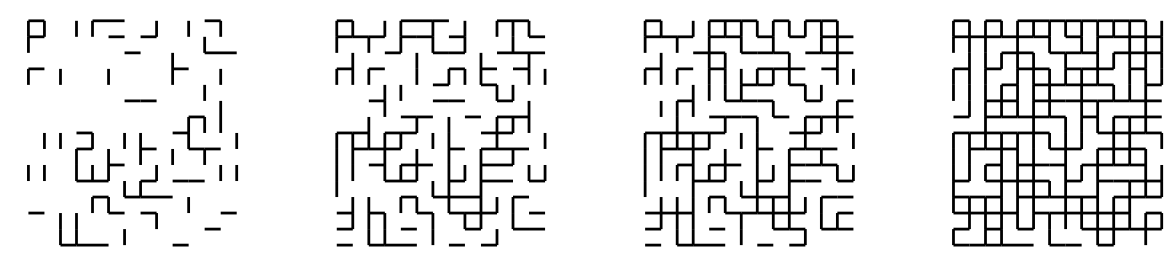

Fig. 9.1. Bond percolation configurations on a $14 \times 14$ piece of the square lattice $\mathbb{Z}^{2}$ for $p=0.25, p=0.45, p=0.55, p=0.75$. The critical value is $p_{c}=\frac{1}{2}$.

Given a configuration and any two vertices $x$ and $y$, we say that $x$ and $y$ are connected, denoted $x \leftrightarrow y$, if there is a path from $x$ to $y$ consisting of occupied bonds, or if $x=y$. We denote by $C(x)$ the random set of vertices connected to $x$, and denote its cardinality by $|C(x)|$. Let

$$
\theta(p)=\mathbb{P}_{p}(|C(0)|=\infty) .
$$

The most basic fact concerning percolation is that there is a phase transition for dimensions $d \geq 2$. That is, for $d \geq 2$, there exists a critical value $p_{c}=$ $p_{c}(d) \in(0,1)$ such that the probability $\theta(p)$ that the origin is connected to infinitely many vertices is zero for $p<p_{c}$, but is strictly positive for $p>p_{c}$. 
It is known that $\theta\left(p_{c}\right)$ is zero for certain two-dimensional models [141], and in high dimensions as discussed further in Section 11.1. It remains an open problem to prove that $\theta\left(p_{c}\right)$ is zero in general dimensions, even though it has been proved that there can be no infinite cluster in any half-space of $\mathbb{Z}^{d}$ when $p=p_{c}[85$, Section 7.3].

The two-point function $\tau_{p}(x)$ is defined to be the probability that 0 and $x$ are connected:

$$
\tau_{p}(x)=\mathbb{P}_{p}(0 \leftrightarrow x) .
$$

By the translation invariance of the lattice, $\mathbb{P}_{p}(x \leftrightarrow y)=\tau_{p}(y-x)$. We will sometimes find it convenient to write the two-point function as

$$
\tau_{p}(x, y)=\tau_{p}(y-x) .
$$

For $p<p_{c}$ the two-point function is known to decay exponentially as $|x| \rightarrow \infty$, so that the correlation length

$$
\xi(p)=-\left[\lim _{n \rightarrow \infty} \frac{1}{n} \log \tau_{p}\left(n e_{1}\right)\right]^{-1},
$$

where $e_{1}$ is the unit vector $(1, \ldots, 0)$, is finite and strictly positive. The susceptibility, or expected cluster size, is defined by

$$
\chi(p)=\sum_{x \in \mathbb{Z}^{d}} \tau_{p}(x)=\mathbb{E}_{p}|C(0)| .
$$

An important theorem [7, 161, 162] asserts that the critical point is also characterized by

$$
p_{c}=\sup \{p: \chi(p)<\infty\} ;
$$

see Exercise 9.6 below.

Let

$$
P_{n}(p)=\mathbb{P}_{p}(|C(0)|=n) .
$$

The magnetization is defined, for $\gamma \in[0,1]$ by $^{8}$

$$
M(p, \gamma)=1-\sum_{n=1}^{\infty}(1-\gamma)^{n} P_{n}(p) .
$$

Note that

$$
M(p, 0)=\theta(p) .
$$

The magnetization has a useful and standard probabilistic interpretation. We define i.i.d. vertex variables taking the value "green" and "not green" by declaring that each vertex is independently green with probability $\gamma \in[0,1]$. The vertex variables are independent of the bond variables. Let $\mathcal{G}$ denote the

\footnotetext{
${ }^{8}$ This $\gamma$ should not be confused with the critical exponent of (9.12), which is denoted by the same Greek letter.
} 
random set of green vertices. Then, for $\gamma \neq 0$, it follows from the definition of the magnetization that

$$
M(p, \gamma)=\mathbb{P}_{p, \gamma}(0 \leftrightarrow \mathcal{G}),
$$

where $\{0 \leftrightarrow \mathcal{G}\}$ denotes the event that $0 \leftrightarrow x$ for some $x \in \mathcal{G}$, and $\mathbb{P}_{p, \gamma}$ denotes the joint distribution of the bond and vertex variables. Note that the limit of $M(p, \gamma)$, as $\gamma \rightarrow 0^{+}$, is equal to $M(p, 0)=\theta(p)$, which is not the same as $\mathbb{P}_{p, 0}(0 \leftrightarrow \mathcal{G})=0$ for $p>p_{c}$.

Another quantity of interest is the number of clusters per vertex, defined by

$$
\kappa(p)=\mathbb{E}_{p}\left(|C(0)|^{-1}\right)=\sum_{n=1}^{\infty} \frac{1}{n} P_{n}(p) .
$$

Various critical exponents are predicted to describe the behaviour of the above functions in the vicinity of the critical point. The critical exponents are often assumed by mathematicians to exist in a rather weak sense, which presumably would be easier to prove. However, it is worth emphasizing that the exponents are actually predicted to exist in an asymptotic sense, as indicated in the following. We use $c$ to denote a positive constant whose value is unimportant and may change from line to line.

On the subcritical side, the following power laws are believed to hold:

$$
\begin{aligned}
\chi(p) & \sim c\left(p_{c}-p\right)^{-\gamma} \quad \text { as } p \rightarrow p_{c}^{-}, \\
\xi(p) & \sim c\left(p_{c}-p\right)^{-\nu} \quad \text { as } p \rightarrow p_{c}^{-}, \\
\kappa^{\prime \prime \prime}(p) & \sim c\left(p_{c}-p\right)^{-1-\alpha} \quad \text { as } p \rightarrow p_{c}^{-}, \\
\frac{\mathbb{E}_{p}\left(|C(0)|^{m+1}\right)}{\mathbb{E}_{p}\left(|C(0)|^{m}\right)} & \sim c\left(p_{c}-p\right)^{-\Delta} \quad \text { as } p \rightarrow p_{c}^{-}, \text {for } m=1,2, \ldots,
\end{aligned}
$$

for some dimension-dependent amplitudes $c$ and critical exponents $\gamma, \nu, \alpha$, $\Delta$. For the spread-out model, the amplitudes $c$ may depend on $L$, but it is believed that the critical exponents are independent of $L$, as long as $L$ is finite.

At the critical point, the following power laws are believed to hold:

$$
\begin{aligned}
\tau_{p_{c}}(x) & \sim c \frac{1}{|x|^{d-2+\eta}} \quad \text { as }|x| \rightarrow \infty, \\
\hat{\tau}_{p_{c}}(k) & \sim c \frac{1}{|k|^{2-\eta}} \quad \text { as } k \rightarrow 0, \\
M\left(p_{c}, \gamma\right) & \sim c \gamma^{1 / \delta} \quad \text { as } \gamma \rightarrow 0, \\
P_{n}\left(p_{c}\right) & \sim c \frac{1}{n^{1+1 / \delta}} \quad \text { as } n \rightarrow \infty,
\end{aligned}
$$

for critical exponents $\eta, \delta$. Finally, on the supercritical side, it is believed that

$$
\theta(p) \sim c\left(p-p_{c}\right)^{\beta} \quad \text { as } p \rightarrow p_{c}^{+},
$$


for a critical exponent $\beta$. Further exponents can also be defined, e.g. the subcritical exponents have supercritical counterparts believed to take on the same values, but we restrict attention to those above, since these are the ones that will figure in the results discussed below.

Important recent advances, connected with the Schramm-Loewner evolutions, have led to proofs of existence of most critical exponents, in a weaker form than the above asymptotic form, for site percolation on the 2-dimensional triangular lattice (see $[190,191,206]$ and references therein).

The lace expansion has been used to prove that the critical exponents exist and take their mean-field values in high dimensions. The mean-field values are the values assumed by the critical exponents for percolation on an infinite regular tree, namely

$$
\begin{gathered}
\gamma=1, \quad \nu=\frac{1}{2}, \quad \alpha=-1, \quad \Delta=2, \\
\eta=0, \quad \delta=2, \quad \beta=1 .
\end{gathered}
$$

A derivation of these exponents for the tree can be found in [85, Section 10.1].

Exercise 9.1. The infinite binary tree is the infinite labelled tree in which every vertex has degree 3 except a root of degree 2 . Consider bond percolation on the infinite binary tree.

(a) Let $\theta(p)$ be the probability that the root is in an infinite cluster. Note that $\eta=1-\theta(p)$ is the extinction probability of the Galton-Watson branching process starting from a single individual, whose offspring distribution $X$ is binomial with parameters 2 and $p$. Thus $\eta$ is the smallest non-negative root of $G(s)=s$, where $G(s)=\mathbb{E} s^{X}$ is the generating function for $X$ (see, e.g., $\left[87\right.$, p. 173]). Conclude that $p_{c}=\frac{1}{2}$ and

$$
\theta(p)= \begin{cases}0 & \text { if } p \leq \frac{1}{2} \\ \frac{1}{p^{2}}(2 p-1) & \text { if } p \geq \frac{1}{2}\end{cases}
$$

so that $\beta=1$.

(b) By conditioning on the number of occupied bonds containing the root, prove that $\chi(p)=(1-2 p)^{-1}$ and hence $\gamma=1$.

It was first predicted in [202] that the upper critical dimension for percolation is 6 . The lace expansion has been applied to spread-out percolation models in dimensions $d>6$, and to the nearest-neighbour model in higher dimensions (often $d \geq 19$ is high enough). These results show that the upper critical dimension is at most 6 . On the other hand, it is known from the results of $[50,200]$ (see also [34]) that the upper critical dimension of percolation is at least six. In brief, these authors proved hyperscaling inequalities, such as $d \nu \geq 2 \Delta-\gamma$, assuming the exponents exist. Inserting the mean-field values $\gamma=1, \Delta=2, \nu=\frac{1}{2}$, we see that for $d<6$ the inequality is not satisfied and hence at least one of these exponents cannot take on its mean-field value. 


\subsection{Differential Inequalities}

One-sided mean-field bounds for the critical exponents $\gamma, \beta$ and $\delta$, valid in all dimensions $d \geq 2$ can be derived from differential inequalities for the susceptibility and the magnetization. The first of these differential inequalities, for $\chi$, is summarized in the following proposition, which is due to [12]. The proof of the proposition is deferred to Section 9.4.

Proposition 9.2. For $0<p<p_{c}$, and for all dimensions $d \geq 2$,

$$
\frac{\mathrm{d} \chi(p)}{\mathrm{d} p} \leq|\Omega| \chi(p)^{2} .
$$

In addition, $\lim _{p \rightarrow p_{c}^{-}} \chi(p)=\infty$.

The following corollary to the proposition can be interpreted as the meanfield bound $\gamma \geq 1$, valid in all dimensions $d \geq 2$.

Corollary 9.3. For $0<p<p_{c}$, and for all dimensions $d \geq 2$,

$$
\chi(p) \geq \frac{1}{|\Omega|\left(p_{c}-p\right)} .
$$

Proof. The inequality (9.22) can be rewritten as

$$
-\frac{\mathrm{d} \chi(p)^{-1}}{\mathrm{~d} p} \leq|\Omega| .
$$

Integration over the interval $\left[p, p_{2}\right]$ then gives

$$
\chi(p)^{-1}-\chi\left(p_{2}\right)^{-1} \leq|\Omega|\left(p_{2}-p\right) .
$$

Taking the limit $p_{2} \rightarrow p_{c}^{-}$and applying the last statement in the proposition proves the corollary.

Partial differential inequalities for the magnetization are given in the following proposition, which is due to [7].

Proposition 9.4. For $0<p<1$ and $0<\gamma<1$, and for all dimensions $d \geq 2$,

$$
\begin{gathered}
(1-p) \frac{\partial M}{\partial p} \leq|\Omega|(1-\gamma) M \frac{\partial M}{\partial \gamma}, \\
M \leq \gamma \frac{\partial M}{\partial \gamma}+M^{2}+p M \frac{\partial M}{\partial p} .
\end{gathered}
$$

The derivative of $M$ with respect to $p$ can be eliminated from the above pair of inequalities, by inserting (9.26) into (9.27) to obtain

$$
M \leq \gamma \frac{\partial M}{\partial \gamma}+M^{2}+\frac{p|\Omega|}{1-p}(1-\gamma) M^{2} \frac{\partial M}{\partial \gamma} .
$$


We omit the proof of Proposition 9.4, which can be found in [7] or [85, Section 5.3]. Instead, we will show how (9.26)-(9.27) can be integrated to prove the following theorem from [7]. For its statement, we define

$$
\begin{aligned}
\chi(p, \gamma) & =(1-\gamma) \frac{\partial M(p, \gamma)}{\partial \gamma} \\
& =\sum_{n=1}^{\infty} n(1-\gamma)^{n} P_{n}(p)
\end{aligned}
$$

and

$$
\begin{aligned}
\chi^{f}(p) & =\mathbb{E}_{p}[|C(0)| I[|C(0)|<\infty]] \\
& =\sum_{n=1}^{\infty} n P_{n}(p)=\chi(p, 0)
\end{aligned}
$$

(the superscript $f$ stands for finite).

Theorem 9.5. Fix $p$ with $\chi^{f}(p)=\infty$. There is a positive constant $a=a(p)$ such that

$$
M(p, \gamma) \geq a \gamma^{1 / 2}
$$

In addition, either $\theta(p)>0$, or it is the case that $\theta(p)=0$ and $\theta\left(p^{\prime}\right) \geq$ $\frac{1}{2 p^{\prime}}\left(p^{\prime}-p\right)$ for all $p^{\prime} \geq p$.

Exercise 9.6. Define $p_{c}=\inf \{p: \theta(p)>0\}$ and $\pi_{c}=\sup \{p: \chi(p)<\infty\}$.

(a) Prove that $\pi_{c} \leq p_{c}$.

(b) Prove that $\pi_{c}=p_{c}$. Hint: assume that $\pi_{c}<p_{c}$ and derive a contradiction from Theorem 9.5.

The bounds of Theorem 9.5 can be interpreted as the mean-field bounds $\delta \geq 2$ and $\beta \leq 1$. In fact, if $M\left(p_{c}, 0\right)=\theta\left(p_{c}\right)>0$ then certainly $M\left(p_{c}, \gamma\right) \geq$ $\theta\left(p_{c}\right) \geq \theta\left(p_{c}\right) \gamma^{1 / 2}$. On the other hand, if $\theta\left(p_{c}\right)=0$ then $\chi^{f}\left(p_{c}\right)=\chi\left(p_{c}\right)=\infty$ (by Corollary 9.3), and hence

$$
M\left(p_{c}, \gamma\right) \geq a \gamma^{1 / 2}
$$

by Theorem 9.5 . This says that $\delta \geq 2$. Similarly, if $\theta\left(p_{c}\right)=0$ then Theorem 9.5 implies that

$$
\theta(p) \geq \frac{1}{2 p}\left(p-p_{c}\right)
$$

for $p \geq p_{c}$, so that $\beta \leq 1$. On the other hand, if $\theta\left(p_{c}\right)>0$ then we similarly have $\theta(p) \geq \theta\left(p_{c}\right) \geq \theta\left(p_{c}\right)\left(p-p_{c}\right)$, for $p \geq p_{c}$.

Proof of Theorem 9.5. We fix $p$ with $\chi^{f}(p)=\infty$, and drop the $p$ dependence from the notation. We first prove (9.31). Note that (9.31) is vacuous if $M(p, 0)>0$, so we may assume that $M(p, 0)=0$. 
In (9.28), we write $A=\frac{p|\Omega|}{1-p}$, and use $1-\gamma \leq 1$, to obtain

$$
M \leq \gamma \frac{\mathrm{d} M}{\mathrm{~d} \gamma}+M^{2}+A M^{2} \frac{\mathrm{d} M}{\mathrm{~d} \gamma}
$$

We have used ordinary, rather than partial derivatives, in (9.34), since we are considering $p$ to be fixed. We use the fact that $M>0$ when $\gamma>0$, and that $M$ has a well-defined inverse function $\gamma=\gamma(M)$. Multiplication of (9.34) by $\frac{1}{M} \frac{\mathrm{d} \gamma}{\mathrm{d} M}$ gives

$$
\frac{\mathrm{d} \gamma}{\mathrm{d} M} \leq \frac{\gamma}{M}+M \frac{\mathrm{d} \gamma}{\mathrm{d} M}+A M
$$

Equivalently,

$$
\frac{\mathrm{d} \gamma}{\mathrm{d} M}-\frac{\gamma}{M(1-M)} \leq A \frac{M}{1-M}
$$

We multiply (9.36) by the integrating factor $\exp \left[-\int \frac{1}{M(1-M)} \mathrm{d} M\right]=\frac{1-M}{M}$ to obtain

$$
\frac{\mathrm{d}}{\mathrm{d} M}\left[\frac{\gamma(1-M)}{M}\right] \leq A .
$$

Integration over the interval $[0, M]$ then gives

$$
\frac{\gamma(1-M)}{M}-\lim _{M \rightarrow 0} \frac{\gamma(1-M)}{M} \leq A M
$$

To evaluate the limit, we observe that $\gamma(0)=0$ (recall that we are assuming $M(p, 0)=0)$, and conclude from the mean-value theorem and (9.29) that there is a $\gamma^{*} \in(0, \gamma)$ such that

$$
\frac{M}{\gamma}=\left.\frac{\mathrm{d} M}{\mathrm{~d} \gamma}\right|_{\gamma=\gamma^{*}}=\frac{1}{1-\gamma^{*}} \chi\left(p, \gamma^{*}\right) \rightarrow \chi^{f}(p)=\infty
$$

as $\gamma \rightarrow 0$. Therefore,

$$
\frac{\gamma(1-M)}{M} \leq A M
$$

Since $M \leq \frac{1}{2}$ for small $\gamma$ (because $M \rightarrow 0$ as $\gamma \rightarrow 0$ ), it follows that

$$
M^{2} \geq \frac{1}{2 A} \gamma
$$

for small $\gamma$, which proves $(9.31)$.

Next, we consider the statement for $\theta$. If $\theta(p)>0$ then we are done, so we assume $\theta(p)=0$. We multiply (9.27) by $\frac{1}{\gamma M}$ to obtain

$$
0 \leq \frac{\partial \log M}{\partial \gamma}+\frac{1}{\gamma} \frac{\partial}{\partial \tilde{p}}(\tilde{p} M-\tilde{p}),
$$


where $\tilde{p} \in(0,1)$ is arbitrary (and $M$ is evaluated at $\tilde{p}$ ). We fix $0<\gamma_{1}<\gamma_{2}<1$ and integrate (9.42) with respect to $\gamma$ over the interval $\left[\gamma_{1}, \gamma_{2}\right]$, and with respect to $\tilde{p}$ over the interval $\left[p, p^{\prime}\right]$.

For the first term, we integrate first with respect to $\gamma$, and use the monotonicity of $M$, to obtain

$$
\log M\left(\tilde{p}, \gamma_{2}\right)-\log M\left(\tilde{p}, \gamma_{1}\right) \leq \log M\left(p^{\prime}, \gamma_{2}\right)-\log M\left(p, \gamma_{1}\right),
$$

obtaining

$$
\left(p^{\prime}-p\right)\left[\log M\left(p^{\prime}, \gamma_{2}\right)-\log M\left(p, \gamma_{1}\right)\right]
$$

as an upper bound for the double integral of the first term.

For the second term, we integrate first with respect to $\tilde{p}$, using

$$
p^{\prime} M\left(p^{\prime}, \gamma\right)-p M(p, \gamma)-\left(p^{\prime}-p\right) \leq p^{\prime} M\left(p^{\prime}, \gamma_{2}\right)-p^{\prime}+p,
$$

and then complete the integration to obtain the upper bound

$$
\left[p^{\prime} M\left(p^{\prime}, \gamma_{2}\right)-p^{\prime}+p\right] \log \frac{\gamma_{2}}{\gamma_{1}}
$$

for the double integral of the second term.

Altogether, this gives

$$
0 \leq\left(p^{\prime}-p\right)\left[\frac{\log M\left(p^{\prime}, \gamma_{2}\right)}{\log \frac{\gamma_{2}}{\gamma_{1}}}-\frac{\log M\left(p, \gamma_{1}\right)}{\log \frac{\gamma_{2}}{\gamma_{1}}}\right]+p^{\prime} M\left(p^{\prime}, \gamma_{2}\right)-p^{\prime}+p .
$$

Now we let $\gamma_{1} \rightarrow 0$. The first term vanishes in this limit. For the second term, we use (9.31) to conclude that

$$
-\frac{\log M\left(p, \gamma_{1}\right)}{\log \frac{\gamma_{2}}{\gamma_{1}}} \leq \frac{-\log a-\frac{1}{2} \log \gamma_{1}}{\log \gamma_{2}-\log \gamma_{1}} .
$$

Since the right hand side approaches $\frac{1}{2}$ as $\gamma_{1} \rightarrow 0$, we obtain

$$
0 \leq \frac{1}{2}\left(p^{\prime}-p\right)+p^{\prime} M\left(p^{\prime}, \gamma_{2}\right)-p^{\prime}+p,
$$

which implies that

$$
M\left(p^{\prime}, \gamma_{2}\right) \geq \frac{1}{2 p^{\prime}}\left(p^{\prime}-p\right) .
$$

Now we take the limit $\gamma_{2} \rightarrow 0^{-}$to complete the proof.

\subsection{Differential Inequalities and the Triangle Condition}

In this section, we state differential inequalities involving the triangle diagram which are complementary to the differential inequalities (9.22) for $\chi$ and (9.28) for $M$. We also derive consequences of these differential inequalities. 
Let

$$
\nabla_{p}(w)=\sum_{x, y \in \mathbb{Z}^{d}} \tau_{p}(x) \tau_{p}(y-x) \tau_{p}(w-y) .
$$

By Exercise 1.1,

$$
\hat{\nabla}_{p}(k)=\hat{\tau}_{p}(k)^{3} .
$$

The triangle diagram is defined by

$$
\mathrm{T}(p)=\nabla_{p}(0)=\int_{[-\pi, \pi]^{d}} \hat{\tau}_{p}(k)^{3} \frac{\mathrm{d}^{d} k}{(2 \pi)^{d}},
$$

and the triangle condition is the statement that $\mathrm{T}\left(p_{c}\right)<\infty$. We also define

$$
\overline{\mathbf{T}}(p)=\sup _{w \in \mathbb{Z}^{d}}\left[\nabla_{p}(w)-\delta_{w, 0}\right] .
$$

A proof that $\hat{\tau}_{p}(k) \geq 0$ is given in [12], so the integrand of (9.53) is nonnegative. If we insert the conjectured behaviour $\hat{\tau}_{p_{c}}(k) \sim c|k|^{\eta-2}$ into the integral of (9.53), the result is finite if $d>6-3 \eta$, and infinite otherwise. The mean-field value of $\eta$ is zero, $\eta=\frac{5}{24}$ for $d=2$ (this has currently been proven only for site percolation on the triangular lattice [191]), and $\eta$ is conjectured to be negative for $2<d<6$ [1]. Thus, the triangle condition should hold if and only if $d>6$.

The following differential inequality is complementary to (9.22). We defer its proof to Section 9.4.

Proposition 9.7. For $0<p<p_{c}$, and for all dimensions $d \geq 2$,

$$
\frac{\mathrm{d} \chi(p)}{\mathrm{d} p} \geq[1-\overline{\mathbf{T}}(p)]|\Omega| \chi(p)^{2} .
$$

The following corollary to the proposition shows that $\gamma \leq 1$ if $\overline{\mathbf{T}}\left(p_{c}\right)<$ 1. Since we have seen quite generally that $\gamma \geq 1$, this means that $\gamma=1$ if $\overline{\mathbf{T}}\left(p_{c}\right)<1$. In fact, it can be shown that $\gamma \leq 1$ whenever the triangle condition $\mathbf{T}\left(p_{c}\right)<\infty$ holds [12], rather than the stronger assumption $\overline{\mathbf{T}}\left(p_{c}\right)<$ 1. However, in practice, the stronger conclusion $\overline{\mathbf{T}}\left(p_{c}\right)<1$ is what has been proved using the lace expansion, and this will suffice for our purposes. (As in the discussion of Section 5.3, it would be desirable to find an approach to the lace expansion that is based on the finiteness of the triangle diagram, rather than on its smallness.)

Corollary 9.8. Let $d \geq 2$ and $0 \leq p<p_{c}$. If $\overline{\mathbf{T}}\left(p_{c}\right)<1$ then

$$
\chi(p) \leq \frac{1}{\left[1-\overline{\mathbf{T}}\left(p_{c}\right)\right]|\Omega|\left(p_{c}-p\right)} .
$$


Proof. By monotonicity, $\overline{\mathbf{T}}(p)$ can be replaced by $\overline{\mathbf{T}}\left(p_{c}\right)$ in (9.55). As in the proof of Corollary 9.3, integration of the resulting inequality over $\left(p_{1}, p_{2}\right)$ (with $\left.p_{2}<p_{c}\right)$ gives

$$
\chi\left(p_{1}\right)^{-1}-\chi\left(p_{2}\right)^{-1} \geq\left[1-\overline{\mathbf{T}}\left(p_{c}\right)\right]|\Omega|\left(p_{2}-p_{1}\right) .
$$

Taking $p_{1}=p$ and $p_{2} \rightarrow p_{c}^{-}$, the desired result then follows from the fact that $\chi(p) \rightarrow \infty$ as $p \rightarrow p_{c}^{-}$, by Proposition 9.2.

For the magnetization, we have the following differential inequality involving the triangle. This differential inequality is a variant of an inequality derived by [20], and is complementary to (9.28). It was proved in essentially this form in [31]. The proof will be given in Section 9.4. A factor $M$ can be cancelled on each side of (9.58), but we state the inequality in this form because this is how it is proved, and for comparison with (9.28).

Proposition 9.9. Let $0<\gamma<1$ and $p \in\left[|\Omega|^{-1}, p_{c}\right)$. There is a universal constant $a_{0}$ such that if $\overline{\mathrm{T}}\left(p_{c}\right)$ is sufficiently small then

$$
M \geq a_{0} p|\Omega|(1-\gamma) M^{2} \frac{\partial M}{\partial \gamma} .
$$

The following theorem is a consequence of Proposition 9.9. When combined with Theorem 9.5, it shows that $M\left(p_{c}, \gamma\right) \simeq \gamma^{1 / 2}$ and $\theta(p) \simeq\left(p_{c}-p\right)$, i.e., that the critical exponents $\delta$ and $\beta$ exist and take their mean-field values $\delta=2$ and $\beta=1$, whenever $\overline{\mathbf{T}}\left(p_{c}\right)$ is sufficiently small. This will be sufficient for our needs, but it should be noted that in [20] the same conclusion is derived under the weaker assumption that $\mathrm{T}\left(p_{c}\right)$ is finite. Our proof of Theorem 9.10 is based on [31].

Theorem 9.10. Let $a_{1}^{2}=\max \left\{2,4 / a_{0}\right\}$, where $a_{0}$ is the constant of Proposition 9.9. If $\overline{\mathbf{T}}\left(p_{c}\right)$ is sufficiently small, then for $\gamma \geq 0$ and $p \geq p_{c}$,

$$
\begin{aligned}
M\left(p_{c}, \gamma\right) & \leq a_{1} \gamma^{1 / 2}, \\
\theta(p) & \leq 4 a_{1}^{2}|\Omega|\left(p-p_{c}\right) .
\end{aligned}
$$

In particular, $\theta\left(p_{c}\right)=0$.

Proof. We begin with the proof of the upper bound on $M\left(p_{c}, \gamma\right)$. Suppose first that $\gamma \leq \frac{1}{2}$, so that $1-\gamma \geq \frac{1}{2}$. Let $p \in\left[|\Omega|^{-1}, p_{c}\right.$ ) (it is a standard fact that $p_{c}>|\Omega|^{-1}$; see $\left.[85,(1.13)]\right)$. Then $p|\Omega| \geq 1$ and it follows from (9.58) that

$$
\frac{\partial M^{2}}{\partial \gamma} \leq \frac{4}{a_{0}} \leq a_{1}^{2}
$$

Therefore,

$$
M(p, \gamma)^{2}-M(p, 0)^{2} \leq a_{1}^{2} \gamma
$$


Since $p<p_{c}$, the subtracted term on the right hand side is zero. Taking the limit $p \rightarrow p_{c}^{-}$, and using the continuity of $M(p, \gamma)$ in $p$ for $\gamma>0$, we obtain the desired bound

$$
M\left(p_{c}, \gamma\right) \leq a_{1} \gamma^{1 / 2}
$$

for $\gamma>0$. The same bound then holds for $\gamma=0$ due to the monotonicity of $M\left(p_{c}, \gamma\right)$ in $\gamma$. On the other hand, if $\gamma \geq \frac{1}{2}$, then

$$
M\left(p_{c}, \gamma\right)^{2} \leq 1 \leq 2 \gamma,
$$

which gives (9.59) in this case.

To extend (9.59) to (9.60), we apply an extrapolation principle [20, 7, 9, 73]. The extrapolation principle converts the upper bound $(9.59)$ on $M\left(p_{c}, \gamma\right)$ to an upper bound valid for $p>p_{c}$. This is perhaps surprising, since $M$ is an increasing function of $p$. However, it is also increasing in $\gamma$, and the differential inequality (9.26) can be used to compensate for an increase in $p$ with a decrease in $\gamma$.

In our context of nearest-neighbour or spread-out bond percolation on $\mathbb{Z}^{d}$, we have $p_{c} \leq \frac{1}{2}$. Suppose first that $p>\frac{3}{4}$. Then

$$
\theta(p) \leq 1 \leq 4\left(p-p_{c}\right) \leq 4 a_{1}^{2}|\Omega|\left(p-p_{c}\right) .
$$

Thus we may assume that $p \in\left(p_{c}, \frac{3}{4}\right)$, and we make this assumption throughout the rest of the proof.

We find it convenient to make the change of variables $\gamma=1-\mathrm{e}^{-h}$, and define $\tilde{M}(p, h)=M\left(p, 1-\mathrm{e}^{-h}\right)$, for $h \geq 0$. Since $p \leq \frac{3}{4}$, the differential inequality (9.26) implies that

$$
\frac{\partial \tilde{M}}{\partial p} \leq 4|\Omega| \tilde{M} \frac{\partial \tilde{M}}{\partial h} .
$$

For fixed fixed $p \in(0,1)$ and $m \in[\theta(p), 1)$, we can solve the equation $\tilde{M}(p, h)=m$ for $h=h(p)$, so that $\tilde{M}(p, h(p))=m$. Differentiation of this identity with respect to $p$ gives

$$
\frac{\partial \tilde{M}}{\partial p}+\left.\frac{\partial \tilde{M}}{\partial h} \frac{\partial h}{\partial p}\right|_{\tilde{M}=m}=0 .
$$

Therefore,

$$
0 \leq-\left.\frac{\partial h}{\partial p}\right|_{\tilde{M}=m}=\frac{\frac{\partial \tilde{M}}{\partial p}}{\frac{\partial \tilde{M}}{\partial h}} \leq 4|\Omega| m .
$$

Choose $p \in\left(p_{c}, \frac{3}{4}\right)$, let $P_{1}$ be the point $(p, 0)$, and set $m_{1}=\tilde{M}(p, 0)$. The bound $-\left.\frac{\partial h}{\partial p}\right|_{\tilde{M}=m_{1}} \leq 4|\Omega| m_{1}$ implies that the point $P_{2}=\left(p_{c}, 4|\Omega| m_{1}\left(p-p_{c}\right)\right)$ lies above the contour line $\tilde{M}=m_{1}$ in the $(p, h)$ plane (see Fig. 9.2). Since 


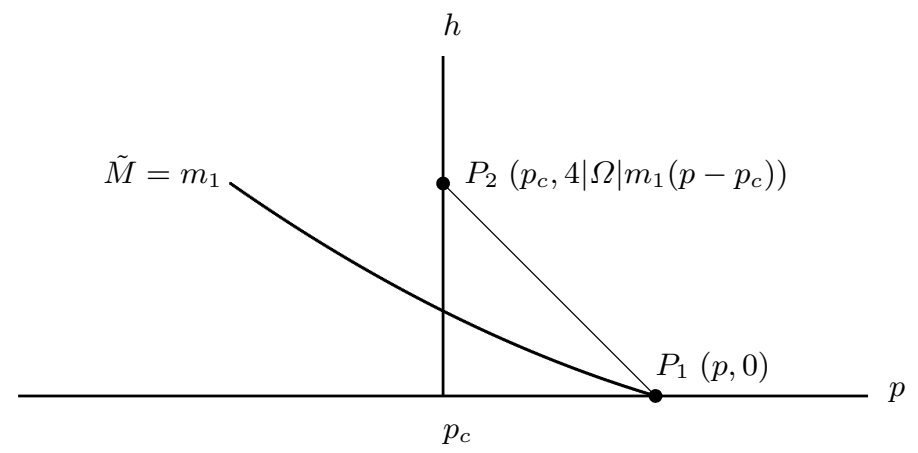

Fig. 9.2. The extrapolation geometry.

$\tilde{M}\left(p_{c}, h\right)$ is monotone in $h$, the value of $\tilde{M}$ at $P_{2}$ is bounded below by the value at $P_{1}$.

Therefore,

$$
\theta(p)=M(p, 0) \leq M\left(p_{c}, 4|\Omega| \theta(p)\left(p-p_{c}\right)\right) .
$$

Applying (9.59) gives

$$
\theta(p) \leq a_{1} \sqrt{4|\Omega| \theta(p)\left(p-p_{c}\right)},
$$

which implies that

$$
\theta(p) \leq 4 a_{1}^{2}|\Omega|\left(p-p_{c}\right)
$$

This completes the proof.

\subsection{Proofs of the Differential Inequalities}

\subsubsection{Differential Inequality for the Susceptibility}

In this section, we sketch the proof of the differential inequalities for the susceptibility given in Propositions 9.2 and 9.7, which are restated here as Proposition 9.11. We follow the original reasoning of Aizenman and Newman [12].

Proposition 9.11. For $d \geq 2$ and $0<p<p_{c}$,

$$
[1-\overline{\mathbf{T}}(p)]|\Omega| \chi(p)^{2} \leq \frac{\mathrm{d} \chi(p)}{\mathrm{d} p} \leq|\Omega| \chi(p)^{2} .
$$

In addition, $\lim _{p \rightarrow p_{c}^{-}} \chi(p)=\infty$. 
A complete proof requires an approximation of $\mathbb{Z}^{d}$ by a finite graph, followed by a limiting argument. This is quite similar to the argument used in the proof of Theorem 7.6, and we will omit the details here. Full details can be found in [12]. The proof that $\chi$ diverges as $p$ approaches $p_{c}^{-}$uses the finite volume argument, and will also be omitted here.

We write $\mathbb{B}$ for the set of all bonds. Given a bond configuration, a bond $\{u, v\} \in \mathbb{B}$ (occupied or not) is called pivotal for the connection from $x$ to $y$ if $x \leftrightarrow y$ in the possibly modified configuration in which the bond is made occupied, whereas $x$ is not connected to $y$ in the possibly modified configuration in which the bond is made vacant. Bonds are not usually regarded as directed. However, it will be convenient at times to regard a bond $\{u, v\}$ as directed from $u$ to $v$, and we will emphasize this point of view with the notation $(u, v)$. A directed bond $(u, v)$ is pivotal for the connection from $x$ to $y$ if $x \leftrightarrow u$, if $v \leftrightarrow y$, and if $x$ is not connected to $y$ if $\{u, v\}$ is made vacant.

Let $E \circ F$ denote the event that the events $E$ and $F$ occur disjointly. In our applications, $E$ and $F$ will be events that certain connections take place, and in this case $E \circ F$ is the event that both $E$ and $F$ occur and that given a configuration it is possible to find one set of bonds providing the connections needed by $E$ and a disjoint set of bonds providing the connections needed by $F$. The BK inequality implies that for such events $E$ and $F, \mathbb{P}_{p}(E \circ$ $F) \leq \mathbb{P}_{p}(E) \mathbb{P}_{p}(F)$ (see [85, Theorem 2.12] for a more complete and precise discussion). The BK inequality has great importance in percolation theory, and will be an key ingredient in our analysis. We will use it in the form of the following proposition. The proposition plays the role for percolation played by subadditive estimates for the self-avoiding walk and lattice trees, and expresses a repulsive character of the model.

Proposition 9.12. Let $V_{1}, V_{2}, \ldots, V_{n}$ be sets of paths in the lattice, and let $E_{i}(i=1, \ldots, n)$ be the event that at least one of the paths in $V_{i}$ is occupied. Let $F$ be the event that there are pairwise edge-disjoint occupied paths from each of the sets $V_{1}, V_{2}, \ldots, V_{n}$. Then

$$
\mathbb{P}_{p}(F) \leq \prod_{i=1}^{n} \mathbb{P}_{p}\left(E_{i}\right) .
$$

Sketch of proof of the upper bound in (9.72). By Russo's formula (see [85, Theorem 2.25]),

$$
\begin{aligned}
\frac{\mathrm{d}}{\mathrm{d} p} \tau_{p}(x, y) & =\sum_{\{u, v\} \in \mathbb{B}} \mathbb{P}_{p}(\{u, v\} \text { is pivotal for } x \leftrightarrow y) \\
& =\sum_{(u, v)} \mathbb{P}_{p}((u, v) \text { is pivotal for } x \leftrightarrow y),
\end{aligned}
$$

where the sum over $(u, v)$ is a sum over directed bonds. The above is fine for a finite graph, but is not directly legitimate for an infinite graph. We will ignore this subtlety. By (9.73) and the BK inequality, 


$$
\frac{\mathrm{d}}{\mathrm{d} p} \tau_{p}(x, y) \leq \sum_{(u, v)} \mathbb{P}_{p}(\{x \leftrightarrow u\} \circ\{v \leftrightarrow y\}) \leq \sum_{(u, v)} \tau_{p}(x, u) \tau_{p}(v, y) .
$$

We then perform the sums over $y, v, u$ (in that order) and use translation invariance to obtain the desired upper bound.

For the lower bound of (9.72), we will use the following definition.

Definition 9.13. (a) Given a bond configuration, and a random or deterministic set $A \subset \mathbb{Z}^{d}$, we say $x$ and $y$ are connected in $A$ if there is an occupied path from $x$ to $y$ whose vertices are all in $A$, or if $x=y \in A$. We define $a$ restricted two-point function by

$$
\tau^{A}(x, y)=\mathbb{P}_{p}\left(x \leftrightarrow y \text { in } \mathbb{Z}^{d} \backslash A\right) .
$$

(b) Given a bond configuration, and a random or deterministic set $A \subset \mathbb{Z}^{d}$, we say $x$ and $y$ are connected through $A$, denoted $x \stackrel{A}{\leftrightarrow} y$, if $x=y \in A$ or if $x \leftrightarrow y$ and every occupied path connecting $x$ to $y$ has at least one bond with an endpoint in $A$, i.e., if $x$ is connected to $y$ but it is not the case that $x \leftrightarrow y$ in $\mathbb{Z}^{d} \backslash A$.

(c) Given a bond configuration, and a bond b, we define $\tilde{C}^{b}(x)$ to be the set of vertices connected to $x$ in the new configuration obtained by setting $b$ to be vacant.

Exercise 9.14. Prove that

$$
\begin{aligned}
& \{(u, v) \text { pivotal for } 0 \leftrightarrow x\} \\
& \quad=\left\{0 \leftrightarrow u \text { in } \tilde{C}^{(u, v)}(0)\right\} \cap\left\{v \leftrightarrow x \text { in } \mathbb{Z}^{d} \backslash \tilde{C}^{(u, v)}(0)\right\} .
\end{aligned}
$$

Sketch of proof of the lower bound in (9.72). Conditioning on $\tilde{C}^{(u, v)}(0)$, it follows from (9.76) that

$$
\begin{aligned}
& \mathbb{P}_{p}((u, v) \text { pivotal for } 0 \leftrightarrow x) \\
& \quad=\sum_{A: A \ni 0} \mathbb{P}_{p}\left(\tilde{C}^{(u, v)}(0)=A, 0 \leftrightarrow u \text { in } A, v \leftrightarrow x \text { in } \mathbb{Z}^{d} \backslash A\right) \\
& =\sum_{A: A \ni 0} \mathbb{P}_{p}\left(\tilde{C}^{(u, v)}(0)=A, 0 \leftrightarrow u \text { in } A\right) \mathbb{P}_{p}\left(v \leftrightarrow x \text { in } \mathbb{Z}^{d} \backslash A\right) \\
& =\sum_{A: A \ni 0} \mathbb{P}_{p}\left(\tilde{C}^{(u, v)}(0)=A, 0 \leftrightarrow u\right) \tau_{p}^{A}(v, x) .
\end{aligned}
$$

Here, the sum over $A$ is the sum over lattice animals containing 0 . Also, we have used the fact that the events within the probabilities in the third line are independent, and, in the last line, that if $0 \leftrightarrow u$ but it is not the case that $0 \leftrightarrow u$ in $A$ then $v$ must be in $A$ and hence $\tau_{p}^{A}(v, x)=0$. It follows that

$$
\mathbb{P}_{p}((u, v) \text { pivotal for } 0 \leftrightarrow x)=\mathbb{E}_{p}\left(I[0 \leftrightarrow u] \tau^{\tilde{C}^{(u, v)}(0)}(v, x)\right) .
$$


In the nested expectation on the right hand side of (9.78), the set $\tilde{C}^{(u, v)}(w)$ is a random set with respect to the expectation $\mathbb{E}_{p}$, but it is deterministic with respect to the expectation defining the restricted two-point function. The latter expectation effectively introduces a second percolation model on a second lattice, which is coupled to the original percolation model via the set $\tilde{C}^{(u, v)}(0)$.

Since

$$
\begin{aligned}
\tau_{p}^{A}(v, x) & =\tau_{p}(v, x)-\left[\tau_{p}(v, x)-\tau_{p}^{A}(v, x)\right] \\
& =\tau_{p}(v, x)-\mathbb{P}_{p}(v \stackrel{A}{\leftrightarrow} x),
\end{aligned}
$$

the identity (9.78) can be rewritten as

$$
\begin{aligned}
& \mathbb{P}_{p}((u, v) \text { pivotal for } 0 \leftrightarrow x) \\
& \quad=\tau_{p}(0, u) \tau_{p}(v, x)-\mathbb{E}_{p}\left(I[0 \leftrightarrow u] \mathbb{P}_{p}\left(v \stackrel{\tilde{C}^{(u, v)}(0)}{\longrightarrow} x\right)\right) .
\end{aligned}
$$

By the BK inequality, for $A \subset \mathbb{Z}^{d}$ we have

$$
\begin{aligned}
\mathbb{P}_{p}(v \stackrel{A}{\longleftrightarrow} x) & \leq \mathbb{P}_{p}\left(\bigcup_{y \in A}\{v \leftrightarrow y\} \circ\{y \leftrightarrow x\}\right) \\
& \leq \sum_{y \in \mathbb{Z}^{d}} I[y \in A] \mathbb{P}_{p}(\{v \leftrightarrow y\} \circ\{y \leftrightarrow x\}) \\
& \leq \sum_{y \in \mathbb{Z}^{d}} I[y \in A] \tau_{p}(v, y) \tau_{p}(y, x) .
\end{aligned}
$$

Therefore, for $A=\tilde{C}^{(u, v)}(0) \subset C(0)$, we have

$$
\mathbb{P}_{p}\left(v \stackrel{\tilde{C}^{(u, v)}(0)}{\longrightarrow} x\right) \leq \sum_{y \in \mathbb{Z}^{d}} I[y \in C(0)] \tau_{p}(v, y) \tau_{p}(y, x) .
$$

Substitution yields

$$
\begin{aligned}
& \mathbb{P}_{p}((u, v) \text { pivotal for } 0 \leftrightarrow x) \\
& \quad \geq \tau_{p}(0, u) \tau_{p}(v, x)-\sum_{y \in \mathbb{Z}^{d}} \mathbb{P}_{p}(0 \leftrightarrow u, 0 \leftrightarrow y) \tau_{p}(v, y) \tau_{p}(y, x) .
\end{aligned}
$$

The tree-graph bound [12], which is an elementary consequence of the BK inequality, implies that

$$
\mathbb{P}_{p}(0 \leftrightarrow u, 0 \leftrightarrow y) \leq \sum_{z \in \mathbb{Z}^{d}} \tau_{p}(0, z) \tau_{p}(z, y) \tau_{p}(z, u) .
$$

Therefore, 
$\mathbb{P}_{p}((u, v)$ pivotal for $0 \leftrightarrow x) \geq \tau_{p}(0, u) \tau_{p}(v, x)$

$$
-\sum_{y, z \in \mathbb{Z}^{d}} \tau_{p}(0, z) \tau_{p}(z, y) \tau_{p}(z, u) \tau_{p}(y, v) \tau_{p}(y, x) .
$$

See Fig. 9.3. Recalling (9.73) (again we are careless about the infinite volume limit), and performing the sums over $u, v, x$, leads to

$$
\begin{aligned}
\frac{\mathrm{d} \chi(p)}{\mathrm{d} p} & \geq|\Omega| \chi(p)^{2}-\chi(p) \sum_{z \in \mathbb{Z}^{d}} \tau_{p}(0, z) \sum_{(u, v)} \sum_{y \in \mathbb{Z}^{d}} \tau_{p}(z, y) \tau_{p}(z, u) \tau_{p}(y, v) \\
& =|\Omega| \chi(p)^{2}-\chi(p) \sum_{z \in \mathbb{Z}^{d}} \tau_{p}(0, z)|\Omega| \sup _{e \in \Omega} \nabla_{p}(e) \\
& \geq|\Omega| \chi(p)^{2}[1-\overline{\mathbf{T}}(p)],
\end{aligned}
$$

using symmetry in the second step.

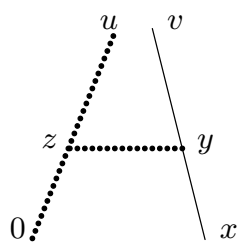

Fig. 9.3. The subtracted term in (9.85). The dotted lines arise from paths in $\tilde{C}^{\{u, v\}}(0)$, while the solid line arises from a connection from $v$ to $x$ through $\tilde{C}^{\{u, v\}}(0)$.

\subsubsection{Differential Inequality for the Magnetization}

In this section, we prove the differential inequality (9.58), following the method of [31]. Our proof is related to, but simpler than, the method used in [20] to prove an inequality useful under the weaker assumption that the triangle condition holds (rather than that $\overline{\mathbf{T}}\left(p_{c}\right)$ is small). Nevertheless, the proof is not simple. Results related to (9.58) can also be found in [104, Section 3].

We restate (9.58) as (9.87) in the following proposition. Note that, by (9.29), the factor $(1-\gamma) \partial M / \partial \gamma$ on the right hand side of (9.58) can be replaced by $\chi(p, \gamma)$.

Proposition 9.15. Let $0<\gamma<1$ and $p \in\left[|\Omega|^{-1}, p_{c}\right)$. There is a universal constant $a_{0}$ such that if $\overline{\mathbf{T}}\left(p_{c}\right)$ is sufficiently small then

$$
M \geq a_{0} p|\Omega| M^{2} \chi(p, \gamma) .
$$

Before beginning the proof, we discuss some preliminaries. Recall from $(9.10)$ that $M(p, \gamma)=\mathbb{P}_{p, \gamma}(0 \leftrightarrow \mathcal{G})$. We define $\{x \leftrightarrow \mathcal{G}\}$ to be the event that 
$C(x) \cap \mathcal{G} \neq \varnothing$. We say that $(u, v)$ is pivotal for $x \leftrightarrow \mathcal{G}$ if $x \leftrightarrow u$, if $v \leftrightarrow \mathcal{G}$, and if $x \leftrightarrow \mathcal{G}$ when $(u, v)$ is made vacant. Let $\{v \Leftrightarrow \mathcal{G}\}$ denote the event that there exist $x, y \in \mathcal{G}$, with $x \neq y$, such that there are disjoint connections $v \leftrightarrow x$ and $v \leftrightarrow y$. Let $F_{(u, v)}$ denote the event that (i) the bond $(u, v)$ is occupied and pivotal for the connection from 0 to $\mathcal{G}$, and that (ii) $\{v \Leftrightarrow \mathcal{G}\}$. Let $F=\cup_{(u, v)} F_{(u, v)}$, and note that the union is disjoint. Since $0 \leftrightarrow \mathcal{G}$ when $F$ occurs,

$$
M=\mathbb{P}_{p, \gamma}(0 \leftrightarrow \mathcal{G}) \geq \mathbb{P}_{p, \gamma}(F)=\sum_{(u, v)} \mathbb{P}_{p, \gamma}\left(F_{(u, v)}\right),
$$

and it suffices to prove that $\mathbb{P}_{p, \gamma}(F)$ is bounded below by the right hand side of (9.87).

Let $0<\gamma<1$. For $x, y \in \mathbb{V}$, we define a "green-free" analogue of the two-point function by

$$
\tau_{p, \gamma}(x, y)=\mathbb{P}_{p, \gamma}(x \leftrightarrow y, x \nLeftarrow \mathcal{G}),
$$

so that

$$
\chi(p, \gamma)=\sum_{x \in \mathbb{Z}^{d}} \tau_{p, \gamma}(0, x) .
$$

Exercise 9.16. Prove (9.90). (Recall (9.29).)

Given a subset $A \subset \mathbb{V}$, we define $\tau_{p, \gamma}^{A}(x, y)$ to be the probability that (i) $x \leftrightarrow y$ in $\mathbb{Z}^{d} \backslash A$, and (ii) $x \nLeftarrow \mathcal{G}$ in $\mathbb{Z}^{d} \backslash A$, which is to say that $x \nLeftarrow \mathcal{G}$ after every bond with an endpoint in $A$ is made vacant. We write $\tilde{I}^{\{u, v\}}[E]$ for the indicator that $E$ occurs after $\{u, v\}$ is made vacant.

\section{Lemma 9.17.}

$$
\mathbb{P}_{p, \gamma}\left(F_{(u, v)}\right)=p \mathbb{E}_{p, \gamma}\left[\tau_{p, \gamma}^{\tilde{C}^{(u, v)}(v)}(0, u) \tilde{I}^{\{u, v\}}[v \Leftrightarrow \mathcal{G}]\right] .
$$

Proof. We first observe that the event $F_{(u, v)}$ is equal to

$$
\begin{aligned}
&\left\{0 \leftrightarrow u \text { in } \mathbb{Z}^{d} \backslash \tilde{C}^{(u, v)}(v)\right\} \cap\left\{0 \leftrightarrow \mathcal{G} \text { in } \mathbb{Z}^{d} \backslash \tilde{C}^{(u, v)}(v)\right\} \\
& \cap\{\{u, v\} \text { occupied }\} \\
& \cap\{v \Leftrightarrow \mathcal{G} \text { after }\{u, v\} \text { made vacant }\} .
\end{aligned}
$$

Exercise 9.18. Prove that $F_{(u, v)}$ is equal to the above event.

The bond $\{u, v\}$ has an endpoint in $\tilde{C}^{(u, v)}(v)$, and hence the event that $\{u, v\}$ is occupied is independent of the other events above. Therefore,

$$
\begin{array}{r}
\mathbb{P}_{p, \gamma}\left(F_{(u, v)}\right)=p \sum_{A: A \ni v} \mathbb{E}_{p, \gamma}\left[I\left[\tilde{C}^{(u, v)}(v)=A\right] \tilde{I}^{\{u, v\}}[v \Leftrightarrow \mathcal{G}]\right. \\
\left.\times I\left[(0 \leftrightarrow u \text { and } 0 \leftrightarrow \mathcal{G}) \text { in } \mathbb{Z}^{d} \backslash A\right]\right] .
\end{array}
$$


The two events in the first line depend only on bonds with an endpoint in $A$ (but not on $\{u, v\}$ ) and vertices in $A$, while those in the second line depend only on bonds with no endpoint in $A$ (so not on $\{u, v\}$ ) and on vertices in $\mathbb{Z}^{d} \backslash A$. Therefore,

$$
\mathbb{P}_{p, \gamma}\left(F_{(u, v)}\right)=p \sum_{A: A \ni v} \mathbb{E}_{p, \gamma}\left[I\left[\tilde{C}^{(u, v)}(v)=A\right] \tilde{I}^{\{u, v\}}[v \Leftrightarrow \mathcal{G}]\right] \tau_{p, \gamma}^{A}(0, u),
$$

which implies the desired result.

Proof of Proposition 9.15. We use the identities

$$
\tau_{p, \gamma}^{\tilde{C}^{(u, v)}(v)}(0, u)=\tau_{p, \gamma}(0, u)-\left(\tau_{p, \gamma}(0, u)-\tau_{p, \gamma}^{\tilde{C}^{(u, v)}(v)}(0, u)\right)
$$

and

$$
\tilde{I}^{\{u, v\}}[v \Leftrightarrow \mathcal{G}]=I[v \Leftrightarrow \mathcal{G}]-\left(I[v \Leftrightarrow \mathcal{G}]-\tilde{I}^{\{u, v\}}[v \Leftrightarrow \mathcal{G}]\right) .
$$

Recalling (9.90), it follows from Lemma 9.17 that

$$
\begin{aligned}
\mathbb{P}_{p, \gamma}(F)= & p|\Omega| \chi(p, \gamma) \mathbb{P}_{p, \gamma}(0 \Leftrightarrow \mathcal{G}) \\
& -p \sum_{(u, v)} \tau_{p, \gamma}(0, u) \mathbb{E}_{p, \gamma}\left[I[v \Leftrightarrow \mathcal{G}]-\tilde{I}^{\{u, v\}}[v \Leftrightarrow \mathcal{G}]\right] \\
& -p \sum_{(u, v)} \mathbb{E}_{p, \gamma}\left[\left(\tau_{p, \gamma}(0, u)-\tau_{p, \gamma}^{\tilde{C}^{(u, v)}(v)}(0, u)\right) \tilde{I}^{\{u, v\}}[v \Leftrightarrow \mathcal{G}]\right] .
\end{aligned}
$$

We write (9.97) as $X_{1}-X_{2}-X_{3}$, bound $X_{1}$ from below, and bound $X_{2}$ and $X_{3}$ from above.

Lower bound on $X_{1}$. We will prove that

$$
\mathbb{P}_{p, \gamma}(0 \Leftrightarrow \mathcal{G}) \geq\left(\begin{array}{c}
|\Omega| \\
2
\end{array}\right) p^{2}(1-p)^{|\Omega|-2} M(p, \gamma)^{2}\left[(1-\overline{\mathbf{T}}(p))^{2}-\overline{\mathbf{T}}(p)\right] .
$$

The factor $\left(\begin{array}{c}|\Omega| \\ 2\end{array}\right) p^{2}$ is bounded below by a universal constant, since $p|\Omega| \geq 1$. Also, setting setting $p_{1}=0$ and $p_{2}=p_{c}$ in (9.57) gives $p_{c}|\Omega| \leq\left[1-\overline{\mathrm{T}}\left(p_{c}\right)\right]^{-1}$, and therefore the factor $(1-p)^{|\Omega|-2}$ is bounded below by a universal constant. The final factor, in square brackets, is as close to 1 as desired, for $\overline{\mathbf{T}}\left(p_{c}\right)$ sufficiently small, and thus there is a universal constant $a$ such that

$$
X_{1} \geq p|\Omega| \chi(p, \gamma) a M(p, \gamma)^{2} .
$$

To prove (9.98), we first note that the event $\{0 \Leftrightarrow \mathcal{G}\}$ contains the event $\cup_{e, f} E_{e, f}$, where the union is over unordered pairs of vertices $e, f \in \Omega$, the union is disjoint, and the event $E_{e, f}$ is defined as follows. Let $E_{e, f}$ be the event that the bonds $\{0, e\}$ and $\{0, f\}$ are occupied, all other bonds incident on 0 are vacant, and that in the reduced graph $\mathbb{Z}_{-}^{d}$ obtained by deleting the 
origin and each of the $|\Omega|$ bonds incident on 0 from $\mathbb{Z}^{d}$, the following three events occur: $e \leftrightarrow \mathcal{G}, f \leftrightarrow \mathcal{G}$, and $C(e) \cap C(f)=\varnothing$. Let $\mathbb{P}_{p, \gamma}^{-}$denote the joint bond/vertex measure on $\mathbb{Z}_{-}^{d}$. Then

$$
\begin{aligned}
\mathbb{P}_{p, \gamma}(0 \Leftrightarrow \mathcal{G}) & \geq \mathbb{P}_{p, \gamma}\left(\cup_{e, f} E_{e, f}\right)=\sum_{e, f} \mathbb{P}_{p, \gamma}\left(E_{e, f}\right) \\
& =p^{2}(1-p)^{|\Omega|-2} \sum_{e, f} \mathbb{P}_{p, \gamma}^{-}(e \leftrightarrow \mathcal{G}, f \leftrightarrow \mathcal{G}, C(e) \cap C(f)=\varnothing) .
\end{aligned}
$$

Let $W$ denote the event whose probability appears on the right hand side of (9.100). Conditioning on the set $C(e)=A \subset \mathbb{Z}_{-}^{d}$, we see that

$$
\mathbb{P}_{p, \gamma}^{-}(W)=\sum_{A: A \ni e} \mathbb{P}_{p, \gamma}^{-}(C(e)=A, \quad e \leftrightarrow \mathcal{G}, \quad f \leftrightarrow \mathcal{G}, \quad A \cap C(f)=\varnothing) .
$$

The above can be rewritten as

$$
\mathbb{P}_{p, \gamma}^{-}(W)=\sum_{A: A \ni e} \mathbb{P}_{p, \gamma}^{-}\left(C(e)=A, \quad e \leftrightarrow \mathcal{G}, \quad f \leftrightarrow \mathcal{G} \text { in } \mathbb{Z}_{-}^{d} \backslash A\right),
$$

where $\left\{f \leftrightarrow \mathcal{G}\right.$ in $\left.\mathbb{Z}_{-}^{d} \backslash A\right\}$ is the event that there exists $x \in \mathcal{G}$ such that $f \leftrightarrow x$ in $\mathbb{Z}_{-}^{d} \backslash A$. The intersection of the first two events on the right hand side of (9.102) is independent of the third event, and hence

$$
\mathbb{P}_{p, \gamma}^{-}(W)=\sum_{A: A \ni e} \mathbb{P}_{p, \gamma}^{-}(C(e)=A, \quad e \leftrightarrow \mathcal{G}) \mathbb{P}_{p, \gamma}^{-}\left(f \leftrightarrow \mathcal{G} \text { in } \mathbb{Z}_{-}^{d} \backslash A\right) .
$$

Let $M^{-}(x)=\mathbb{P}_{p, \gamma}^{-}(x \leftrightarrow \mathcal{G})$, for $x \in \mathbb{Z}_{-}^{d}$. Then, by the BK inequality and the fact that the two-point function on $\mathbb{Z}_{-}^{d}$ is bounded above by the two-point function on $\mathbb{Z}^{d}$,

$$
\begin{aligned}
\mathbb{P}_{p, \gamma}^{-}\left(f \leftrightarrow \mathcal{G} \text { in } \mathbb{Z}_{-}^{d} \backslash A\right) & =M^{-}(f)-\mathbb{P}_{p, \gamma}^{-}(f \stackrel{A}{\leftrightarrow} \mathcal{G}) \\
& \geq M^{-}(f)-\sum_{y \in A} \tau_{p, 0}(f, y) M^{-}(y) .
\end{aligned}
$$

By definition and the BK inequality,

$$
\begin{aligned}
M^{-}(x) & =M(p, \gamma)-\mathbb{P}_{p, \gamma}(x \stackrel{\{0\}}{\longleftrightarrow} \mathcal{G}) \\
& \geq M(p, \gamma)\left(1-\tau_{p, 0}(0, x)\right) \geq M(p, \gamma)(1-\overline{\mathbf{T}}(p)) .
\end{aligned}
$$

In the above, we also used $\tau_{p, 0}(0, x) \leq \nabla_{p}(x) \leq \overline{\mathbf{T}}(p)$, which follows from (9.51) and (9.54).

It follows from (9.103)-(9.105) and the upper bound $M^{-}(x) \leq M$ that

$$
\begin{aligned}
\mathbb{P}_{p, \gamma}^{-}(W) \geq & M(p, \gamma) \\
& \times \sum_{A: A \ni e} \mathbb{P}_{p, \gamma}^{-}(C(e)=A, \quad e \leftrightarrow \mathcal{G})\left[1-\overline{\mathbf{T}}(p)-\sum_{y \in A} \tau_{p, 0}(f, y)\right] \\
= & M(p, \gamma)\left[M^{-}(e)(1-\overline{\mathbf{T}}(p))-\sum_{y \in \mathbb{Z}_{-}^{d}} \tau_{p, 0}(f, y) \mathbb{P}_{p, \gamma}^{-}(e \leftrightarrow y, e \leftrightarrow \mathcal{G})\right] .
\end{aligned}
$$


By the BK inequality,

$$
\mathbb{P}_{p, \gamma}^{-}(e \leftrightarrow y, e \leftrightarrow \mathcal{G}) \leq \sum_{w \in \mathbb{Z}_{-}^{d}} \tau_{p, 0}(e, w) \tau_{p, 0}(w, y) M^{-}(w),
$$

and hence, by (9.105)-(9.106),

$$
\begin{aligned}
\mathbb{P}_{p, \gamma}^{-}(W) \geq & M(p, \gamma) \\
& \times\left[M^{-}(e)(1-\overline{\mathbf{T}}(p))-\sum_{y, w \in \mathbb{Z}_{-}^{d}} \tau_{p, 0}(f, y) \tau_{p, 0}(e, w) \tau_{p, 0}(w, y) M^{-}(w)\right] \\
\geq & M(p, \gamma)^{2}\left[(1-\overline{\mathbf{T}}(p))^{2}-\overline{\mathbf{T}}(p)\right] .
\end{aligned}
$$

This completes the proof of (9.98), and hence of (9.99).

Upper bound on $X_{2}$. By definition,

$$
X_{2}=p \sum_{(u, v)} \tau_{p, \gamma}(0, u) \mathbb{E}_{p, \gamma}\left[I[v \Leftrightarrow \mathcal{G}]-\tilde{I}^{\{u, v\}}[v \Leftrightarrow \mathcal{G}]\right] .
$$

For the difference of indicators to be nonzero, the double connection from $v$ to $\mathcal{G}$ must be realized via the bond $\{u, v\}$, which therefore must be occupied. The difference of indicators is therefore bounded above by the indicator that the events $\{v \leftrightarrow \mathcal{G}\},\{u \leftrightarrow \mathcal{G}\}$ and $\{\{u, v\}$ occupied $\}$ occur disjointly. Thus we have

$$
\mathbb{E}_{p, \gamma}\left[I[v \Leftrightarrow \mathcal{G}]-\tilde{I}^{\{u, v\}}[v \Leftrightarrow \mathcal{G}]\right] \leq p M(p, \gamma)^{2},
$$

and hence

$$
X_{2} \leq p^{2}|\Omega| M(p, \gamma)^{2} \chi(p, \gamma)
$$

But for $v \in \Omega$,

$$
p \leq \tau_{p}(v) \leq \nabla_{p}(v) \leq \overline{\mathbf{T}}(p) \leq \overline{\mathbf{T}}\left(p_{c}\right),
$$

and hence $X_{2}$ is negligibly small compared to the lower bound (9.99) on $X_{1}$. Upper bound on $X_{3}$. By definition,

$$
X_{3}=p \sum_{(u, v)} \mathbb{E}_{p, \gamma}\left[\left(\tau_{p, \gamma}(0, u)-\tau_{p, \gamma}^{\tilde{C}^{(u, v)}(v)}(0, u)\right) \tilde{I}^{\{u, v\}}[v \Leftrightarrow \mathcal{G}]\right] .
$$

The difference of two-point functions is the expectation of

$$
\begin{aligned}
& I[0 \leftrightarrow u, 0 \leftrightarrow \mathcal{G}]-I\left[0 \leftrightarrow u \text { in } \mathbb{Z}^{d} \backslash \tilde{C}^{(u, v)}(v), 0 \leftrightarrow \mathcal{G}\right] \\
& +I\left[0 \leftrightarrow u \text { in } \mathbb{Z}^{d} \backslash \tilde{C}^{(u, v)}(v), 0 \leftrightarrow \mathcal{G}\right]-I\left[(0 \leftrightarrow u, 0 \leftrightarrow \mathcal{G}) \text { in } \mathbb{Z}^{d} \backslash \tilde{C}^{(u, v)}(v)\right] \\
& \quad \leq I\left[0 \stackrel{\tilde{C}^{(u, v)}(v)}{\longleftrightarrow} u, 0 \nLeftarrow \mathcal{G}\right],
\end{aligned}
$$

since the second line is non-positive and the first line equals the third line. Since the indicator in (9.113) is bounded above by $I[v \Leftrightarrow \mathcal{G}]$, it follows that 


$$
X_{3} \leq p \sum_{(u, v)} \mathbb{E}_{p, \gamma}\left[\mathbb{P}_{p, \gamma}\left(0 \stackrel{\tilde{C}^{(u, v)}(v)}{\longrightarrow} u, 0 \nLeftarrow \mathcal{G}\right) I[v \Leftrightarrow \mathcal{G}]\right]
$$

To estimate the probability within the expectation, we first note that

$$
\mathbb{P}_{p, \gamma}[0 \stackrel{A}{\leftrightarrow} u, 0 \leftrightarrow \mathcal{G}] \leq \sum_{y \in \mathbb{Z}^{d}} \mathbb{P}_{p, \gamma}(\{0 \leftrightarrow y\} \circ\{y \leftrightarrow u\}, 0 \leftrightarrow \mathcal{G}) I[y \in A] .
$$

By conditioning on $\mathcal{G}$, it can be shown that

$$
\mathbb{P}_{p, \gamma}(\{0 \leftrightarrow y\} \circ\{y \leftrightarrow u\}, 0 \leftrightarrow \mathcal{G}) \leq \tau_{p, \gamma}(0, y) \tau_{p, 0}(y, u)
$$

(see [104, Lemma 4.3] for further discussion). The important point in (9.117) is that the condition $0 \nleftarrow \mathcal{G}$ on the left hand side is retained in the factor $\tau_{p, \gamma}(0, y)$ on the right hand side (but not in $\tau_{p, 0}(y, u)$ ). With $(9.115)$, this gives

$$
X_{3} \leq p \sum_{(u, v)} \sum_{y \in \mathbb{Z}^{d}} \tau_{p, \gamma}(0, y) \tau_{p, 0}(y, u) \mathbb{E}_{p, \gamma}\left[I[v \Leftrightarrow \mathcal{G}] I\left[y \in \tilde{C}^{(u, v)}(v)\right]\right] .
$$

A further application of BK gives

$$
\begin{aligned}
X_{3} & \leq p \sum_{y \in \mathbb{Z}^{d}} \tau_{p, \gamma}(0, y) \sum_{(u, v)} \tau_{p, 0}(y, u) \sum_{w \in \mathbb{Z}^{d}} \tau_{p, 0}(v, w) \tau_{p, 0}(y, w) M(p, \gamma)^{2} \\
& \leq p M(p, \gamma)^{2} \chi(p, \gamma)|\Omega| \overline{\mathrm{T}}(p)
\end{aligned}
$$

using symmetry in the last step. Since $\mathrm{T}\left(p_{c}\right)$ may be assumed to be as small as desired, the upper bound (9.119) is negligibly small compared to the lower bound (9.99) on $X_{1}$.

The combination of $(9.99),(9.111)$ and (9.119) completes the proof of (9.87).

\section{The Expansion for Percolation}

In Section 10.1, we derive the expansion for percolation. The expansion produces quantities $\Pi^{(N)}$, and we show in Section 10.2 how these quantities can be bounded by Feynman diagrams. Finally, we show in Section 10.3 how the diagrams can be bounded in terms of the triangle diagram. The methods of this section are due to [94], but we follow the exposition of [32], often verbatim.

We do not prove convergence of the expansion here. Convergence proofs can be found in [32, 91, 94], all based on the bootstrap Lemma 5.9.

\subsection{The Expansion}

The expansions discussed in Sections 3 and 8, for the self-avoiding walk, lattice trees, and lattice animals, each have dual interpretations as arising either from 
repeated inclusion-exclusion or from expansion of an interaction $\prod_{s t}\left(1+U_{s t}\right)$ followed by resummation involving laces. For percolation, however, only the inclusion-exclusion approach has been used. (For the case of oriented percolation, laces can be used; see Section 13.2.) In this section, we derive the expansion using repeated inclusion-exclusion. The expansion applies to any graph, finite or infinite, and we will derive the expansion in the setting of an arbitrary connected finite or infinite graph $\mathbb{G}$, which need not even be transitive or regular. ${ }^{9}$

Let $\mathbb{G}=(\mathbb{V}, \mathbb{B})$ be an arbitrary connected graph, finite or infinite, with vertex set $\mathbb{V}$ and edge set $\mathbb{B}$. We consider bond percolation on $\mathbb{G}$. Given $p \in$ $[0,1]$, we define

$$
\begin{aligned}
J(x, y) & =p I[\{x, y\} \in \mathbb{B}] \\
& =p|\Omega| D(x, y) \text { if } \mathbb{G} \text { is regular, }
\end{aligned}
$$

with $D(x, y)$ equal to the reciprocal of the degree $|\Omega|$ times the indicator that $\{x, y\}$ is an edge. We write $\tau(x, y)=\tau_{p}(x, y)$ for brevity, and generally drop subscripts indicating dependence on $p$.

Given a percolation cluster containing 0 and $x$, we call the connected components that remain after removing all the pivotal bonds for $0 \leftrightarrow x$ sausages (vertices are not removed when the bonds are removed). See Fig. 10.1. Since they are separated by at least one pivotal bond by definition, no two sausages can have a common vertex. Thus, the sausages are constrained to be mutually avoiding. However, this is a weak constraint, since sausage intersections require a cycle, and cycles are unlikely in high dimensions. The fact that cycles are unlikely also means that sausages tend to be trees. This makes it reasonable to attempt to apply an inclusion-exclusion analysis, where the connection from 0 to $x$ is treated as a random walk path, with correction terms taking into account cycles in sausages and the avoidance constraint between sausages.
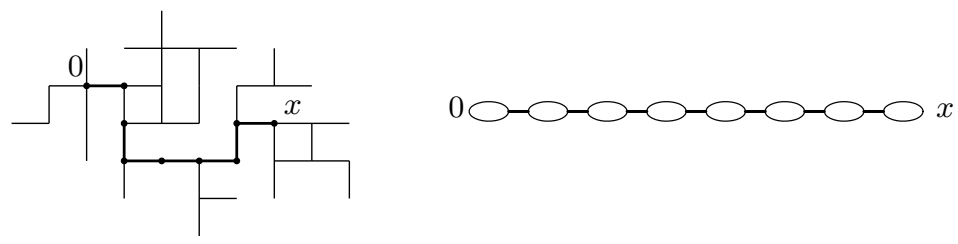

Fig. 10.1. A percolation cluster with a string of 8 sausages joining 0 to $x$, and a schematic representation of the string of sausages. The 7 pivotal bonds are shown in bold.

The inclusion-exclusion expansion of [94] makes this procedure precise. For each $M=0,1,2, \ldots$, the expansion takes the form

\footnotetext{
${ }^{9} \mathrm{~A}$ graph is regular if very vertex has the same degree. A transitive graph is defined in Section 11.4.2.
} 


$$
\tau(x, y)=\delta_{x, y}+(J * \tau)(x, y)+\left(\Pi_{M} * J * \tau\right)(x, y)+\Pi_{M}(x, y)+R_{M}(x, y),
$$

where the $*$ product denotes matrix multiplication (this reduces to convolution when $\mathbb{G}=\mathbb{Z}^{d}$ and the graph is translation invariant). The function $\Pi_{M}: \mathbb{V} \times$ $\mathbb{V} \rightarrow \mathbb{R}$ is the central quantity in the expansion, and $R_{M}(x, y)$ is a remainder term. The dependence of $\Pi_{M}$ on $M$ is given by

$$
\Pi_{M}(x, y)=\sum_{N=0}^{M}(-1)^{N} \Pi^{(N)}(x, y)
$$

with $\Pi^{(N)}(x, y)$ independent of $M$. The alternating sign in (10.3) arises via repeated inclusion-exclusion. When the expansion converges, one has

$$
\lim _{M \rightarrow \infty} \sum_{y}\left|R_{M}(x, y)\right|=0 .
$$

This leads to

$$
\tau(x, y)=\delta_{x, y}+(J * \tau)(x, y)+(\Pi * J * \tau)(x, y)+\Pi(x, y),
$$

with $\Pi=\Pi_{\infty}$ (cf. (8.11) for lattice trees). For a translation invariant bond percolation model on $\mathbb{Z}^{d}$, taking the Fourier transform of (10.5) and solving for $\hat{\tau}(k)$ gives

$$
\hat{\tau}(k)=\frac{1+\hat{\Pi}(k)}{1-p|\Omega| \hat{D}(k)[1+\hat{\Pi}(k)]} .
$$

This is similar to the formula (3.30) for the self-avoiding walk, but not identical.

The remainder of this section gives the proof of (10.2). Recall Definition 9.13. Also, given a bond configuration, we say that $x$ is doubly connected to $y$, and we write $x \Leftrightarrow y$, if $x=y$ or if there are at least two bond-disjoint paths from $x$ to $y$ consisting of occupied bonds. We denote by $P_{(x, y)}$ the set of directed pivotal bonds for the connection from $x$ to $y$.

To begin the expansion, we define

$$
\Pi^{(0)}(x, y)=\mathbb{P}(x \Leftrightarrow y)-\delta_{x, y}
$$

and distinguish configurations with $x \leftrightarrow y$ according to whether or not there is a double connection, to obtain

$$
\tau(x, y)=\delta_{x, y}+\Pi^{(0)}(x, y)+\mathbb{P}(x \leftrightarrow y, x \nLeftarrow y) .
$$

If $x$ is connected to $y$, but not doubly, then $P_{(x, y)}$ is nonempty. There is therefore a unique element $(u, v) \in P_{(x, y)}$ (the first pivotal bond) such that $x \Leftrightarrow u$, and we can write

$$
\mathbb{P}(x \leftrightarrow y, x \nLeftarrow y)=\sum_{(u, v)} \mathbb{P}\left(x \Leftrightarrow u, \quad(u, v) \text { occupied },(u, v) \in P_{(x, y)}\right) .
$$


Now comes the essential part of the expansion. Ideally, we would like to factor the probability on the right hand side of (10.9) as

$$
\mathbb{P}(x \Leftrightarrow u) \mathbb{P}((u, v) \text { is occupied }) \mathbb{P}(v \leftrightarrow y)=\left(\delta_{x, u}+\Pi^{(0)}(x, u)\right) J(u, v) \tau(v, y) .
$$

The expression (10.10) leads to (10.2) with $\Pi_{M}=\Pi^{(0)}$ and $R_{M}=0$. However, (10.9) does not factor in this way because the cluster $\tilde{C}^{(u, v)}(u)$ is constrained not to intersect the cluster $\tilde{C}^{(u, v)}(v)$, since $(u, v)$ is pivotal. What we can do is approximate the probability on the right hand side of (10.9) by (10.10), and then attempt to deal with the error term.

For this, we will use the next lemma, which gives an identity for the probability on the right hand side of (10.9). In fact, we will also need a more general identity, involving the following generalizations of the event appearing on the right hand side of (10.9). Let $x, u, v, y \in \mathbb{V}$, and let $A \subset \mathbb{V}$ be nonempty. Then we define the events

$$
E^{\prime}(v, y ; A)=\{v \stackrel{A}{\leftrightarrow} y\} \cap\left\{\nexists\left(u^{\prime}, v^{\prime}\right) \in P_{(v, y)} \text { such that } v \stackrel{A}{\leftrightarrow} u^{\prime}\right\}
$$

and

$$
E(x, u, v, y ; A)=E^{\prime}(x, u ; A) \cap\{(u, v) \text { is occupied and pivotal for } x \leftrightarrow y\} .
$$

Note that $\{x \Leftrightarrow y\}=E^{\prime}(x, y ; \mathbb{V})$, while $E(x, u, v, y ; \mathbb{V})$ is the event appearing on the right hand side of (10.9). A version of Lemma 10.1, with $E^{\prime}(x, u ; A)$ replaced by $\{0 \leftrightarrow u\}$ on both sides of (10.13), appeared in (9.78).

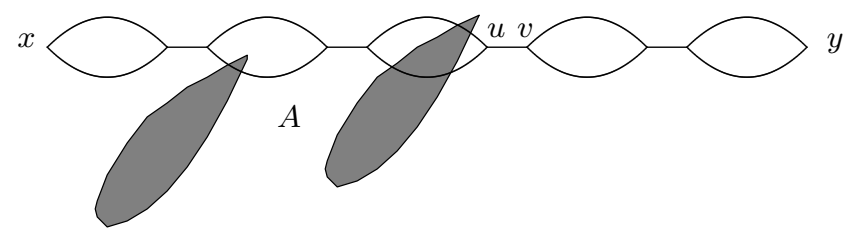

Fig. 10.2. The event $E(x, u, v, y ; A)$ of Lemma 10.1. The shaded regions represent the vertices in $A$. Intersection between $A$ and sausages to the right of $(u, v)$ is permitted.

Lemma 10.1. Let $\mathbb{G}$ be a finite or an infinite graph, let $p \in[0,1]$ be such that there is almost surely no infinite cluster, let $u \in \mathbb{V}$, and let $A \subset \mathbb{V}$ be nonempty. Then

$$
\mathbb{E}(I[E(x, u, v, y ; A)])=p \mathbb{E}\left(I\left[E^{\prime}(x, u ; A)\right] \tau^{\tilde{C}^{\{u, v\}}(x)}(v, y)\right) .
$$

Proof. The event appearing in the left hand side of (10.13) is depicted in Fig. 10.2. We first observe that the event $E^{\prime}(x, u ; A) \cap\left\{(u, v) \in P_{(x, y)}\right\}$ is 
independent of the occupation status of the bond $(u, v)$. This is true by definition for $\left\{(u, v) \in P_{(x, y)}\right\}$, and when $(u, v)$ is pivotal, the occurrence or not of $E^{\prime}(x, u ; A)$ cannot be affected by $\{u, v\}$ since $E^{\prime}(x, u ; A)$ is determined by the occupied paths from $x$ to $u$ and in this case no such path uses the bond $\{u, v\}$. Therefore, the left hand side of the identity in the statement of the lemma is equal to

$$
p \mathbb{E}\left(I\left[E^{\prime}(x, u ; A) \cap\left\{(u, v) \in P_{(x, y)}\right\}\right]\right) .
$$

By conditioning on $\tilde{C}^{\{u, v\}}(x),(10.14)$ is equal to

$$
p \sum_{S: S \ni x} \mathbb{E}\left(I\left[E^{\prime}(x, u ; A) \cap\left\{(u, v) \in P_{(x, y)}\right\} \cap\left\{\tilde{C}^{\{u, v\}}(x)=S\right\}\right]\right),
$$

where the sum is over all finite connected sets of vertices $S$ containing $x$.

In (10.15), we can replace

$$
\left\{(u, v) \in P_{(x, y)}\right\} \cap\left\{\tilde{C}^{\{u, v\}}(x)=S\right\}
$$

by

$$
\{v \leftrightarrow y \text { in } \mathbb{V} \backslash S\} \cap\left\{\tilde{C}^{\{u, v\}}(x)=S\right\} .
$$

The event $\{v \leftrightarrow y$ in $\mathbb{V} \backslash S\}$ depends only on the occupation status of bonds which do not have an endpoint in $S$. On the other hand, when $\{v \leftrightarrow y$ in $\mathbb{V} \backslash S\} \cap\left\{\tilde{C}^{\{u, v\}}(x)=S\right\}$ occurs, the event $E^{\prime}(x, u ; A)$ is determined by the occupation status of bonds which have an endpoint in $S=\tilde{C}^{\{u, v\}}(x)$. Similarly, the event $\left\{\tilde{C}^{\{u, v\}}(x)=S\right\}$ depends on bonds which have one or both endpoints in $S$. Hence, given $S$, the event $E^{\prime}(x, u ; A) \cap\left\{\tilde{C}^{\{u, v\}}(x)=S\right\}$ is independent of the event $\{v \leftrightarrow y$ in $\mathbb{V} \backslash S\}$, and therefore (10.15) is equal to

$$
p \sum_{S: S \ni x} \mathbb{E}\left(I\left[E^{\prime}(x, u ; a) \cap\left\{\tilde{C}^{\{u, v\}}(x)=S\right\}\right]\right) \tau_{p}^{S}(v, y) .
$$

Bringing the restricted two-point function inside the expectation, replacing the superscript $S$ by $\tilde{C}^{\{u, v\}}(x)$, and performing the sum over $S$, gives the desired result.

It follows from (10.9) and Lemma 10.1 that

$$
\mathbb{P}(x \leftrightarrow y, x \nLeftarrow y)=\sum_{(u, v)} J(u, v) \mathbb{E}\left(I[x \Leftrightarrow u] \tau^{\tilde{C}^{(u, v)}(x)}(v, y)\right) .
$$

On the right hand side, $\tau^{\tilde{C}^{(u, v)}(x)}(v, y)$ is the restricted two-point function given the cluster $\tilde{C}^{(u, v)}(x)$ of the expectation $\mathbb{E}$, so that in the expectation defining $\tau^{\tilde{C}^{(u, v)}(x)}(v, y), \tilde{C}^{(u, v)}(x)$ should be regarded as a fixed set. The restricted two-point function effectively introduces a second percolation model on a second graph, as in (9.78).

We write 


$$
\begin{aligned}
\tau^{\tilde{C}^{(u, v)}(x)}(v, y) & =\tau(v, y)-\left(\tau(v, y)-\tau^{\tilde{C}^{(u, v)}(x)}(v, y)\right) \\
& =\tau(v, y)-\mathbb{P}\left(v \stackrel{\tilde{C}^{(u, v)}(x)}{\longrightarrow} y\right),
\end{aligned}
$$

insert this into (10.19), and use (10.8) and (10.7) to obtain

$$
\begin{aligned}
\tau(x, y)= & \delta_{x, y}+\Pi^{(0)}(x, y)+\sum_{(u, v)}\left(\delta_{x, u}+\Pi^{(0)}(x, u)\right) J(u, v) \tau(v, y) \\
& -\sum_{(u, v)} J(u, v) \mathbb{E}\left(I[x \Leftrightarrow u] \mathbb{P}\left(v \stackrel{\tilde{C}^{(u, v)}(x)}{\longleftrightarrow} y\right)\right) .
\end{aligned}
$$

With $R_{0}(x, y)$ equal to the last term on the right hand side of (10.21) (including the minus sign), this proves (10.2) for $M=0$.

To continue the expansion, we would like to rewrite the final term of (10.21) in terms of a product with the two-point function. A configuration contributing to the expectation in the final term of (10.21) is illustrated schematically in Fig. 10.3, in which the bonds drawn with heavy lines should be regarded as living on a different graph than the bonds drawn with lighter lines. Our goal is to extract a factor $\tau\left(v^{\prime}, y\right)$, where $v^{\prime}$ is shown in Fig. 10.3.

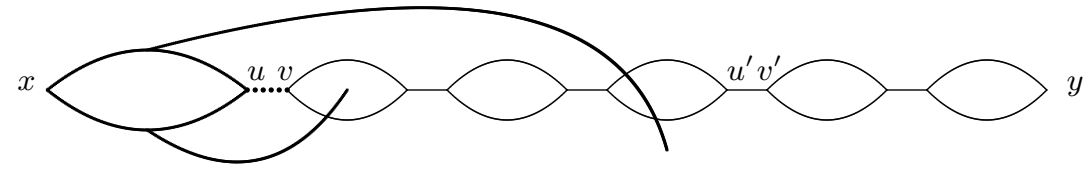

Fig. 10.3. A possible configuration appearing in the second stage of the expansion.

Given a configuration in which $v \stackrel{A}{\longleftrightarrow} y$, the cutting bond $\left(u^{\prime}, v^{\prime}\right)$ is defined to be the first pivotal bond for $v \leftrightarrow y$ such that $v \stackrel{A}{\longleftrightarrow} u^{\prime}$. It is possible that no such bond exists, as for example would be the case in Fig. 10.3 if only the leftmost four sausages were included in the figure, with $y$ in the location currently occupied by $u^{\prime}$. Recall the definitions of $E^{\prime}(v, y ; A)$ and $E(x, u, v, y ; A)$ in $(10.11)$ and $(10.12)$. By partitioning $\{v \stackrel{A}{\leftrightarrow} y\}$ according to the location of the cutting bond (or the lack of a cutting bond), we obtain the partition

$$
\{v \stackrel{A}{\leftrightarrow} y\}=E^{\prime}(v, y ; A) \bigcup \bigcup_{\left(u^{\prime}, v^{\prime}\right)} E\left(v, u^{\prime}, v^{\prime}, y ; A\right)
$$

which implies that

$$
\mathbb{P}(v \stackrel{A}{\leftrightarrow} y)=\mathbb{P}\left(E^{\prime}(v, y ; A)\right)+\sum_{\left(u^{\prime}, v^{\prime}\right)} \mathbb{P}\left(E\left(v, u^{\prime}, v^{\prime}, y ; A\right)\right) .
$$


Using Lemma 10.1, this gives

$$
\begin{aligned}
\mathbb{P}(v \stackrel{A}{\leftrightarrow} y)= & \mathbb{P}\left(E^{\prime}(v, y ; A)\right) \\
& +\sum_{\left(u^{\prime}, v^{\prime}\right)} J\left(u^{\prime}, v^{\prime}\right) \mathbb{E}\left(I\left[E^{\prime}\left(v, u^{\prime} ; A\right)\right] \tau^{\tilde{C}^{\left(u^{\prime}, v^{\prime}\right)}(v)}\left(v^{\prime}, y\right)\right) .
\end{aligned}
$$

Inserting the identity (10.20) into (10.24), we obtain

$$
\begin{aligned}
\mathbb{P}(v \stackrel{A}{\leftrightarrow} y)= & \mathbb{P}\left(E^{\prime}(v, y ; A)\right)+\sum_{\left(u^{\prime}, v^{\prime}\right)} J\left(u^{\prime}, v^{\prime}\right) \mathbb{P}\left(E^{\prime}\left(v, u^{\prime} ; A\right)\right) \tau\left(v^{\prime}, y\right) \\
& -\sum_{\left(u^{\prime}, v^{\prime}\right)} J\left(u^{\prime}, v^{\prime}\right) \mathbb{E}_{1}\left(I\left[E^{\prime}\left(v, u^{\prime} ; A\right)\right] \mathbb{P}_{2}\left(v^{\prime} \stackrel{\tilde{C}_{1}^{\left(u^{\prime}, v^{\prime}\right)}(v)}{\longleftrightarrow} y\right)\right)
\end{aligned}
$$

In the last term on the right hand side, we have introduced subscripts for $\tilde{C}$ and the expectations, to indicate to which expectation $\tilde{C}$ belongs.

Let

$$
\Pi^{(1)}(x, y)=\sum_{(u, v)} J(u, v) \mathbb{E}_{0}\left(I[x \Leftrightarrow u] \mathbb{P}_{1}\left(E^{\prime}\left(v, y ; \tilde{C}_{0}^{(u, v)}(x)\right)\right)\right) .
$$

Inserting (10.25) into (10.21), and using (10.26), we have

$$
\begin{aligned}
\tau(x, y)= & \delta_{x, y}+\Pi^{(0)}(x, y)-\Pi^{(1)}(x, y) \\
& +\sum_{(u, v)}\left(\delta_{x, u}+\Pi^{(0)}(x, u)-\Pi^{(1)}(x, u)\right) J(u, v) \tau(v, y) \\
& +\sum_{(u, v)} J(u, v) \sum_{\left(u^{\prime}, v^{\prime}\right)} J\left(u^{\prime}, v^{\prime}\right) \\
& \quad \times \mathbb{E}_{0}\left(I[x \Leftrightarrow u] \mathbb{E}_{1}\left(I\left[E^{\prime}\left(v, u^{\prime} ; \tilde{C}_{0}{ }^{(u, v)}(x)\right)\right] \mathbb{P}_{2}\left(v^{\prime} \stackrel{\tilde{C}_{1}^{\left(u^{\prime}, v^{\prime}\right)}(v)}{\longrightarrow} y\right)\right)\right) .
\end{aligned}
$$

This proves (10.2) for $M=1$, with $R_{1}(x, y)$ given by the last two lines of (10.27).

We now repeat this procedure recursively, rewriting $\mathbb{P}_{2}\left(v^{\prime} \stackrel{\tilde{C}_{1}^{\left(u^{\prime}, v^{\prime}\right)}(v)}{\longleftrightarrow} y\right)$ using (10.25), and so on. This leads to (10.2), with $\Pi^{(0)}$ and $\Pi^{(1)}$ given by (10.7) and (10.26), and, for $N \geq 2$, 


$$
\begin{aligned}
\Pi^{(N)}(x, y)= & \sum_{\left(u_{0}, v_{0}\right)} \ldots \sum_{\left(u_{N-1}, v_{N-1}\right)}\left[\prod_{i=0}^{N-1} J\left(u_{i}, v_{i}\right)\right] \mathbb{E}_{0} I\left[x \Leftrightarrow u_{0}\right] \\
& \times \mathbb{E}_{1} I\left[E^{\prime}\left(v_{0}, u_{1} ; \tilde{C}_{0}\right)\right] \cdots \mathbb{E}_{N-1} I\left[E^{\prime}\left(v_{N-2}, u_{N-1} ; \tilde{C}_{N-2}\right)\right] \\
& \times \mathbb{E}_{N} I\left[E^{\prime}\left(v_{N-1}, y ; \tilde{C}_{N-1}\right)\right] \\
R_{M}(x, y)=( & 1)^{M+1} \sum_{\left(u_{0}, v_{0}\right)} \ldots \sum_{\left(u_{M}, v_{M}\right)}\left[\prod_{i=0}^{M} J\left(u_{i}, v_{i}\right)\right] \mathbb{E}_{0} I\left[x \Leftrightarrow u_{0}\right] \\
& \times \mathbb{E}_{1} I\left[E^{\prime}\left(v_{0}, u_{1} ; \tilde{C}_{0}\right)\right] \cdots \mathbb{E}_{M-1} I\left[E^{\prime}\left(v_{M-2}, u_{M-1} ; \tilde{C}_{M-2}\right)\right] \\
& \times \mathbb{E}_{M}\left[I\left[E^{\prime}\left(v_{M-1}, u_{M} ; \tilde{C}_{M-1}\right)\right] \mathbb{P}_{M+1}\left(v_{M} \stackrel{\tilde{C}_{M}}{\longleftrightarrow} y\right)\right], \quad(10.29)
\end{aligned}
$$

where we have used the abbreviation $\tilde{C}_{j}=\tilde{C}_{j}^{\left(u_{j}, v_{j}\right)}\left(v_{j-1}\right)$, with $v_{-1}=x$.

Since

$$
\mathbb{P}_{M+1}\left(v_{M} \stackrel{\tilde{C}_{M}}{\longleftrightarrow} y\right) \leq \tau_{p}\left(v_{M}, y\right)
$$

it follows from (10.28)-(10.29) that

$$
\left|R_{M}(x, y)\right| \leq \sum_{u_{M}, v_{M} \in \mathbb{V}} \Pi^{(M)}\left(x, u_{M}\right) J\left(u_{M}, v_{M}\right) \tau_{p}\left(v_{M}, y\right) .
$$

This bound is typically used to show that the remainder vanishes in the limit $M \rightarrow \infty$, once we can show that $\sum_{u} \Pi^{(M)}(x, u)$ vanishes in the limit $M \rightarrow \infty$.

In deriving the expansion, we have essentially been making an approximation in which $\tau^{\tilde{C}^{\{u, v\}}(0)}(v, x)$ is replaced by $\tau(v, x)$. This is a good approximation when the backbone joining $v$ and $x$ typically does not intersect the cluster $\tilde{C}^{\{u, v\}}(0)$. Above the upper critical dimension, we expect the backbone to behave like Brownian motion, which is 2-dimensional, and the cluster $\tilde{C}^{\{u, v\}}(0)$ to have the dimensionality of an ISE cluster (see Section 16.5 below), which is 4-dimensional. These objects generically do not intersect above dimension $6=4+2$, and this provides an interpretation of the upper critical dimension.

\subsection{The Diagrams}

In this section, we show how $\Pi^{(N)}$ of (10.28) can be bounded in terms of Feynman diagrams. We follow the presentation of [32], and the method is essentially that of [94, Section 2.2]. The results of this section apply to any graph $\mathbb{G}=(\mathbb{V}, \mathbb{B})$, finite or infinite, which need not be transitive nor regular.

Let $\mathbb{P}^{(N)}$ denote the product measure on $N+1$ copies of percolation on G. By Fubini's Theorem and (10.28),

$$
\begin{aligned}
& \Pi^{(N)}(x, y)=\sum_{\left(u_{0}, v_{0}\right)} \ldots \sum_{\left(u_{N-1}, v_{N-1}\right)}\left[\prod_{i=0}^{N-1} J\left(u_{i}, v_{i}\right)\right] \\
& \times \mathbb{P}^{(N)}\left(\left\{x \Leftrightarrow u_{0}\right\}_{0} \cap\left(\bigcap_{i=1}^{N-1} E^{\prime}\left(v_{i-1}, u_{i} ; \tilde{C}_{i-1}\right)_{i}\right) \cap E^{\prime}\left(v_{N-1}, y ; \tilde{C}_{N-1}\right)_{N}\right),
\end{aligned}
$$


where, for an event $F$, we write $F_{i}$ to denote that $F$ occurs on graph $i$. To estimate $\Pi^{(N)}(x, y)$ for $N \geq 1$, it is convenient to define the events

$$
\begin{gathered}
F_{0}\left(x, u_{0}, w_{0}, z_{1}\right)=\left\{x \leftrightarrow u_{0}\right\} \circ\left\{x \leftrightarrow w_{0}\right\} \circ\left\{w_{0} \leftrightarrow u_{0}\right\} \circ\left\{w_{0} \leftrightarrow z_{1}\right\}, \\
\begin{aligned}
F^{\prime}\left(v_{i-1}, t_{i}, z_{i}, u_{i}, w_{i}, z_{i+1}\right)=\left\{v_{i-1} \leftrightarrow t_{i}\right\} \circ\left\{t_{i} \leftrightarrow z_{i}\right\} \circ\left\{t_{i} \leftrightarrow w_{i}\right\} \\
\quad \circ\left\{z_{i} \leftrightarrow u_{i}\right\} \circ\left\{w_{i} \leftrightarrow u_{i}\right\} \circ\left\{w_{i} \leftrightarrow z_{i+1}\right\},
\end{aligned} \\
\begin{aligned}
F^{\prime \prime}\left(v_{i-1}, t_{i}, z_{i}, u_{i}, w_{i}, z_{i+1}\right)=\left\{v_{i-1} \leftrightarrow w_{i}\right\} \circ\left\{w_{i} \leftrightarrow t_{i}\right\} \circ\left\{t_{i} \leftrightarrow z_{i}\right\} \\
\circ\left\{t_{i} \leftrightarrow u_{i}\right\} \circ\left\{z_{i} \leftrightarrow u_{i}\right\} \circ\left\{w_{i} \leftrightarrow z_{i+1}\right\},
\end{aligned} \\
\begin{aligned}
F\left(v_{i-1}, t_{i}, z_{i}, u_{i}, w_{i}, z_{i+1}\right)= & F^{\prime}\left(v_{i-1}, t_{i}, z_{i}, u_{i}, w_{i}, z_{i+1}\right) \\
& \cup F^{\prime \prime}\left(v_{i-1}, t_{i}, z_{i}, u_{i}, w_{i}, z_{i+1}\right), \\
& \\
F_{N}\left(v_{N-1}, t_{N}, z_{N}, y\right)=\left\{v_{N-1} \leftrightarrow\right. & \left.\left.t_{N}\right\} \circ\left\{t_{N} \leftrightarrow z_{N}\right\} \circ\left\{t_{N} \leftrightarrow x\right\} \circ\left\{z_{N} \leftrightarrow y\right\} .36\right)
\end{aligned}
\end{gathered}
$$

The events $F_{0}, F^{\prime}, F^{\prime \prime}, F_{N}$ are depicted in Fig. 10.4. Note that

$$
F_{N}(v, t, z, y)=F_{0}(y, z, t, v) .
$$

By the definition of $E^{\prime}$ in (10.11),

$$
E^{\prime}\left(v_{N-1}, y ; \tilde{C}_{N-1}\right)_{N} \subset \bigcup_{z_{N} \in \tilde{C}_{N-1}} \bigcup_{t_{N} \in \mathbb{V}} F_{N}\left(v_{N-1}, t_{N}, z_{N}, y\right)_{N} .
$$

Indeed, viewing the connection from $v_{N-1}$ to $y$ as a string of sausages beginning at $v_{N-1}$ and ending at $y$, for the event $E^{\prime}$ to occur there must be a vertex $z_{N} \in \tilde{C}_{N-1}$ that lies on the last sausage, on a path from $v_{N-1}$ to $y$. (In fact, both "sides" of the sausage must contain a vertex in $\tilde{C}_{N-1}$, but we do not need or use this.) This leads to (10.39), with $t_{N}$ representing the other endpoint of the sausage that terminates at $y$.

Assume, for the moment, that $N \geq 2$. The condition in (10.39) that $z_{N} \in$ $\tilde{C}_{N-1}$ is a condition on the graph $N-1$ that must be satisfied in conjunction with the event $E^{\prime}\left(v_{N-2}, u_{N-1} ; \tilde{C}_{N-2}\right)_{N-1}$. It is not difficult to see that for $i \in\{1, \ldots, N-1\}$,

$$
E^{\prime}\left(v_{i-1}, u_{i} ; \tilde{C}_{i-1}\right)_{i} \cap\left\{z_{i+1} \in \tilde{C}_{i}\right\} \subset \bigcup_{z_{i} \in \tilde{C}_{i-1}} \bigcup_{t_{i}, w_{i} \in \mathbb{V}} F\left(v_{i-1}, t_{i}, z_{i}, u_{i}, w_{i}, z_{i+1}\right)_{i} .
$$

See Fig. 10.5 for a depiction of the inclusions in (10.39) and (10.40). Informally, for (10.40), any path from $v_{i-1}$ to $u_{i}$ must contain a vertex with a disjoint 


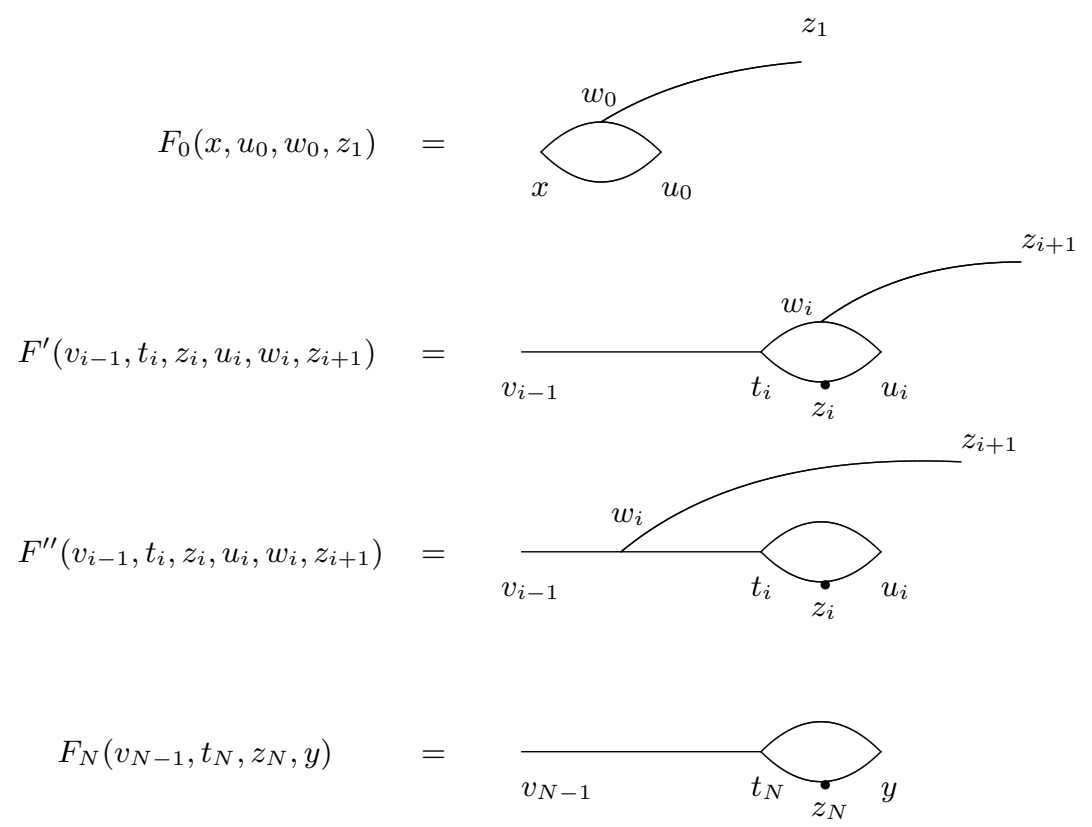

Fig. 10.4. Diagrammatic representations of the events $\left.F_{0}\left(x, u_{0}, w_{0}, z_{1}\right)\right)$, $F^{\prime}\left(v_{i-1}, t_{i}, z_{i}, u_{i}, w_{i}, z_{i+1}\right), F^{\prime \prime}\left(v_{i-1}, t_{i}, z_{i}, u_{i}, w_{i}, z_{i+1}\right), F_{N}\left(v_{N-1}, t_{N}, z_{N}, y\right)$. Lines indicate disjoint connections.

connection to $z_{i+1}$, and this can occur in the two topologically distinct manners illustrated in Fig. 10.5. The definition of $E^{\prime}$ requires the existence of a vertex $z_{i} \in \tilde{C}_{i-1}$ on every path from $t_{i}$ to $u_{i}$, where the latter are the endpoints of the last sausage, and so, in particular, such a vertex can be found on the "other" side of the sausage, in the case where $z_{i+1}$ connects to the last sausage.

With an appropriate treatment for graph $0,(10.39)$ and (10.40) lead to

$$
\begin{aligned}
& \left\{x \Leftrightarrow u_{0}\right\}_{0} \cap\left(\bigcap_{i=1}^{N-1} E^{\prime}\left(v_{i-1}, u_{i} ; \tilde{C}_{i-1}\right)_{i}\right) \cap E^{\prime}\left(v_{N-1}, y ; \tilde{C}_{N-1}\right)_{N} \\
& \subset \bigcup_{\vec{t}, \vec{w}, \vec{z}}\left(F_{0}\left(x, u_{0}, w_{0}, z_{1}\right)_{0} \cap\left(\bigcap_{i=1}^{N-1} F\left(v_{i-1}, t_{i}, z_{i}, u_{i}, w_{i}, z_{i+1}\right)_{i}\right)\right. \\
& \left.\cap F_{N}\left(v_{N-1}, t_{N}, z_{N}, y\right)_{N}\right) \text {, }
\end{aligned}
$$

where $\vec{t}=\left(t_{1}, \ldots, t_{N}\right), \vec{w}=\left(w_{0}, \ldots, w_{N-1}\right)$ and $\vec{z}=\left(z_{1}, \ldots, z_{N}\right)$. Therefore, 

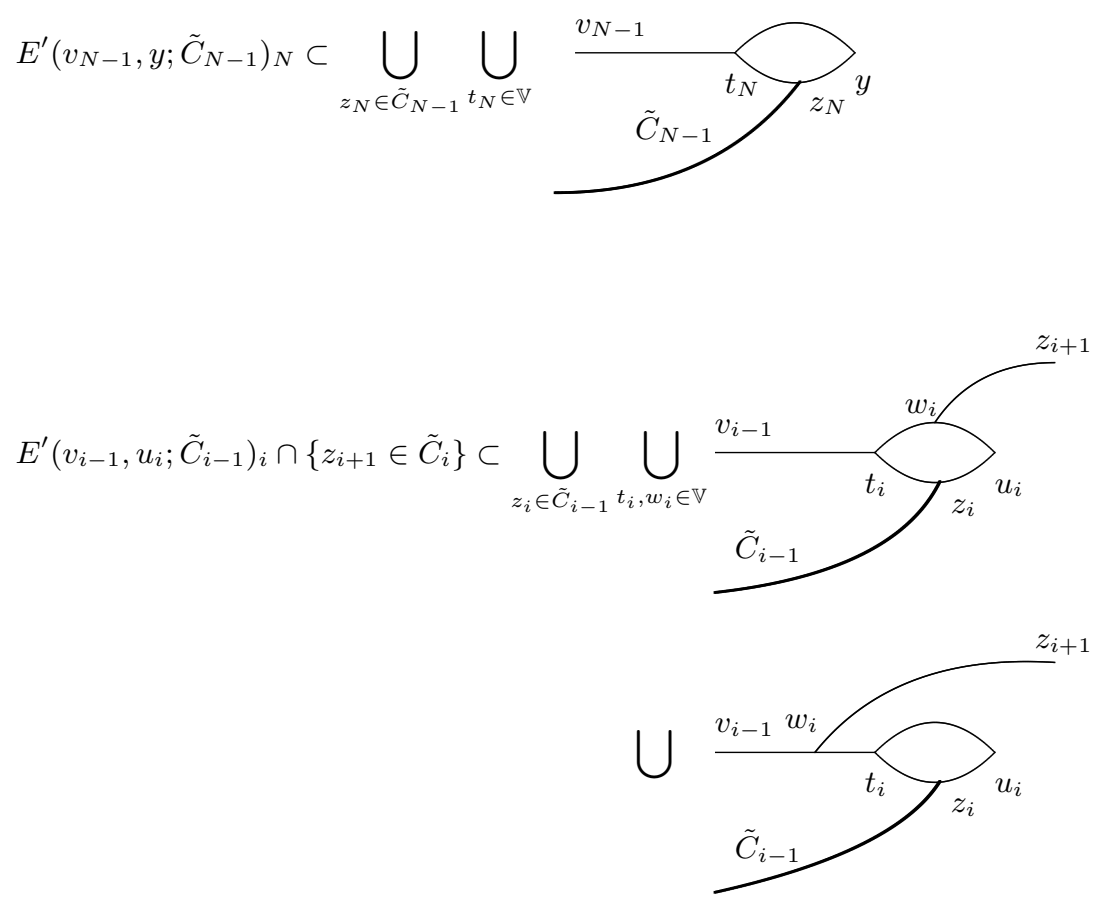

Fig. 10.5. Diagrammatic representations of the inclusions in (10.39) and (10.40).

$$
\begin{aligned}
\Pi^{(N)}(x, y) \leq \sum & {\left[\prod_{i=0}^{N-1} J\left(u_{i}, v_{i}\right)\right] \mathbb{P}_{p}\left(F_{0}\left(x, u_{0}, w_{0}, z_{1}\right)\right) } \\
& \times \prod_{i=1}^{N-1} \mathbb{P}_{p}\left(F\left(v_{i-1}, t_{i}, z_{i}, u_{i}, w_{i}, z_{i+1}\right)\right) \mathbb{P}_{p}\left(F_{N}\left(v_{N-1}, t_{N}, z_{N}, y\right)\right),
\end{aligned}
$$

where the summation is over all vertices $z_{1}, \ldots, z_{N}, t_{1}, \ldots, t_{N}, w_{0}, \ldots, w_{N-1}$, $u_{0}, \ldots, u_{N-1}, v_{0}, \ldots, v_{N-1}$. The probability in (10.43) factors because the events $F_{0}, \ldots, F_{N}$ are events on different percolation models. Each probability in (10.43) can be estimated using the BK inequality. The result is that each of the connections $\{a \leftrightarrow b\}$ present in the events $F_{0}, F$ and $F_{N}$ is replaced by a two-point function $\tau_{p}(a, b)$. This results in a large sum of two-point functions.

To organize a large sum of this form, we let

$$
\begin{aligned}
\tilde{\tau}_{p}(x, y) & =\left(J * \tau_{p}\right)(x, y) \\
& =p|\Omega|\left(D * \tau_{p}\right)(x, y) \text { if } \mathbb{G} \text { is regular, }
\end{aligned}
$$




$$
\begin{aligned}
& A_{3}(s, u, v)=s \bigwedge_{v}^{u} \quad B_{1}(s, t, u, v)=s_{s \longleftarrow}^{t \longmapsto \longmapsto} \\
& B_{2}(u, v, s, t)=\sum_{u}^{v} \sum_{t}^{s}+\lambda_{t}
\end{aligned}
$$

Fig. 10.6. Diagrammatic representations of $A_{3}(s, u, v), \quad B_{1}(s, t, u, v)$ and $B_{2}(u, v, s, t)$.

and define

$$
\begin{aligned}
A_{3}(s, u, v)= & \tau_{p}(s, v) \tau_{p}(s, u) \tau_{p}(u, v) \\
B_{1}(s, t, u, v)= & \tilde{\tau}_{p}(t, v) \tau_{p}(s, u) \\
B_{2}(u, v, s, t)= & \tau_{p}(u, v) \tau_{p}(u, t) \tau_{p}(v, s) \tau_{p}(s, t) \\
& +\delta_{v, s} \tau_{p}(u, t) \sum_{a \in \mathbb{V}} \tau_{p}(s, a) \tau_{p}(a, u) \tau_{p}(a, t) .
\end{aligned}
$$

The two terms in $B_{2}$ arise from the two events $F^{\prime}$ and $F^{\prime \prime}$ in (10.36). We will write them as $B_{2}^{(1)}$ and $B_{2}^{(2)}$, respectively. The above quantities are represented diagrammatically in Fig. 10.6. In the diagrams, a line joining $a$ and $b$ represents $\tau_{p}(a, b)$. In addition, small bars are used to distinguish a line that represents $\tilde{\tau}_{p}$, in $B_{1}$.

Application of the BK inequality yields

$$
\begin{gathered}
\mathbb{P}_{p}\left(F_{0}\left(x, u_{0}, w_{0}, z_{1}\right)\right) \leq A_{3}\left(x, u_{0}, w_{0}\right) \tau_{p}\left(w_{0}, z_{1}\right), \\
\sum_{v_{N-1}} J\left(u_{N-1}, v_{N-1}\right) \mathbb{P}_{p}\left(F_{N}\left(v_{N-1}, t_{N}, z_{N}, y\right)\right) \\
\leq \frac{B_{1}\left(w_{N-1}, u_{N-1}, z_{N}, t_{N}\right)}{\tau_{p}\left(w_{N-1}, z_{N}\right)} A_{3}\left(y, t_{N}, z_{N}\right),
\end{gathered}
$$




$$
\begin{gathered}
\sum_{v_{i-1}} J\left(u_{i-1}, v_{i-1}\right) \mathbb{P}_{p}\left(F^{\prime}\left(v_{i-1}, t_{i}, z_{i}, u_{i}, w_{i}, z_{i+1}\right)\right) \\
\leq \frac{B_{1}\left(w_{i-1}, u_{i-1}, z_{i}, t_{i}\right)}{\tau_{p}\left(w_{i-1}, z_{i}\right)} B_{2}^{(1)}\left(z_{i}, t_{i}, w_{i}, u_{i}\right) \tau_{p}\left(w_{i}, z_{i+1}\right) \\
\sum_{v_{i-1}, t_{i}} J\left(u_{i-1}, v_{i-1}\right) \mathbb{P}_{p}\left(F^{\prime \prime}\left(v_{i-1}, t_{i}, z_{i}, u_{i}, w_{i}, z_{i+1}\right)\right) \\
\leq \frac{B_{1}\left(w_{i-1}, u_{i-1}, z_{i}, w_{i}\right)}{\tau_{p}\left(w_{i-1}, z_{i}\right)} B_{2}^{(2)}\left(z_{i}, w_{i}, w_{i}, u_{i}\right) \tau_{p}\left(w_{i}, z_{i+1}\right) .
\end{gathered}
$$

Since the second and the third arguments of $B_{2}^{(2)}$ are equal by virtue of the Kronecker delta in (10.47), we can combine (10.50)-(10.51) to obtain

$$
\begin{aligned}
\sum_{v_{i-1}, t_{i}} J( & \left.u_{i-1}, v_{i-1}\right) \mathbb{P}_{p}\left(F\left(v_{i-1}, t_{i}, z_{i}, u_{i}, w_{i}, z_{i+1}\right)\right) \\
& \leq \sum_{t_{i}} \frac{B_{1}\left(w_{i-1}, u_{i-1}, z_{i}, t_{i}\right)}{\tau_{p}\left(w_{i-1}, z_{i}\right)} B_{2}\left(z_{i}, t_{i}, w_{i}, u_{i}\right) \tau_{p}\left(w_{i}, z_{i+1}\right) .
\end{aligned}
$$

Upon substitution of the bounds on the probabilities in (10.48), (10.49) and (10.52) into (10.43), the ratios of two-point functions form a telescoping product that disappears. After relabelling the summation indices, (10.43) becomes

$$
\begin{gathered}
\Pi^{(N)}(x, y) \leq \sum_{\vec{s}, \vec{t}, \vec{u}, \vec{v}} A_{3}\left(x, s_{1}, t_{1}\right) \prod_{i=1}^{N-1}\left[B_{1}\left(s_{i}, t_{i}, u_{i}, v_{i}\right) B_{2}\left(u_{i}, v_{i}, s_{i+1}, t_{i+1}\right)\right] \\
\times B_{1}\left(s_{N}, t_{N}, u_{N}, v_{N}\right) A_{3}\left(u_{N}, v_{N}, y\right)
\end{gathered}
$$

The bound (10.53) is valid for $N \geq 1$, and the summation is over all $s_{1}, \ldots, s_{N}$, $t_{1}, \ldots, t_{N}, u_{1}, \ldots, u_{N}, v_{1}, \ldots, v_{N}$. For $N=1,2$, the right hand side is represented diagrammatically in Fig. 10.7. In the diagrams, unlabelled vertices are summed over $\mathbb{V}$.

(a)

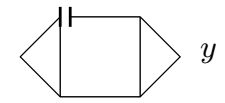

(b)

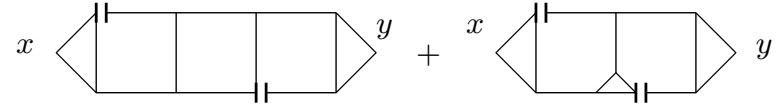

Fig. 10.7. The diagrams bounding (a) $\Pi^{(1)}(x, y)$ and (b) $\Pi^{(2)}(x, y)$. 


\subsection{Diagrammatic Estimates for Percolation}

In this section, we indicate how the Feynman diagrams can be bounded in terms of quantities related to the triangle diagram. We now specialize to the case where the graph $\mathbb{G}$ has vertex set $\mathbb{Z}^{d}$ and edge set given by $y-x \in \Omega$ with either (1.1) or (1.2), and make use of the additive structure and the $x \mapsto-x$ symmetry. We will write $\tau_{p}(y-x)$ in place of $\tau_{p}(x, y), p|\Omega| D(y-x)$ in place of $J(x, y)$, and $\Pi^{(N)}(y-x)$ in place of $\Pi^{(N)}(x, y)$. We recall the definitions of $\nabla_{p}(x)$ and $\tilde{\tau}_{p}(x)$ in (9.51) and (10.44), and define

$$
\begin{gathered}
T_{p}(x)=\left(\tau_{p} * \tau_{p} * \tilde{\tau}_{p}\right)(x), \\
T_{p}=\sup _{x \in \mathbb{Z}^{d}} T_{p}(x), \\
\bar{\nabla}_{p}=\sup _{x \in \mathbb{Z}^{d}} \nabla_{p}(x)=\sup _{x \in \mathbb{Z}^{d}}\left(\tau_{p} * \tau_{p} * \tau_{p}\right)(x) .
\end{gathered}
$$

Theorem 10.2. For $N=0$,

$$
\sum_{x \in \mathbb{Z}^{d}} \Pi^{(0)}(x) \leq T_{p}
$$

For $N \geq 1$

$$
\sum_{x \in \mathbb{Z}^{d}} \Pi^{(N)}(x) \leq \bar{\nabla}_{p}\left(2 T_{p} \bar{\nabla}_{p}\right)^{N} .
$$

The bounds of Theorem 10.2 play the role played by Theorem 4.1 for the self-avoiding walk, although for simplicity we discuss here only bounds on $\sum_{x} \Pi^{(N)}(x)$ and not bounds weighted by $|x|^{2}$ or $[1-\cos (k \cdot x)]$. The latter can be found in [94] or [32], respectively. The theorem can be applied in settings where $2 T_{p} \bar{\nabla}_{p}$ is small, to allow for summation of (10.58) over $N$. For this, Proposition 5.3 is replaced by the estimate

$$
\int_{[-\pi, \pi]^{d}} \frac{\hat{D}(k)^{2}}{[1-\hat{D}(k)]^{3}} \frac{\mathrm{d}^{d} k}{(2 \pi)^{d}} \leq \beta,
$$

with $\beta=K(d-6)^{-1}$ for the nearest-neighbour model, and $\beta=K L^{-d}$ for the spread-out model in dimensions $d>6$ (see [32] for this perspective).

Proof of Theorem 10.2. We first prove (10.57). By (10.7) and the BK inequality,

$$
\Pi^{(0)}(x)=\mathbb{P}(0 \Leftrightarrow x)-\delta_{0, x} \leq \tau_{p}(x)^{2}-\delta_{0, x} .
$$

For $x \neq 0$, the event $\{0 \leftrightarrow x\}$ is the union over neighbors $y$ of the origin of $\{\{0, y\}$ occupied $\} \circ\{y \leftrightarrow x\}$. Thus, by the BK inequality,

$$
\tau_{p}(x) \leq p|\Omega|\left(D * \tau_{p}\right)(x)=\tilde{\tau}_{p}(x) \quad(x \neq 0) .
$$

Therefore, 


$$
\sum_{x \in \mathbb{Z}^{d}} \Pi^{(0)}(x) \leq \sum_{x \in \mathbb{Z}^{d}} \tau_{p}(x) \tilde{\tau}_{p}(x) \leq T_{p}(0) .
$$

This proves (10.57).

Next, we prove (10.58). For $N \geq 1$, let

$$
\begin{aligned}
& \Psi^{(N)}\left(s_{N+1}, t_{N+1}\right) \\
& =\sum_{\vec{s}, \vec{t}, \vec{u}, \vec{v}} A_{3}\left(0, s_{1}, t_{1}\right) \prod_{i=1}^{N}\left[B_{1}\left(s_{i}, t_{i}, u_{i}, v_{i}\right) B_{2}\left(u_{i}, v_{i}, s_{i+1}, t_{i+1}\right)\right],
\end{aligned}
$$

where the sum is over $s_{1}, \ldots, s_{N}, t_{1}, \ldots, t_{N}, u_{1}, \ldots, u_{N}, v_{1}, \ldots, v_{N}$. For convenience, we define $\Psi^{(0)}(x, y)=A_{3}(0, x, y)$, so that for $N \geq 1$,

$$
\Psi^{(N)}(x, y)=\sum_{u_{N}, v_{N}, s_{N}, t_{N}} \Psi^{(N-1)}\left(s_{N}, t_{N}\right) B_{1}\left(s_{N}, t_{N}, u_{N}, v_{N}\right) B_{2}\left(u_{N}, v_{N}, x, y\right) .
$$

Since

$$
\sum_{x} A_{3}\left(u_{N}, v_{N}, x\right) \leq \sum_{x, y} B_{2}\left(u_{N}, v_{N}, x, y\right),
$$

it follows from (10.53) that

$$
\sum_{x} \Pi^{(N)}(x) \leq \sum_{x, y} \Psi^{(N)}(x, y)
$$

and bounds on $\Pi^{(N)}$ can be obtained from bounds on $\Psi^{(N)}$. We prove bounds on $\Psi^{(N)}$, and hence on $\Pi^{(N)}$, by induction on $N$.

The induction hypothesis is that

$$
\sum_{x, y} \Psi^{(N)}(x, y) \leq \bar{\nabla}_{p}\left(2 T_{p} \bar{\nabla}_{p}\right)^{N}
$$

For $N=0,(10.67)$ is true since

$$
\sum_{x, y} A_{3}(0, x, y) \leq \bar{\nabla}_{p}
$$

If we assume (10.67) is valid for $N-1$, then by (10.64),

$$
\begin{aligned}
\sum_{x, y} \Psi^{(N)}(x, y) \leq & \left(\sum_{s_{N}, t_{N}} \Psi^{(N-1)}\left(s_{N}, t_{N}\right)\right) \\
& \times\left(\sup _{s_{N}, t_{N}} \sum_{u_{N}, v_{N}, x, y} B_{1}\left(s_{N}, t_{N}, u_{N}, v_{N}\right) B_{2}\left(u_{N}, v_{N}, x, y\right)\right)
\end{aligned}
$$

and (10.67) then follows once we prove that

$$
\sup _{s, t} \sum_{u, v, x, y} B_{1}(s, t, u, v) B_{2}(u, v, x, y) \leq 2 T_{p} \bar{\nabla}_{p} .
$$


It remains to prove (10.70). There are two terms, due to the two terms in (10.47), and we bound each term separately. The first term is bounded as

$$
\begin{aligned}
\sup _{s, t} & \sum_{u, v, x, y} \tilde{\tau}_{p}(v-t) \tau_{p}(u-s) \tau_{p}(y-u) \tau_{p}(x-v) \tau_{p}(v-u) \tau_{p}(x-y) \\
\quad & \sup _{s, t} \sum_{u, v} \tilde{\tau}_{p}(v-t) \tau_{p}(u-s) \tau_{p}(v-u)\left(\sum_{x, y} \tau_{p}(y-u) \tau_{p}(x-v) \tau_{p}(x-y)\right) \\
\leq & \bar{\nabla}_{p} \sup _{s, t} \sum_{u, v} \tilde{\tau}_{p}(v-t) \tau_{p}(u-s) \tau_{p}(v-u) \\
\quad & T_{p} \bar{\nabla}_{p} .
\end{aligned}
$$

The second term is bounded similarly, making use of symmetry, by

$$
\begin{aligned}
\sup _{s, t} & \sum_{u, v, x, y, a} \tilde{\tau}_{p}(v-t) \tau_{p}(u-s) \delta_{v, x} \tau_{p}(y-u) \tau_{p}(x-a) \tau_{p}(u-a) \tau_{p}(y-a) \\
= & \sup _{s, t} \sum_{a, y, u}\left(\left(\tilde{\tau}_{p} * \tau\right)(a-t) \tau_{p}(u-s)\right)\left(\tau_{p}(y-u) \tau_{p}(u-a) \tau_{p}(y-a)\right) \\
= & \sup _{s, t} \sum_{y^{\prime}, a^{\prime}} T_{p}\left(a^{\prime}+s-t\right) \tau_{p}\left(y^{\prime}\right) \tau_{p}\left(a^{\prime}\right) \tau_{p}\left(y^{\prime}-a^{\prime}\right) \\
\leq & \left(\sup _{a^{\prime}, s, t} T_{p}\left(a^{\prime}+s-t\right)\right)\left(\sum_{y^{\prime}, a^{\prime}} \tau_{p}\left(y^{\prime}\right) \tau_{p}\left(a^{\prime}\right) \tau_{p}\left(y^{\prime}-a^{\prime}\right)\right) \\
\leq & T_{p} \bar{\nabla}_{p}
\end{aligned}
$$

where $a^{\prime}=a-u, y^{\prime}=y-u$. This completes the proof of (10.70) and hence of (10.58).

\section{Results for Percolation}

In this section, we survey results that have been obtained for percolation using the expansion of Section 10, and extensions of this expansion. For the results on $\mathbb{Z}^{d}$ with bonds $\{x, y\}$ specified by $y-x \in \Omega$, we consider the nearestneighbour model with $\Omega$ given by (1.1) and the spread-out model with $\Omega$ given by (1.2).

\subsection{Critical Exponents}

The following infrared bound was proved in [94] (abbreviated versions appeared in $[92,93])$.

Theorem 11.1. There is a constant independent of $p$ and $k$ such that

$$
\hat{\tau}_{p}(k) \leq \mathrm{const}|k|^{-2}
$$


uniformly in $p<p_{c}$ and $k \in[-\pi, \pi]^{d}$, for the nearest-neighbour model in dimensions $d \geq d_{0}$ for some $d_{0} \geq 6$, and for the spread-out model if $d>6$ and $L \geq L_{0}$ for some $L_{0}=L_{0}(d) \gg 1$.

For the spread-out model, much more general models are actually considered in [94]. In particular, models with bonds of unbounded length are allowed if the occupation probability decays sufficiently rapidly with bond length. For the nearest-neighbour model, the constant $d_{0}$ in Theorem 11.1 was found to be $d_{0}=48$ in [94], and this was improved to $d_{0}=19$ in [100], although the lengthy numerical calculations that produce these particular numbers were never published.

The triangle condition follows immediately from the infrared bound. To see this, we first note from (9.51), (9.53) and the monontone convergence theorem ${ }^{10}$ that the infrared bound gives

$$
\begin{aligned}
\mathrm{T}\left(p_{c}\right) & =\lim _{p \rightarrow p_{c}^{-}} \sum_{x, y \in \mathbb{Z}^{d}} \tau_{p}(x) \tau_{p}(y-x) \tau_{p}(y) \\
& =\lim _{p \rightarrow p_{c}^{-}} \int_{[-\pi, \pi]^{d}} \hat{\tau}_{p}(k)^{3} \frac{\mathrm{d}^{d} k}{(2 \pi)^{d}} \\
& \leq \text { const } \int_{[-\pi, \pi]^{d}} \frac{1}{|k|^{6}} \frac{\mathrm{d}^{d} k}{(2 \pi)^{d}},
\end{aligned}
$$

and the integral is finite for $d>6$. In addition, it is shown in [94] that $\overline{\mathrm{T}}\left(p_{c}\right) \leq O\left(d^{-1}\right)$ for the nearest-neighbour model (see [94, Lemma 4.4]), and the results of [94] can be extended in a straightforward way to prove that $\overline{\mathrm{T}}\left(p_{c}\right) \leq O\left(L^{-d}\right)$ for the spread-out model (see [118, Lemma 3.1]). This is important for application of Theorem 10.2.

It follows from the differential inequalities of Sections 9.2-9.3 (see Corollaries 9.3 and 9.8 and Theorems 9.5 and 9.10) that under the hypotheses of Theorem 11.1,

$$
\begin{aligned}
\chi(p) & \simeq\left(p_{c}-p\right)^{-1} & & \text { as } p \rightarrow p_{c}^{-} \\
M\left(p_{c}, \gamma\right) & \simeq \gamma^{1 / 2} & & \text { as } \gamma \rightarrow 0^{+} \\
\theta(p) & \simeq\left(p-p_{c}\right) & & \text { as } p \rightarrow p_{c}^{+} .
\end{aligned}
$$

(As defined in (2.32), the symbol $\simeq$ implies upper and lower bounds with possibly different constants.) This shows that $\gamma=\beta=1$ and $\delta=2$. Also, it was shown in [165] that the triangle condition implies that

$$
\frac{\mathbb{E}_{p}\left(|C(0)|^{m+1}\right)}{\mathbb{E}_{p}\left(|C(0)|^{m}\right)} \simeq\left(p_{c}-p\right)^{-2} \quad \text { as } p \rightarrow p_{c}^{-}, \text {for } m=1,2, \ldots,
$$

so the gap exponent $\Delta$ exists and takes the value $\Delta=2$ under the hypotheses of Theorem 11.1.

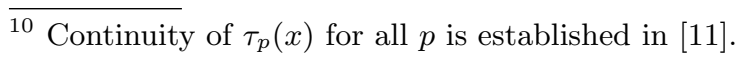


Using a novel argument, it was shown in [89] that

$$
\xi(p) \simeq\left(p_{c}-p\right)^{-1 / 2} \quad \text { as } p \rightarrow p_{c}^{-}
$$

under the hypotheses of Theorem 11.1, which is to say that the critical exponent $\nu$ for the correlation length (9.4) exists and equals $\frac{1}{2}$.

Concerning the critical exponent $\alpha$ for the free energy, less is known. In [210], it is shown that for nearest-neighbour bond percolation on $\mathbb{Z}^{d}$, given any non-negative integer $n$, there exists a dimension $d_{n}$ such that $\kappa(p)$ is $n$ times continuously differentiable for $p \in\left[0, p_{c}\right]$, and hence the derivative $\kappa^{(n)}(p)$ is uniformly bounded on $\left[0, p_{c}\right]$ if $d \geq d_{n}$. The derivatives at $p_{c}$ are interpreted as left derivatives. The possibility is not ruled out in [210] that $\kappa^{(n)}\left(p_{c}^{-}\right)$is equal to zero, although this is not expected to be the case. Comparing with (9.14), the conclusion of [210] is that $\alpha \leq-1$ in high dimensions, consistent with $\alpha=-1$. The methods of [210] apply also to the spread-out model, but the relevance of this is diminished by the fact that the dimension $d_{n}$ grows with $n$, and results for all $d>6$ have not been obtained. For a regular infinite tree of degree $|\Omega| \geq 3$, it is known (see [210]) that the analogue $\kappa_{\text {tree }}(p)$ of $\kappa(p)$ is twice differentiable on $[0,1], \kappa_{\text {tree }}^{\prime \prime \prime}(p)$ has a jump discontinuity at $p=\frac{1}{|\Omega|-1}$, and $\kappa_{\text {tree }}^{(n)}(p)$ is bounded uniformly in $p \neq \frac{1}{|\Omega|-1}$ for each $n$. The exponent $\alpha$ is generally tricky, and remains mysterious even for $d=2$, where other exponents are understood [191].

Concerning the critical exponent $\eta$, recalling the characterization of $\eta$ as $\hat{\tau}_{p_{c}}(k) \sim c|k|^{\eta-2}$, the infrared bound of Theorem 11.1 can be interpreted as a $k$-space statement of the mean-field bound $\eta \geq 0$. This has been greatly extended, in two ways.

Firstly, the following theorem from [91] gives an $x$-space statement that $\eta=0$. Note that it is, in general, not possible to conclude asymptotic behaviour $|x|^{2-d}$ directly from asymptotic behaviour $|k|^{-2}$ of the Fourier transform, without additional regularity of the Fourier transform. A detailed discussion of this point can be found in [158, pp. 32-33]. Thus, additional input beyond Theorem 11.1 is needed to prove the following theorem. The proof of Theorem 11.2 actually proceeds directly in $x$-space, without using Fourier transforms, and is not a corollary of Theorem 11.1.

Theorem 11.2. (a) [90] For $d \geq 19$, there is a constant $a^{\prime}$ depending on $d$, such that the critical two-point function of nearest-neighbour percolation obeys, as $|x| \rightarrow \infty$,

$$
\tau_{p_{c}}(x)=\frac{a^{\prime}}{|x|^{d-2}}\left[1+O\left(\frac{1}{|x|^{2 / d}}\right)\right] .
$$

(b) [91] Fix any $\alpha>0$ (think of $\alpha$ small). For $d>6$, there is a finite constant a depending on $d$ and $L$, and an $L_{0}$ depending on $d$ and $\alpha$, such that for $L \geq L_{0}$ the spread-out model of strictly self-avoiding walk obeys, as $|x| \rightarrow \infty$, 


$$
\tau_{p_{c}}(x)=\frac{a}{|x|^{d-2}}\left[1+O\left(\frac{1}{|x|^{[2 \wedge 2(d-6)]-\alpha}}\right)\right] .
$$

The constant in the error term is uniform in $x$ but may depend on $L$ and $\alpha$; a more careful statement in this regard is given in [91].

Secondly, in $k$-space, the infrared bound has been extended to a joint asymptotic formula as $k \rightarrow 0$ and $\gamma \rightarrow 0^{+}$, at $p=p_{c}$, involving both $\eta=0$ and $\gamma=\frac{1}{2}$. We use translation invariance to rewrite (9.89) as

$$
\tau_{p, \gamma}(x)=\mathbb{P}_{p, \gamma}(0 \leftrightarrow x, 0 \leftrightarrow \mathcal{G}),
$$

and consider the Fourier transform

$$
\hat{\tau}_{p, \gamma}(k)=\sum_{x \in \mathbb{Z}^{d}} \tau_{p, \gamma}(x) \mathrm{e}^{\mathrm{i} k \cdot x}
$$

Theorem 11.3. Let $p=p_{c}, k \in[-\pi, \pi]^{d}, \gamma \in(0,1]$. For nearest-neighbour bond percolation with $d$ sufficiently large, and for spread-out bond percolation with $d>6$ and $L$ sufficiently large, there are functions $\epsilon_{1}(z)$ and $\epsilon_{2}(k)$ with $\lim _{\gamma \rightarrow 0^{+}} \epsilon_{1}(\gamma)=\lim _{k \rightarrow 0} \epsilon_{2}(k)=0$, and constants $C_{2}$ and $D_{2}$ depending on $d$ and $L$, such that

$$
\hat{\tau}_{p_{c}, \gamma}^{(2)}(k)=\frac{C_{2}}{D_{2}^{2}|k|^{2}+2^{3 / 2} \gamma^{1 / 2}}[1+\epsilon(\gamma, k)]
$$

with $|\epsilon(\gamma, k)| \leq \epsilon_{1}(\gamma)+\epsilon_{2}(k)$. In addition, the limit $\hat{\tau}_{p_{c}, 0}(k)=\lim _{\gamma \rightarrow 0^{+}} \hat{\tau}_{p_{c}, \gamma}(k)$ exists and is finite for $k \neq 0$, and obeys

$$
\hat{\tau}_{p_{c}, 0}(k)=\frac{C_{2}}{D_{2}^{2}|k|^{2}}\left[1+\epsilon_{2}(k)\right] .
$$

The factor $2^{3 / 2}$ in (11.12) is present to agree with our convention in Section 16, where (11.12) is related to integrated super-Brownian excursion. By an appeal to universality, Theorem 11.3 strongly suggests that (11.12) and (11.13) should also be valid for the nearest-neighbour model for all $d>6$.

Equation (11.13) is a $k$-space statement that $\eta=0$. There is good reason to be careful with the limit $\gamma \rightarrow 0^{+}$in (11.13). In fact, according to Theorem $11.2, \tau_{p_{c}, 0}(x)$ decays like $|x|^{2-d}$. Therefore it is not summable in $x$, and its Fourier transform is not well-defined without some interpretation. We use the interpretation $\hat{\tau}_{p_{c}, 0}(k)=\lim _{\gamma \rightarrow 0^{+}} \hat{\tau}_{p_{c}, \gamma}(k)$ because $\tau_{p_{c}, 0}(x)$ is then the inverse Fourier transform of $\hat{\tau}_{p_{c}, 0}(k)$. In fact, using monotone convergence in the first step, and using (11.12) and the dominated convergence theorem in the last step, we have

$$
\begin{aligned}
\tau_{p_{c}, 0}(x) & =\lim _{\gamma \rightarrow 0^{+}} \tau_{p_{c}, \gamma}(x) \\
& =\lim _{\gamma \rightarrow 0^{+}} \int_{[-\pi, \pi]^{d}} \hat{\tau}_{p_{c}, \gamma}(k) \mathrm{e}^{-\mathrm{i} k \cdot x} \frac{\mathrm{d}^{d} k}{(2 \pi)^{d}} \\
& =\int_{[-\pi, \pi]^{d}} \hat{\tau}_{p_{c}, 0}(k) \mathrm{e}^{-\mathrm{i} k \cdot x} \frac{\mathrm{d}^{d} k}{(2 \pi)^{d}}
\end{aligned}
$$


Recalling the definition of $\chi(p, \gamma)$ in (9.29), and the representation (9.90), we have $\hat{\tau}_{p_{c}, \gamma}(0)=\chi\left(p_{c}, \gamma\right)$. Setting $k=0$ in (11.12) gives

$$
\chi\left(p_{c}, \gamma\right)=\hat{\tau}_{p_{c}, \gamma}(0)=\gamma^{-1 / 2}\left[2^{-3 / 2} C_{2}+o(1)\right],
$$

as $\gamma \rightarrow 0^{+}$. Integration of this with respect to $\gamma$, using $M\left(p_{c}, 0\right)=0$ and again recalling (9.29), gives

$$
M\left(p_{c}, \gamma\right)=\int_{0}^{\gamma} \frac{1}{1-t} \chi\left(p_{c}, t\right) \mathrm{d} t=\gamma^{1 / 2}\left[2^{-1 / 2} C_{2}+o(1)\right] .
$$

This improves (11.4) to a a statement that $\delta=2$ in the sense of an asymptotic formula.

In [105], a stronger statement that the critical exponent $\delta$ equals 2 is proved for the nearest-neighbour model in sufficiently high dimensions. Namely, it is shown that

$$
\mathbb{P}_{p_{c}}(|C(0)|=n)=\frac{C_{2}}{\sqrt{8 \pi}} \frac{1}{n^{3 / 2}}\left[1+O\left(n^{-\epsilon}\right)\right],
$$

for any fixed $\epsilon<\frac{1}{2}$, if $d$ is large enough. In fact, much more is shown in [105], and the results are summarized below in Section 16.5.

The proofs of Theorem 11.3 and (11.17) are based on an extension of the expansion of Section 10 from an expansion for $\tau_{p}(x)=\tau_{p, 0}(x)$ to an expansion for $\tau_{p, \gamma}(x)$. This new expansion relies heavily on the probabilistic interpretation (9.89) of $\tau_{p, \gamma}(x)$, and is not a minor extension of the expansion of Section 10. Details can be found in [104, 105].

Finally, we mention that it is indicated in [198] that the triangle condition can been extended to continuum percolation [160], if the dimension $d$ is large enough, yielding existence of some critical exponents.

\subsection{The Critical Value}

Several authors have considered the asymptotic behaviour of the critical value $p_{c}(d)$ of bond percolation on $\mathbb{Z}^{d}$, in the limit $d \rightarrow \infty$. Bollobás and Kohayakawa [27], Gordon [82], Kesten [143] and Hara and Slade [94] proved that $p_{c}(d)$ is equal to $\frac{1}{2 d}$ plus an error term of size $O\left((\log d)^{2} d^{-2}\right), O\left(d^{-65 / 64}\right)$, $O\left((\log \log d)^{2}(d \log d)^{-1}\right)$ and $O\left(d^{-2}\right)$, respectively. Recently, Alon, Benjamini and Stacey [17] gave an alternate and relatively short proof that $p_{c}(d)$ is asymptotic to $\frac{1}{2 d}$ as $d \rightarrow \infty$, with an estimate $o\left(d^{-1}\right)$ for the error. The expansion

$$
p_{c}(d)=\frac{1}{2 d}+\frac{1}{(2 d)^{2}}+\frac{7}{2(2 d)^{3}}+\frac{16}{(2 d)^{4}}+\frac{103}{(2 d)^{5}}+\cdots
$$

was reported in [78], but with no rigorous bound on the remainder.

The lace expansion was used in $[101,102]$ to prove that 


$$
p_{c}(d)=\frac{1}{2 d}+\frac{1}{(2 d)^{2}}+\frac{7}{2(2 d)^{3}}+O\left((2 d)^{-4}\right) \text {, }
$$

and a simpler proof of this fact, again using the lace expansion, was given in

[123]. Moreover, it was proved in [122] that there exist rational numbers $a_{i}$ such that $p_{c}(d)$ has an asymptotic expansion

$$
p_{c}(d) \sim \sum_{n=1}^{\infty} \frac{a_{i}}{(2 d)^{i}}
$$

Presumably, this expansion is divergent (though there is no proof of this), and the meaning of (11.20) is that for each $M \geq 1$,

$$
p_{c}(d)=\sum_{i=1}^{M} \frac{a_{i}}{(2 d)^{i}}+O\left(\frac{1}{(2 d)^{M+1}}\right) .
$$

The constant in the error term is permitted to depend on $M$, so (11.21) does not imply convergence.

The basic method of the proofs of (11.19) and (11.20) is similar to that employed to obtain the asymptotic expansion for the connective constant in (2.8), which was discussed around (5.66) and in Exercise 5.18. The starting point is the formula

$$
\chi(p)=\frac{1+\hat{\Pi}_{p}(0)}{1-2 d p\left[1+\hat{\Pi}_{p}(0)\right]}
$$

for the susceptibility, which follows from (10.6) and $\chi(p)=\hat{\tau}_{p}(0)$. The function $\hat{\Pi}_{p}(0)$ is well-behaved up to and including $p=p_{c}$, and the critical point is characterized by the equation

$$
1-2 d p_{c}\left[1+\hat{\Pi}_{p_{c}}(0)\right]=0,
$$

or, equivalently,

$$
p_{c}=\frac{1}{2 d}\left[1+\hat{\Pi}_{p_{c}}(0)\right]^{-1}
$$

This equation can then be studied in a recursive fashion, using estimates on $\hat{\Pi}_{p_{c}}(0)$.

For the spread-out model in dimensions $d>6$, the results of [94] (combined with the above observation from [118] that the triangle is $O\left(L^{-d}\right)$ ) give $p_{c}=$ $|\Omega|^{-1}+O\left(|\Omega|^{-2}\right)$ as $L \rightarrow \infty$. This is improved in [118]. ${ }^{11}$

\subsection{The Incipient Infinite Cluster}

For bond percolation on $\mathbb{Z}^{d}$, it is believed that there is no percolation at the critical point in all dimensions $d \geq 2$. At present, proofs of this fact

\footnotetext{
${ }^{11}$ Note that $p_{c}$ in $[118]$ is $|\Omega|$ times our $p_{c}$.
} 
are restricted to 2-dimensional and high-dimensional models. The notion of the incipient infinite percolation cluster (IIC) is an attempt to describe the infinite structure that is emerging but not yet materialized at the critical point. Various aspects of the IIC are discussed in [5]. There is currently no existence theory for the IIC that is applicable in general dimensions.

For bond percolation on $\mathbb{Z}^{2}$, Kesten [142] constructed the IIC as a measure on bond configurations in which the origin is almost surely connected to infinity. He gave two different constructions, both leading to the same measure. One construction is to condition on the event that the origin is connected to infinity, with $p>p_{c}$, and take the limit $p \rightarrow p_{c}^{+}$. A second construction is to condition on the event that the origin is connected to the boundary of a box of radius $n$, with $p=p_{c}$, and let $n \rightarrow \infty$. More recently, Járai [135, 136] has shown that several other definitions of the IIC on $\mathbb{Z}^{2}$ yield the same measure as Kesten's. These include the inhomogeneous model of [51], and definitions in terms of invasion percolation [49], the largest cluster in a large box [35], and spanning clusters [5]. The incipient infinite cluster is thus a natural and robust object that can be constructed in many different ways, at least in dimension $d=2$.

In [115], van der Hofstad and Járai used the lace expansion to give two constructions of the IIC above the upper critical dimension. To describe these constructions, we need the following definitions.

Let $\mathcal{F}$ denote the $\sigma$-algebra of events. A cylinder event is an event that is determined by the occupation status of a finite set of bonds. We denote the algebra of cylinder events by $\mathcal{F}_{0}$. Then $\mathcal{F}$ is the $\sigma$-algebra generated by $\mathcal{F}_{0}$. For a first definition of the IIC, given $x \in \mathbb{Z}^{d}$, we begin by defining $\mathbb{P}_{x}$ by

$$
\mathbb{P}_{x}(E)=\frac{1}{\tau_{p_{c}}(x)} \mathbb{P}_{p_{c}}(E \cap\{0 \leftrightarrow x\}) \quad\left(E \in \mathcal{F}_{0}\right) .
$$

We then define $\mathbb{P}_{\infty}$ by setting

$$
\mathbb{P}_{\infty}(E)=\lim _{x \rightarrow \infty} \mathbb{P}_{x}(E) \quad\left(E \in \mathcal{F}_{0}\right),
$$

assuming the limit exists. The following theorem shows that, at least in high dimensions, this definition produces a probability measure on $\mathcal{F}$, the IIC measure, under which the origin is almost surely connected to infinity.

Theorem 11.4. Consider nearest-neighbour or spread-out percolation on $\mathbb{Z}^{d}$. For the nearest-neighbour model there is a $d_{0} \gg 6$, and for the spread-out model there is an $L_{0}=L_{0}(d) \gg 1$, such that the following statements hold for $d \geq d_{0}$, and for $d>6$ and $L \geq L_{0}$, respectively. The limit in (11.26) exists for every cylinder event $E \in \mathcal{F}_{0}$, independently of the manner in which $x$ goes to infinity. Moreover, $\mathbb{P}_{\infty}$ extends to a probability measure on the $\sigma$-algebra $\mathcal{F}$, and the origin is almost surely connected to infinity under $\mathbb{P}_{\infty}$.

The proof of Theorem 11.4 exhibits an explicit cancellation between the numerator and denominator in (11.25). The asymptotic behaviour of the denominator is given by Theorem 11.2 as a multiple of $|x|^{2-d}$. For the numerator, 
an expansion is developed which is like the expansion for the two-point function, but with notice taken of the fact that the event $E$ occurs. The event $E$ depends on only finitely many bonds, and hence is a local effect in the limit $|x| \rightarrow \infty$. Details of the method of proof can be found in [115].

In [115], a second construction of the IIC measure $\mathbb{P}_{\infty}$ is also given. This construction uses a limit from the subcritical side, and proceeds as follows. For $p<p_{c}$, let

$$
\mathbb{Q}_{p}(E)=\frac{1}{\chi(p)} \sum_{x \in \mathbb{Z}^{d}} \mathbb{P}_{p}(E \cap\{0 \leftrightarrow x\}) \quad\left(E \in \mathcal{F}_{0}\right),
$$

and

$$
\mathbb{Q}_{p_{c}}(E)=\lim _{p \rightarrow p_{c}^{-}} \mathbb{Q}_{p}(E) \quad\left(E \in \mathcal{F}_{0}\right),
$$

assuming the limit exists. It is a theorem of [115] that the limit does exist, and that $\mathbb{Q}_{p_{c}}=\mathbb{P}_{\infty}$, under the high-dimension hypotheses of Theorem 11.4.

Once the IIC measure $\mathbb{P}_{\infty}$ has been constructed, it is natural to ask for its properties, beyond the fact that the origin is almost surely connected to infinity under $\mathbb{P}_{\infty}$. The following properties are proved in [115] for the spreadout model, and they are expected to be true also for the nearest-neighbour model.

An infinite connected graph is said to have a single end if the complement of any finite subgraph contains exactly one infinite connected component. In particular, an infinite connected graph has a single end if any two infinite selfavoiding paths in the set have infinitely many bonds in common. It is proved in [115] that for the spread-out model in dimensions $d>6$ with $L$ sufficiently large, under $\mathbb{P}_{\infty}$ any two infinite self-avoiding paths in the cluster of the origin almost surely have infinitely many bonds in common, and hence the cluster has a single end. In addition, the IIC two-point function obeys

$$
\mathbb{P}_{\infty}(0 \leftrightarrow y) \simeq \frac{1}{|y|^{d-4}},
$$

whereas

$$
\mathbb{P}_{\infty}(\{0 \leftrightarrow y\} \circ\{y \leftrightarrow \infty\}) \simeq \frac{1}{|y|^{d-2}} .
$$

Intuitively, these two formulas say that the cluster of the origin is 4-dimensional under $\mathbb{P}_{\infty}$, whereas the infinite backbone connecting the origin to infinity is 2-dimensional.

It is plausible that the IIC could also be constructed by taking $p=p_{c}$, conditioning on the event that the origin is in a cluster containing exactly $n$ vertices, and letting $n \rightarrow \infty$. This particular construction has not been carried out, but a closely related issue has been studied in [103, 104, 105], in high dimensions. In these papers, the cluster of the origin is conditioned to have size $n$, the lattice spacing is simultaneously rescaled by $n^{-1 / 4}$, and then the limit $n \rightarrow \infty$ is taken. This limit should be regarded as the scaling limit of 
the IIC. We discuss this issue in more detail in Section 16.5, where the scaling limit of the IIC in high dimensions is related to integrated super-Brownian excursion.

Exercise 11.5. The event $\{0 \leftrightarrow y\}$ is not a cylinder event, but it is proved in [115] that (11.26) nevertheless does hold for $E=\{0 \leftrightarrow y\}$. Assuming this, complete the following outline of proof of the upper bound of (11.29).

(a) Apply (9.84) to conclude that

$$
\frac{1}{\tau_{p_{c}}(x)} \mathbb{P}_{p_{c}}(0 \leftrightarrow y, 0 \leftrightarrow x) \leq \sum_{z \in \mathbb{Z}^{d}} \tau_{p_{c}}(z) \tau_{p_{c}}(y-z) \frac{\tau_{p_{c}}(x-z)}{\tau_{p_{c}}(x)} .
$$

Using Theorem 11.2, show that the contribution to the above sum due to $|x-z| \leq \frac{1}{2}|x|$ vanishes in the limit $|x| \rightarrow \infty$.

(b) Suppose $d>4$. Prove that if $|f(x)| \leq(|x|+1)^{2-d}$ and $|g(x)| \leq(|x|+1)^{2-d}$ then $|(f * g)(x)| \leq$ const $(|x|+1)^{4-d}$. (See [91, Proposition 1.7] for extensions.) (c) Analyze the contribution due to $|x-z|>\frac{1}{2}|x|$ by noting that in this case the ratio of two-point functions in the summation is uniformly bounded and converges to 1 as $|x| \rightarrow \infty$.

\subsection{Percolation on Finite Graphs}

It is natural to ask how the percolation phase transition is modified if the infinite graph $\mathbb{Z}^{d}$ is replaced by a large finite subgraph, such as a $d$-dimensional box of large radius. Such matters are actually essential to interpret properly the results of computer simulations of percolation, which are necessarily performed on a finite graph. Quite generally, one can inquire about the nature of the percolation phase transition on arbitrary finite graphs. The best understood setting for this question is the complete graph $K_{V}$.
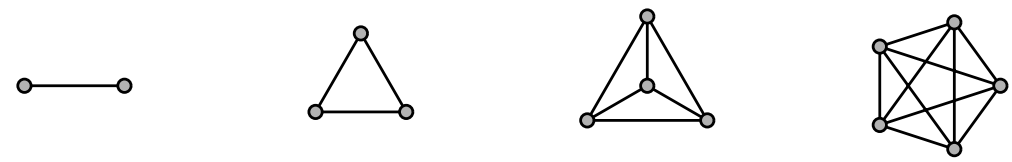

Fig. 11.1. The complete graphs $K_{2}, K_{3}, K_{4}, K_{5}$.

\subsubsection{The Complete Graph}

The complete graph $K_{V}$ is the graph consisting of $V$ vertices, with an edge joining each of the $\left(\begin{array}{c}V \\ 2\end{array}\right)$ pairs of vertices. The percolation model is defined as usual: edges are independently occupied with probability $p$ and vacant with probability $1-p$. In the combinatorial literature, percolation on the complete 
graph is often referred to as random subgraphs of the complete graph, or, more briefly, as the random graph. The substantial theory currently known for the random graph is described in the recent books [26, 134].

For $K_{V}$, the susceptibility $\chi(p)=\mathbb{E}_{p}|C(0)|$ (where 0 denotes an arbitrary vertex) varies continuously between $\chi(0)=1$ and $\chi(p)=V$. In particular, it is of course never infinite. Similarly, the probability that the origin is an infinite cluster is always zero, and thus the phase transition cannot be characterized by the divergence of $\chi$, or the non-vanishing of $\theta$. Nevertheless, there is a phase transition, as suggested by Fig. 11.2. The transition corresponds to an abrupt change in the size $\left|\mathcal{C}_{\max }\right|$ of a cluster $\mathcal{C}_{\max }$ of maximal size, as $p$ is varied through the critical value $p_{c}=\frac{1}{|\Omega|}$, where $|\Omega|=V-1$ is the degree of the complete graph.
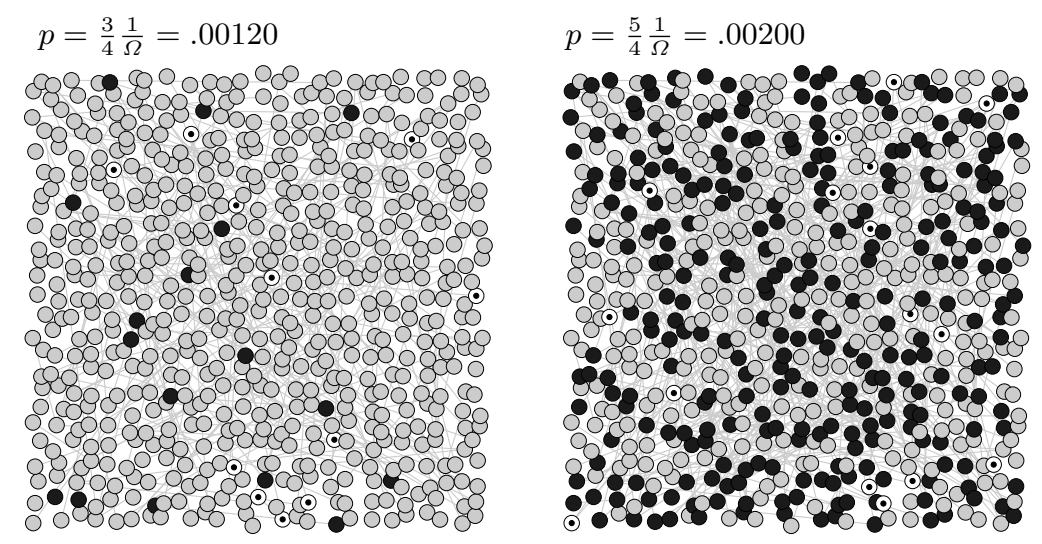

Fig. 11.2. The largest cluster (black) and second largest cluster (dots) in random subgraphs of $K_{625}$. These clusters have size 17 and 11 on the left, and 284 and 16 on the right. The hundreds of edges in the subgraphs are not clearly shown.

The phase transition on $K_{V}$ was first studied by Erdős and Rényi [71]. We will say that a sequence of events $E_{V}$ occurs with high probability, denoted w.h.p., if $\mathbb{P}\left(E_{V}\right) \rightarrow 1$ as $V \rightarrow \infty$. Erdős and Rényi showed that when $p$ is scaled as $(1+\epsilon) V^{-1}$, there is a phase transition at $\epsilon=0$ in the sense that w.h.p.

$$
\left|\mathcal{C}_{\text {max }}\right| \simeq \begin{cases}\log V & \text { if } \epsilon<0, \\ V^{2 / 3} & \text { if } \epsilon=0, \\ V & \text { if } \epsilon>0 .\end{cases}
$$

The bounds of (11.31) are valid for fixed $\epsilon$, independent of $V$.

The results of Erdős and Rényi were substantially extended by Bollobás [25] and Łuczak [156]. In particular, they showed that there is a scaling window of width $V^{-1 / 3}$ in the sense that if $p=\left(1+\Lambda_{V} V^{-1 / 3}\right) V^{-1}$, then w.h.p. 


$$
\left|\mathcal{C}_{\max }\right|\left\{\begin{array}{l}
\ll V^{2 / 3} \text { if } \Lambda_{V} \rightarrow-\infty, \\
\simeq V^{2 / 3} \text { if } \Lambda_{V} \text { is uniformly bounded in } V \\
\gg V^{2 / 3} \text { if } \Lambda_{V} \rightarrow+\infty
\end{array}\right.
$$

Here, we are using the notation $f(V) \ll g(V)$ to mean that $f(V) / g(V) \rightarrow 0$ as $V \rightarrow \infty$, while $f(V) \gg g(V)$ means that $f(V) / g(V) \rightarrow \infty$ as $V \rightarrow \infty$. A great deal more is known, and can be found in $[26,134]$. In particular, above the scaling window, taking $\epsilon>0$ independent of $V$ for simplicity, the second largest cluster has size $\simeq \log V$, much smaller than the largest cluster. This is analogous to the uniqueness of the infinite cluster for supercritical percolation on $\mathbb{Z}^{d}[46]$.

The complete graph is a particularly simple example of a finite graph. It has a high degree of symmetry, and calculations based on counting arguments can go a long way. What if the complete graph is replaced by a finite graph with more geometrical structure, and less symmetry?

\subsubsection{High-Dimensional Transitive Finite Graphs}

A detailed study of the phase transition on high-dimensional finite graphs was initiated in [31] and continued in $[32,33,122,123]$. Let $\mathbb{G}=(\mathbb{V}, \mathbb{B})$ be a graph, where $\mathbb{V}$ is a vertex set of cardinality $V$, and $\mathbb{B}$ is the edge set. A bijective $\operatorname{map} \varphi: \mathbb{V} \rightarrow \mathbb{V}$ is called a graph isomorphism if $\{\varphi(x), \varphi(y)\} \in \mathbb{B}$ whenever $\{x, y\} \in \mathbb{B}$, and $\mathbb{G}$ is called vertex transitive if for every pair of vertices $x, y \in \mathbb{V}$ there is a graph isomorphism $\varphi$ with $\varphi(x)=y$. In a vertex transitive graph, the graph "looks the same" from each vertex. Transitive graphs are by definition regular, i.e., each vertex has the same degree. We denote the degree by $|\Omega|$. Let $\mathbb{G}$ be any finite connected vertex transitive graph.

In [31], the critical point of $\mathbb{G}$ is defined by analogy with the complete graph, for which it is known that the expected cluster size of any particular vertex, for $p$ inside the scaling window, is of order $V^{1 / 3}$. Motivated by this fact, the critical threshold $p_{c}=p_{c}(\mathbb{G}, \lambda)$ of $\mathbb{G}$ was defined to be the unique solution to the equation

$$
\chi\left(p_{c}\right)=\lambda V^{1 / 3},
$$

where $\lambda$ is a positive parameter whose choice is at our disposal. This flexibility in the choice of $\lambda$ is based on the idea is that as long as $\lambda$ varies only weakly with $V$, e.g., if $\lambda$ is a constant, then the value of $p_{c}(\mathbb{G}, \lambda)$ should lie in the critical scaling window. In any case, it is necessary to assume that $1<\lambda V^{1 / 3}<$ $V$, so that $p_{c}$ is well defined and $0<p_{c}<1$. To justify the definition, a theorem that a transition really does take place at $p_{c}$ is required.

The definition (11.33) is appropriate for graphs that obey mean-field behavior, which we expect only for graphs that are in some sense "highdimensional." In particular, it is argued in [31] that (11.33) should be replaced by $\chi\left(p_{c}\right)=\lambda V^{(\delta-1) /(\delta+1)}$, where $\delta$ is the critical exponent for the magnetization (9.18), when $\mathbb{G}$ is a finite periodic approximation to $\mathbb{Z}^{d}$ with $d<6$. Since the mean-field value of $\delta$ is 2 , this is consistent with the definition (11.33). 
In [31], the triangle condition is modified to apply to finite graphs, as follows. Let

$$
\nabla_{p}(x, y)=\sum_{u, v \in V} \tau_{p}(x, u) \tau_{p}(u, v) \tau_{p}(v, y)
$$

denote the (open) triangle diagram. Then the triangle condition is the statement that

$$
\max _{x, y \in \mathbb{V}}\left[\nabla_{p_{c}(\mathbb{G}, \lambda)}(x, y)-\delta_{x, y}\right] \leq a_{0},
$$

where $a_{0}$ is a sufficiently small number. This is a natural analogue of the condition that $\overline{\mathrm{T}}\left(p_{c}\right)$ of $(9.54)$ be small for $\mathbb{Z}^{d}$. Since $\sum_{y} \nabla_{p}(x, y)=\chi(p)^{3}$, the triangle condition implies that $\lambda^{3} \leq V^{-1}+a_{0}$, which requires small $\lambda$.

It is proved in [31] that if the triangle condition holds, then many aspects of the phase transition for $\mathbb{G}$ resemble their counterparts for the complete graph. In particular, $\left|\mathcal{C}_{\max }\right| \simeq V^{2 / 3}$ w.h.p. inside a scaling window of size $V^{-1 / 3}$ around $p_{c}(\mathbb{G}, \lambda)$. Further consequences of the triangle condition are discussed below. The method of proof is based in part on an adaptation of the differential inequality methods of Section 9, and in part on ideas from [35].

To apply the results of [31], it is necessary to establish the triangle condition. This is the subject of [32], where the expansion of Section 10 is used to prove the triangle condition for various high-dimensional graphs. These graphs, denoted $\mathbb{T}_{r, d}$, all have vertex set of the form $\mathbb{V}=\{0,1, \ldots, r-1\}^{d}$, and edge set such that $\{0, x\}$ is an edge if and only if $\{y, y \pm x\}$ is an edge for all vertices $y$ (translation and reflection symmetry). The addition on $\mathbb{V}$ is componentwise addition modulo $r$, corresponding to periodic boundary conditions. It is shown that for such graphs a random walk version of the triangle condition, closely related to (10.59), implies the percolation triangle condition. For many graphs, the random walk triangle condition is relatively easy to establish. Full details can be found in [32]. Among the examples for which the triangle condition is proven in [32] are:

1. the spread-out torus, with $\mathbb{B}=\left\{\{x, y\}: 0<\max _{i=1, \ldots, d}\left|x_{i}-y_{i}\right| \leq L\right\}$, with $d \geq 7$ fixed, $L$ large and fixed, in the limit $r \rightarrow \infty$. Here $V=r^{d}$ and $|\Omega|=(2 L+1)^{d}-1$.

2. the $d$-cube $\mathbb{Q}_{d}$, with $\mathbb{V}=\{0,1\}^{d}$, and with an edge joining distinct vertices that agree in all but one coordinate. Here $V=2^{d}$ and $|\Omega|=d$.

The following is a partial list of results from [32] for a finite connected vertex transitive graph that is assumed to obey the triangle condition. The results include upper and lower bounds below and within a scaling window of width $V^{-1 / 3}$, and upper bounds (but not lower bounds) above the scaling window. For the particular case $\mathbb{G}=\mathbb{Q}_{d}$, lower bounds above the scaling window will be discussed later.

Asymptotic behaviour of the critical value $p_{c}$. This is given by 


$$
p_{c}(\mathbb{G} ; \lambda)=\frac{1}{|\Omega|}\left[1+O\left(|\Omega|^{-1}\right)+O\left(\lambda^{-1} V^{-1 / 3}\right)\right] .
$$

Asymptotics below the window. Let $p=p_{c}-|\Omega|^{-1} \epsilon$ with $\epsilon \geq 0$. If $\epsilon \lambda V^{1 / 3} \rightarrow \infty$ as $V \rightarrow \infty$, then as $V \rightarrow \infty$,

$$
\chi(p)=\frac{1}{\epsilon}\left[1+O\left(|\Omega|^{-1}\right)+O\left(\left(\epsilon \lambda V^{1 / 3}\right)^{-1}\right)\right] .
$$

Comparing with (9.12), this is a statement that $\gamma=1$. In addition, for all $\epsilon \geq 0$

$$
\mathbb{P}_{p}\left(\left|\mathcal{C}_{\max }\right| \leq 2 \chi(p)^{2} \log \left(V / \chi(p)^{3}\right)\right) \geq 1-\frac{\sqrt{e}}{\left[2 \log \left(V / \chi(p)^{3}\right)\right]^{3 / 2}}
$$

In particular, if $\epsilon \lambda V^{1 / 3} \rightarrow \infty$, then $\left|\mathcal{C}_{\max }\right| \leq O\left(\epsilon^{-2} \log V\right)$ w.h.p., and it is also shown in [31] that in this case $\left|\mathcal{C}_{\max }\right| \geq$ const $\epsilon^{-2}$ w.h.p.

Asymptotics inside the window. Fix $\Lambda<\infty$. There exist constants $b_{i}$ such that the following hold for all $p=p_{c}+|\Omega|^{-1} \epsilon$ with $|\epsilon| \leq \Lambda V^{-1 / 3}$, i.e., inside a scaling window of width proportional to $V^{-1 / 3}$. If $k \leq b_{1} V^{2 / 3}$, then

$$
\frac{b_{2}}{\sqrt{k}} \leq \mathbb{P}_{p}(|C(0)| \geq k) \leq \frac{b_{3}}{\sqrt{k}}
$$

The bounds (11.39) are a statement that $\delta=2$ (cf. (9.19)). If $\omega \geq 1$, then

$$
\mathbb{P}_{p}\left(\omega^{-1} V^{2 / 3} \leq\left|\mathcal{C}_{\max }\right| \leq \omega V^{2 / 3}\right) \geq 1-\frac{b_{6}}{\omega}
$$

Finally,

$$
b_{7} V^{1 / 3} \leq \chi(p) \leq b_{8} V^{1 / 3}
$$

In the above statements, the constants $b_{2}$ and $b_{3}$ can be chosen independent of $\lambda$ and $\Lambda$, the constant $b_{8}$ depends on $\Lambda$ and not on $\lambda$, and the constants $b_{1}$, $b_{6}$ and $b_{7}$ depend on both $\lambda$ and $\Lambda$.

Upper bounds above the window. For the supercritical phase, let $p=p_{c}+\epsilon|\Omega|^{-1}$ with $\epsilon \geq 0$. Then for all $\omega>0$,

$$
\mathbb{P}_{p}\left(\left|\mathcal{C}_{\max }\right| \leq \omega\left(V^{2 / 3}+\epsilon V\right)\right) \geq 1-\frac{21}{\omega}
$$

and

$$
\chi(p) \leq 81\left(V^{1 / 3}+\epsilon^{2} V\right) .
$$

Note that $\epsilon V$ dominates $V^{2 / 3}$ and $\epsilon^{2} V$ dominates $V^{1 / 3}$ when $\epsilon \geq \operatorname{const} V^{-1 / 3}$. The bounds (11.42)-(11.43) provide upper bounds on the size of clusters in the supercritical phase. To see that a phase transition occurs at $p_{c}$, one wants a lower bound. The results of [33] provide such a lower bound for the $d$-cube. 


\subsubsection{The $d$-Cube}

For the $d$-cube, it is proved in [33] that there are strictly positive constants $c_{0}, c_{1}, c_{2}$ such that the following holds as $d \rightarrow \infty$ for all $d$-independent $\lambda$ with $0<\lambda \leq c_{0}$ and all $p=p_{c}+\epsilon d^{-1}$ with $\mathrm{e}^{-c_{1} d^{1 / 3}} \leq \epsilon \leq 1$ :

$$
\begin{aligned}
\left|\mathcal{C}_{\max }\right| & \geq c_{2} \epsilon 2^{d} \quad \text { w.h.p. }, \\
\chi(p) & \geq\left(c_{2} \epsilon\right)^{2} 2^{d} .
\end{aligned}
$$

These bounds are complementary to (11.42)-(11.43). This leaves only the tantalizingly small interval $2^{-d / 3} \ll \epsilon \leq \mathrm{e}^{-c_{1} d^{1 / 3}}$ where lower bounds are lacking.

For the $d$-cube, (11.36) states that if $\lambda$ is chosen such that $\lambda^{-1} 2^{-d / 3}=$ $O\left(d^{-1}\right)$, then $p_{c}\left(\mathbb{Q}_{d}, \lambda\right)=\frac{1}{d}+O\left(\frac{1}{d^{2}}\right)$. This result has been extended in [122] to show that there are rational numbers $b_{i}(i \geq 1)$ such that for all positive integers $M$, all $c, c^{\prime}>0$, and all $p$ for which $\chi(p) \in\left[c d^{M}, c^{\prime} d^{-2 M} 2^{n}\right]$,

$$
p=\sum_{i=1}^{M} b_{i} d^{-i}+O\left(d^{-M-1}\right),
$$

where the constant in the error term depends only on $c, c^{\prime}, M$. In particular, the above holds for $p$ such that $\chi(p)=\lambda 2^{d / 3}$ for any fixed $\lambda>0$. Thus

$$
p_{c}\left(\mathbb{Q}_{d}, \lambda\right) \sim \sum_{i=1}^{\infty} b_{i} d^{-i}
$$

is an asymptotic expansion for any fixed $\lambda$ (in fact, the asymptotic expansion is shown in [122] to hold even with substantial $d$-dependence of $\lambda$ ). The asymptotic expansion is conjectured to be divergent. It follows from (11.36) that $b_{1}=1$, and it is shown in [123] that $b_{2}=1$ and $b_{3}=\frac{7}{2}$. Thus,

$$
p_{c}\left(\mathbb{Q}_{d}, \lambda\right)=\frac{1}{d}+\frac{1}{d^{2}}+\frac{7}{2} \frac{1}{d^{3}}+O\left(\frac{1}{d^{4}}\right),
$$

and the first three coefficients in the expansion agree with the first three coefficients in the expansion (11.19) for the critical value for $\mathbb{Z}^{d}$. It is conjectured that the expansions differ in the fourth term, i.e., that $a_{4} \neq b_{4}$.

These results for the $d$-cube extend results of [13], who identified $\frac{1}{d}$ as a threshold by studying the largest cluster for $p=\frac{1}{d}(1+\epsilon)$ with $\epsilon$ independent of $d$, and [28], who obtained results for $\epsilon$ depending on $d$ but not as small as $\frac{1}{d}$.

\subsubsection{Large Finite Boxes in $\mathbb{Z}^{d}$}

For percolation in a box of radius $r$ in $\mathbb{Z}^{d}$, a study of the phase transition was undertaken in [35]. The analysis of [35] is based on certain scaling and 
hyperscaling hypotheses which are conjectured to be valid in dimensions $d=$ $3,4,5,6$ and are known to hold in dimension 2. It provides a partial description of finite-size scaling for percolation below the upper critical dimension. In dimensions $d>6$, hyperscaling does not hold, and a different theory is needed.

In [5], Aizenman considered the size of the largest cluster inside a box of radius $r$ in $\mathbb{Z}^{d}$ for $d>6$, with the bulk boundary condition. Under the bulk boundary condition, vertices inside the box are considered to be connected if there is an occupied path that joins them in the infinite lattice $\mathbb{Z}^{d}$. In particular, connections are permitted to exit the box. It was shown in [5] that for $d>6$, roughly speaking, at $p_{c}$ the largest cluster in the box has size $r^{4}$ and there are of the order of $r^{d-6}$ clusters of this size, under the assumption that $\tau_{p_{c}}(x) \simeq|x|^{2-d}$. This last hypothesis is supplied by Theorem 11.2, under the usual high- $d$ assumptions. The fact that the largest cluster has size $r^{4}$ is another indication of the 4-dimensional character of the IIC, when $d>6$.

In terms of the volume $V=r^{d}$ of the box, the largest cluster at the critical value $p_{c}(d)$ for $\mathbb{Z}^{d}$, under the bulk boundary condition, thus has size of order $r^{4}=V^{4 / d}$, for $d>6$. The question was raised in [5] whether this would change to $r^{2 d / 3}=V^{2 / 3}$ if the periodic boundary condition is used instead of the bulk boundary condition. According to (11.40), for the spreadout model in dimensions $d>6$ with periodic boundary conditions, if $p$ is within a scaling window centred at $p_{c}\left(\mathbb{T}_{r, d} ; \lambda\right)$ (with $\lambda$ small and constant) and of width proportional to $V^{-1 / 3}$, then the largest cluster is of size $V^{2 / 3}$. As was pointed out in [32], an affirmative answer to the question would follow if it could be proved that the critical value $p_{c}(d)$ of the infinite lattice $\mathbb{Z}^{d}$ is within this scaling window. It is an open problem to do so.

\section{Oriented Percolation}

Oriented percolation is a percolation model in which bonds and connections are directed. The model enjoys a Markov property not present in ordinary percolation, and this simplifies some aspects of the problem. In this section, we survey some of the results that have been obtained for the critical behaviour of oriented percolation, above its upper critical dimension $d=4$. The expansions used to obtain these results will be discussed in Section 13.

\subsection{The Phase Transition}

Let $\Omega$ denote either the nearest-neighbour or spread-out sets defined in (1.1)(1.2). Let $\mathbb{Z}^{d} \times \mathbb{Z}_{+}$denote the graph with vertex set consisting of pairs $(x, n)$ with $x \in \mathbb{Z}^{d}$ and $n$ a non-negative integer, and with directed edges given by ordered pairs $((x, n),(y, n+1))$ of vertices whose time variables differ by 1 and whose space variables obey $y-x \in \Omega$. As usual, we refer to edges as bonds. Bonds are independently occupied with probability $p$ and vacant with probability $1-p$. We write $\{(x, m) \rightarrow(y, n)\}$ to denote the event that there is 
a directed path from $(x, m)$ to $(y, n)$ consisting of occupied bonds, i.e., there is a sequence of occupied bonds $\left(\left(u_{i-1}, i-1\right),\left(u_{i}, i\right)\right)$, for $i=m+1, \ldots, n$, such that $\left(u_{m}, m\right)=(x, m)$ and $\left(u_{n}, n\right)=(y, n)$.

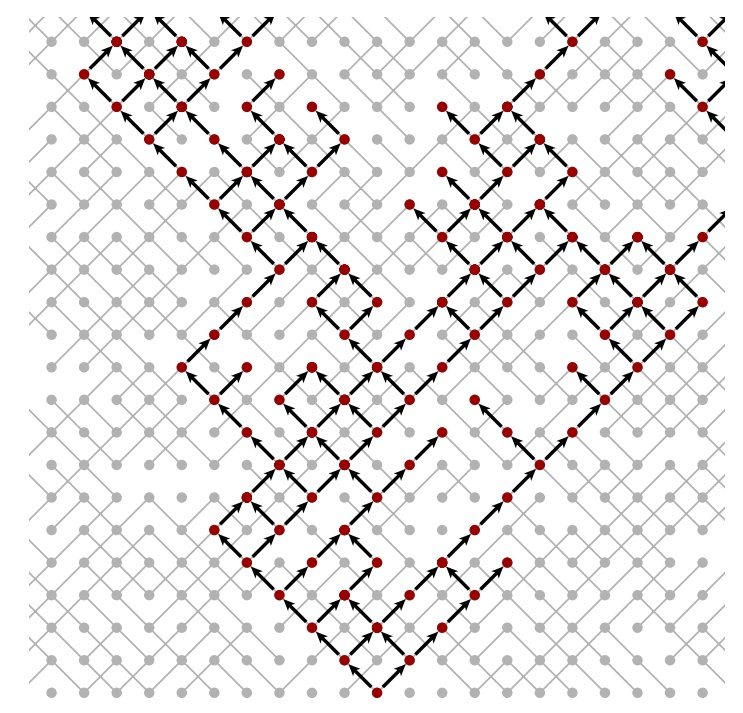

Fig. 12.1. The connected cluster $C(0,0)$ of the origin for oriented percolation on $\mathbb{Z} \times \mathbb{Z}_{+}$. Here $p=0.7$ is a little above $p_{c} \approx 0.645$. For the nearest-neighbour model depicted, the lattice decomposes into two noncommunicating lattices. This will not be the case for the spread-out model when $L \geq 2$.

Let

$$
C(0,0)=\{(x, n):(0,0) \rightarrow(x, n)\}
$$

(see Fig. 12.1). The susceptibility is defined by

$$
\chi(p)=\mathbb{E}_{p}|C(0,0)|
$$

and the percolation probability is defined by

$$
\theta(p)=\mathbb{P}_{p}(|C(0,0)|=\infty) .
$$

The phase transition for oriented percolation occurs at a point that is characterized exactly as it is for ordinary percolation, namely $[7,161,162]$

$$
p_{c}=\sup \{p: \theta(p)=0\}=\sup \{p: \chi(p)<\infty\} .
$$

For oriented percolation it has been proved that $\theta\left(p_{c}\right)=0$ for all dimensions $d \geq 1[22]$. 
The magnetization is defined, for $\gamma \in[0,1]$, by $^{12}$

$$
M(p, \gamma)=1-\sum_{n=1}^{\infty}(1-\gamma)^{n} \mathbb{P}_{p}(|C(0,0)|=n)
$$

Critical exponents analogous to those in Section 9.1 can also be defined for oriented percolation. In particular, writing $c$ to denote a positive constant whose value is unimportant and may change from line to line, the following power laws are believed to hold:

$$
\begin{aligned}
\chi(p) & \sim c\left(p_{c}-p\right)^{-\gamma} \quad \text { as } p \rightarrow p_{c}^{-}, \\
M\left(p_{c}, \gamma\right) & \sim c \gamma^{1 / \delta} \quad \text { as } \gamma \rightarrow 0, \\
\theta(p) & \sim c\left(p-p_{c}\right)^{\beta} \quad \text { as } p \rightarrow p_{c}^{+}, \\
\frac{\mathbb{E}_{p}\left(|C(0,0)|^{m+1}\right)}{\mathbb{E}_{p}\left(|C(0,0)|^{m}\right)} & \sim c\left(p_{c}-p\right)^{-\Delta} \quad \text { as } p \rightarrow p_{c}^{-}, \text {for } m=1,2, \ldots,
\end{aligned}
$$

for some amplitudes $c$ and universal critical exponents $\gamma, \delta, \beta, \Delta$.

It was first predicted in [172] that the upper critical dimension for percolation is $d=4$, or, to emphasize the additional time dimension, $d+1=4+1$. This is now a theorem, as the hyperscaling inequalities of [180] show that the upper critical dimension is not less than $4+1$, and the results below show it is not greater than $4+1$.

\subsection{The Infrared Bound and the Triangle Condition}

The infrared bound and the triangle condition play important roles for the critical behaviour of oriented percolation, just as they do for ordinary percolation. In this section, we discuss this.

The two-point function $\tau_{p, n}(x)$ is defined by

$$
\tau_{p, n}(x)=\mathbb{P}_{p}((0,0) \rightarrow(x, n)) .
$$

When $p$ is understood from the context, we often drop the subscript $p$, writing simply $\tau_{n}(x)$.

By definition,

$$
\hat{\tau}_{p, n}(0)=\sum_{x} \tau_{p, n}(x)=\mathbb{E}_{p}\left|C(0,0) \cap\left(\mathbb{Z}^{d} \times\{n\}\right)\right| .
$$

Thus $\hat{\tau}_{p, n}(0)$ is equal to the expected number of vertices that the origin is connected to at time $n$. This quantity is submuliplicative, in the sense that

$$
\hat{\tau}_{p, m+n}(0) \leq \hat{\tau}_{p, m}(0) \hat{\tau}_{p, n}(0) .
$$

$\overline{12}$ This $\gamma$ should not be confused with the critical exponent in (12.6) which is denoted by the same Greek letter. 
To see this, note that a connection from the origin to $(x, m+n)$ implies the existence of a vertex $(y, m)$ such that $(0,0) \rightarrow(y, m)$ and $(y, m) \rightarrow(x, m+n)$. These two events are independent - this is the Markov property of oriented percolation-so

$$
\tau_{p, m+n}(x) \leq \sum_{y} \tau_{p, m}(y) \tau_{p, n}(x-y),
$$

and the desired inequality follows by summation over $x$. It then follows from (12.12) that the limit

$$
\log r(p)=-\lim _{n \rightarrow \infty} \frac{1}{n} \log \hat{\tau}_{p, n}(0)
$$

exists. By the monotonicity of $\hat{\tau}_{p, n}(0)$ in $p, r(p)$ is non-increasing in $p$. Various arguments lead to the conclusion that $r(p)$ is finite and positive for $p \leq p_{c}$, with $r\left(p_{c}\right)=1$ (see $\left.[167,12]\right)$.

We define a generating function

$$
\hat{t}_{p}(k, z)=\sum_{n=0}^{\infty} \hat{\tau}_{p, n}(k) z^{n}
$$

which has radius of convergence $r(p)$ when $k=0$, by definition of $r(p)$. This is a Fourier-Laplace transform of the two-point function. The infrared bound is an upper bound on $\left|\hat{t}_{p}(k, z)\right|$, given in the following theorem of Nguyen and Yang [167]. The form of the infrared bound can be predicted by comparison with the random walk analogue of (12.15), which is

$$
\sum_{n=0}^{\infty} \hat{D}(k)^{n} z^{n}=\frac{1}{1-z \hat{D}(k)}=\frac{1}{[1-\hat{D}(k)]+(1-z) \hat{D}(k)}
$$

with $D$ given by (1.10). The first term in the denominator is quadratic in $k$, for small $k$, while the second is linear in $1-z$. If we take $z=\mathrm{e}^{\mathrm{i} \theta}$ with $\theta \in[-\pi, \pi]$, an upper bound on the absolute value of (12.16) is const $\left[|k|^{2}+|\theta|\right]^{-1}$.

Theorem 12.1. There exist $d_{0}$ and $L_{0}$ such that the following statement holds for the nearest-neighbour model in dimensions $d \geq d_{0}$ and for the spread-out model in dimensions $d>4$ for $L \geq L_{0}$. Let $p \in\left(0, p_{c}\right], k \in[-\pi, \pi]^{d}, z \in \mathbb{C}$ with $|z|<r(p)$. Then

$$
\left|\hat{t}_{p}(k, z)\right| \leq \frac{C_{1}}{|k|^{2}+|r(p)-z|},
$$

where $C_{1}$ is a constant that may depend on $d$ and $L$.

In particular, for $p<p_{c}$ and $z=\mathrm{e}^{\mathrm{i} \theta}$, the denominator of the infrared bound can be replaced by $|k|^{2}+|\theta|$ in an upper bound. The linear term $|\theta|$, which is a symptom of the orientation in the direction of increasing time, will have an impact on the upper critical dimension. 
The proof of Theorem 12.1 uses the lace expansion discussed below in Section 13.2, with a convergence proof based on the bootstrap Lemma 5.9. An important consequence is an oriented percolation version of the triangle condition, which we discuss next.

The derivation of differential inequalities involving the triangle condition was given in [20] in a very general setting that allows for oriented bonds, and the results of Section 9 have counterparts also for oriented percolation. In brief, an appropriate statement of the triangle condition implies existence of the critical exponents $\gamma, \delta, \beta, \Delta$, in the sense of $\simeq$, with the mean-field values $\gamma=\beta=1$ and $\delta=\Delta=2$. The form of the triangle condition is modified by the orientation, as follows.

Let

$$
\nabla_{p}(x, n)=\sum_{(y, l)} \sum_{(w, m)} \tau_{p, l}(y) \tau_{p, m-l}(w-y) \tau_{p, m-n}(w-x) .
$$

Using $|(x, n)|$ to denote the Euclidean norm of $(x, n)$ in $\mathbb{Z}^{d+1}$, the triangle condition is the statement that

$$
\lim _{R \rightarrow \infty} \sup \left\{\nabla_{p_{c}}(x, n):|(x, n)| \geq R\right\}=0 .
$$

Exercise 12.2. Complete the following outline to prove that the triangle condition follows from (12.17), when $d>4$. Consider $p<p_{c}$ and take $z=\mathrm{e}^{\mathrm{i} \theta}$. Show that (12.18) can be rewritten as

$$
\nabla_{p}(x, n)=\int_{-\pi}^{\pi} \frac{\mathrm{d} \theta}{2 \pi} \int_{[-\pi, \pi]^{d}} \frac{\mathrm{d}^{d} k}{(2 \pi)^{d}} \hat{t}_{p}\left(k, \mathrm{e}^{\mathrm{i} \theta}\right)^{2} \hat{t}_{p}\left(k, \mathrm{e}^{-\mathrm{i} \theta}\right) \mathrm{e}^{-\mathrm{i} k \cdot x} \mathrm{e}^{-\mathrm{i} \theta n} .
$$

The infrared bound implies that

$$
\left|\hat{t}_{p}\left(k, \mathrm{e}^{ \pm \mathrm{i} \theta}\right)\right| \leq \frac{c}{|k|^{2}+|\theta|} .
$$

Conclude from the Riemann-Lebesgue lemma that the triangle condition holds if $d>4$.

In [167], Nguyen and Yang used the generating function methods of [97] to extend their results to obtain certain statements in terms of asymptotic formulas, instead of upper and lower bounds with different constants. For the important particular case $p=p_{c}$, and for the spread-out model, the following theorem from [121] extends the results of [167] (for results when $p<p_{c}$, see [167]). The theorem is proved using the general inductive approach to the lace expansion [120]. Versions of parts (a) and (b) of the theorem are proved in [167], with weaker error estimates, and also for the nearest-neighbour model for $d \geq d_{0}$.

Theorem 12.3. Consider the spread-out model of oriented percolation. Let $d>4, p=p_{c}$, and $\delta \in\left(0,1 \wedge \frac{d-4}{2}\right)$. There is an $L_{0}=L_{0}(d)$ such that 
for $L \geq L_{0}$ there exist positive constants $v$ and $A$ (depending on $d$ and $L$ ), and $C_{1}, C_{2}$ (depending only on $d$ ), such that the following statements hold as $n \rightarrow \infty$ :

(a)

$$
\hat{\tau}_{p_{c}, n}(k / \sqrt{v n})=A \mathrm{e}^{-|k|^{2} / 2 d}\left[1+O\left(|k|^{2} n^{-\delta}\right)+O\left(n^{-(d-4) / 2}\right)\right],
$$

$$
\frac{1}{\hat{\tau}_{p_{c}, n}(0)} \sum_{x}|x|^{2} \tau_{p_{c}, n}(x)=v n\left[1+O\left(n^{-\delta}\right)\right]
$$

$$
C_{1} L^{-d} n^{-d / 2} \leq \sup _{x \in \mathbb{Z}^{d}} \tau_{p_{c}, n}(x) \leq C_{2} L^{-d} n^{-d / 2},
$$

with the error estimate in (a) uniform in $k \in \mathbb{R}^{d}$ with $|k|^{2}(\log n)^{-1}$ sufficiently small.

Theorem 12.3 is the $r=2$ case of a family of results which give the asymptotics of the critical oriented percolation $r$-point functions for all $r \geq 2$. The $r$-point functions for $r \geq 3$ will be discussed in Section 17.3.

Theorem 12.3(a) shows that the expected number of particles $\hat{\tau}_{n}(0)$ to which the origin is connected at time $n$ converges to a nonzero finite constant $A$ as $n \rightarrow \infty$, when $p=p_{c}$. In contrast, for $p<p_{c}$ in general dimensions, $\hat{\tau}_{n}(0)$ decays exponentially to zero, while for $p>p_{c}$ the limit is infinite. (See $[22,86]$ for the relevant shape theorem when $p>p_{c}$.) $n^{1 / 2}$.

Theorem 12.3(b) shows that at $p_{c}$ the length scale at time $n$ is of order

The bounds of Theorem 12.3(c) are consistent with a local central limit theorem. A version of the local central limit theorem similar to Theorem 6.5 applies also to oriented percolation [120, 121].

An extension of Theorem 12.3 to dimensions $d \leq 4$ is discussed below Theorem 14.3, for the case of an oriented percolation model whose range $L$ is unbounded.

Exercise 12.4. Complete the following outline to derive the triangle condition from Theorem 12.3(a, c).

(a) Argue that if $|(x, n)| \geq R$ in (12.18), then the summation index $m$ can be taken to be at least a multiple of $R$ in the sum in (12.18). Thus it suffices to prove that

$$
\lim _{R \rightarrow \infty} \sum_{(y, l)} \sum_{(w, m): m \geq c R} \tau_{p_{c}, l}(y) \tau_{p_{c}, m-l}(w-y) \tau_{p_{c}, m-n}(w-x)=0 .
$$

(b) Obtain this sufficient condition by considering separately the contributions to the sum due to $l \leq m / 2$ and $l>m / 2$. Use $\left\|\tau_{p_{c}, n}\right\|_{1} \leq O(1)$ and $\left\|\tau_{p_{c}, n}\right\|_{\infty} \leq$ $O\left(n^{-d / 2}\right)$, noting that the latter is most effectively applied when $n$ is large. 


\subsection{The Critical Value}

It was observed in [167] that the estimates of [166] imply that the critical value for nearest-neighbour oriented percolation is given asymptotically as

$$
p_{c}=\frac{1}{2 d}+O\left(\frac{1}{(2 d)^{2}}\right) .
$$

Other methods [57] have produced the stronger result that

$$
d^{-1}+\frac{1}{2} d^{-3}+o\left(d^{-3}\right) \leq p_{c} \leq d^{-1}+d^{-3}+O\left(d^{-4}\right),
$$

for a version of oriented percolation in which the forward degree of a vertex is $d$ rather than $2 d$. For the spread-out model, it is shown in [121] that $p_{c}=$ $|\Omega|^{-1}+O\left(|\Omega|^{-2}\right)$ when $d>4$, and this is improved in [118]. ${ }^{13}$

\subsection{The Incipient Infinite Cluster}

The incipient infinite cluster for ordinary percolation was discussed in Section 11.3. In this section, we state existence results for the IIC for spread-out oriented percolation in dimensions $d>4$, and indicate some properties of the IIC.

We write $\mathcal{F}$ for the $\sigma$-algebra of events, and denote the algebra of cylinder events (those determined by the occupation status of a finite set of bonds) by $\mathcal{F}_{0}$. Then $\mathcal{F}$ is the $\sigma$-algebra generated by $\mathcal{F}_{0}$. The following are four possible definitions of the IIC. In each definition, we assume initially that $E \in \mathcal{F}_{0}$.

1. Define $\mathbb{P}_{n}$ by

$$
\mathbb{P}_{n}(E)=\frac{1}{\hat{\tau}_{n}(0)} \sum_{x \in \mathbb{Z}^{d}} \mathbb{P}(E \cap\{(0,0) \rightarrow(x, n)\}),
$$

and, assuming the limit exists, define $\mathbb{P}_{\infty}$ by setting

$$
\mathbb{P}_{\infty}(E)=\lim _{n \rightarrow \infty} \mathbb{P}_{n}(E)
$$

2. Let $S_{n}=\left\{\exists x \in \mathbb{Z}^{d}\right.$ such that $\left.(0,0) \rightarrow(x, n)\right\}$ denote the event that the cluster of the origin survives to time $n$. Define $\mathbb{Q}_{n}$ by

$$
\mathbb{Q}_{n}(E)=\mathbb{P}\left(E \mid S_{n}\right),
$$

and, assuming the limit exists, define $\mathbb{Q}_{\infty}$ by setting

$$
\mathbb{Q}_{\infty}(E)=\lim _{n \rightarrow \infty} \mathbb{Q}_{n}(E) .
$$

$\overline{13 \text { Note that }} p_{c}$ in $[118]$ is $|\Omega|$ times our $p_{c}$. 
3. Fix any $x \in \mathbb{Z}^{d}$, define $\mathbb{P}_{n}^{(x)}$ by

$$
\mathbb{P}_{n}^{(x)}(E)=\mathbb{P}(E \mid\{(0,0) \rightarrow(x, n)\}),
$$

and, assuming the limit exists, define $\mathbb{P}_{\infty}^{(x)}$ by

$$
\mathbb{P}_{\infty}^{(x)}(E)=\lim _{n \rightarrow \infty} \mathbb{P}_{n}^{(x)}(E) .
$$

4. For $p<p_{c}$, let

$$
\mathbb{K}_{p}(E)=\frac{1}{\chi(p)} \sum_{x \in \mathbb{Z}^{d}} \sum_{n=0}^{\infty} \mathbb{P}_{p}(E \cap\{(0,0) \rightarrow(x, n)\}),
$$

and, assuming the limit exists, define $\mathbb{K}_{\infty}$ by

$$
\mathbb{K}_{\infty}(E)=\lim _{p \rightarrow p_{c}^{-}} \mathbb{K}_{p}(E) .
$$

It is natural to conjecture that the above limits exist in all dimensions, and that, moreover, for all $x$,

$$
\mathbb{P}_{\infty}=\mathbb{Q}_{\infty}=\mathbb{P}_{\infty}^{(x)}=\mathbb{K}_{\infty}
$$

The following theorem shows that for sufficiently spread-out oriented percolation in dimensions $d>4$, the limits $\mathbb{P}_{\infty}$ and $\mathbb{K}_{\infty}$ exist and are equal. In addition, $\mathbb{Q}_{\infty}$ exists and equals $\mathbb{P}_{\infty}=\mathbb{K}_{\infty}$ provided we also assume that the critical survival probability

$$
\theta_{n}=\mathbb{P}\left(S_{n}\right)
$$

obeys

$$
\theta_{n} \sim \frac{1}{B n}
$$

for some positive constant $B$. It is a classical fact that the probability that a critical branching process survives for at least $n$ generations is asymptotically a constant multiple of $n^{-1}$, and (12.38) assumes that this mean-field behaviour applies also to oriented percolation when $d>4$. Although there is currently no proof of (12.38), an attempt to prove it is in progress [111, 112] using a point-to-plane expansion rather than the usual point-to-point expansion. The theorem does not make any statement about the limit $\mathbb{P}_{\infty}^{(x)}$.

Theorem 12.5. Let $d+1>4+1$ and $p=p_{c}$. There is an $L_{0}=L_{0}(d)$ such that for $L \geq L_{0}$, the limits $\mathbb{P}_{\infty}$ and $\mathbb{K}_{\infty}$ exist and are equal for every cylinder event $E \in \mathcal{F}_{0}$. If we also assume (12.38), then the limit $\mathbb{Q}_{\infty}$ exists and equals $\mathbb{P}_{\infty}=\mathbb{K}_{\infty}$. Moreover, the limit $\mathbb{P}_{\infty}$ (and hence also $\mathbb{Q}_{\infty}$ and $\mathbb{K}_{\infty}$ ) extends to a probability measure on the $\sigma$-algebra $\mathcal{F}$, and the origin is almost surely connected to infinity under $\mathbb{P}_{\infty}$. 
The proof of Theorem 12.5 can be found in [114], except for the statement concerning $\mathbb{K}_{\infty}$, which is proved in [115]. The proof is based on an extension of the expansion methods discussed in Section 13.

Next, we discuss some properties of the IIC measure $\mathbb{P}_{\infty}$, which are proved in [114]. The Hausdorff dimension of the connected cluster of the origin under the IIC is predicted to equal 4 almost surely, for $d+1>4+1$. The following theorem provides a weaker statement indicating a 4-dimensional aspect to the IIC.

Theorem 12.6. Let $d+1>4+1$ and $p=p_{c}$. There exists $L_{0}=L_{0}(d)$ such that for $L \geq L_{0}$,

$$
\mathbb{E}_{\infty}[|\{(y, m) \in C(0,0):|y| \leq R\}|] \simeq R^{4},
$$

where the expectation is with respect to the IIC measure $\mathbb{P}_{\infty}$, and the absolute value signs represent cardinality of the set.

Let

$$
N_{m}=\left|\left\{y \in \mathbb{Z}^{d}:(0,0) \leftrightarrow(y, m)\right\}\right|
$$

denote the number of vertices at time $m$ to which the origin is connected. The following theorems give the limiting distribution of $N_{m}$ under $\mathbb{P}_{\infty}$ and $\mathbb{Q}_{m}$. The constants $A$ and $B$ in the theorems are the constants of Theorem 12.3(a) and (12.38), while the constant $V$ is the vertex factor which appears in the scaling limit of the 3-point function (see Theorem 17.5 below). Also, we recall that a size-biased exponential random variable with parameter $\lambda$ has density

$$
f(x)=\lambda^{2} x \mathrm{e}^{-\lambda x} \quad(x \geq 0) .
$$

Theorem 12.7. (a) Let $d+1>4+1$ and $p=p_{c}$. There is an $L_{0}=L_{0}(d)$ such that for $L \geq L_{0}$ and for $l=1,2, \ldots$,

$$
\lim _{m \rightarrow \infty} \mathbb{E}_{\infty}\left[\left(\frac{N_{m}}{m}\right)^{l}\right]=\left(\frac{A^{2} V}{2}\right)^{l}(l+1) ! .
$$

Consequently, under $\mathbb{P}_{\infty}, m^{-1} N_{m}$ converges weakly to a size-biased exponential random variable with parameter $\lambda=2 /\left(A^{2} V\right)$.

(b) Assume in addition that (12.38) holds. Then $B=A V / 2$, and for $l=$ $1,2, \ldots$,

$$
\lim _{m \rightarrow \infty} \mathbb{E}_{\mathbb{Q}_{m}}\left[\left(\frac{N_{m}}{m}\right)^{l}\right]=\left(\frac{A^{2} V}{2}\right)^{l} l ! .
$$

Consequently, under $\mathbb{Q}_{m}, m^{-1} N_{m}$ converges weakly to an exponential random variable with parameter $\lambda=2 /\left(A^{2} V\right)$.

Exercise 12.8. Show that (12.42) and (12.43) imply the weak convergence statements made in Theorem 12.7. 
Under the assumption that (12.38) holds, it follows from Theorems 12.7 that $m^{-1} N_{m}$ converges to a size-biased exponential random variable under $\mathbb{Q}_{\infty}=\mathbb{P}_{\infty}$, and to an exponential random variable under $\mathbb{Q}_{m}$. This is similar to the situation for critical branching processes, for which the size-biased exponential distribution occurs when the branching random walk is conditioned to survive to infinite time, and the exponential distribution occurs when the branching random walk is conditioned to survive until time $m$.

The IIC $r$-point functions and the scaling limit of the IIC will be discussed in Section 17.3. Exercise 17.6 concerns the proof of Theorem 12.7(a).

\section{Expansions for Oriented Percolation}

The expansion of Section 10.1 applies directly to oriented percolation, and is sometimes the expansion of choice for oriented percolation [121]. The translation of the expansion of Section 10.1 to the oriented setting is described in Section 13.3.

However, it is also possible to exploit the Markov property of oriented percolation to give alternate, simpler versions of the expansion. A derivation using laces and resummation, first carried out by Nguyen and Yang [166, 208], is described in Section 13.2. Later, a particularly simple derivation of the expansion, based on inclusion-exclusion, was given by Sakai [179]. Sakai's method is described in Section 13.1.

Each expansion gives rise to a recursion equation

$$
\tau_{n}(x)=\left(p|\Omega| D * \tau_{n-1}\right)(x)+\sum_{m=2}^{n-1}\left(\pi_{m} * p|\Omega| D * \tau_{n-1-m}\right)(x)+\pi_{n}(x),
$$

valid for $n \geq 1$. The empty sum is zero for $n \leq 2$, and substituting $n=1$ shows that $\pi_{1}(x)=0$. The function $D$ is given by (1.10). The identity (13.1) can be used as a recursive definition of $\pi_{m}(x)$, by isolating the term $\pi_{n}(x)$ on the right hand side and noting that this expresses it in terms of the two-point function and $\pi_{m}$ with $m<n$. Therefore, $\pi_{n}(x)$ is uniquely determined by (13.1). It follows that all methods for deriving (13.1) must yield exactly the same quantities $\pi_{m}(x)$.

On the other hand, there are differences between the representations for $\pi_{m}(x)$ that are produced by the different expansion methods. Each method gives a representation of $\pi$ as an alternating sum of terms $\pi^{(N)}$. For the percolation expansion of Section 10.1 applied to oriented percolation, the individual terms $\pi^{(N)}$ in the sum are again given by nested expansions as in (10.28). On the other hand, the other two expansions each produce identical representations for $\pi^{(N)}$ by a single expectation, which is conceptually simpler. In all cases, the diagrammatic estimates for $\pi^{(N)}$ are ultimately very similar, and these are briefly discussed in Section 13.5. 


\subsection{Inclusion-Exclusion}

In this section, we derive the expansion (13.1) using the method of Sakai [179].

We write $\{(x, n) \Rightarrow(y, m)\}$ to denote the event that there are at least two bond-disjoint connections from $(x, n)$ to $(y, m)$, or that $(x, n)=(y, m)$. As in Definition $9.13(\mathrm{c})$, given a bond $b$ we define $\tilde{C}^{b}(x, n)$ to be the random set of vertices to which $(x, n)$ remains connected after the bond $b$ is made vacant.

As in (10.8), the first step of the expansion is to partition the event $\{(0,0) \rightarrow(x, n)\}$ according to whether or not there is a double connection. This gives

$$
\tau_{n}(x)=\mathbb{P}((0,0) \Rightarrow(x, n))+\mathbb{P}((0,0) \rightarrow(x, n),(0,0) \not(x, n)) .
$$

In the last term, there must be an occupied pivotal bond for the connection, and hence a first such bond. Given a bond $b=\{(u, n),(v, n+1)\}$, let $\bar{b}=$ $(v, n+1)$ be the "top" of $b$, and $\underline{b}=(u, n)$ be the "bottom" of $b$. Partitioning according to the first pivotal bond gives

$$
\begin{aligned}
& \mathbb{P}((0,0) \rightarrow(x, n),(0,0) \nRightarrow(x, n)) \\
& =\sum_{b_{1}} \mathbb{P}\left((0,0) \Rightarrow \underline{b}_{1} \rightarrow \bar{b}_{1} \rightarrow(x, n),(x, n) \notin \tilde{C}^{b_{1}}(0,0)\right),
\end{aligned}
$$

where the condition $(x, n) \notin \tilde{C}^{b_{1}}(0,0)$ ensures that $b_{1}$ is pivotal. We use inclusion-exclusion for this condition, and then the Markov property, to obtain

$$
\begin{aligned}
& \mathbb{P}\left((0,0) \Rightarrow \underline{b}_{1} \rightarrow \bar{b}_{1} \rightarrow(x, n),(x, n) \notin \tilde{C}^{b_{1}}(0,0)\right) \\
&= \mathbb{P}\left((0,0) \Rightarrow \underline{b}_{1} \rightarrow \bar{b}_{1} \rightarrow(x, n)\right) \\
&-\mathbb{P}\left((0,0) \Rightarrow \underline{b}_{1} \rightarrow \bar{b}_{1} \rightarrow(x, n),(x, n) \in \tilde{C}^{b_{1}}(0,0)\right) \\
&= \mathbb{P}\left((0,0) \Rightarrow \underline{b}_{1}\right) p \mathbb{P}\left(\bar{b}_{1} \rightarrow(x, n)\right) \\
&-\mathbb{P}\left((0,0) \Rightarrow \underline{b}_{1} \rightarrow \bar{b}_{1} \rightarrow(x, n),(x, n) \in \tilde{C}^{b_{1}}(0,0)\right) .
\end{aligned}
$$

To rewrite (13.4), we define

$$
\pi_{m}^{(0)}(x)=\mathbb{P}_{p}((0,0) \Rightarrow(x, m))-\delta_{0, x} \delta_{0, m},
$$

and note that $\pi_{0}^{(0)}(x)=\pi_{1}^{(0)}(x)=0$ by definition. For $n \geq 1$, the combination of (13.2)-(13.4) can be written as

$$
\begin{aligned}
\tau_{n}(x)= & \pi_{n}^{(0)}(x)+\left(p|\Omega| D * \tau_{n-1}\right)(x)+\sum_{m=2}^{n-1}\left(\pi_{m}^{(0)} * p|\Omega| D * \tau_{n-1-m}\right)(x) \\
& -\sum_{b_{1}} \mathbb{P}\left((0,0) \Rightarrow \underline{b}_{1} \rightarrow \bar{b}_{1} \rightarrow(x, n),(x, n) \in \tilde{C}^{b_{1}}(0,0)\right) .
\end{aligned}
$$


Note that if the last line of (13.6) is ignored, this is (13.1) with $\pi$ replaced by $\pi^{(0)}$.

The expansion continues with the last term of (13.6). To understand this term, the concept of backbone is useful. Given a configuration in which $\bar{b}_{1}$ is connected to $(x, n)$, the backbone is defined to consist of the vertices on occupied paths from $\bar{b}_{1}$ to $(x, n)$ (including $\bar{b}_{1}$ and $\left.(x, n)\right)$. The event $\{(x, n) \in$ $\left.\tilde{C}^{b_{1}}(0,0)\right\}$ is equivalent to the existence of a vertex $(y, m) \in \tilde{C}^{b_{1}}(0,0)$ in the backbone. An important event is that all such vertices occur after any pivotal bonds for $\bar{b}_{1} \rightarrow(x, n)$. This motivates the following definition.

Given a bond $b$, a vertex $(x, n)$, and a random or deterministic set $A$ of vertices, let $E(b,(x, n) ; A)$ denote the event that:

- $b$ is occupied, and

- there exists a vertex $(y, m) \in A$ such that $\bar{b} \rightarrow(y, m) \rightarrow(x, n)$, and

- there is no pivotal bond $b^{\prime}$ for the connection from $\bar{b}$ to $(x, n)$ such that the backbone joining $\bar{b}$ to $\underline{b}^{\prime}$ contains a vertex of $A$.

This event plays a key role in the development of the expansion. We write $\bar{b}<\bar{b}^{\prime}$ to mean that the temporal component of $\bar{b}$ is less than that of $\bar{b}^{\prime}$, and, similarly, we write $\bar{b}<n$ when the temporal component of $\bar{b}$ is less than $n$.

Exercise 13.1. Let $w \in \mathbb{Z}^{d}$, and suppose that $l<\bar{b} \leq n$. Show that the above event is related to the event of (10.11) by

$$
E\left(b,(x, n) ; \tilde{C}^{b}(w, l)\right)=\{b \text { is occupied }\} \cap E^{\prime}\left(\bar{b},(x, n) ; \tilde{C}^{b}(w, l)\right),
$$

where here the event $\tilde{C}^{b}(w, l)$ belongs to the same probability space as the events $E$ and $E^{\prime}$ (i.e., there is a single percolation model and not multiple models as in Section 10.1).

Lemma 13.2. Let $b$ be a bond, let $F$ be an event that depends only on bonds $(u, j)$ with $j \leq \underline{b}$, let $(v, k)$ be a vertex with $k \leq \underline{b}$, let $(x, n)$ be a vertex with $\bar{b} \leq n$, and, given any vertex $(y, m)$ with $\bar{b} \leq m$, let $F_{(y, m)}=F \cap$ $E\left(b,(y, m) ; \tilde{C}^{b}(v, k)\right)$. Then

$$
\begin{aligned}
\mathbb{P}(F & \left.\cap\{\underline{b} \rightarrow \bar{b} \rightarrow(x, n)\} \cap\left\{(x, n) \in \tilde{C}^{b}(v, k)\right\}\right) \\
= & \mathbb{P}\left(F_{(x, n)}\right)+\sum_{b^{\prime}} \mathbb{P}\left(F_{\underline{b}^{\prime}}\right) p \mathbb{P}\left(\bar{b}^{\prime} \rightarrow(x, n)\right) \\
& -\sum_{b^{\prime}} \mathbb{P}\left(F_{\underline{b}^{\prime}} \cap\left\{\underline{b}^{\prime} \rightarrow \bar{b}^{\prime} \rightarrow(x, n)\right\} \cap\left\{(x, n) \in \tilde{C}^{b^{\prime}}(\bar{b})\right\}\right) .
\end{aligned}
$$

Proof. We partition the event on the left hand side according to the first pivotal bond $b^{\prime}$ for $\bar{b} \rightarrow(x, n)$, if there is one, such that the event $E\left(\bar{b}, \underline{b}^{\prime} ; \tilde{C}^{b}(v, k)\right)$ occurs. If there is no such pivotal bond, then the event $E\left(\bar{b},(x, n) ; \tilde{C}^{b}(v, k)\right)$ occurs, producing the first term on the right hand side of (13.8). The remaining contribution is 


$$
\sum_{b^{\prime}} \mathbb{P}\left(F_{\underline{b}^{\prime}} \cap\left\{\underline{b}^{\prime} \rightarrow \bar{b}^{\prime} \rightarrow(x, n)\right\} \cap\left\{(x, n) \notin \tilde{C}^{b^{\prime}}(\bar{b})\right\}\right) .
$$

After using inclusion-exclusion and then the Markov property on the last event in (13.9), this gives (13.8).

Note that the summand in the last term on the right hand side of (13.8) is of the same form as the left hand side, so the identity can be iterated. The iteration begins with the last term on the right hand side of (13.4), whose summand is equal to the left hand side of (13.8) with $F=\left\{(0,0) \Rightarrow \underline{b}_{1}\right\}$. To record the result of the iteration, we make the following definitions. For $N \geq 1$, let

$$
B_{N}(n)=\left\{\vec{b}=\left(b_{1}, \ldots, b_{N}\right): 0<\bar{b}_{1}<\cdots<\bar{b}_{N} \leq n\right\}
$$

denote the ordered vectors of $N$ bonds, up to time $n$. Given $\vec{b} \in B_{N}(n)$, we define $\bar{b}_{0}=(0,0), \underline{b}_{N+1}=(x, n)$. For $N \geq 1$, we define

$$
\pi_{m}^{(N)}(x)=\sum_{\vec{b} \in B_{N}(m)} \mathbb{P}_{p}\left[\left\{(0,0) \Rightarrow \underline{b}_{1}\right\} \cap \bigcap_{i=1}^{N} E\left(\bar{b}_{i}, \underline{b}_{i+1} ; \tilde{C}^{b_{i}}\left(\bar{b}_{i-1}\right)\right)\right]
$$

Also, we define

$$
\pi_{m}(x)=\sum_{N=0}^{\infty}(-1)^{N} \pi_{m}^{(N)}(x),
$$

where the apparently infinite sum is actually a finite sum, since the sum in (13.11) is empty if $N>m$, in which case $\pi_{m}^{(N)}(x)=0$.

The last term of (13.6) is then computed iteratively using Lemma 13.2, to produce the expansion (13.1). The iteration eventually terminates because the remainder term in (13.8) will vanish after the number of iterations exceeds $n$.

\subsection{Laces and Resummation}

In this section, we derive the expansion of Nguyen and Yang [166]. This expansion has been applied also in $[167,121]$. The method is closely related to the lace expansion for lattice trees and lattice animals, and uses the notion of connected graph and lace from Section 8.1.1.

For $t=0,1,2, \ldots$, we define $W_{n, t}(x)$ to be the event that $(0,0) \rightarrow(x, n)$ with exactly $t$ occupied pivotal bonds for the connection, and let

$$
\tau_{n, t}(x)=\mathbb{P}_{p}\left(W_{n, t}(x)\right) .
$$

By definition, $\tau_{n}(x)=\sum_{t=0}^{\infty} \tau_{n, t}(x)$. We will rewrite $\tau_{n, t}(x)$ in terms of a repulsive interaction between the sausages in the string of sausages representing the connection $(0,0) \rightarrow(x, n)$ (see Fig. 10.1). When $W_{n, t}(x)$ occurs, there are exactly $t+1$ sausages. 
Recalling the definition of $B_{t}(n)$ in (13.10), for $t \geq 1$ and $\vec{b} \in B_{t}(n)$ we define

$$
T(\vec{b},(x, n))=\bigcap_{i=1}^{t}\left\{b_{i} \text { occupied }\right\} \bigcap_{i=0}^{t}\left\{\bar{b}_{i} \Rightarrow \underline{b}_{i+1}\right\} .
$$

Note that if $T(\vec{b},(x, n))$ occurs, then the only possible candidates for occupied pivotal bonds for the event $(0,0) \rightarrow(x, n)$ are the elements of $\vec{b}$ (but these candidates need not be pivotal). We define the random variables

$$
K[i, j]=\prod_{i \leq s<t \leq j}\left(1+U_{s t}\right) \quad \text { with } \quad U_{i j}=-I\left[\bar{b}_{i} \Rightarrow \underline{b}_{j+1}\right] .
$$

The product in (13.15) is 0 or 1 . The event that both $T(\vec{b},(x, n))$ occurs and $K[0, t]=1$ is the event that the occupied pivotal bonds for $(0,0) \rightarrow(x, n)$ are precisely the elements of $\vec{b}$. Therefore, for $t \geq 1$,

$$
\tau_{n, t}(x)=\sum_{\vec{b} \in B_{t}(n)} \mathbb{E}_{p}[I[T(\vec{b},(x, n))] K[0, t]] .
$$

Using the terminology of graphs and connected graphs defined in Section 8.1.1, we have

$$
K[a, b]=\sum_{\Gamma \in \mathcal{B}[a, b]} \prod_{i j \in \Gamma} U_{i j}
$$

with $K[a, a]=1$. Let

$$
J[a, b]=\sum_{\Gamma \in \mathcal{G}[a, b]} \prod_{i j \in \Gamma} U_{i j} .
$$

These are related by (8.6), which states that for $t \geq 1$,

$$
K[0, t]=K[1, t]+\sum_{s=1}^{t-1} J[0, s] K[s+1, t]+J[0, t],
$$

where the empty sum is zero if $t=1$. Let

$$
\begin{aligned}
& \pi_{m, 0}(x)=\mathbb{P}_{p}((0,0) \Rightarrow(x, m))-\delta_{0, x} \delta_{0, m}, \\
& \pi_{m, s}(x)=\sum_{\vec{b} \in B_{s}(m)} \mathbb{E}_{p}[I[T(\vec{b},(x, m))] J[0, s]] \quad(s \geq 1) .
\end{aligned}
$$

It can be seen from the above definitions that $\pi_{m, 0}(x)=\tau_{m, 0}(x)$ if $m>0$, and that $\pi_{m, s}(x)=0$ whenever $m=0,1$ or $s>m$. Let

$$
\pi_{m}(x)=\sum_{s=0}^{\infty} \pi_{m, s}(x)=\sum_{s=0}^{m} \pi_{m, s}(x) .
$$

Substitution of (13.19) into (13.16), followed by application of the Markov property, then gives the recursion formula 


$$
\begin{aligned}
\tau_{n, t}(x)= & \left(p|\Omega| D * \tau_{n-1, t-1}\right)(x)+\sum_{m=2}^{n-1} \sum_{s=0}^{t-1}\left(\pi_{m, s} * p|\Omega| D * \tau_{n-1-m, t-1-s}\right)(x) \\
& +\pi_{n, t}(x),
\end{aligned}
$$

valid for all $n, t \geq 1$. Summation of (13.23) over $t \geq 1$, and using $\pi_{n, 0}(x)=$ $\tau_{n, 0}(x)$ for $n \geq 1$, then gives

$$
\tau_{n}(x)=\left(p|\Omega| D * \tau_{n-1}\right)(x)+\sum_{m=2}^{n-1}\left(\pi_{m} * p|\Omega| D * \tau_{n-1-m}\right)(x)+\pi_{n}(x)
$$

for $n \geq 1$. The identity (13.24) is identical to (13.1).

To obtain a useful representation for $\pi_{m}(x)$, we rewrite $\pi_{m, s}(x)$ in terms of laces. Insertion of (8.5) into (13.21) leads to

$$
\pi_{m, s}(x)=\sum_{N=0}^{\infty}(-1)^{N} \pi_{m, s}^{(N)}(x)
$$

with $\pi_{m, s}^{(0)}(x)=\pi_{m, 0}(x) \delta_{0, s}$, and, for $N \geq 1$,

$$
\begin{aligned}
\pi_{m, s}^{(N)}(x)=\sum_{\vec{b} \in B_{s}(m)} \mathbb{E}_{p}[I[T(\vec{b},(x, m))] & \\
& \left.\times \sum_{L \in \mathcal{L}^{(N)}[0, s]} \prod_{i j \in L}\left(-U_{i j}\right) \prod_{i^{\prime} j^{\prime} \in \mathcal{C}(L)}\left(1+U_{i^{\prime} j^{\prime}}\right)\right]
\end{aligned}
$$

(the right hand side is interpreted as 0 when $s=0$ ). The product $\prod_{i j \in L}\left(-U_{i j}\right)$ is either 0 or 1 , so $\pi_{m, s}^{(N)}(x) \geq 0$. The above gives the decomposition

$$
\pi_{m}(x)=\sum_{N=0}^{\infty}(-1)^{N} \pi_{m}^{(N)}(x) \quad \text { with } \quad \pi_{m}^{(N)}(x)=\sum_{s=0}^{\infty} \pi_{m, s}^{(N)}(x)
$$

With some effort, it can be seen that $\pi_{m}^{(N)}$ of $(13.26)-(13.27)$ is the same as $\pi_{m}^{(N)}$ of (13.11).

\subsection{Application of the Percolation Expansion}

Recall that the expansion

$\tau(0, y)=\delta_{0, y}+(p|\Omega| D * \tau)(0, y)+\left(\Pi_{M} * p|\Omega| D * \tau\right)(0, y)+\Pi_{M}(0, y)+R_{M}(0, y)$,

of (13.28), with $\Pi_{M}$ and $R_{M}$ given by (10.28)-(10.29), applies to an arbitrary regular graph. In the derivation of (13.28), bonds were not directed. However, the modifications due to orientation are simply notational, as we now explain. 
For oriented percolation on $\mathbb{Z}^{d} \times \mathbb{Z}_{+}$, the variables 0 and $y$ in (13.28) now correspond to space-time vertices $(0,0)$ and $(x, n)$ (say). The convolution in (13.28) is a convolution on $\mathbb{Z}^{d} \times \mathbb{Z}_{+}$, and thus involves both spatial and temporal convolution.

The left hand side of (13.28) is simply the two-point function $\tau_{n}(x)$. We assume that $n \geq 1$, so the Kronecker delta on the right hand side of (13.28) is zero. In an abuse of notation, the function $D$ in (13.28), which is a function on pairs $((u, j),(v, j+1))$, will be also written as $D(v-u)$. Thus, noting that the factor $D$ consumes exactly one unit of time, the first convolution term in (13.28) can be written as the spatial convolution

$$
p|\Omega|\left(D * \tau_{n-1}\right)(x) .
$$

All connections in oriented percolation between distinct vertices involve a positive time difference between those vertices. In the definition of the remainder term $R_{M}((0,0),(x, n))$ in (10.29), there is a sum over oriented bonds $\left(\left(u_{i}, m\right),\left(v_{i}, m+1\right)\right)$, and the time associated with $v_{i}$ cannot be more than the time associated with $u_{i+1}$, since events in (10.29) require connections from the former to the latter. The overall summation in (10.29) is therefore empty when $M>n$. The same is true for $\Pi^{(N)}$ for $N>m$, and we may simply take $M=\infty$ in (13.28), and drop the remainder term. Writing $\pi=\Pi_{M=\infty}$, we then have

$$
\left(\Pi_{M} * p|\Omega| D * \tau\right)(0, y)+\Pi_{M}(0, y)=\sum_{m=2}^{\infty}\left(\pi_{m} * p|\Omega| D * \tau_{n-m-1}\right)(x)+\pi_{n}(x)
$$

(convolutions on the left are space-time and on the right space only). Thus (13.28) becomes (13.1).

The representations for $\Pi^{(N)}$ and $\pi^{(N)}$ are quite different and there is no apparent reason to expect them to be equal, even though their alternating sums must agree.

\subsection{The Upper Critical Dimension}

It is interesting to reinterpret for oriented percolation the explanation of the upper critical dimension given at the end of Section 10.1 for ordinary percolation. For oriented percolation, the approximation in which $\tau^{\tilde{C}^{\{u, v\}}(0)}(v, x)$ is replaced by by $\tau(v, x)$ is a good approximation when the backbone joining $v$ and $x$ typically does not have a space-time intersection with the cluster $\tilde{C}^{\{u, v\}}(0)$. Assuming Gaussian scaling limits, the backbone corresponds to a Brownian path, whereas the cluster $\tilde{C}^{\{u, v\}}(0)$ corresponds to a superBrownian cluster (see Section 17.3). The upper critical dimension can then be understood as the dimension above which the graphs of Brownian motion and super-Brownian motion do not intersect.

Intersection of the graphs implies a collision of the two processes at the same time. It is known that $d=4$ is critical for such a collision [19]. This can 
be understood heuristically by the following very rough argument. We first assume that since both processes are moving randomly it is reasonable to treat one of them as being stationary (this is a leap of faith). Regarding the superBrownian motion as stationary, its support at fixed time is 2-dimensional. The Brownian path, which is two-dimensional, will generically not hit this support in dimensions greater than $4=2+2$. Alternately, if we regard the Brownian motion as being fixed, then its support is a point, hence 0-dimensional. The 4-dimensional range of super-Brownian motion will generically not hit this point in dimensions above $4=4+0$. Either way, this points to 4 as the upper critical dimension.

\subsection{Diagrams for Oriented Percolation}

If the representation for $\pi_{m}$ of Section 13.3 is used, then $\pi_{m}$ is bounded by diagrams precisely as in Section 10.2, with the understanding that now the two-point function is that of oriented percolation rather than ordinary percolation. These oriented percolation diagrams can then be estimated using the method of Section 10.3, for example, taking into account the orientation. There are different possibilities for how to proceed with this, and detailed estimates can be found in $[119,121,166,179]$.

It is worth noting that the representations for $\pi_{m}$ of Section 13.1 and 13.2 can be estimated by diagrams that, although similar, are simpler than the ordinary percolation diagrams of Section 10.2. For example, consider $\pi_{m}^{(1)}(x)$ of (13.11). It can be concluded from the definition of the event $E\left(\left(\bar{b}_{1},(x, m) ; \tilde{C}^{b_{1}}(0)\right)\right.$ that the event

$$
\left\{(0,0) \Rightarrow \underline{b}_{1}\right\} \cap E\left(\left(\bar{b}_{1},(x, m) ; \tilde{C}^{b_{1}}(0)\right)\right.
$$

is a subset of the event that there exists a vertex $(z, l)$ and such that the following connections occur disjointly: $(0,0) \rightarrow(z, l),(z, l) \rightarrow \underline{b}_{1},(0,0) \rightarrow \underline{b}_{1}$, $\underline{b}_{1} \rightarrow(x, n)$, and $(z, l) \rightarrow(x, n)$. Therefore $\pi_{m}^{(1)}(x)$ is bounded above by the left diagram in Fig. 13.1. It can similarly be concluded that $\pi_{m}^{(2)}(x)$ is bounded by the diagrams shown in Fig. 13.1, and this can be extended in a straightforward manner to obtain diagrams bounding $\pi_{m}^{(N)}(x)$ for larger values of $N$.

\section{The Contact Process}

The contact process is a much-studied model of the spread of infection, first introduced in [107]. For an introduction, see the books [153, 154]. In this

section, we survey results that have been obtained for the critical behaviour of the contact process above its upper critical dimension $d=4$. 


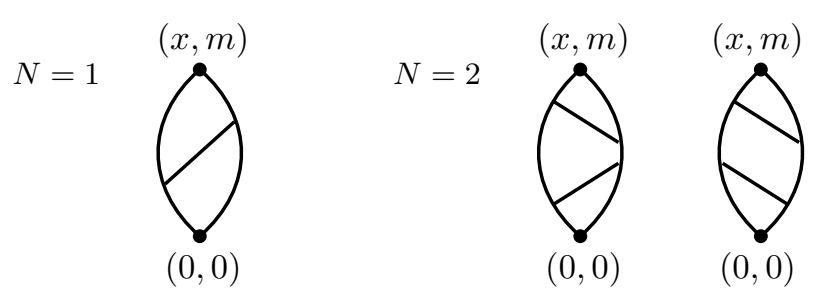

Fig. 13.1. Diagrams bounding $\pi_{m}^{(1)}(x)$ and $\pi_{m}^{(2)}(x)$.

\subsection{The Phase Transition}

The contact process is a continuous-time Markov process with state space $\{0,1\}^{\mathbb{Z}^{d}}$, with $d \geq 1$. In particular, the state of the contact process is determined by a variable $\xi_{x} \in\{0,1\}$, for each vertex $x \in \mathbb{Z}^{d}$. When $\xi_{x}=0$, then $x$ is considered "healthy," and when $\xi_{x}=1$, then $x$ is considered "infected." Infected particles spontaneously become healthy at rate 1 , and healthy particles become infected at a rate proportional to their number of infected neighbours, i.e., at a rate $\lambda \sum_{y \in \Omega} \xi_{x+y}$, where we will assume that $\Omega$ is given either by (1.1) or (1.2).

The contact process has the convenient graphical representation depicted in Fig. 14.1. In the graphical representation, to each vertex there is associated a Poisson process of rate 1 called the recovery process, and to each directed bond $(x, y)$ (with $y-x \in \Omega$ ) there is associated a Poisson process of rate $\lambda$ called the infection process. All the Poisson processes are independent. Recovery marks are placed on the times lines of each vertex according to the recovery process at that vertex. Infection arrows are drawn from $x$ to $y$ at the times of the infection process associated to $(x, y)$. As time proceeds, an infected particle at $x$ becomes healthy at the recovery marks (nothing happens to a healthy particle at these times), and an infection arrow $(x, y)$ causes $y$ to become infected if $x$ is infected. Thus, starting with an infected particle, infection proceeds along directed paths from that particle which follow time lines in the direction of increasing time, which do not cross recovery marks, and which follow infection arrows in the direction of the arrow.

We are interested in the situation in which at time zero there is a single infected particle at the origin and all other particles are healthy. The main question asks whether there is a positive probability that the infection survives for all time, or whether instead all particles are eventually healthy with probability one. The basic fact is that there is a critical value $\lambda_{c} \in(0, \infty)$ such that the infection dies out with probability one if $\lambda<\lambda_{c}$ but has a positive probability of survival if $\lambda>\lambda_{c}$. A theorem of Bezuidenhout and Grimmett [22] (see also [86] for the spread-out model) states that the infection dies out with probability one at the critical value $\lambda=\lambda_{c}$. 


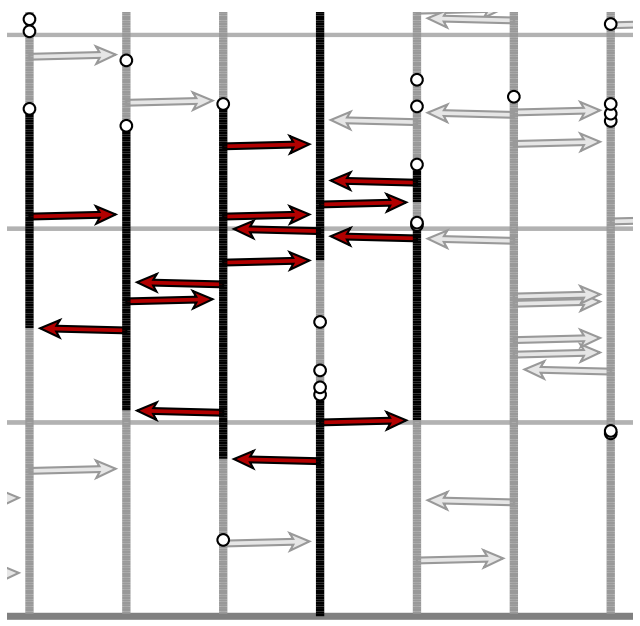

Fig. 14.1. The graphical representation of the contact process on $\mathbb{Z}^{1}$, with a single infected particle at time 0 . Time increases in the upward direction.

The phase transition can be studied in terms of several functions familiar from percolation. Let $C_{t}$ denote the set of infected particles at time $t$. The two-point function is defined by

$$
\tau_{\lambda, t}(x)=\mathbb{P}_{\lambda}\left(x \in C_{t}\right),
$$

for $x \in \mathbb{Z}^{d}$ and $t \geq 0$. The susceptibility is defined by

$$
\chi(\lambda)=\int_{0}^{\infty} \mathrm{d} t \hat{\tau}_{\lambda, t}(0)=\mathbb{E}_{\lambda} \int_{0}^{\infty} \mathrm{d} t\left|C_{t}\right|=\mathbb{E}_{\lambda}\|C\|,
$$

where $\|C\|=\int_{0}^{\infty} \mathrm{d} t\left|C_{t}\right|$ is the total Lebesgue measure of all infection intervals on all time lines. The spread probability is defined by

$$
\theta(\lambda)=\mathbb{P}_{\lambda}\left(C_{t} \neq \varnothing, \forall t \geq 0\right)=\mathbb{P}_{\lambda}(\|C\|=\infty) .
$$

It is known [23] that the critical point has the dual characterization

$$
\lambda_{c}=\sup \{\lambda: \chi(\lambda)<\infty\}=\inf \{\lambda: \theta(\lambda)>0\} .
$$

The magnetization is defined by

$$
M_{\lambda}(h)=1-\mathbb{E}_{\lambda}\left(\mathrm{e}^{-h\|C\|}\right) .
$$

Critical exponents are defined, as usual, by

$$
\begin{aligned}
\chi(\lambda) & \sim c\left(\lambda_{c}-\lambda\right)^{-\gamma} \quad \text { as } \lambda \rightarrow \lambda_{c}^{-}, \\
M_{\lambda_{c}}(h) & \sim c h^{1 / \delta} \quad \text { as } h \rightarrow 0, \\
\theta(\lambda) & \sim c\left(\lambda-\lambda_{c}\right)^{\beta} \quad \text { as } \lambda \rightarrow \lambda_{c}^{+} .
\end{aligned}
$$


The critical exponents are predicted to be universal, and it is an important problem to prove that they exist and to calculate their values. The mean-field values, corresponding to the contact process on a regular tree or to branching random walk, are $\gamma=\beta=1$ and $\delta=2$. The mean-field bounds

$$
\begin{aligned}
\chi(\lambda) & \geq c\left(\lambda_{c}-\lambda\right)^{-1}, \\
M_{\lambda_{c}}(h) & \geq c h^{1 / 2}, \\
\theta(\lambda) & \geq c\left(\lambda-\lambda_{c}\right)
\end{aligned}
$$

are proved in [23] (for $\beta$ and $\delta$ ) and in [21] (for $\gamma$ ), for all $d \geq 1$. The hyperscaling inequalities proved in [180] imply that the upper critical dimension for the contact process is at least 4 , in the sense that not all critical exponents can assume their mean-field values in dimensions less than 4 (assuming the exponents exist).

\subsection{Approximation by Oriented Percolation}

The contact process can be approximated by an oriented percolation model. This observation has been an important ingredient in the work of many authors, including $[21,23,117,119,179]$.

To discretize time, we replace the time interval $[0, \infty)$ by $\epsilon \mathbb{Z}_{+}$, and define an oriented percolation model on $\mathbb{Z}^{d} \times \in \mathbb{Z}_{+}$, as follows. Bonds have the form $((x, t),(y, t+\epsilon))$ with $t \in \epsilon Z_{+}$and $y-x \in\{0\} \cup \Omega$. A bond is occupied with probability $1-\epsilon$ if $x=y$, and with probability $\epsilon \lambda$ if $y-x \in \Omega$. Bonds with $x=y$ are called temporal and bonds with $x \neq y$ are called spatial. See Fig. 14.2.

Let $\mathbb{P}_{\lambda}^{\epsilon}$ denote the probability measure for the oriented percolation model on $\mathbb{Z}^{d} \times \epsilon \mathbb{Z}_{+}$. Then $\mathbb{P}_{\lambda}^{\epsilon}$ converges ${ }^{14}$ weakly as $\epsilon \rightarrow 0^{+}$to the contact process measure $\mathbb{P}_{\lambda}$ (when formulated appropriately), and the critical value $\lambda_{c}^{\epsilon}$ of the discretized model converges to the critical value $\lambda_{c}$ of the contact process (see $[23,179])$.

This provides an avenue for analyzing the contact process by first analyzing an oriented percolation model on $\mathbb{Z}^{d} \times \epsilon \mathbb{Z}_{+}$and then taking the limit $\epsilon \rightarrow 0^{+}$. In particular, the expansions of Section 13 for oriented percolation can be applied to the discretized model.

\subsection{The Infrared Bound and the Triangle Condition}

Let

$$
\hat{\tau}_{\lambda}(k, \theta)=\sum_{x \in \mathbb{Z}^{d}} \int_{0}^{\infty} \mathrm{d} t \tau_{\lambda, t}(x) \mathrm{e}^{\mathrm{i} k \cdot x} \mathrm{e}^{\mathrm{i} \theta t},
$$

for $k \in[-\pi, \pi]^{d}$ and $\theta \in[-\pi, \pi]$. This is analogous to (12.15) with $z=\mathrm{e}^{\mathrm{i} \theta}$. The following infrared bound was proved in [179]. The proof uses discretization by

\footnotetext{
$\overline{{ }^{14} \text { Fig. } 14.1}$ is in fact a simulation of $\mathbb{P}_{\lambda}^{\epsilon}$ with small $\epsilon$.
} 


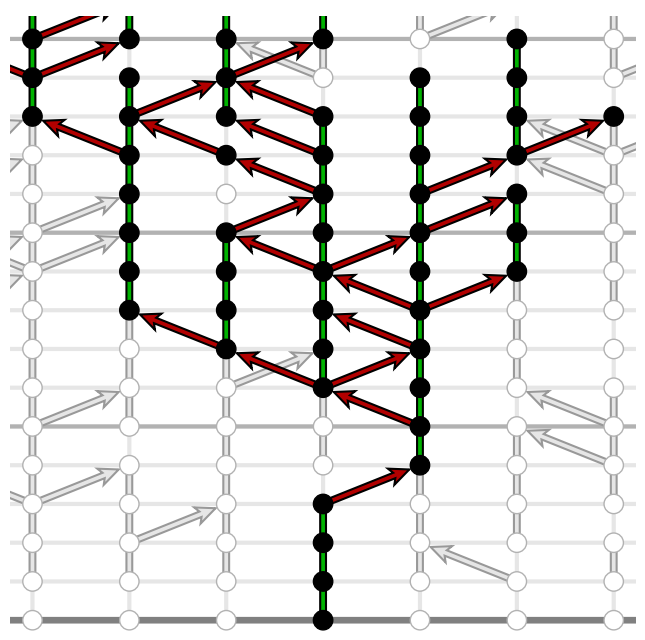

Fig. 14.2. Approximation of the contact process by oriented percolation on $\mathbb{Z}^{1} \times \mathbb{Z}_{+}$.

oriented percolation, the oriented percolation expansion of Section 13.1, and a convergence proof based on the bootstrap Lemma 5.9.

Theorem 14.1. There exist $d_{0} \gg 4$ and $L_{0}(d) \gg 1$ such that for the nearestneighbour contact process in dimensions $d \geq d_{0}$ or for the spread-out contact process in dimensions $d>4$ with $L \geq L_{0}(d)$, there is a constant $C$ (depending on $d$ and $L$ ) such that the infrared bound

$$
\left|\hat{\tau}_{\lambda}(k, \theta)\right| \leq \frac{C}{|k|^{2}+|\theta|}
$$

holds uniformly in $\lambda<\lambda_{c}, k \in[-\pi, \pi]^{d}$ and $\theta \in[-\pi, \pi]$.

The infrared bound implies the triangle condition for the contact process. To state the triangle condition, we define

$$
\nabla_{\lambda}(R)=\sup _{x:|x| \geq R, t \geq 0} \sum_{y, z} \int_{0}^{\infty} \mathrm{d} s_{1} \int_{0}^{\infty} \mathrm{d} s_{2} \tau_{\lambda, s_{1}}(y) \tau_{\lambda, s_{2}-s_{1}}(z-y) \tau_{\lambda, s_{2}-t}(z-x)
$$

The triangle condition is the statement that

$$
\lim _{R \rightarrow \infty} \nabla_{\lambda_{c}}(R)=0
$$

and it is proved in [21] that if the triangle condition holds then the exponents $\gamma, \beta, \delta$ exist and take their mean-field values, in the sense that there are upper bounds (with different constants) complementary to the lower bounds (14.9)-(14.11). As in Exercise 12.2, if $d>4$ then the infrared bound (14.13) 
implies the triangle condition. Therefore, under the high- $d$ hypotheses of Theorem 14.1, the critical exponents $\gamma, \beta, \delta$ exist (in the sense of $\simeq$ ) and take the mean-field values $\gamma=\beta=1$ and $\delta=2$.

For the spread-out model, this was extended in [119] to prove the following theorem. The proof makes use of the induction method of [120], adapted to incorporate uniformity in the discretization parameter $\epsilon$.

Theorem 14.2. Consider the spread-out model of the contact process. Let $d>4, \lambda=\lambda_{c}$ and $\delta \in\left(0,1 \wedge \frac{d-4}{2}\right)$. There is an $L_{0}=L_{0}(d)$ such that for $L \geq L_{0}$ there exist finite positive constants $v$ and $A$ (depending on $d$ and $L$ ) and $C_{1}, C_{2}$ (depending only on $d$ ) such that the following statements hold as $t \rightarrow \infty$ :

(a)

$$
\hat{\tau}_{\lambda_{c}, t}(k / \sqrt{v t})=A \mathrm{e}^{-|k|^{2} / 2 d}\left[1+O\left(|k|^{2} t^{-\delta}\right)+O\left(t^{-(d-4) / 2}\right)\right],
$$

$$
\frac{1}{\hat{\tau}_{\lambda_{c}, t}(0)} \sum_{x \in \mathbb{Z}^{d}}|x|^{2} \tau_{\lambda_{c}, t}(x)=v t\left[1+O\left(t^{-\delta}\right)\right]
$$

$$
C_{1} L^{-d} t^{-d / 2} \leq \sup _{x \in \mathbb{Z}^{d}} \tau_{\lambda_{c}, t}(x) \leq \mathrm{e}^{-t}+C_{2} L^{-d} t^{-d / 2},
$$

with the error estimate in (a) uniform in $k \in \mathbb{R}^{d}$ with $|k|^{2} / \log t$ sufficiently small.

An extension of Theorem 14.2 to $r$-point functions for all $r \geq 2$, due to [117], is discussed in Section 17.4.

As is typical when the induction method of [120] is applied, the results of [119] also include the statements that $A=1+O\left(L^{-d}\right)$, that $v=\sigma^{2}\left[1+O\left(L^{-d}\right)\right]$ where $\sigma^{2}=\sum_{x}|x|^{2} D(x)$ is the variance of $D$, and that the critical value obeys $\lambda_{c}=|\Omega|^{-1}\left[1+O\left(L^{-d}\right)\right]$. The latter is improved in $[118]^{15}$ to

$$
\lambda_{c}=\frac{1}{|\Omega|}\left[1+\frac{1}{L^{d}} \sum_{n=2}^{\infty} U^{* n}(0)+O\left(L^{-(d+1)}\right)\right],
$$

where $U$ is the probability density function of a uniform random variable on $[-1,1]^{d}$ and $U^{* n}$ denotes its $n$-fold convolution. This improves a result of [68], where (14.19) was obtained with error term $o\left(|\Omega|^{-1}\right)=o\left(L^{-d}\right)$ by completely different methods. On the other hand, the results of [68] gives estimates for $\lambda_{c}$ also in dimensions $2 \leq d \leq 4$.

A related model is studied in $[68,119]$. In this model, the infection range $L$ is assumed to scale as

$$
L=L_{T}=L_{1} T^{b},
$$

$\overline{15}$ Note that $p_{c}$ in [118] is equal to our $\lambda_{c}|\Omega|$. 
where $b$ is a fixed parameter. This model allows for an analysis in dimensions $1 \leq d \leq 4$, provided $b$ is large enough. The result is consistent with the general philosophy that the upper critical dimension is lowered when the range of interaction is suitably increased. The following theorem is proved in [119].

Theorem 14.3. Let $1 \leq d \leq 4, b>\frac{4-d}{2 d}$, and fix $\delta \in\left(0,1 \wedge b d+\frac{d-4}{2}\right)$. Then there exist $\alpha>0, L_{0} \gg 1$, and a critical value $\lambda_{T}$, such that for $\lambda=\lambda_{T}$ and $L_{1} \geq L_{0}$ there exist $C_{1}, C_{2}$ (depending only on $d$ ) such that for all $0<t \leq$ $\log T$, as $T \rightarrow \infty$,

(a)

$$
\hat{\tau}_{\lambda_{T}, T t}\left(k / \sqrt{v_{T} T t}\right)=\mathrm{e}^{-|k|^{2} / 2 d}\left[1+O\left(|k|^{2}(1+T t)^{-\delta}\right)+O\left(T^{-\alpha}\right)\right],
$$

$$
\frac{1}{\hat{\tau}_{\lambda_{T}, T t}(0)} \sum_{x}|x|^{2} \tau_{\lambda_{T}, T t}(x)=v_{T} T t\left[1+O\left((1+T t)^{-\delta}\right)+O\left(T^{-\alpha}\right)\right],
$$

(c)

$$
C_{1} L_{T}^{-d}(1+T t)^{-d / 2} \leq \sup _{x \in \mathbb{Z}^{d}} \tau_{\lambda_{T}, T t}(x) \leq \mathrm{e}^{-T t}+C_{2} L_{T}^{-d}(1+T t)^{-d / 2},
$$

where $v_{T}$ is the variance of the range- $L_{T} D$. The error estimate in (14.16) is uniform in $k \in \mathbb{R}^{d}$ with $|k|^{2}(\log (2+T t))^{-1}$ sufficiently small.

The assumption on $b$ in Theorem 14.3 is

$$
b>\frac{4-d}{2 d}=\left\{\begin{array}{l}
3 / 2 \text { if } d=1 \\
1 / 2 \text { if } d=2 \\
1 / 6 \text { if } d=3 \\
0 \quad \text { if } d=4
\end{array}\right.
$$

Related results were obtained in [68] assuming $b=1$ in dimensions $d \geq 3$. On the other hand, in [68], for $d=2$ the weaker assumption $L_{T}=(T \log T)^{1 / 2}$ is required, whereas Theorem 14.3 requires $b>\frac{1}{2}$ when $d=2$.

To get some idea how the condition $b>\frac{4-d}{2 d}$ arises, consider the following. A quantity related to the critical triangle diagram (14.14) is

$$
\sum_{y, z} \int_{0}^{T \log T} \mathrm{~d} s_{2} \int_{0}^{s_{2}} \mathrm{~d} s_{1} \tau_{s_{1}}(y) \tau_{s_{2}-s_{1}}(z-y) \tau_{s_{2}}(z),
$$

where we consider $\lambda=\lambda_{T}$, and where we have taken $x=0$ and cut off the integral at the maximum value $T \log T$ allowed for $T t$ in Theorem 14.3. The estimates of Theorem 14.3 give good bounds on this quantity when $b>\frac{4-d}{2 d}$. In fact, using these estimates (with $k=0$ in (14.21)), (14.25) is at most a multiple of 


$$
\int_{0}^{T \log T} \mathrm{~d} s_{2} \int_{0}^{s_{2}} \mathrm{~d} s_{1}\left(e^{-s_{2}}+\frac{1}{L_{T}^{d}\left(1+s_{2}\right)^{d / 2}}\right) \leq O\left(1+T^{-b d}(T \log T)^{2-d / 2}\right),
$$

and the condition $b>\frac{4-d}{2 d}$ ensures that the right hand side is finite.

Remark 14.4. Theorems 14.2 and 14.3 are proved by obtaining versions of the theorems for the discretized model, with the estimates in the theorems uniform in $\epsilon \in(0,1]$. In particular, the results hold when $\epsilon=1$, which proves versions of the theorems, including Theorem 14.3, for the spread-out oriented percolation model with the usual unit time discretization.

\subsection{Expansion for the Contact Process}

In Section 13, we focussed attention on the nearest-neighbour and spreadout oriented percolation models, but the analysis holds more generally. In particular, the expansion (13.1) also applies to the oriented percolation model obtained by discretizing the contact process, when suitably interpreted. Two modifications are required to make (13.1) apply to the discretized contact process. The first is replacement of $p|\Omega| D(x)$ in the first two terms of the right hand side by

$$
p_{\lambda}^{\epsilon}(x)=\lambda \epsilon|\Omega| D(x)+(1-\epsilon) \delta_{x, 0},
$$

where the first and second terms on the right hand side correspond to the spatial and temporal bonds, respectively. The second modification is to interpret the definition of $\pi_{m}$ in terms of the oriented percolation model with both spatial and temporal bonds.

The expansion for the discretized contact process can then be written down immediately from (13.1), as

$$
\tau_{n \epsilon}^{\epsilon}(x)=\left(p^{\epsilon} * \tau_{(n-1) \epsilon}^{\epsilon}\right)(x)+\sum_{m=2}^{n-1}\left(\pi_{m \epsilon}^{\epsilon} * p^{\epsilon} * \tau_{(n-1-m) \epsilon}^{\epsilon}\right)(x)+\pi_{n \epsilon}^{\epsilon}(x) .
$$

Here, and in the following, we omit subscripts $\lambda$ from $\tau_{n \epsilon}^{\epsilon}(x), p^{\epsilon}(x)$ and $\pi_{m \epsilon}^{\epsilon}(x)$. If we consider momentarily the much simpler problem for which $\pi_{m \epsilon}^{\epsilon}$ is identically zero, then (14.28) becomes

$$
\tau_{n \epsilon}^{\epsilon}(x)=\lambda \epsilon|\Omega|\left(D * \tau_{(n-1) \epsilon}^{\epsilon}\right)(x)+(1-\epsilon) \tau_{(n-1) \epsilon}^{\epsilon}(x) .
$$

In terms of the Fourier transform, this can be rewritten as

$$
\frac{\hat{\tau}_{n \epsilon}^{\epsilon}(k)-\hat{\tau}_{(n-1) \epsilon}^{\epsilon}(k)}{\epsilon}=\lambda|\Omega| \hat{D}(k) \hat{\tau}_{(n-1) \epsilon}^{\epsilon}(k)-\hat{\tau}_{(n-1) \epsilon}^{\epsilon}(k) .
$$

In the limit $\epsilon \rightarrow 0^{+}$, where we also take $n \epsilon \rightarrow t$, this formally becomes the differential equation 


$$
\frac{\mathrm{d}}{\mathrm{d} t} \hat{\tau}_{t}(k)=-[1-\lambda|\Omega| \hat{D}(k)] \hat{\tau}_{t}(k) .
$$

With the initial condition $\hat{\tau}_{0}(k)=1,(14.31)$ has solution

$$
\hat{\tau}_{t}(k)=\mathrm{e}^{-[1-\lambda|\Omega| \hat{D}(k)] t} .
$$

For $\lambda=1 /|\Omega|$, this is the Fourier transform of the transition probability for continuous-time random walk which jumps at rate 1 .

If we now restore the omitted terms involving $\pi^{\epsilon}$ in (14.28), then the right hand side of (14.30) contains the additional terms

$$
\epsilon \sum_{m=2}^{n-1} \frac{1}{\epsilon^{2}} \hat{\pi}_{m \epsilon}^{\epsilon}(k) \hat{p}^{\epsilon}(k) \hat{\tau}_{(n-m-1) \epsilon}^{\epsilon}(k)+\frac{1}{\epsilon} \hat{\pi}_{n \epsilon}^{\epsilon}(k) .
$$

The factor $\epsilon$ has been placed in front of the summation to produce a Riemann sum. Since $\lim _{\epsilon \rightarrow 0} \hat{p}_{\lambda}^{\epsilon}(k)=1$, the limit of (14.33) apparently takes the form

$$
\int_{0}^{t} \hat{\pi}_{s}(k) \hat{\tau}_{t-s}(k) \mathrm{d} s
$$

with, for $m \epsilon \rightarrow s$ as $\epsilon \rightarrow 0$,

$$
\hat{\pi}_{s}(k)=\lim _{\epsilon \rightarrow 0} \frac{1}{\epsilon^{2}} \hat{\pi}_{m \epsilon}^{\epsilon}(k)
$$

(note that the last term of (14.33) vanishes in the limit if (14.35) holds.) Thus, provided it is possible to make sense of the limit (14.35), we expect that the contact process two-point function obeys the integro-differential equation

$$
\frac{\mathrm{d}}{\mathrm{d} t} \hat{\tau}_{t}(k)=-[1-\lambda|\Omega| \hat{D}(k)] \hat{\tau}_{t}(k)+\int_{0}^{t} \hat{\pi}_{s}(k) \hat{\tau}_{t-s}(k) \mathrm{d} s .
$$

A proof that this all works under the hypotheses of Theorem 14.2 or 14.3 is given in [119].

Equation (14.36) is a continuous-time version of the lace expansion, and it would be interesting to analyze it directly. However, such an analysis has not been carried out. Instead, in [119, 179], properties of the solution to (14.28) are analyzed directly, and estimates uniform in $\epsilon$ are obtained. The limit $\epsilon \rightarrow 0$ can then be taken to prove Theorems 14.1-14.3. A crucial step in the analysis is to prove that $\hat{\pi}_{m \epsilon}^{\epsilon}(k)$ is $O\left(\epsilon^{2}\right)$, consistent with (14.35). Next, we discuss the main idea that permits this.

\subsection{Diagrams for the Contact Process}

Now we describe the new observation needed to prove that $\hat{\pi}_{s}^{\epsilon}(k)$ is $O\left(\epsilon^{2}\right)$, consistent with (14.35), where we write $s=m \epsilon$. The diagrams for the discretized contact process are the same as the oriented percolation diagrams 
discussed in Section 13.5, with the understanding that temporal bonds now also occur.

The key observation already arises in the $N=0$ term, which is (cf. (13.5))

$$
\left(\hat{\pi}_{s}^{\epsilon}\right)^{(0)}(k)=\sum_{x \in \mathbb{Z}^{d}} \mathbb{P}_{\lambda}^{\epsilon}((0,0) \Rightarrow(x, s)) \mathrm{e}^{\mathrm{i} k \cdot x}
$$

for $s=m \epsilon>0$. Given that there are two disjoint connections from $(0,0)$ to $(x, s)$, and given that there is only one temporal bond leaving $(0,0)$ and one temporal bond entering $(x, s)$, it must be the case that one of the connections uses a spatial bond to leave $(0,0)$ and one must use a spatial bond to enter $(x, s)$. Thus there are two possibilities, depending on whether these two spatial bonds are part of the same connection or part of disjoint connections. This leads to the upper bound

$$
\begin{aligned}
& \lambda^{2}|\Omega|^{2} \epsilon^{2} \sum_{x \in \mathbb{Z}^{d}} \sum_{u \in \Omega} \sum_{x^{\prime} \in x-\Omega} D(u) D\left(x-x^{\prime}\right) \\
& \quad \times\left[\tau_{s-\epsilon}^{\epsilon}(x-u) \tau_{s-\epsilon}^{\epsilon}\left(x^{\prime}\right)+\tau_{s}^{\epsilon}(x) \tau_{s-2 \epsilon}^{\epsilon}\left(x^{\prime}-u\right)\right]
\end{aligned}
$$

for the absolute value of (14.37). If we now assume that the estimates (14.16) and (14.18) apply to the discretized model, and that $\lambda|\Omega|=O(1)$, then we can bound this above by $O\left(\epsilon^{2} L^{-d} s^{-d / 2}\right)$. In more detail, for the second term, we use (14.18) to bound $\tau_{s}^{\epsilon}(x)$ by $O\left(L^{-d} s^{-d / 2}\right)$, and then bound the sums using (14.16). Here we have used the theorem whose method of proof we are discussing, but this can be handed inductively.

For the higher-order diagrams that bound $\hat{\pi}_{s}^{\epsilon}(k)$, each diagram vertex contributes a factor $\epsilon$ due to the fact that there is only one temporal bond entering and leaving any given vertex. For all vertices other than the origin and the top vertex, this factor $\epsilon$ combines with the summation over the temporal coordinate of the vertex to produce an overall contribution $O(1)$. Thus, in the end, the upper bound involves $O\left(\epsilon^{2}\right)$. This procedure is carried out in detail in [119] and [179] for different forms of the diagrams (space-time diagrams and generating function diagrams, respectively).

\section{Branching Random Walk}

Branching random walk serves as the mean-field model for many interacting models involving branching, including lattice trees and percolation. In Section 15.1, we consider a natural mean-field model of lattice trees, which turns out to be intimately related to branching random walk with Poisson offspring distribution. In Section 15.2 we compute several generating functions important for a particular model of branching random walk. These will be used to derive the scaling limit of the branching random walk model in Section 16. In Section 15.3, we define a model of weakly self-avoiding lattice trees in terms of branching random walk. 


\subsection{A Mean-Field Model}

The mean-field model for the self-avoiding walk is simple random walk, which forgets about the self-avoidance interaction. It is natural to attempt to define a mean-field model of lattice trees by somehow forgetting about the mutual avoidance of the branches in a lattice tree. In this section, we define such a mean-field model, as in [61]. For simplicity, we consider only the nearestneighbour model.

It is convenient to switch to a site activity (or "fugacity"), rather than a bond activity. This means that we weight vertices rather than bonds by $z$, so that the one-point function of (7.20) becomes now $g(z)=G_{z}^{(1)}=$ $\sum_{T: T \ni 0} z^{|T|+1}$. If we remove the product containing the interaction in the two-point function (8.2), we obtain $\sum_{\omega \in \mathcal{W}(0, x)}(g(z))^{|\omega|+1}$. It is also useful to keep track of the length of the path $\omega$ by associating an activity $\zeta$ to each bond in this path. This prompts us to define the two-point function of the mean-field model by

$$
F_{z, \zeta}(x)=\sum_{\omega \in \mathcal{W}(0, x)}(f(z))^{|\omega|+1}\left(\frac{\zeta}{2 d}\right)^{|\omega|},
$$

where the function $f(z)$, to be specified below, is the one-point function for the mean-field model, and where the factor $\frac{1}{2 d}$ has been included as a convenient normalization. The sum in (15.1) is taken over simple random walks. Thus, $F_{z, \zeta}(x)$ can be written in terms of the simple random walk two-point function (1.17) as $F_{z, \zeta}(x)=f(z) C_{\zeta f(z) / 2 d}(0, x)$, and hence, by (1.18), its Fourier transform is

$$
\hat{F}_{z, \zeta}(k)=\frac{f(z)}{1-\zeta f(z) \hat{D}(k)},
$$

with $\hat{D}(k)$ given by (1.12). The random walk two-point function has critical value $\frac{1}{2 d}$, corresponding to $\zeta f(z)=1$. We will realize the latter with $\zeta=$ $f(z)=1$.

For the function $f(z)$, we require by analogy with (7.22) (taking into account the switch to site activity), that $f(z)$ satisfy the differential equation

$$
\hat{F}_{z, 1}(0)=z \frac{\mathrm{d} f(z)}{\mathrm{d} z} .
$$

Combining (15.3) and (15.2) gives

$$
\frac{f(z)}{1-f(z)}=z \frac{\mathrm{d} f(z)}{\mathrm{d} z} .
$$

Integrating the separable equation (15.4) over an interval $\left[z, z_{0}\right]$ gives

$$
\frac{f(z) \mathrm{e}^{-f(z)}}{f\left(z_{0}\right) \mathrm{e}^{-f\left(z_{0}\right)}}=\frac{z}{z_{0}} .
$$


The initial condition $z_{0}=1$ and $f\left(z_{0}\right)=1$ is a choice of normalization and gives

$$
f(z) \mathrm{e}^{-f(z)}=z \mathrm{e}^{-1} .
$$

By (15.6), $f$ can be written as $f(z)=-W\left(-z \mathrm{e}^{-1}\right)$, where $W$ is the principal branch of the Lambert $W$ function defined by $W(w) \mathrm{e}^{W(w)}=w[56]$. The latter is analytic on the $w$-plane with branch cut $\left(-\infty,-\mathrm{e}^{-1}\right.$, corresponding to a branch cut $[1, \infty)$ for $f(z)$ (and gives $f(0)=0$, as it should). This uniquely specifies $f(z)$, and hence $\hat{F}_{z, \zeta}(k)$. The same functions $f(z)$ and $\hat{F}_{z, \zeta}(k)$ will arise below in Theorem 15.2 for a model of branching random walk.

\subsection{Branching Random Walk}

In this section, we define a model of branching random walk in terms of embeddings of trees into $\mathbb{Z}^{d}$. The presentation is based on [30]. Some related ideas can be found in [36].

The trees are the family trees of the critical Galton-Watson branching process with Poisson offspring distribution. In more detail, we begin with a single individual having $\xi$ offspring, where $\xi$ is a Poisson random variable of mean 1, i.e., $\mathbb{P}(\xi=m)=(\mathrm{e} m !)^{-1}$. Each of the offspring then independently has offspring of its own, with the same critical Poisson distribution. To indicate when two trees are the same, we describe them in terms of words. The root is the word 0 . The children of the root are the words $01,02, \ldots 0 \xi_{0}$. The children of 01 are the words $011, \ldots, 01 \xi_{01}$, and so on. A tree is then uniquely represented by a set of words, and two trees are the same if and only if they are represented by the same set of words. A tree $T$ consisting of exactly $n$ individuals, with the $i^{\text {th }}$ individual having $\xi_{i}$ offspring, has probability

$$
\mathbb{P}(T)=\prod_{i \in T} \frac{1}{\mathrm{e} \xi_{i} !}=\mathrm{e}^{-n} \prod_{i \in T} \frac{1}{\xi_{i} !} .
$$

The product in (15.7) is over the vertices of $T$.

We define an embedding $\varphi$ of $T$ into $\mathbb{Z}^{d}$ to be a mapping from the vertices of $T$ into $\mathbb{Z}^{d}$, such that the root is mapped to the origin and adjacent vertices in the tree are mapped to nearest neighbours in $\mathbb{Z}^{d}$. There is no assumption that $\varphi$ is injective, and different vertices of $T$ can be mapped to the same vertex in $\mathbb{Z}^{d}$. Given a tree $T$ having $|T|$ vertices, there are $(2 d)^{|T|-1}$ possible embeddings $\varphi$ of $T$. A branching random walk configuration is then a pair $(T, \varphi)$, with associated probability

$$
\mathbb{P}(T, \varphi)=\frac{1}{(2 d)^{|T|-1}} \mathbb{P}(T) .
$$

Our aim in this section is to compute the $r$-point functions of this branching random walk model. These are generating functions for trees of fixed total number of vertices $n$, which visit a specified set of $r-1$ vertices in a specified manner (the $r^{\text {th }}$ point is the origin, where the embedding is rooted). 
We begin with the simplest case $r=1$. For $z \in \mathbb{C}$ with $|z| \leq 1$, the one-point function is defined by

$$
b_{z}^{(1)}=\sum_{(T, \varphi)} \mathbb{P}(T, \varphi) z^{|T|}=\sum_{T} \mathbb{P}(T) z^{|T|} .
$$

The series on the right hand side of (15.9) is the generating function for the probability mass function for the total size of a critical Poisson tree. It converges for $|z| \leq 1$, with $b_{1}^{(1)}=1$. For general $z, b_{z}^{(1)}$ is given in the following theorem.

We write $p_{m}=\mathbb{P}(\xi=m)=(\mathrm{e} m !)^{-1}$, and let

$$
P(w)=\sum_{m=0}^{\infty} p_{m} w^{m}=\mathrm{e}^{w-1}
$$

denote the generating function for the critical Poisson distribution.

Theorem 15.1. For $d \geq 1$, the one-point function is given by

$$
b_{z}^{(1)}=\sum_{n=1}^{\infty} \frac{n^{n-1}}{n !} \mathrm{e}^{-n} z^{n}
$$

and obeys

$$
b_{z}^{(1)} \mathrm{e}^{-b_{z}^{(1)}}=z \mathrm{e}^{-1} .
$$

Proof. Conditioning on the number of offspring of the root gives

$$
b_{z}^{(1)}=\sum_{m=0}^{\infty} p_{m} z\left(b_{z}^{(1)}\right)^{m}=z P\left(b_{z}^{(1)}\right)=z \mathrm{e}^{b_{z}^{(1)}-1},
$$

which implies (15.12). The Taylor expansion (15.11) then follows from Lagrange's inversion formula (see, e.g., [196, p.43]).

Since $b_{z}^{(1)}$ is real for real $z$ and $b_{0}^{(1)}=0$, it follows from (15.12) that $b_{z}^{(1)}$ is identical to the function $f(z)$ of the mean-field model of Section 15.1. Theorem 15.1 rederives the standard result that for the critical Poisson branching process,

$$
\mathbb{P}(|T|=n)=\frac{n^{n-1}}{n !} \mathrm{e}^{-n} .
$$

By Stirling's formula,

$$
\mathbb{P}(|T|=n) \sim \frac{1}{\sqrt{2 \pi}} \frac{1}{n^{3 / 2}} .
$$

Comparing with (7.2) and (7.3), this is a statement that the critical exponent $\theta$ takes the value $\theta=\frac{5}{2}$ for branching random walk.

The two-point function is a generating function for critical Poisson branching random walk which starts at the origin, which has a family tree whose 
total size is dual to an activity $z$, and which visits the vertex $x$ (possibly more than once) at a time dual to an activity $\zeta$. The two-point function is defined for $z, \zeta \in \mathbb{C}$ with $|z|<1,|\zeta| \leq 1$, and for $x \in \mathbb{Z}^{d}$, by

$$
b_{z, \zeta}^{(2)}(x)=\sum_{(T, \varphi)} \mathbb{P}(T, \varphi) z^{|T|} \sum_{i \in T} I[\varphi(i)=x] \zeta^{|i|},
$$

where $|i|$ denotes the graph distance from $i$ to the root of $T$. The series (15.16) clearly converges for $|z|<1,|\zeta| \leq 1$, as does its sum over $x \in \mathbb{Z}^{d}$. The following theorem gives the Fourier transform of the two-point function, and shows that it is identical to the mean-field two-point function of Section 15.1.

Theorem 15.2. For $d \geq 1, k \in[-\pi, \pi]^{d},|z|<1,|\zeta| \leq 1$,

$$
\hat{b}_{z, \zeta}^{(2)}(k)=\frac{b_{z}^{(1)}}{1-\zeta b_{z}^{(1)} \hat{D}(k)} .
$$

The denominator of the right hand side vanishes for $z=\zeta=1, k=0$, and in that case $\hat{b}_{1,1}^{(2)}(0)=\infty$.

Proof. The contribution to the right hand side of (15.16) arising when $i$ is the root is simply $b_{z}^{(1)} \delta_{0, x}$. When $i$ is not the root, we condition on the number of offspring of the root and on the location of the first step on the branch containing $i$, to obtain

$$
\begin{aligned}
b_{z, \zeta}^{(2)}(x) & =b_{z}^{(1)} \delta_{0, x}+\sum_{m=1}^{\infty} p_{m} m\left(b_{z}^{(1)}\right)^{m-1} z\left(\zeta D * b_{z, \zeta}^{(2)}\right)(x) \\
& =b_{z}^{(1)} \delta_{0, x}+z P^{\prime}\left(b_{z}^{(1)}\right)\left(\zeta D * b_{z, \zeta}^{(2)}\right)(x) .
\end{aligned}
$$

In the second term of the middle member of (15.18), the factor $z$ is associated with the root, and the factor $m$ corresponds to choosing which of the root's offspring is an ancestor of the vertex $j$. The Poisson generating function obeys $z P^{\prime}\left(b_{z}^{(1)}\right)=z P\left(b_{z}^{(1)}\right)$, and by (15.13), this equals $b_{z}^{(1)}$. Taking the Fourier transform of (15.18) converts the convolution into a product, and we can then solve for $\hat{b}_{z, \zeta}^{(2)}(k)$ to obtain

$$
\hat{b}_{z, \zeta}^{(2)}(k)=\frac{b_{z}^{(1)}}{1-\zeta b_{z}^{(1)} \hat{D}(k)} .
$$

Note that the denominator is zero when $z=\zeta=1$ and $k=0$.

Finally, we observe that the Fourier transform of (15.16) is given by

$$
\hat{b}_{z, \zeta}^{(2)}(k)=\sum_{(T, \varphi)} \sum_{j \in T} \mathbb{P}(T, \varphi) z^{|T|} \mathrm{e}^{\mathrm{i} k \cdot \varphi(j)} \zeta^{|j|} .
$$

We conclude from this and (15.15) that 


$$
\hat{b}_{1,1}^{(2)}(0)=\sum_{(T, \varphi)} \sum_{j \in T} \mathbb{P}(T, \varphi)=\sum_{n=1}^{\infty} n \mathbb{P}(|T|=n)=\infty .
$$

The two-point function given in Theorem 15.2 can be interpreted as the two-point function of simple random walk with an activity $\zeta$ associated to each step of the walk and an activity $b_{z}^{(1)}$ associated to each vertex. We may therefore regard a critical Poisson branching random walk configuration containing 0 and $x$ as corresponding to a simple random walk path from 0 to $x$ with a one-point function attached at each vertex along the way. This was the philosophy of the mean-field model of Section 15.1.

Next, we define the $r$-point functions for $r \geq 3$. Our definition keeps track of a substantial amount of information, and requires as preparation the following definitions of shape, subshape, skeleton and compatibility.

Shape: Shapes are certain rooted binary trees. For $r \geq 2$, we give a recursive definition of the set $\Sigma_{r}$ of $r$-shapes, as follows. Each $r$-shape has $2 r-3$ edges, $r-2$ vertices of degree 3 (the branch points) and $r$ vertices of degree 1 (the leaves) labelled $0,1, \ldots, r-1$. There is a unique 2 -shape given by the tree consisting of vertex 0 joined by a single edge to vertex 1 . We think of this shape as indicating that vertex 0 is an ancestor of vertex 1 . There is a unique 3 -shape, consisting of three vertices $0,1,2$ each joined by an edge to a fourth vertex. We think of this shape as indicating that 0 is an ancestor of both 1 and 2. In general, for $r \geq 3$, to each $(r-1)$-shape $\sigma$, we obtain $2 r-5 r$-shapes by choosing one of the $2 r-5$ edges of $\sigma$, adding a vertex on that edge together with a new edge that joins the added vertex to a new leaf $r-1$. The resulting $r$-shapes represent the different ways in which an additional $r^{\text {th }}$ particle can be added to the family tree of $r-1$ particles represented by $\sigma$. Thus there is a unique shape for $r=2$ and $r=3$, and $(2 r-5) ! !$ distinct shapes for $r \geq 4$, where we use the notation $(-1) ! !=1$, and, for $r \geq 3$,

$$
(2 r-5) ! !=\prod_{j=3}^{r}(2 j-5) .
$$

When $r$ is clear from the context, we will refer to an $r$-shape simply as a shape. For notational convenience, we associate to each shape an arbitrary labelling of its $2 r-3$ edges, with labels $1, \ldots, 2 r-3$. This arbitrary choice of edge labels is fixed once and for all; see Fig. 15.1.

Subshape: A subshape of a shape $\sigma \in \Sigma_{r}$ is a tree obtained by contracting a subset of the edges of $\sigma$ to a point. This can lead to multiply-labelled vertices, and contracted edges lose their labels. The subshapes for $r=3$ are shown in Fig. 15.2. In general, there are $2^{2 r-3}$ subshapes of a shape $\sigma \in \Sigma_{r}$. We denote subshapes by $\lambda$ and write $\lambda \leq \sigma$ when $\lambda$ is a subshape of $\sigma$. We denote the set of edge labels of $\lambda$ by $e(\lambda)$. 
158

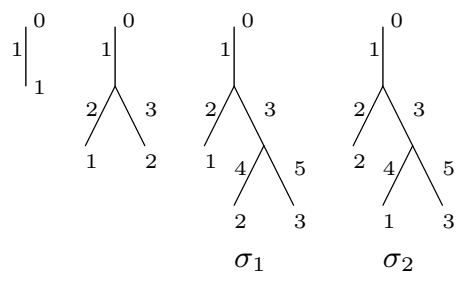

The Lace Expansion and its Applications

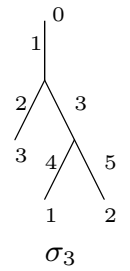

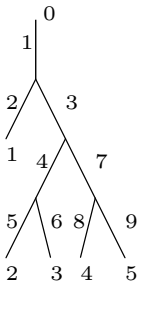

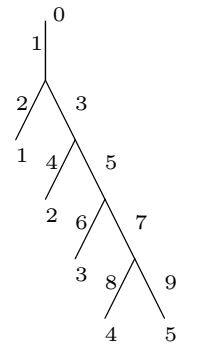

Fig. 15.1. The shapes for $r=2,3,4$, and examples of the $7 ! !=7 \cdot 5 \cdot 3=105$ shapes for $r=6$. The shapes' edge labels are arbitrary but fixed.

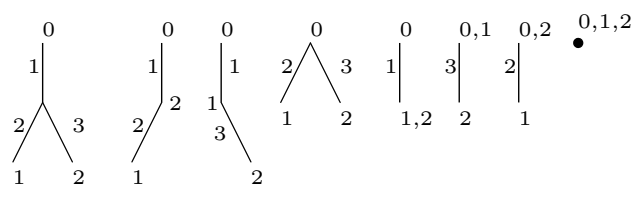

Fig. 15.2. The $2^{3}=8$ subshapes for $r=3$.

Skeleton: We write $\bar{\imath}=\left(i_{1}, \ldots, i_{r-1}\right)$ for a sequence of $r-1$ vertices $i_{j}$ (not necessarily distinct) in a tree $T$, and define the skeleton $B$ of $(T, \bar{\imath})$ to be the subtree of $T$ spanning $0, i_{1}, \ldots, i_{r-1}$. We will distinguish $r-1$ and $2 r-3$ component vectors by using $\vec{\cdot}$ and $\vec{\cdot}$, respectively.

Let $\beta_{B}$ denote the tree obtained from $B$ by ignoring vertices of degree 2 in $B$ other than $0, i_{1}, \ldots, i_{r-1}$ (which may have degree 2 in $B$ ), and by assigning label $j$ to vertex $i_{j}$ for each $j$. This may lead to multiple labels at a vertex, as is the case for subshapes.

Given $\sigma \in \Sigma_{r}$ and a subshape $\lambda \leq \sigma$, we say that $\beta_{B}$ is isomorphic to $\lambda$ if there is an edge preserving bijection from the set of all vertices of $\beta_{B}$ to the set of all vertices of $\lambda$, which preserves the vertex labels of $\beta_{B}$ and $\lambda$ (including any multiple labels at vertices). Given such an isomorphism, the edge labels of $\lambda$ induce labels on the edges of $\beta_{B}$ and thus on the paths in $T$ comprising the skeleton $B$.

Compatibility: Let $\sigma \in \Sigma_{r}, \vec{m}=\left(m_{1}, \ldots, m_{2 r-3}\right)$ for non-negative integers $m_{j}$, and $\vec{y}=\left(y_{1}, \ldots, y_{2 r-3}\right)$ for $y_{j} \in \mathbb{Z}^{d}$. Given $(T, \varphi)$, fix $r-1$ vertices $\bar{\imath}$ in $T$. We say that $(T, \varphi, \bar{\imath})$ is compatible with $(\sigma ; \vec{y}, \vec{m})$ if the following hold:

1. $\beta_{B}$ is isomorphic to a subshape $\lambda$ of $\sigma$ (in which case the paths of the skeleton $B$ have an induced labelling).

2. Let $l_{j}>0$ denote the length of the skeleton path labelled $j$, and let $l_{j}=0$ for any edge in $\sigma$ that is not in $\lambda$. Then $l_{j}=m_{j}$ for each $j=1, \ldots, 2 r-3$.

3. The image under $\varphi$ of the skeleton path (oriented away from the root) labelled $j$ undergoes the displacement $y_{j}$ for each $j$ labelling an edge in $\lambda$, and $y_{j}=0$ for any edge $j$ in $\sigma$ that is not in $\lambda$. 

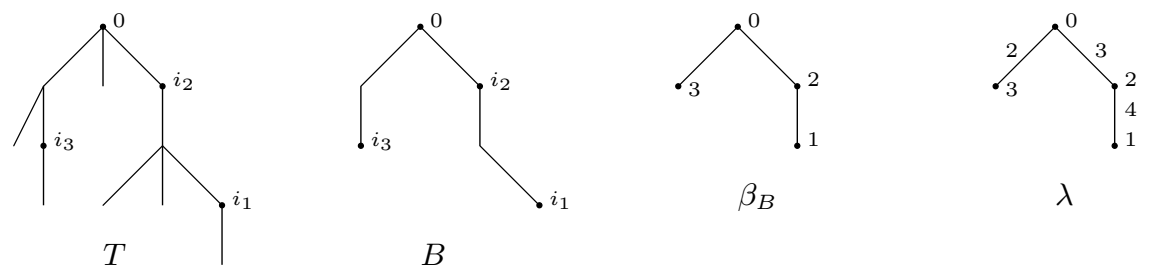

Fig. 15.3. A tree $T$ containing $i_{1}, i_{2}, i_{3}$, its skeleton $B$, the reduced skeleton $\beta_{B}$, and the subshape $\lambda$ of $\sigma_{3}$ (see Fig. 15.1) to which $\beta_{B}$ is isomorphic.

For example, given $(T, \bar{\imath})$ of Fig. 15.3, and any embedding $\varphi$ of $T,(T, \varphi, \bar{\imath})$ is compatible with $\left(\sigma_{3} ;\left(0, \varphi\left(i_{3}\right), \varphi\left(i_{2}\right), \varphi\left(i_{1}\right)-\varphi\left(i_{2}\right), 0\right),(0,2,1,2,0)\right)$.

The r-point functions: Let $r \geq 2, \sigma \in \Sigma_{r}, \vec{y}=\left(y_{1}, \ldots, y_{2 r-3}\right)$ with each $y_{i} \in \mathbb{Z}^{d}$, and let $\vec{m}=\left(m_{1}, \ldots, m_{2 r-3}\right)$ with each $m_{i}$ a non-negative integer. We define

$$
\begin{aligned}
& b_{n}^{(r)}(\sigma ; \vec{y}, \vec{m}) \\
& =\sum_{(T, \varphi):|T|=n} \mathbb{P}(T, \varphi) \sum_{i_{1}, \ldots, i_{r-1} \in T} I[(T, \varphi, \bar{\imath}) \text { is compatible with }(\sigma ; \vec{y}, \vec{m})] .
\end{aligned}
$$

Then we define the $r$-point function by

$$
b_{z, \vec{\zeta}}^{(r)}(\sigma ; \vec{y})=\sum_{n=0}^{\infty} \sum_{m_{1}, \ldots, m_{2 r-3}=0}^{\infty} b_{n}^{(r)}(\sigma ; \vec{y}, \vec{m}) z^{n} \prod_{j=1}^{2 r-3} \zeta_{j}^{m_{j}} .
$$

Exercise 15.3. Show that (15.24) agrees with the definition (15.16) for $r=2$.

The next theorem gives the Fourier transform of the $r$-point functions, where

$$
\hat{f}(\vec{k})=\sum_{y_{1}, \ldots, y_{2 r-3} \in \mathbb{Z}^{d}} f(\vec{y}) \mathrm{e}^{\mathrm{i} \vec{k} \cdot \vec{y}}
$$

with $\vec{k} \cdot \vec{y}=\sum_{j=1}^{2 r-3} k_{j} \cdot y_{j}$.

Theorem 15.4. For $d \geq 1, r \geq 2, \sigma \in \Sigma_{r}, k_{j} \in[-\pi, \pi]^{d},|z|<1,\left|\zeta_{j}\right| \leq 1$,

$$
\hat{b}_{z, \vec{\zeta}}^{(r)}(\sigma ; \vec{k})=\left(b_{z}^{(1)}\right)^{-2(r-2)} \prod_{j=1}^{2 r-3} \hat{b}_{z, \zeta_{j}}^{(2)}\left(k_{j}\right) .
$$

Before proving the theorem, we remark that the factor $\left(b_{z}^{(1)}\right)^{-2(r-2)}$ has a combinatorial interpretation. Namely, it "corrects" for an overcounting of the branch at each of the $r-2$ shape vertices of degree 3 , as this branch is counted 
in $\prod_{j=1}^{2 r-3} \hat{b}_{z, \zeta_{j}}^{(2)}\left(k_{j}\right)$ once by each of the three two-point functions incident at that vertex. This factor is equal to 1 at the critical point $z=1$, and does not play a role in the leading critical behaviour.

Proof of Theorem 15.4. The statement of the theorem is a tautology for $r=2$, so we consider $r \geq 3$. Let

$$
\hat{q}_{z, \zeta}^{(2)}(k)=\zeta \hat{D}(k) \hat{b}_{z, \zeta}^{(2)}(k)
$$

By (15.18), $\hat{b}_{z, \zeta}^{(2)}(k)=b_{z}^{(1)}\left[1+\hat{q}_{z, \zeta}^{(2)}(k)\right]$, so it suffices to show that

$$
\hat{b}_{z, \vec{\zeta}}^{(r)}(\sigma ; \vec{k})=b_{z}^{(1)} \prod_{j=1}^{2 r-3}\left(1+\hat{q}_{z, \zeta_{j}}^{(2)}\left(k_{j}\right)\right) .
$$

Expanding the product, the desired identity (15.28) is equivalent to

$$
\hat{b}_{z, \vec{\zeta}}^{(r)}(\sigma ; \vec{k})=b_{z}^{(1)} \sum_{\lambda \leq \sigma} \prod_{j \in e(\lambda)} \hat{q}_{z, \zeta_{j}}^{(2)}\left(k_{j}\right)
$$

Given a subshape $\lambda \leq \sigma$, we let $b(\lambda)$ denote the result of restricting the summation in (15.24) to $m_{j}=0$ (and thus $y_{j}=0$ ) for $j \notin e(\lambda)$ and $m_{j}>0$ for $j \in e(\lambda)$. Its Fourier transform will be denoted $\hat{b}(\lambda)$. We leave implicit the dependence on the variables of $\vec{k}$ and $\vec{\zeta}$, as these are determined by the edge labels of $\lambda$. Then

$$
\hat{b}_{z, \vec{\zeta}}^{(r)}(\sigma ; \vec{k})=\sum_{\lambda \leq \sigma} \hat{b}(\lambda)
$$

Thus it suffices to show that

$$
\hat{b}(\lambda)=b_{z}^{(1)} \prod_{j \in e(\lambda)} \hat{q}_{z, \zeta_{j}}^{(2)}\left(k_{j}\right) .
$$

This is clear if $\lambda$ consists of a single vertex, so it suffices to consider the case where $\lambda$ contains at least one edge.

We use $\pi$ to denote a subshape for which the root has degree 1 , and write $\hat{q}(\pi)=\left(z p_{1}\right)^{-1} \hat{b}(\pi)$. The factor $\left(z p_{1}\right)^{-1}$ serves to cancel the factor $z p_{1}=z \mathrm{e}^{-1}$ associated to the root in $\hat{b}(\pi)$. Given a subshape $\lambda$ having at least one edge, let $\pi_{1}, \ldots, \pi_{l}$ be the branches emerging from its root. As in (15.18), using now that the $l^{\text {th }}$ derivative of $P$ obeys $z P^{(l)}\left(b_{z}^{(1)}\right)=b_{z}^{(1)}$,

$$
\begin{aligned}
\hat{b}(\lambda) & =\sum_{j=l}^{\infty} z p_{j} j(j-1) \cdots(j-l+1)\left(b_{z}^{(1)}\right)^{j-l} \prod_{a=1}^{l} \hat{q}\left(\pi_{a}\right) \\
& =b_{z}^{(1)} \prod_{a=1}^{l} \hat{q}\left(\pi_{a}\right) .
\end{aligned}
$$


Let $\bar{\pi}$ denote the subshape obtained from $\pi$ by contracting the edge incident to the root. We claim that

$$
\hat{q}(\pi)=\hat{q}_{z, \zeta}^{(2)}(k) \frac{1}{b_{z}^{(1)}} \hat{b}(\bar{\pi}),
$$

where $\zeta$ and $k$ bear the subscript of the label of the edge incident on the root of $\pi$. From this, the desired result (15.31) then follows by substituting (15.33) into (15.32) recursively.

To prove (15.33), we condition on whether the length of the tree's skeleton path, corresponding to the edge of $\pi$ incident on the root, is equal to or greater than 1 . This leads, by conditioning as in (15.18), to

$$
\hat{q}(\pi)=\zeta \hat{D}(k) \hat{b}(\bar{\pi})+\zeta \hat{D}(k) b_{z}^{(1)} \hat{q}(\pi) .
$$

Solving and using (15.17) and (15.27), we obtain

$$
\hat{q}(\pi)=\frac{\zeta \hat{D}(k)}{1-\zeta \hat{D}(k) b_{z}^{(1)}} \hat{b}(\bar{\pi})=\hat{q}_{z, \zeta}^{(2)}(k) \frac{1}{b_{z}^{(1)}} \hat{b}(\bar{\pi}),
$$

which is (15.33).

\subsection{Weakly Self-Avoiding Lattice Trees}

The weakly self-avoiding walk has played an important role in the development of the theory of self-avoiding walks. There has been no parallel situation for lattice trees, perhaps because it is less obvious how to define weakly selfavoiding lattice trees. In this section we give a natural definition of weakly self-avoiding lattice trees and prove that it corresponds to usual lattice trees in the limit of infinite self-avoidance strength. Throughout the section, we follow the presentation of [30]. Presumably weakly self-avoiding lattice trees are in the same universality class as usual lattice trees, no matter how weak the self-avoidance.

Let $\mathbb{P}(T, \varphi)$ be given by (15.7)-(15.8). Given $\beta \geq 0$, let

$$
Z_{n}^{\beta}=\sum_{(T, \varphi):|T|=n} \mathbb{P}(T, \varphi) \exp \left[-\frac{1}{2} \beta \sum_{i, j \in T: i \neq j} I[\varphi(i)=\varphi(j)]\right],
$$

and, for $|T|=n$, define

$$
\mathbb{Q}_{n}^{\beta}(T, \varphi)=\frac{1}{Z_{n}^{\beta}} \mathbb{P}(T, \varphi) \exp \left[-\frac{1}{2} \beta \sum_{i, j \in T: i \neq j} I[\varphi(i)=\varphi(j)]\right] .
$$

The measure $\mathbb{Q}_{n}^{\beta}$ on the set of $n$-vertex branching random walk configurations rewards self-avoidance by giving a penalty $\mathrm{e}^{-\beta}$ to each self-intersection. For $\beta=0, \mathbb{Q}_{n}^{0}$ is just branching random walk conditional on $|T|=n$. The next theorem shows that the weakly self-avoiding lattice trees interpolate between 
branching random walk and lattice trees, in the sense that $\mathbb{Q}_{n}^{\infty}$ corresponds in an appropriate sense to the uniform measure on the set of $n$-vertex lattice trees containing the origin.

In the statement of the theorem $t_{n}^{(1)}$ denotes the number of $n$-vertex lattice trees containing the origin, as in Section 7.1. Given an injective $\varphi$ and a lattice tree $L$, we abuse notation by writing $\varphi(T)=L$ if $\varphi(T)$ consists of the vertices in $L$ and the edges in $T$ are mapped to the bonds in $L$.

Theorem 15.5. For $d \geq 1$ and $n \geq 1$,

$$
\lim _{\beta \rightarrow \infty} \mathbb{Q}_{n}^{\beta}(T, \varphi)=0
$$

if $\varphi$ is not injective. On the other hand, given an n-vertex lattice tree $L$ containing the origin,

$$
\lim _{\beta \rightarrow \infty} \sum_{(T, \varphi): \varphi(T)=L} \mathbb{Q}_{n}^{\beta}(T, \varphi)=\frac{1}{t_{n}^{(1)}} .
$$

Proof. The first statement of the theorem, for non-injective $\varphi$, follows immediately from the definition of $\mathbb{Q}_{n}^{\beta}$.

For the second statement of the theorem, let $\mathcal{T}_{n}$ denote the set of $n$-vertex lattice trees containing the origin. This has cardinality $t_{n}^{(1)}$. We will prove that

$$
\sum_{(T, \varphi): \varphi(T)=L} \mathbb{P}(T, \varphi)=(2 d)^{-(n-1)} \mathrm{e}^{-n}
$$

for every $L \in \mathcal{T}_{n}$. The important point for the proof is that the right hand side is the same for all $L \in \mathcal{T}_{n}$, and its particular value plays no role. In fact, given (15.40), we then have

$$
Z_{n}^{\infty}=\sum_{L \in \mathcal{T}_{n}} \sum_{(T, \varphi): \varphi(T)=L} \mathbb{P}(T, \varphi)=t_{n}^{(1)}(2 d)^{-(n-1)} \mathrm{e}^{-n},
$$

which gives the desired result that

$$
\sum_{(T, \varphi): \varphi(T)=L} \mathbb{Q}_{n}^{\infty}(T, \varphi)=\frac{1}{Z_{n}^{\infty}} \sum_{(T, \varphi): \varphi(T)=L} \mathbb{P}(T, \varphi)=\frac{1}{t_{n}^{(1)}} .
$$

To prove (15.40), we first note that by (15.7) and (15.8),

$$
\sum_{(T, \varphi): \varphi(T)=L} \mathbb{P}(T, \varphi)=(2 d)^{-(n-1)} \mathrm{e}^{-n} \sum_{(T, \varphi): \varphi(T)=L} \prod_{i \in T} \frac{1}{\xi_{i}},
$$

where $\xi_{i}$ is the number of offspring of vertex $i$. It suffices to show that

$$
\sum_{(T, \varphi): \varphi(T)=L} \prod_{i \in T} \frac{1}{\xi_{i} !}=1
$$


Let $b_{0}$ be the degree of 0 in $L$, and given nonzero $x \in L$, let $b_{x}$ be the degree of $x$ in $L$ minus 1 (the forward degree of $x$ ). Then the set $\left\{b_{x}: x \in L\right\}$ (with repetitions) must be equal to the set of $\xi_{i}$ (with repetitions) for any $T$ that can be mapped to $L$. Defining $\nu(L)$ to be the cardinality of $\{(T, \varphi): \varphi(T)=L\}$, (15.44) is therefore equivalent to

$$
\nu(L)=\prod_{x \in L} b_{x} !
$$

We prove (15.45) by induction on the number $N$ of generations of $L$. By this, we mean the length of the longest self-avoiding path in $L$, starting from the origin. The identity (15.45) clearly holds if $N=0$. Our induction hypothesis is that (15.45) holds if there are $N-1$ or fewer generations. Suppose $L$ has $N$ generations, and let $L_{1}, \ldots, L_{b_{0}}$ denote the lattice trees resulting from deleting from $L$ the origin and all bonds incident on the origin. We regard each $L_{a}$ as rooted at the neighbour of the origin in the corresponding deleted bond. It suffices to show that $\nu(L)=b_{0} ! \prod_{a=1}^{b_{0}} \nu\left(L_{a}\right)$, since each $L_{a}$ has fewer than $N$ generations.

To prove this, we note that each pair $(T, \varphi)$ with $\varphi(T)=L$ induces a set of $\left(T_{a}, \varphi_{a}\right)$ such that $\varphi_{a}\left(T_{a}\right)=L_{a}$. This correspondence is $b_{0}$ ! to 1 , since $(T, \varphi)$ is determined by the set of $\left(T_{a}, \varphi_{a}\right)$, up to permutation of the branches of $T$ at its root. See Exercise 15.6. This proves $\nu(L)=b_{0} ! \prod_{a=1}^{b_{0}} \nu\left(L_{a}\right)$.

Exercise 15.6. Let $L$ be the 2-dimensional lattice tree consisting of bonds $\left\{0, e_{2}\right\},\left\{0,-e_{2}\right\},\left\{0, e_{1}\right\},\left\{e_{1}, 2 e_{1}\right\},\left\{e_{1}, e_{1}+e_{2}\right\}$, where $e_{1}=(1,0)$ and $e_{2}=$ $(0,1)$. Let $T_{1}$ be the single vertex $0_{1}$ and $T_{2}$ be the single vertex $0_{2}$, with $\varphi_{1}\left(0_{1}\right)=e_{2}$ and $\varphi_{2}\left(0_{2}\right)=-e_{2}$. Let $T_{3}$ be the tree consisting of the root 0 and its two offspring 00 and 01 , with $\varphi_{3}(0)=e_{1}, \varphi_{3}(00)=2 e_{1}, \varphi_{3}(01)=e_{1}+e_{2}$. Write down the six distinct $(T, \varphi)$ that correspond to the collection $\left(T_{a}, \varphi_{a}\right)$ $(a=1,2,3)$ as in the last paragraph of the proof of Theorem 15.5.

\section{Integrated Super-Brownian Excursion}

In this section, we discuss integrated super-Brownian excursion (ISE), and describe how it arises as a scaling limit of branching random walk in all dimensions, and of lattice trees and critical percolation clusters above their upper critical dimensions.

To formulate a scaling limit of branching random walk, it is necessary to decide how much information the limit should keep track of. One possibility, discussed in this section, is to condition the branching random walk to have a total of $n$ particles (counting multiple occupations of a vertex and including all generations), to assign mass $n^{-1}$ to each vertex, and to take the scaling limit of this random mass distribution. The mass distribution due to a configuration of branching random walk is a random discrete probability measure on $\mathbb{R}^{d}$, and we are led naturally to study random probability measures on $\mathbb{R}^{d}$, which is to 
say that we study probability measures on probability measures. The scaling limit in this framework is ISE. Much of the theory of ISE was developed in $[14,15,151]$. Our analysis will be based on explicit formulas for the moment measures of ISE, which can be found in $[15,152]$. The material in this section is based on [30, 61, 105, 188].

The above description of a branching random walk configuration as a mass distribution ignores issues of time evolution and genealogy. As we will see, information about both of these can be recovered, but the focus in this section is primarily on mass distribution alone. We will return to the other issues in Section 17, where the canonical measure of super-Brownian motion will be discussed.

\subsection{Moment Measures of Branching Random Walk}

Let $M_{1}\left(\mathbb{R}^{d}\right)$ denote the set of Borel probability measures on $\mathbb{R}^{d}$, equipped with the topology of weak convergence. Borel sets in $M_{1}\left(\mathbb{R}^{d}\right)$ can be defined using this topology, and we consider Borel measures on $M_{1}\left(\mathbb{R}^{d}\right)$.

Branching random walk configurations $(T, \varphi)$ with $|T|=n$ induce a measure $\mu_{n}$ on $M_{1}\left(\mathbb{R}^{d}\right)$, as follows. Let $\delta_{y}$ be the point mass at $y \in \mathbb{R}^{d}$, i.e., $\delta_{y}(A)=1$ if the Borel set $A$ contains $y$, and otherwise $\delta_{y}(A)=0$. Given a tree $T$ with $|T|=n$, and an embedding $\varphi$ of $T$, let

$$
\mu(T, \varphi)=\frac{1}{n} \sum_{i \in T} \delta_{n^{-1 / 4} \varphi(i)}
$$

Thus $\mu(T, \varphi)$ is the probability measure on $\mathbb{R}^{d}$ which assigns mass $\frac{1}{n}$ to $n^{-1 / 4} \varphi(i)$ for each $i \in T$. We then define the measure $\mu_{n}$ on $M_{1}\left(\mathbb{R}^{d}\right)$ to be the measure that assigns mass $\mathbb{P}((T, \varphi)|| T \mid=n)$ to each $\mu(T, \varphi)$ with $|T|=n$. In other words, we obtain a random probability measure on $\mathbb{R}^{d}$ by assigning equal mass to each of the $n$ embedded vertices of a rescaled version of an embedded tree.

We may then ask if the measures $\mu_{n}$ converge weakly to some measure $\mu_{\mathrm{ISE}}$ on $M_{1}\left(\mathbb{R}^{d}\right)$. Here, weak convergence (see [24]) is the assertion that for any real-valued bounded continuous function $F$ on $M_{1}\left(\mathbb{R}^{d}\right)$,

$$
\lim _{n \rightarrow \infty} \int_{M_{1}\left(\mathbb{R}^{d}\right)} F(\nu) \mathrm{d} \mu_{n}(\nu)=\int_{M_{1}\left(\mathbb{R}^{d}\right)} F(\nu) \mathrm{d} \mu_{\mathrm{ISE}}(\nu) .
$$

A useful ingredient in answering this question is to study the convergence of the moment measures of $\mu_{n}$. The $l^{\text {th }}$ moment measure $M^{(l)}$ of a probability measure $\mu$ on $M_{1}\left(\mathbb{R}^{d}\right)$ is the deterministic probability measure on $\left(\mathbb{R}^{d}\right)^{l}$ defined, for $l \geq 1$, by

$$
d M^{(l)}\left(x_{1}, \ldots, x_{l}\right)=\int_{M_{1}\left(\mathbb{R}^{d}\right)} \mathrm{d} \mu(\nu) \mathrm{d} \nu\left(x_{1}\right) \cdots \mathrm{d} \nu\left(x_{l}\right) .
$$


To prove weak convergence of the moment measures, it is sufficient to prove pointwise convergence of their characteristic functions.

To write down the moment measures for $\mu_{n}$, for $l \geq 1$ we define

$$
s_{n}^{(l+1)}(\bar{x})=\sum_{(T, \varphi):|T|=n} \mathbb{P}(T, \varphi) \sum_{i_{1}, \ldots, i_{l} \in T} \prod_{j=1}^{l} I\left[\varphi\left(i_{j}\right)=x_{j}\right],
$$

where $\bar{x}=\left(x_{1}, \ldots, x_{l}\right)$. Note that summation of (16.4) over $\bar{x} \in \mathbb{R}^{d l}$ gives $n^{l} \mathbb{P}(|T|=n)$. Interpreting (16.3) in this context, the $l^{\text {th }}$ moment measure $M_{n}^{(l)}$ of $\mu_{n}$ is the probability measure on $\mathbb{R}^{d l}$ which places mass equal to $\left[n^{l} \mathbb{P}(|T|=n)\right]^{-1} s_{n}^{(l+1)}(\bar{x})$ at $n^{-1 / 4} \bar{x}$, for $\bar{x} \in \mathbb{Z}^{d l}$. The characteristic function $\hat{M}_{n}^{(l)}(\bar{k})$ of $M_{n}^{(l)}$ is therefore given by

$$
\hat{M}_{n}^{(l)}(\bar{k})=\frac{1}{n^{l} \mathbb{P}(|T|=n)} \hat{s}_{n}^{(l+1)}\left(\bar{k} n^{-1 / 4}\right),
$$

where

$$
\hat{f}(\bar{k})=\sum_{\bar{x}} f(\bar{x}) \mathrm{e}^{\mathrm{i} \bar{k} \cdot \bar{x}}
$$

with $\bar{k} \cdot \bar{x}=\sum_{j=1}^{l} k_{j} \cdot x_{j}$. We wish to compute the limit of $\hat{M}_{n}^{(l)}(\bar{k})$ as $n \rightarrow \infty$. The asymptotic behaviour of the denominator of (16.5) is given by (15.15) as $(2 \pi)^{-1 / 2} n^{l-3 / 2}$, so we are left with the numerator.

The limiting behaviour of the numerator will be discussed in Section 16.3. It seems clear that there should be a close relationship between the coefficients $\hat{b}_{n}^{(r)}(\sigma ; \vec{k}, \vec{m})$ of $(15.23)-(15.24)$ and $\hat{s}_{n}^{(r)}(\bar{k})$. In fact, $b_{n}^{(r)}(\sigma ; \vec{y}, \vec{m})$ contains more information than $s_{n}^{(r)}(\bar{x})$, as it specifies the skeleton displacements in addition to $\bar{x}$. So we first consider the asymptotic behaviour of $\hat{b}_{n}^{(r)}(\sigma ; \vec{k}, \vec{m})$. Since we know the generating functions $\hat{b}_{z, \vec{\zeta}}^{(r)}(\sigma ; \vec{k})$ from Section 15.2, this will be studied within the framework of the question: If we know the generating function of a sequence, what can we say about the asymptotic behaviour of the sequence itself? The rule of thumb is that the latter is determined by the singularity of the generating function that is closest to the origin. Moreover, the nature of the singularity can be described in terms of critical exponents.

\subsection{Critical Exponents and Generating Functions}

In this section, we determine the asymptotic behaviour of $\hat{b}_{n}^{(r)}(\sigma ; \vec{k}, \vec{m})$. To do so, we first study the singularity structure of the generating function $\hat{b}_{z, \vec{\zeta}}^{(r)}(\sigma ; \vec{k})$, beginning with $r=2$. This holds the key to the study of $r \geq 3$, by Theorem 15.4 .

The generating function $\hat{b}_{z, \zeta}^{(2)}(k)$ is given by $(15.17)$, in terms of the onepoint function $b_{z}^{(1)}$ of Theorem 15.1. Properties of the one-point function can 
be derived from known properties of the Lambert function, or derived directly from (15.12). In particular, $b_{z}^{(1)}$ has a square root singularity at $z=1$, and

$$
b_{z}^{(1)}=1-\sqrt{2}(1-z)^{1 / 2}+O(|1-z|),
$$

with the absolute value of the error term bounded by a constant multiple of $|1-z|$ uniformly in the cut plane $\mathbb{C} \backslash[1, \infty)$. The square root is the branch with $(1-z)^{1 / 2}>0$ for $z<1$.

Exercise 16.1. Prove (16.7).

Using also the fact that $\hat{D}(k)=1-(2 d)^{-1} k^{2}+O\left(k^{4}\right)$ by (1.12), it follows from (15.17) that

$$
\hat{b}_{z, \zeta}^{(2)}(k)=\frac{1+E_{1}}{(2 d)^{-1}|k|^{2}+2^{1 / 2} \sqrt{1-z}+(1-\zeta)+E_{2}},
$$

where $E_{1}=-\sqrt{2}(1-z)^{1 / 2}+O(|1-z|)$ is small compared to 1 for $z$ near 1 , and where $E_{2}$ is a sum of terms of order $|k|^{4},|k|^{2}(1-\zeta),|1-z|$, and similar terms which are higher order than $|k|^{2}, \sqrt{1-z}$ and $(1-\zeta)$.

The formula (16.8) has significance for the values of the critical exponents of branching random walk. Namely, setting $k=0$ and $\zeta=1$ in (16.8), we see that $\hat{b}_{z, 1}^{(2)}(0) \sim 2^{-1 / 2}(1-z)^{-1 / 2}$, and hence the critical exponent $\gamma$ for the susceptibility of branching random walk is $\gamma=\frac{1}{2}$. Setting $z=1$ and $\zeta=1$ in (16.8), we see that $\hat{b}_{1,1}^{(2)}(k) \sim 2 d|k|^{-2}$, and hence the critical exponent $\eta$ for the critical two-point function of branching random walk is $\eta=0$. Finally, setting $k=0$ and $z=1$ gives $\hat{b}_{1, \zeta}^{(2)}(0) \sim(1-\zeta)^{-1}$ (this is, in fact, an equality by (15.17)), so the "backbone exponent" is equal to 1 . In addition, information is provided by (16.8) concerning the additive form in the denominator for the joint behaviour as $k \rightarrow 0, z \rightarrow 1$, and $\zeta \rightarrow 1$.

The error terms $E_{1}$ and $E_{2}$ in (16.8) need to be taken into account in an analysis of the moment measures of branching random walk, but this is merely a nuisance and they do not add anything meaningful. In the following, we will simplify the problem by temporarily setting these error terms equal to zero, obtaining

$$
\hat{b}_{z, \zeta}^{(2)}(k) \approx \frac{1}{(2 d)^{-1}|k|^{2}+2^{1 / 2} \sqrt{1-z}+(1-\zeta)} .
$$

Thus, for our simplified version of the two-point function, for $k \in \mathbb{R}^{d}$ and $\zeta, z \in \mathbb{C}$ with $|\zeta|,|z|<1$, we define $H_{z, \zeta}^{(2)}(k)$ by

$$
H_{z, \zeta}^{(2)}(k)=\frac{1}{(2 d)^{-1}|k|^{2}+2^{1 / 2} \sqrt{1-z}+(1-\zeta)},
$$

where the square root has branch cut $[1, \infty)$ and is positive for real $z<1$. In addition, for $r \geq 2, k_{j} \in \mathbb{R}^{d}$ and $\zeta_{j} \in \mathbb{C}$, with $\left|\zeta_{j}\right|<1$, we write $\vec{k}=$ $\left(k_{1}, \ldots, k_{2 r-3}\right)$ and $\vec{\zeta}=\left(\zeta_{1}, \ldots, \zeta_{2 r-3}\right)$, and define 


$$
H_{z, \vec{\zeta}}^{(r)}(\vec{k})=\prod_{j=1}^{2 r-3} H_{z, \zeta_{j}}^{(2)}\left(k_{j}\right)
$$

analogous to (15.26). We write the coefficients in the Taylor expansion of (16.11) as

$$
H_{z, \vec{\zeta}}^{(r)}(\vec{k})=\sum_{m_{1}, \ldots, m_{2 r-3}=0}^{\infty} \sum_{n=0}^{\infty} h_{n, \vec{m}}^{(r)}(\vec{k}) z^{n} \prod_{j=1}^{2 r-3} \zeta_{j}^{m_{j}}
$$

We write $g_{\vec{m}}^{(r)}(\vec{k})=\sum_{n=0}^{\infty} h_{n, \vec{m}}^{(r)}(\vec{k})$ for the coefficient of $\prod_{j=1}^{2 r-3} \zeta_{j}^{m_{j}}$ in $H_{1, \vec{\zeta}}^{(r)}(\vec{k})$. Writing $\overrightarrow{1}=(1, \ldots, 1)$, we denote the coefficient of $z^{n}$ in $H_{z, \overrightarrow{1}}^{(r)}(\vec{k})$ by

$$
h_{n}^{(r)}(\vec{k})=\sum_{m_{1}, \ldots, m_{2 r-3}=0}^{\infty} h_{n, \vec{m}}^{(r)}(\vec{k}) .
$$

To state the scaling limits of $h_{n}^{(r)}(\vec{k})$ and $h_{n, \vec{m}}^{(r)}(\vec{k})$, for $k_{i} \in \mathbb{R}^{d}$ and $t_{i} \geq 0$, we define

$$
\hat{a}^{(r)}(\vec{k}, \vec{t})=\left(\sum_{i=1}^{2 r-3} t_{i}\right) \mathrm{e}^{-\left(\sum_{i=1}^{2 r-3} t_{i}\right)^{2} / 2} \mathrm{e}^{-\sum_{i=1}^{2 r-3}\left|k_{i}\right|^{2} t_{i} / 2 d}
$$

and

$$
\hat{A}^{(r)}(\vec{k})=\int_{0}^{\infty} \mathrm{d} t_{1} \cdots \int_{0}^{\infty} \mathrm{d} t_{2 r-3} \hat{a}^{(r)}(\vec{k}, \vec{t}) .
$$

Explicitly, for $r=2$,

$$
\hat{A}^{(2)}(k)=\int_{0}^{\infty} t \mathrm{e}^{-t^{2} / 2} \mathrm{e}^{-|k|^{2} t / 2 d} \mathrm{~d} t .
$$

The integral (16.16) can be written in terms of the parabolic cylinder function $D_{-2}$ as $\hat{A}^{(2)}(\sqrt{d} k)=\mathrm{e}^{k^{4} / 16} D_{-2}\left(|k|^{2} / 2\right)[83,3.462 .1]$. The integrals $(16.15)$ obey

$$
\hat{A}^{(r)}(\overrightarrow{0})=\frac{1}{(2 r-5) ! !},
$$

where we recall the notation of (15.22), see Exercise 16.4.

We write $\lfloor\overrightarrow{t n}\rfloor$ to denote the vector with components $\left\lfloor t_{j} n\right\rfloor$.

Proposition 16.2. For $r \geq 2$, as $n \rightarrow \infty$,

$$
\begin{aligned}
g_{\lfloor\vec{t} n\rfloor}^{(r)}\left(\vec{k} n^{-1 / 2}\right) & \rightarrow \mathrm{e}^{-\sum_{j=1}^{2 r-3}\left|k_{j}\right|^{2} t_{j} / 2 d}, \\
h_{n}^{(r)}\left(\vec{k} n^{-1 / 4}\right) & \sim \frac{1}{\sqrt{2 \pi}} n^{r-5 / 2} \hat{A}^{(r)}(\vec{k}), \\
h_{n,\left\lfloor\vec{t} n^{1 / 2}\right\rfloor}^{(r)}\left(\vec{k} n^{-1 / 4}\right) & \sim \frac{1}{\sqrt{2 \pi}} \frac{1}{n} \hat{a}^{(r)}(\vec{k}, \vec{t}) .
\end{aligned}
$$


Proof. For (16.18), we use the fact that $H_{1, \zeta}^{(2)}(k)$ is the sum of a geometric series in $\zeta$, namely

$$
H_{1, \zeta}^{(2)}(k)=\frac{1}{(2 d)^{-1}|k|^{2}+(1-\zeta)}=\sum_{m=0}^{\infty} \frac{1}{\left(1+|k|^{2} / 2 d\right)^{m+1}} \zeta^{m} .
$$

Therefore $g_{\vec{m}}^{(r)}(\vec{k})=\prod_{j=1}^{2 r-3}\left(1+\left|k_{j}\right|^{2} / 2 d\right)^{-\left(m_{j}+1\right)}$, and (16.18) follows.

For the remainder of the proof, we follow [62], and to simplify the formulas we put $d=1$ (the general case then easily follows). For (16.19), it follows from the Cauchy integral formula that

$$
h_{n}^{(r)}\left(\vec{k} n^{-1 / 4}\right)=\frac{1}{2 \pi i} \oint \frac{d z}{z^{n+1}} \prod_{j=1}^{2 r-3} \frac{2}{\left|k_{j}\right|^{2} n^{-1 / 2}+2^{3 / 2}(1-z)^{1 / 2}},
$$

where the integral is taken around a small circle centred at the origin. For simplicity, in the proof of (16.19) we suppose that $k_{j} \neq 0$ for all $j$. See Exercise 16.3 for the general case. We make the change of variables $w=n(z-1)$, and then deform the contour to the branch cut $[0, \infty)$ in the $w$-plane. The resulting contour goes from right to left below the branch cut and from left to right above the cut. We then apply the identity

$$
\frac{2}{\left|k_{j}\right|^{2}+2^{3 / 2}(-w)^{1 / 2}}=\int_{0}^{\infty} \mathrm{d} t_{j} \mathrm{e}^{-\left|k_{j}\right|^{2} t_{j} / 2} \mathrm{e}^{-2^{1 / 2}(-w)^{1 / 2} t_{j}} .
$$

Taking into account the correct branch of the square root on either side of the branch cut, and applying Fubini's theorem, this gives

$$
\begin{aligned}
h_{n}^{(r)}\left(\vec{k} n^{-1 / 4}\right)=n^{r-5 / 2} \int_{0}^{\infty} \mathrm{d} t_{1} \cdots \int_{0}^{\infty} \mathrm{d} t_{2 r-3} \mathrm{e}^{-\left(\sum_{j=1}^{2 r-3}\left|k_{j}\right|^{2} t_{j}\right) / 2} \\
\quad \times \frac{1}{\pi} \int_{0}^{\infty} \frac{\mathrm{d} w}{(1+w / n)^{n+1}} \sin \left(\left(\sum_{j=1}^{2 r-3} t_{j}\right) \sqrt{2 w}\right) .
\end{aligned}
$$

Since $\left(1+\frac{w}{n}\right)^{n+1} \geq 1+\frac{(n+1) n}{2}\left(\frac{w}{n}\right)^{2} \geq 1+\frac{w^{2}}{2}$ for all $n \geq 1$, the dominated convergence theorem can be applied to conclude that as $n \rightarrow \infty$ the $w$-integral converges to

$$
\int_{0}^{\infty} \mathrm{d} w \mathrm{e}^{-w} \sin \left(\left(\sum_{j=1}^{2 r-3} t_{j}\right) \sqrt{2 w}\right)=\left(\frac{\pi}{2}\right)^{1 / 2}\left(\sum_{j=1}^{2 r-3} t_{j}\right) \mathrm{e}^{-\left(\sum_{j=1}^{2 r-3} t_{j}\right)^{2} / 2} .
$$

Therefore, as required,

$$
h_{n}^{(r)}\left(\vec{k} n^{-1 / 4}\right) \sim \frac{1}{\sqrt{2 \pi}} n^{r-5 / 2} \hat{A}^{(r)}(\vec{k}) .
$$

Next, we prove (16.20) (again taking $d=1$ for simplicity). Since each factor in the product in (16.11) is the sum of a geometric series in $\zeta_{j}$, 


$$
\begin{aligned}
& h_{n,\left\lfloor\vec{t} n^{1 / 2}\right\rfloor}^{(r)}\left(\vec{k} n^{-1 / 4}\right) \\
& \quad=\frac{1}{2 \pi i} \oint \frac{d z}{z^{n+1}} \prod_{j=1}^{2 r-3}\left(1+\frac{\left|k_{j}\right|^{2}}{2 \sqrt{n}}+\sqrt{2}(1-z)^{1 / 2}\right)^{-\left(\left\lfloor t_{j} n^{1 / 2}\right\rfloor+1\right)},
\end{aligned}
$$

where the integration is performed around a small circle centred at the origin. Making the change of variables $w=n(z-1)$ and deforming the contour of integration to the branch cut $[0, \infty)$ in the $w$-plane, the above is equal to

$$
\frac{1}{\pi n} \operatorname{Im} \int_{0}^{\infty} \frac{\mathrm{d} w}{(1+w / n)^{n+1}} \prod_{j=1}^{2 r-3}\left(1+\frac{\left|k_{j}\right|^{2}}{2 \sqrt{n}}-\frac{i \sqrt{2 w}}{\sqrt{n}}\right)^{-\left(\left\lfloor t_{j} n^{1 / 2}\right\rfloor+1\right)} .
$$

The integrand here is dominated by $\left(1+\frac{w^{2}}{2}\right)^{-1} \cdot 1$, so by the dominated convergence theorem, this is asymptotic to

$$
\frac{1}{\pi n} \mathrm{e}^{-\left(\sum_{j=1}^{2 r-3}\left|k_{j}\right|^{2} t_{j}\right) / 2} \int_{0}^{\infty} \mathrm{d} w \mathrm{e}^{-w} \sin \left(\left(\sum_{j=1}^{2 r-3} t_{j}\right) \sqrt{2 w}\right)=\frac{1}{\sqrt{2 \pi} n} \hat{a}^{(r)}(\vec{k}, \vec{t})
$$

using (16.25) in the last step.

Exercise 16.3. Prove (16.19) in the degenerate case where one or more $k_{j}$ is zero.

Exercise 16.4. Compute the asymptotic behaviour of $h_{n}^{(r)}(\overrightarrow{0})$ directly from its definition, and conclude (16.17) from (16.19).

By taking a little additional care with the error terms $E_{1}$ and $E_{2}$ in (16.8), a similar analysis shows that for $r \geq 2$, as $n \rightarrow \infty$,

$$
\begin{aligned}
\sum_{\vec{m}} \hat{b}_{n}^{(r)}\left(\sigma ; \vec{k} n^{-1 / 4}, \vec{m}\right) & \sim \frac{1}{\sqrt{2 \pi}} n^{r-5 / 2} \hat{A}^{(r)}(\vec{k}), \\
\hat{b}_{n}^{(r)}\left(\sigma ; \vec{k} n^{-1 / 4},\left\lfloor\vec{t} n^{1 / 2}\right\rfloor\right) & \sim \frac{1}{\sqrt{2 \pi}} \frac{1}{n} \hat{a}^{(r)}(\vec{k}, \vec{t}) .
\end{aligned}
$$

The implications of this for the scaling limit of branching random walk will be discussed in the next section. For now, we note that in (16.31) the scaling of time (distance along the skeleton) is $n^{1 / 2}$. Since space is being scaled as $n^{1 / 4}$, this is Brownian scaling. Thus (16.31) provides an interpretation of the variables $t_{j}$ in $\hat{a}^{(r)}(\vec{k}, \vec{t})$ as rescaled Brownian time variables along skeleton paths. The sum over $\vec{m}$ in (16.30) can be interpreted as a Riemann sum approximation to the integral defining $\hat{A}^{(r)}(k)$.

\subsection{Construction of ISE}

In this section, we present a proof that rescaled critical Poisson branching random walk converges to ISE, constructing ISE in the process. The proof 
is based on [30]. We will also derive explicit formulas for the characteristic functions of the ISE moment measures. Our proof applies directly only to the Poisson case, due to its reliance on the particular form of the generating functions derived in Theorems 15.1, 15.2 and 15.4. However, the theorem actually holds more generally, whenever the random variable $\xi$ for the offspring distribution has a finite second moment $[14,15]$.

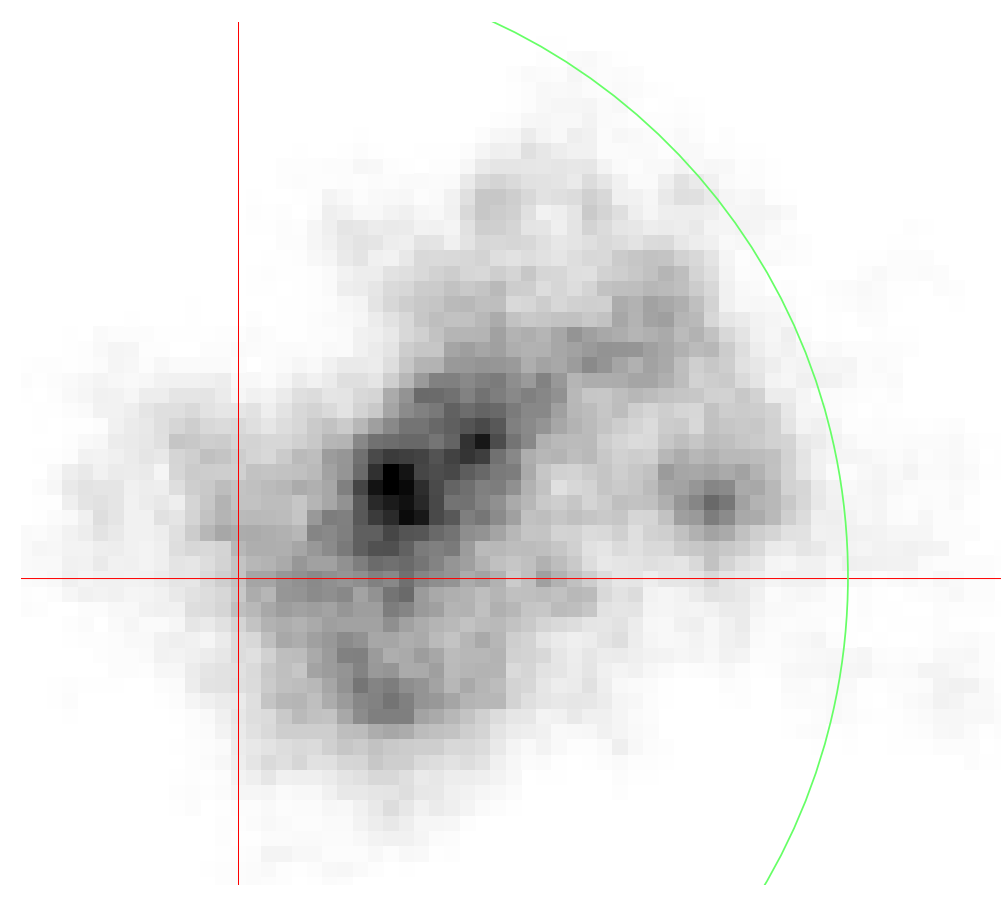

Fig. 16.1. A random mass distribution drawn from $\mu_{n}$, with $d=2$ and $n \approx 96,000$. Darker shading represents higher mass, and the arc has radius $n^{1 / 4}$.

As an important first step, we prove convergence of the moment measures of the probability measures $\mu_{n}$ on $M_{1}\left(\mathbb{R}^{d}\right)$ defined in Section 16.1.

Theorem 16.5. For $d \geq 1$ and $l \geq 1$, the moment measures $M_{n}^{(l)}$ of $\mu_{n}$ converge weakly to limiting measures $M^{(l)}$.

Proof. It suffices to prove that for $\bar{k} \in \mathbb{R}^{d l}$ the limits

$$
\lim _{n \rightarrow \infty} \hat{M}_{n}^{(l)}(\bar{k})=\hat{M}^{(l)}(\bar{k})
$$

exist for all $l \geq 1$, with $\hat{M}^{(l)}$ continuous at $\overline{0}$. By (16.5) and (15.15), the characteristic function of $M_{n}^{(l)}$ is 


$$
\begin{aligned}
\hat{M}_{n}^{(l)}(\bar{k}) & =\frac{1}{n^{l} \mathbb{P}(|T|=n)} \hat{s}_{n}^{(l+1)}\left(\bar{k} n^{-1 / 4}\right) \\
& \sim \sqrt{2 \pi} n^{-(l-3 / 2)} \hat{s}_{n}^{(l+1)}\left(\bar{k} n^{-1 / 4}\right),
\end{aligned}
$$

where $s_{n}^{(l+1)}$ is given by (16.4).

Define $b_{n}^{(r)}(\sigma ; \vec{y})$, for $r \geq 2$, by

$$
b_{n}^{(r)}(\sigma ; \vec{y})=\sum_{\vec{m}} b_{n}^{(r)}(\sigma ; \vec{y}, \vec{m})
$$

where the summand is defined in (15.23). For $r=2,3$, there is only one shape and, suppressing $\sigma$ in the notation, we have

$$
s_{n}^{(2)}(x)=b_{n}^{(2)}(x), \quad s_{n}^{(3)}\left(x_{1}, x_{2}\right)=\sum_{y \in \mathbb{Z}^{d}} b_{n}^{(3)}\left(y, x_{1}-y, x_{2}-y\right) .
$$

It then follows from (16.30) that

$$
\lim _{n \rightarrow \infty} \hat{M}_{n}^{(1)}(k)=\hat{A}^{(2)}(k)=\hat{M}^{(1)}(k)
$$

and

$$
\lim _{n \rightarrow \infty} \hat{M}_{n}^{(2)}\left(k_{1}, k_{2}\right)=\hat{A}^{(3)}\left(k_{1}+k_{2}, k_{1}, k_{2}\right)=\hat{M}^{(2)}\left(k_{1}, k_{2}\right)
$$

where $\hat{M}^{(1)}(k)$ and $\hat{M}^{(2)}\left(k_{1}, k_{2}\right)$ are defined by the second equalities. These are continuous at zero by definition.

The situation is more subtle for $l \geq 3$. To begin, we define a way of associating a $2 l-1$ component vector $\vec{k}$ to an $l$ component vector $\bar{k}$, generalizing the expression $\vec{k}=\left(k_{1}+k_{2}, k_{1}, k_{2}\right)$ in (16.37) for $l=2$. This is done as follows. Given a shape $\sigma \in \Sigma_{l+1}$, for each vertex $j$ of degree 1 in $\sigma$, other than vertex 0 , we let $\omega_{j}$ be the set of edges in $\sigma$ on the path from 0 to $j(j=1, \ldots, l)$. Given $\bar{k}=\left(k_{1} \ldots, k_{l}\right) \in \mathbb{R}^{d l}$, we define $\vec{k}(\sigma) \in \mathbb{R}^{(2 l-1) d}$ by setting its $i^{\text {th }}$ component $\vec{k}_{i}(\sigma) \in \mathbb{R}^{d}$, for $i=1, \ldots, 2 l-1$, to be

$$
\vec{k}_{i}(\sigma)=\sum_{j=1}^{l} k_{j} I\left[i \in \omega_{j}\right]
$$

where, on the right hand side, $k_{j}$ denotes the $j^{\text {th }}$ component of $\bar{k}$ and $I$ is an indicator function. For example, for $l=3$ and the shape $\sigma_{1}$ of Fig. 15.1, $\vec{k}\left(\sigma_{1}\right)=\left(k_{1}+k_{2}+k_{3}, k_{1}, k_{2}+k_{3}, k_{2}, k_{3}\right)$. We will prove $(16.32)$ with

$$
\hat{M}^{(l)}(\bar{k})=\sum_{\sigma \in \Sigma_{l+1}} \hat{A}^{(l+1)}(\vec{k}(\sigma)),
$$

which is continuous at $\overline{0}$ by definition. The limit (16.39) has been established already for $l=1,2$. 
For $l \geq 3$, we first note that (15.15) and (16.30) imply that

$$
\lim _{n \rightarrow \infty} \frac{\sum_{\sigma \in \Sigma_{l+1}} \hat{b}_{n}^{(l+1)}\left(\sigma ; \vec{k} n^{-1 / 4}\right)}{n^{l} \mathbb{P}(|T|=n)}=\sum_{\sigma \in \Sigma_{l+1}} \hat{A}^{(l+1)}(\vec{k}) .
$$

Let

$$
e_{n}^{(l+1)}(\bar{k})=\hat{s}_{n}^{(l+1)}(\bar{k})-\sum_{\sigma \in \Sigma_{l+1}} \hat{b}_{n}^{(l+1)}(\sigma ; \vec{k}(\sigma)) .
$$

In view of (16.33) and (16.40), to prove convergence of the $l^{\text {th }}$ moments, for $l \geq 3$, it suffices to show that

$$
\left|e_{n}^{(l+1)}(\bar{k})\right| \leq O\left(n^{l-2}\right) .
$$

The remainder of the proof is devoted to obtaining (16.42).

Recall the definition of $\omega_{j}=\omega_{j}(\sigma)$ above (16.38). For $\sigma \in \Sigma_{l+1}$, let

$$
Y_{\bar{x}}(\sigma)=\left\{\vec{y} \in \mathbb{R}^{d(2 l-1)}: \sum_{i \in \omega_{j}(\sigma)} y_{i}=x_{j} \forall j=1, \ldots, l\right\} .
$$

Recall the definitions above (15.23), and note that if $(T, \varphi, \bar{\imath})$ is compatible with $(\sigma ; \vec{y}, \vec{m})$, then the statements (i) $\varphi\left(i_{j}\right)=x_{j}$ for all $j=1, \ldots, l$, and (ii) $\vec{y} \in Y_{\bar{x}}(\sigma)$, are equivalent. Consequently,

$$
\begin{aligned}
e_{n}^{(l+1)}(\bar{k})= & \sum_{\bar{x}} \mathrm{e}^{\mathrm{i} \bar{k} \cdot \bar{x}}\left[s_{n}^{(l+1)}(\bar{x})-\sum_{\sigma \in \Sigma_{l+1}} \sum_{\vec{y} \in Y_{\bar{x}}(\sigma)} \sum_{\vec{m}} b_{n}^{(l+1)}(\sigma ; \vec{y}, \vec{m})\right] \\
= & \sum_{\bar{x}} \mathrm{e}^{\mathrm{i} \bar{k} \cdot \bar{x}} \sum_{(T, \varphi):|T|=n} \mathbb{P}(T, \varphi) \sum_{i_{1}, \ldots, i_{l} \in T}\left[\prod_{j=1}^{l} I\left[\varphi\left(i_{j}\right)=x_{j}\right]\right] \\
& \times\left[1-\sum_{\sigma \in \Sigma_{l+1}} \sum_{\vec{y} \in Y_{\bar{x}}(\sigma)} \sum_{\vec{m}} I[(T, \varphi, \bar{\imath}) \text { comp. with }(\sigma ; \vec{y}, \vec{m})] .\right.
\end{aligned}
$$

The last line of the above expression need not be zero. For example, let $T$ be the unique tree having just two vertices, let $\varphi$ be the embedding of $T$ which maps the root of $T$ to 0 and the other vertex of $T$ to a neighbour $e_{1}$ of the origin, let $i_{1}$ and $i_{2}$ be the root of $T$, and let $i_{3}$ be the other vertex of $T$. In this example, the subtracted sum in (16.44) is equal to 3 , due to the terms with $\sigma=\sigma_{1}, y_{1}=\left(0,0,0,0, e_{1}\right) ; \sigma=\sigma_{2}, y_{2}=\left(0,0,0,0, e_{1}\right)$; and $\sigma=\sigma_{3}$, $y_{3}=\left(0, e_{1}, 0,0,0\right)$, where the shapes $\sigma_{i}$ are given in Fig. 15.1.

However, given $(T, \varphi, \bar{\imath})$, and given $\sigma \in \Sigma_{l+1}$, if $(T, \varphi, \bar{\imath})$ is compatible with $(\sigma ; \vec{y}, \vec{m})$ then $\vec{y}$ and $\vec{m}$ are uniquely determined by $(T, \varphi, \bar{\imath})$ and $\sigma$. Thus for each $\sigma$ contributing to the last line of (16.44), there is exactly one $\vec{y}$ and 
one $\vec{m}$ that contribute to the sums over $\vec{y}$ and $\vec{m}$. Also, each $(T, \varphi, \bar{\imath})$, such that the reduced skeleton $\beta_{B}(T, \bar{\imath})$ of $(T, \bar{\imath})$ is isomorphic to a shape in $\Sigma_{l+1}$ (rather than to a strict subshape), receives a contribution to (16.44) due to that shape and only to that shape. Therefore, writing $\Lambda_{\sigma}$ for the set of all strict subshapes of $\sigma \in \Sigma_{l+1}$,

$$
\begin{aligned}
\left|e_{n}^{(l+1)}(\bar{k})\right| \leq & \sum_{\sigma \in \Sigma_{l+1}} \sum_{\lambda \in \Lambda_{\sigma}} \sum_{\bar{x}} \sum_{(T, \varphi):|T|=n} \mathbb{P}(T, \varphi) \\
& \times \sum_{i_{1}, \ldots, i_{l} \in T}\left[\prod_{j=1}^{l} I\left[\varphi\left(i_{j}\right)=x_{j}\right]\right] I\left[\beta_{B}(T, \bar{\imath}) \simeq \lambda\right] \\
= & \sum_{\sigma \in \Sigma_{l+1}} \sum_{\lambda \in \Lambda_{\sigma}} \sum_{(T, \varphi):|T|=n} \mathbb{P}(T, \varphi) \sum_{i_{1}, \ldots, i_{l} \in T} I\left[\beta_{B}(T, \bar{\imath}) \simeq \lambda\right] .
\end{aligned}
$$

Let $f_{n}^{(l+1)}$ denote the right hand side of (16.45). It suffices to show that $f_{n}^{(l+1)} \leq O\left(n^{l-2}\right)$. For this, we introduce the generating function $F^{(l+1)}(z)=$ $\sum_{n=1}^{\infty} f_{n}^{(l+1)} z^{n}$. This is a sum of terms of the form $\hat{b}(\lambda)$ (defined below (15.29)), where $\lambda$ is a strict subshape and all $k_{j}=0, \zeta_{j}=1$. By (15.31), (15.27) and $(16.8)$,

$$
|\hat{b}(\lambda)| \leq O(1) \prod_{j \in e(\lambda)} \frac{1}{|1-z|^{1 / 2}}
$$

and hence $\left|F^{(l+1)}(z)\right| \leq O\left(|1-z|^{-(l-1)}\right)$ uniformly in $|z|<1$, where the power $l-1=\frac{1}{2}(2 l-2)$ arises because at least one of the $2 l-1$ skeleton paths is contracted. Then Exercise 7.7 implies the desired bound $f_{n}^{(l+1)} \leq O\left(n^{l-2}\right)$.

Using Theorem 16.5, we can now prove convergence of $\mu_{n}$ to a limiting measure $\mu_{\text {ISE }}$ on $M_{1}\left(\mathbb{R}^{d}\right)$, thereby constructing ISE. We will use the fact that $M_{1}\left(\mathbb{R}^{d}\right)$ is a metric space (in fact it is a Polish space) whose metric induces the weak topology; references for this are given in [177, p. 148].

Theorem 16.6. For $d \geq 1$, there is a measure $\mu_{\mathrm{ISE}}$ on $M_{1}\left(\mathbb{R}^{d}\right)$ such that $\mu_{n}$ converges weakly to $\mu_{\mathrm{ISE}}$ on $M_{1}\left(\mathbb{R}^{d}\right)$.

Proof. We will prove that $\mu_{n}$ is relatively compact, i.e., that every subsequence of $\mu_{n}$ contains a weakly convergent subsequence (see [24]). Given a subsequence of $\mu_{n}$, relative compactness implies that some further subsequence converges to a limit. In principle, this limit could depend on the subsequence. But Theorem 16.5 implies that the limit has moment measures $M^{(l)}$, which determines it uniquely. ${ }^{16}$ We call the common limit $\mu_{\text {ISE }}$.

${ }^{16}$ It is a consequence of $[60$, Lemma 2.4 .1$]$ that moment measures uniquely determine a measure on $M_{1}\left(\dot{\mathbb{R}}^{d}\right)$, where $\dot{\mathbb{R}}^{d}$ is the one-point compactification of $\mathbb{R}^{d}$. Since the moments have no mass at infinity, they therefore uniquely determine a measure on $M_{1}\left(\mathbb{R}^{d}\right)$. 
To prove relative compactness, we apply Theorem II.4.1 of [177]. This theorem actually applies in the more general context of convergence of measurevalued processes. We have no time-dependence here, so our processes are constant. Condition (ii) of Theorem II.4.1 is vacuous for our purpose. ${ }^{17}$ It suffices to verify Condition (i) of Theorem II.4.1, which asserts that given $\epsilon>0$ there is a compact set $K_{\epsilon}$ such that

$$
\sup _{n} \mu_{n}\left(\nu\left(K_{\epsilon}^{c}\right)>\epsilon\right)<\epsilon
$$

Take $K_{\epsilon}=B_{R}=\left\{x \in \mathbb{R}^{d}:|x| \leq R\right\}$, where we will choose $R=R(\epsilon)$ below. Let $\psi_{R}$ be a smooth function which takes values in $[0,1]$, which is 1 on $B_{R}^{c}$, and which is 0 on $B_{R-1}$. Then

$$
\begin{aligned}
\mu_{n}\left(\nu\left(K_{\epsilon}^{c}\right)>\epsilon\right) & \leq \epsilon^{-1} \mathbb{E}_{\mu_{n}}\left[\nu\left(K_{\epsilon}^{c}\right)\right] \\
& \leq \epsilon^{-1} \mathbb{E}_{\mu_{n}}\left[\nu\left(\psi_{R}\right)\right] \\
& =\epsilon^{-1} \int_{\mathbb{R}^{d}} \psi_{R}(x) \mathrm{d} M_{n}^{(1)}(x) .
\end{aligned}
$$

By Theorem 16.5,

$$
\begin{aligned}
\lim _{n \rightarrow \infty} \int_{\mathbb{R}^{d}} \psi_{R}(x) \mathrm{d} M_{n}^{(1)}(x) & =\int_{\mathbb{R}^{d}} \psi_{R}(x) \mathrm{d} M^{(1)}(x) \\
& \leq \int_{|x|>R-1} \mathrm{~d} M^{(1)}(x) .
\end{aligned}
$$

Since $\int_{\mathbb{R}^{d}} \mathrm{~d} M^{(1)}(x)=1$, the right hand side is less than $\epsilon^{2} / 2$ if we choose $R$ large depending on $\epsilon$, and therefore the integral on the left hand side is less than $\epsilon^{2}$ for this $R$ if we choose $n$ large depending on $R$ and hence on $\epsilon$, say $n \geq n_{0}(\epsilon)$. We have proved that

$$
\sup _{n \geq n_{0}(\epsilon)} \mu_{n}\left(\nu\left(K_{\epsilon}^{c}\right)>\epsilon\right)<\epsilon
$$

and we are left with $n<n_{0}(\epsilon)$.

Note that (16.50) continues to hold if we increase $R$, since this only decreases the left hand side. However, if we now increase $R$ (if necessary) until it exceeds $n_{0}(\epsilon)$, then $\nu\left(K_{\epsilon}^{c}\right)=0$ almost surely under $\mu_{n}$ for any $n<n_{0}(\epsilon)$, since under $\mu_{n}$ it is the case that $\nu$ is supported on the ball in $\mathbb{R}^{d}$ of radius $n^{1-1 / 4}=n^{3 / 4}$. Thus (16.50) implies (16.47).

\subsection{Lattice trees and ISE}

It was conjectured by Aldous [15] that ISE describes the scaling limit of lattice trees in dimensions $d>8$. In this section, we discuss the theorem of [62]

\footnotetext{
${ }^{17}$ We take $\mathrm{e}^{\mathrm{i} k \cdot x}$ as our separating class and observe that its integral with respect to a probability measure $\nu$ is bounded by 1 , so $\nu\left(\mathrm{e}^{\mathrm{i} k \cdot x}\right)$ is relatively compact.
} 
that this is the case for sufficiently spread-out lattice trees, following the presentation of [188].

Basic definitions for lattice trees were given in Section 7.1. In particular, $t_{n}^{(1)}$ was defined to be the number of $n$-bond lattice trees that contain the origin, and $t_{n}^{(2)}(x)$ was defined to be the number of $n$-bond lattice trees that contain the origin and $x$. Now we also define functions $t_{n}^{(r)}(\sigma ; \vec{y}, \vec{s})$ for $r \geq 2$, using the terminology introduced above Theorem 15.4 for branching random walk, which we recast here in a form appropriate for lattice trees.

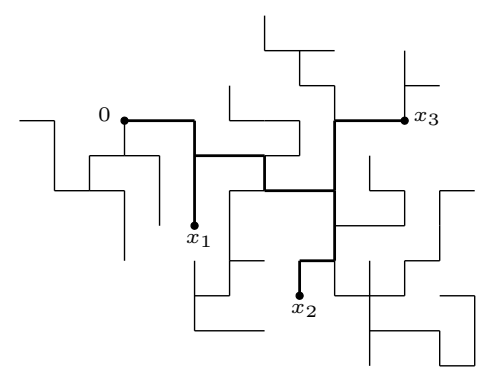

Fig. 16.2. A 2-dimensional lattice tree contributing to $t_{78}^{(4)}\left(\sigma_{1} ; \vec{y}, \vec{m}\right)$, with $\sigma_{1}$ depicted in Fig. 15.1, $\vec{y}=((2,-1),(0,-2),(4,-1),(-1,-3),(2,2)), \vec{m}=(3,2,5,4,4)$.

Given a lattice tree $T$ containing the vertices $0, x_{1}, \ldots, x_{r-1}$ (not necessarily distinct), we define the skeleton $B$ to be the subtree of $T$ spanning $0, x_{1}, \ldots, x_{r-1}$. Let $\beta_{B}$ be the tree (not a lattice tree) obtained by ignoring vertices in $B$ of degree 2 , other than $0, x_{1}, \ldots, x_{r-1}$. Let $\sigma \in \Sigma_{r}$, $\vec{m}=\left(m_{1}, \ldots, m_{2 r-3}\right)$ for non-negative integers $m_{j}$, and $\vec{y}=\left(y_{1}, \ldots, y_{2 r-3}\right)$ for $y_{j} \in \mathbb{Z}^{d}$. We say that $(T ; \bar{x})$ is compatible with $(\sigma ; \vec{y}, \vec{m})$ if the following hold:

1. $\beta_{B}$ is isomorphic to a subshape $\lambda$ of $\sigma$ (in which case the paths of the skeleton $B$ have an induced labelling).

2. Let $l_{j}>0$ denote the length of the skeleton path labelled $j$, and let $l_{j}=0$ for any edge in $\sigma$ that is not in $\lambda$. Then $l_{j}=m_{j}$ for each $j=1, \ldots, 2 r-3$.

3 . The skeleton path (oriented away from the origin) labelled $j$ undergoes the displacement $y_{j}$ for each $j$ labelling an edge in $\lambda$, and $y_{j}=0$ for any edge $j$ in $\sigma$ that is not in $\lambda$.

Then we define $t_{n}^{(r)}(\sigma ; \vec{y}, \vec{m})$ to be the number of $n$-bond lattice trees $T$, containing the origin, for which there are vertices $x_{1}, \ldots, x_{r-1} \in T$ such that $(T ; \bar{x})$ is compatible with $(\sigma ; \vec{y}, \vec{m})$. (Note that $\bar{x}$ is in fact uniquely determined by $\sigma$ and $\vec{y}$.) See Fig. 16.2. We also define

$$
t_{n}^{(r)}(\sigma ; \vec{y})=\sum_{\vec{m}} t_{n}^{(r)}(\sigma ; \vec{y}, \vec{m})
$$


where the sum over $\vec{m}$ denotes a sum over the non-negative integers $m_{j}$. For $r=2,3$ there is a unique shape and we sometimes omit it from the notation. We also define the generating functions

$$
G_{z, \vec{\zeta}}^{(r)}(\sigma ; \vec{y})=\sum_{n=0}^{\infty} \sum_{\vec{m}} t_{n}^{(r)}(\sigma ; \vec{y}, \vec{m}) z^{n} \prod_{j=1}^{2 r-3} \zeta_{j}^{m_{j}}
$$

for $r \geq 2$. For $r \geq 3$, these generating functions keep track of more information than those defined in (7.20). The sum over $\vec{y} \in \mathbb{R}^{d(2 r-3)}$ of (16.52) is finite for $|z|<z_{c}$ and $\left|\zeta_{j}\right| \leq 1$, for all $r$.

The critical exponents $\eta$ and $\gamma$ were defined in (7.8) and (7.6) by

$$
\hat{G}_{z_{c}, 1}^{(2)}(k) \sim \operatorname{const} \frac{1}{|k|^{2-\eta}} \text { as } k \rightarrow 0, \quad \hat{G}_{z, 1}^{(2)}(0) \sim \operatorname{const} \frac{1}{\left(1-z / z_{c}\right)^{\gamma}} \text { as } z \rightarrow z_{c} .
$$

Their mean-field values are $\eta=0$ and $\gamma=\frac{1}{2}$. By analogy with (16.9), we might hope and expect that in dimensions $d>8$,

$$
\hat{G}_{z, \zeta}^{(2)}(k)=\frac{C_{0}}{D_{0}^{2}|k|^{2}+2^{3 / 2}\left(1-z / z_{c}\right)^{1 / 2}+2 T_{0}(1-\zeta)}+\text { error }
$$

where $C_{0}, D_{0}, T_{0}$ are positive constants, and where the error term is of lower order than the main term in some suitable sense, as $k \rightarrow 0, z \rightarrow z_{c}$, and $\zeta \rightarrow 1$. Furthermore, by analogy with Theorem 15.4, we might expect that for $d>8$ there is approximate independence of the form

$$
\hat{G}_{z, \vec{\zeta}}^{(r)}(\sigma ; \vec{k})=V^{r-2} \prod_{j=1}^{2 r-3} \hat{G}_{z, \zeta_{j}}^{(2)}\left(k_{j}\right)+\text { error }
$$

where $V$ is a finite positive constant which encompasses the self-avoidance interactions of lattice trees in a renormalized vertex factor (cf. (7.41), and also Section 5.3 and Theorems 6.9 and 6.10).

For the nearest-neighbour model with $d$ sufficiently large, and for spreadout models for $d>8$ with $L$ sufficiently large, relations of the form (16.54) and (16.55) are proved in [62], for all $r \geq 2$ if $\vec{\zeta}=\overrightarrow{1}$ and for $r=2,3$ for general $\vec{\zeta}$. The results given below arise as a consequence. For the statement of the results, we define

$$
p_{n}^{(r)}(\sigma ; \vec{y})=\frac{t_{n}^{(r)}(\sigma ; \vec{y})}{\sum_{\sigma \in \Sigma_{m}} \hat{t}_{n}^{(r)}(\sigma ; \overrightarrow{0})},
$$

which is a probability measure on $\Sigma_{r} \times \mathbb{Z}^{d(2 r-3)}$. The following theorem from $[61,62]$, whose proof extends the methods of $[95,99]$, shows that (16.56) has the corresponding ISE density as its scaling limit in high dimensions. In its statement, the scaling of $\vec{k}$ corresponds to scaling down the lattice spacing by a multiple of $n^{-1 / 4}$. 
Theorem 16.7. Let $r \geq 2, \sigma \in \Sigma_{r}, k_{j} \in \mathbb{R}^{d}(j=1, \ldots, 2 r-3)$, and let $D_{0}$ be given by (7.18). For nearest-neighbour lattice trees in sufficiently high dimensions $d \geq d_{0}$, and for spread-out lattice trees with $d>8$ and $L$ sufficiently large depending on $d$, there is a constant ${ }^{18} c_{1}$ depending on $d$ and $L$ such that as $n \rightarrow \infty$

$$
\hat{t}_{n}^{(r)}\left(\sigma ; \vec{k} D_{0}^{-1} n^{-1 / 4}\right) \sim c_{1} n^{r-5 / 2} z_{c}^{-n} \hat{A}^{(r)}(\vec{k}) .
$$

In particular,

$$
\lim _{n \rightarrow \infty} \hat{p}_{n}^{(r)}\left(\sigma ; \vec{k} D_{0}^{-1} n^{-1 / 4}\right)=\hat{A}^{(r)}(\vec{k})
$$

It is a corollary of Theorem 16.7 that high-dimensional lattice trees converge weakly to ISE, as we now explain. Given an $n$-bond lattice tree $T$ containing the origin, we define $\nu_{T}$ to be the probability measure on $\mathbb{R}^{d}$ which for each of the $n+1$ vertices $x \in T$ assigns mass $(n+1)^{-1}$ to $x D_{0}^{-1} n^{-1 / 4}$ in $\mathbb{R}^{d}$. We then define a probability measure $\mu_{n}^{\mathrm{LT}}$ on $M_{1}\left(\mathbb{R}^{d}\right)$, supported on the $\nu_{T}$, by $\mu_{n}^{\mathrm{LT}}\left(\left\{\nu_{T}\right\}\right)=\left(t_{n}^{(1)}\right)^{-1}$ for each $n$-bond lattice tree $T$ containing 0 . In this way, $n$-bond lattice trees induce a random probability measure on $\mathbb{R}^{d}$.

Corollary 16.8. For nearest-neighbour lattice trees in sufficiently high dimensions $d \geq d_{0}$, and for spread-out lattice trees with $d>8$ and $L$ sufficiently large, $\mu_{n}^{\mathrm{LT}}$ converges weakly to $\mu_{\mathrm{ISE}}$, as measures on $M_{1}\left(\mathbb{R}^{d}\right)$.

The argument leading from Theorem 16.7 to Corollary 16.8 is a straightforward adaptation of the proof of Theorem 16.6. The details are made explicit in [188, Appendix A], where weak convergence of $\mu_{n}^{\mathrm{LT}}$ to $\mu_{\mathrm{ISE}}$ is proved. ${ }^{19}$

For a more refined statement than Theorem 16.7, we define

$$
p_{n}^{(r)}(\sigma ; \vec{y}, \vec{m})=\frac{t_{n}^{(r)}(\sigma ; \vec{y}, \vec{m})}{\sum_{\sigma \in \Sigma_{r}} \hat{t}_{n}^{(r)}(\sigma ; \overrightarrow{0})}
$$

which is a probability measure on $\Sigma_{r} \times \mathbb{Z}^{d(2 r-3)} \times \mathbb{Z}_{+}^{2 r-3}$. The following theorem is due to $[61,62]$. In its statement we drop $\sigma$, since there is a unique shape for $r=2,3$.

Theorem 16.9. Let $r=2$ or $r=3, k_{j} \in \mathbb{R}^{d}$, and $t_{j} \in(0, \infty)(j=1, \ldots, 2 r-$ 3). For nearest-neighbour lattice trees in sufficiently high dimensions $d \geq d_{0}$, and for spread-out lattice trees with $d>8$ and $L$ sufficiently large depending on $d$, there is a constant $T_{0}$ depending on $d$ and $L$, such that as $n \rightarrow \infty$,

$$
\hat{t}_{n}^{(r)}\left(\vec{k} D_{0}^{-1} n^{-1 / 4},\left\lfloor\vec{t} T_{0} n^{1 / 2}\right\rfloor\right) \sim c_{1} T_{0}^{-(2 r-3)} n^{-1} z_{c}^{-n} \hat{a}^{(r)}(\vec{k}, \vec{t}),
$$

${ }_{18}$ The constant $c_{1}$ is equal to the constant $A$ of Theorems 7.3 and 7.4 , but $A$ is already in use with a different meaning in (16.57).

${ }^{19}$ In [188], convergence is proved as measures on probability measures on the onepoint compactification of $\mathbb{R}^{d}$, but the one-point compactification can be avoided as in the proof of Theorem 16.6. 
where $c_{1}$ and $D_{0}$ are the constants of Theorem 16.7. In particular,

$$
\lim _{n \rightarrow \infty}\left(T_{0} n^{1 / 2}\right)^{2 r-3} \hat{p}_{n}^{(r)}\left(\vec{k} D_{0}^{-1} n^{-1 / 4},\left\lfloor\vec{t} T_{0} n^{1 / 2}\right\rfloor\right)=\hat{a}^{(r)}(\vec{k}, \vec{t}) .
$$

It would be very surprising if Theorem 16.9 did not extend to all $r \geq 2$, but technical difficulties arise for $r \geq 4$ and the theorem has been proved ${ }^{20}$ only for $r=2$ and $r=3$. Theorem 16.9 indicates that, at least for $r=2$ and $r=3$, skeleton paths with length of order $n^{1 / 2}$ are typical. This is Brownian scaling, since distance is scaled as $n^{1 / 4}$, and the variables $t_{j}$ correspond to rescaled skeleton time variables.

See Section 17.5 for further results in this direction. See also [182] for a study of the time evolution of ISE.

\subsection{Critical Percolation and ISE}

In this section, we discuss results of $[103,104,105]$ which relate critical percolation and ISE above the upper critical dimension, following the presentation of [188].

Consider independent Bernoulli bond percolation on $\mathbb{Z}^{d}$, nearest-neighbour or spread-out, with $p$ fixed and equal to its critical value $p_{c}$. The basic definitions are laid out in Section 9. As usual, we let $C(0)$ denote the random set of vertices connected to 0 , of cardinality $|C(0)|$. Let

$$
\tau^{(2)}(x ; n)=\mathbb{P}_{p_{c}}(C(0) \ni x,|C(0)|=n)
$$

denote the probability at the critical point that the origin is connected to $x$ via a cluster containing $n$ vertices, and define the generating function

$$
\tau_{z}^{(2)}(x)=\sum_{n=1}^{\infty} \tau^{(2)}(x ; n) z^{n}
$$

which converges absolutely if $|z| \leq 1$. Assuming no infinite cluster at $p_{c}$, $\tau_{1}^{(2)}(x)$ is the probability that 0 is connected to $x$. Also, if we set $z=1-\gamma$, then $\hat{\tau}_{z}^{(2)}(0)$ is equal to $\chi(p, \gamma)$ of $(9.29)$.

The definitions of the critical exponents $\eta$ and $\delta$ in (9.17) and (9.18), together with (9.29), suggest that

$$
\hat{\tau}_{1}^{(2)}(k) \sim \frac{c_{1}}{|k|^{2-\eta}} \text { as } k \rightarrow 0, \quad \hat{\tau}_{z}^{(2)}(0) \sim \frac{c_{2}}{(1-z)^{1-1 / \delta}} \text { as } z \rightarrow 1^{-} .
$$

Inserting the mean-field values $\eta=0$ and $\delta=2$, the simplest combination of the above asymptotic relations for $d>6$ would be

$$
\hat{\tau}_{z}^{(2)}(k)=\frac{C_{2}}{D_{2}^{2}|k|^{2}+2^{3 / 2}(1-z)^{1 / 2}}+\text { error, }
$$

\footnotetext{
${ }^{20}$ The statement of Theorem 16.9 for $r=3$ in $[11,12]$ incorrectly included the case where $t_{j}=0$ for some $j$.
} 
for some constants $C_{2}, D_{2}$. This is analogous to (16.8) with $\zeta=1$. Theorem 11.3 shows that (16.65) is correct for sufficiently spread-out percolation above six dimensions, in the sense that there are functions $\epsilon_{1}(z)$ and $\epsilon_{2}(k)$ with $\lim _{z \rightarrow 1^{-}} \epsilon_{1}(z)=\lim _{k \rightarrow 0} \epsilon_{2}(k)=0$, and constants $C_{2}$ and $D_{2}$ depending on $d$ and $L$, such that

$$
\hat{\tau}_{z}^{(2)}(k)=\frac{C_{2}}{D_{2}^{2}|k|^{2}+2^{3 / 2}(1-z)^{1 / 2}}[1+\epsilon(z, k)]
$$

for real $z \in[0,1)$, with $|\epsilon(z, k)| \leq \epsilon_{1}(z)+\epsilon_{2}(k)$.

We have seen in (16.19) that the coefficient of $z^{n}$ in $\left[|k|^{2}+2^{3 / 2}(1-z)^{1 / 2}\right]^{-1}$ is intimately related to $\hat{A}^{(2)}(k)$ and hence to ISE. Thus (16.66) is highly suggestive that ISE occurs as a scaling limit for percolation, but the control of the error term in (16.66) is too weak to obtain bounds on $\hat{\tau}^{(2)}\left(k D_{2}^{-1} n^{-1 / 4} ; n\right)$ via contour integration. However, for the nearest-neighbour model in sufficiently high dimensions, better control of the error terms has been obtained, for complex $z$ with $|z|<1$, leading to the following theorem [103, 105]. The theorem also gives an asymptotic formula for the three-point function

$$
\tau^{(3)}(x, y ; n)=\mathbb{P}_{p_{c}}(x, y \in C(0),|C(0)|=n),
$$

in terms of its Fourier transform

$$
\hat{\tau}^{(3)}(k, l ; n)=\sum_{x, y \in \mathbb{Z}^{d}} \tau^{(3)}(x, y ; n) \mathrm{e}^{\mathrm{i} k \cdot x+\mathrm{i} l \cdot y} .
$$

Theorem 16.10. Let $p=p_{c}$. Fix $k, l \in \mathbb{R}^{d}$ and any $\epsilon \in\left(0, \frac{1}{2}\right)$. There is a $d_{0}$ such that for nearest-neighbour percolation with $d \geq d_{0}$, there are constants $C_{2}, D_{2}$ (depending on d) such that as $n \rightarrow \infty$

$$
\begin{aligned}
\hat{\tau}^{(2)}\left(k D_{2}^{-1} n^{-1 / 4} ; n\right) & =\frac{C_{2}}{\sqrt{8 \pi n}} \hat{A}^{(2)}(k)\left[1+O\left(n^{-\epsilon}\right)\right], \\
\hat{\tau}^{(3)}\left(k D_{2}^{-1} n^{-1 / 4}, l D_{2}^{-1} n^{-1 / 4} ; n\right) & =\frac{C_{2}}{\sqrt{8 \pi}} n^{1 / 2} \hat{A}^{(3)}(k+l, k, l)\left[1+O\left(n^{-\epsilon}\right)\right] .
\end{aligned}
$$

It follows from (16.69) that

$$
\begin{aligned}
\mathbb{P}_{p_{c}}(|C(0)|=n) & =n^{-1} \hat{\tau}^{(2)}(0 ; n) \\
& =C_{2}(8 \pi)^{-1 / 2} n^{-3 / 2}\left[1+O\left(n^{-\epsilon}\right)\right] .
\end{aligned}
$$

Comparing with (9.19), this is a statement that the critical exponent $\delta$ is equal to 2 in high dimensions.

The proof of (16.70) considers the generating function

$$
\hat{\tau}_{z}^{(3)}(k, l)=\sum_{n=1}^{\infty} \tau^{(3)}(k, l ; n) z^{n},
$$


and shows that there is a positive constant $V$ such that

$$
\hat{\tau}_{z}^{(3)}(k, l)=V \hat{\tau}_{z}^{(2)}(k+l) \hat{\tau}_{z}^{(2)}(k) \hat{\tau}_{z}^{(2)}(l)+\text { error, }
$$

where the error term is treated using Exercise 7.7. The variables in (16.70) are arranged schematically as:

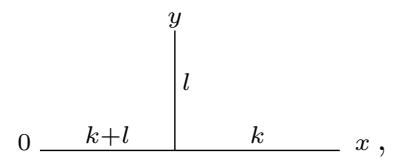

and (16.73) asserts that the three-point function approximately decouples into the vertex factor $V$ times a product of three two-point functions. An asymptotic relation in the spirit of (16.73), with $k=l=0$, was conjectured for $d>6$ already in [12].

We expect that Theorem 16.10 should extend to general $r$-point functions, for all $r \geq 2$, but this has not been proven. This is essentially the conjecture of [103] that the scaling limit of the incipient infinite cluster is ISE for $d>6$. We now discuss this conjecture in more detail.

Fix $n$ and let $S$ be a site lattice animal containing $n$ sites, i.e., a set of $n$ vertices in $\mathbb{Z}^{d}$ any two of which are connected by a sequence of vertices in $S$ separated by unit distance. Supposing that $S$ contains the origin, define the probability measure $\nu_{S} \in M_{1}\left(\mathbb{R}^{d}\right)$ to assign mass $n^{-1}$ to $x D_{2}^{-1} n^{-1 / 4}$, for each $x \in S$. We define $\mu_{n}^{\text {Perc }}$ to be the probability measure on $M_{1}\left(\mathbb{R}^{d}\right)$ which assigns probability $\mathbb{P}_{p_{c}}(C(0)=S|| C(0) \mid=n)$ to $\nu_{S}$, for each such $S$. We regard the limit of $\mu_{n}^{\text {Perc }}$, as $n \rightarrow \infty$, as the scaling limit of the incipient infinite cluster. This is related to Kesten's definitions of the incipient infinite cluster [142] (see Section 11.3), but here we are conditioning the cluster to have a fixed large size rather than to reach a distant hyperplane, and, more importantly, here we are taking the lattice spacing to zero as $n \rightarrow \infty$. The conjecture of [103] is that, as in Corollary 16.8 above, $\mu_{n}^{\text {Perc }}$ converges weakly to $\mu_{\text {ISE }}$ for $d>6$.

The conjecture is supported by Theorem 16.10. In fact, the characteristic functions $\hat{M}_{n}^{(1)}(k)$ and $\hat{M}_{n}^{(2)}(k, l)$ of the first and second moment measures $M_{n}^{(1)}$ and $M_{n}^{(2)}$ of $\mu_{n}^{\text {Perc }}$ are given by

$$
\begin{aligned}
\hat{M}_{n}^{(1)}(k) & =\frac{\hat{\tau}^{(2)}\left(k D_{2}^{-1} n^{-1 / 4} ; n\right)}{\hat{\tau}^{(2)}(0 ; n)}, \\
\hat{M}_{n}^{(2)}(k, l) & =\frac{\hat{\tau}^{(3)}\left(k D_{2}^{-1} n^{-1 / 4}, l D_{2}^{-1} n^{-1 / 4} ; n\right)}{\hat{\tau}^{(3)}(0,0 ; n)},
\end{aligned}
$$

and in high dimensions these converge respectively to the characteristic functions $\hat{A}^{(2)}(k)$ and $\hat{A}^{(3)}(k+l, k, l)$ of the corresponding ISE moments, by Theorem 16.10 (see also (16.39)).

From this point of view, the IIC is regarded as a cluster in $\mathbb{R}^{d}$ arising in the scaling limit, represented by ISE. ISE is almost surely supported on 
a compact subset of $\mathbb{R}^{d}$, but on the scale of the lattice, this corresponds to an infinite cluster. The scaling here is consistent with the fact, discussed in Section 11.4.4, that in high dimensions the largest cluster in a box of radius $r$ with bulk boundary condition has size of order $r^{4}$, and suggests that the largest cluster in a large box looks like an ISE cluster. In Section 17.3, a related but different construction is discussed for oriented percolation, where the IIC is first constructed as an infinite object on the lattice, and then the scaling limit of this is taken. This order of limits naturally produces an infinite continuous object, rather than a compact one.

ISE was defined in Section 16.3 as the law of a random probability measure on $\mathbb{R}^{d}$. However, ISE can also be formulated in such a way as to contain more detailed information including the structure of paths joining any finite set of points in the cluster, with these described by the functions $a^{(r)}(\vec{k}, \vec{t})$. See [152]. This is consistent with the approach of $[4,5,6,8]$ to the scaling limit, although here our focus is on a single percolation cluster, rather than on many clusters as in those references.

\section{Super-Brownian Motion}

Super-Brownian motion (SBM) is an active field in probability theory and has an extensive literature. Introductory accounts can be found in $[152,177]$, and a nontechnical introduction is [189]. SBM is a continuous time Markov process whose state space is the set of finite measures on $\mathbb{R}^{d}$. The subject can be technical, and some of the techniques used to study SBM require tools that many researchers interested in statistical mechanics do not have handy in their kit. Our account is introductory, and develops only those aspects that are essential for our specific purposes. In particular, attention is limited to the canonical measure of SBM.

In recent years, it has been realized that SBM is the scaling limit of several interacting particle systems at criticality. One aspect of this is the fact, already discussed in Sections 16.4-16.5, that ISE describes the scaling limit of lattice trees in dimensions $d>8$ and of critical percolation clusters in dimensions $d>6$. In this section, we discuss results showing that the canonical measure of SBM describes the scaling limit of critical oriented percolation for $d+1>4+1$, of the critical contact process for $d>4$ (and also in lower dimensions when the infection range is suitably unbounded), and of lattice trees for $d>8$. In addition, SBM is the scaling limit of the voter model in dimensions $d \geq 2$ $[37,58,59]$, but the voter model will not be discussed further here. The relation between super-Brownian motion and the voter model, contact process, and oriented percolation is discussed in the introductory article [178].

\subsection{The Canonical Measure of SBM}

The canonical measure of SBM describes the scaling limit of critical branching random walk, started from a single particle at the origin as in the construction 
of ISE. With ISE, we conditioned critical branching random walk clusters to have exactly $n$ particles, assigned mass $n^{-1}$ to each particle, rescaled space by $n^{-1 / 4}$, and let $n \rightarrow \infty$. The result was a random probability measure on $\mathbb{R}^{d}$. For the canonical measure of SBM we will scale differently.

Let $(T, \varphi)$ be a critical branching random walk configuration with law (15.8), as in Section 15.2. We make no assumption about the total number of vertices in $T$. Typically, $T$ will have few vertices, but we are most interested in the rare trees that are long-lived. Let $T_{m}$ denote the set of vertices at graph distance $m$ from the root of $T$. We refer to $T_{m}$ as the $m^{\text {th }}$ generation of $T$. Since $T$ is almost surely finite, eventually $T_{m}=\varnothing$.

We introduce a scaling variable $n$, which is a large integer that will be sent to infinity. Four simultaneous scalings are made, as follows.

- Scaling of time. We define a time variable $t=\frac{m}{n}$. This fixes attention on the individuals $T_{m}$ in generations $m$ of order $n$.

- Scaling of space. In a configuration $(T, \varphi)$, an individual in generation $n$ (if $T$ lives so long) is mapped by $\varphi$ to the endpoint of an $n$-step random walk path. Such a point is typically at distance order $n^{1 / 2}$ from the origin, so we rescale the lattice to $n^{-1 / 2} \mathbb{Z}^{d}$.

- Scaling of mass. A critical tree that survives to a generation of order $n$ typically has order $n$ members in that generation. To obtain a generation mass of order 1, we assign mass $n^{-1}$ to each embedded vertex.

- Scaling of probability. By a theorem of Kolmogorov, the probability that a critical tree survives for order $n$ generations is of order $n^{-1}$. The long-lived trees of interest thus have a vanishingly small probability. To compensate, we multiply probabilities by $n$, so that survival to order $n$ generations will have measure of order 1 , rather than probability of order $n^{-1}$. This produces an unnormalized measure, rather than a probability measure.

The above is carried out, in detail, as follows. Given a critical branching random walk configuration $(T, \varphi)$, we rescale the lattice to $n^{-1 / 2} \mathbb{Z}^{d}$, and denote by $R_{n}^{(m / n)}$ the mass distribution in $n^{-1 / 2} \mathbb{Z}^{d}$ of the $m^{\text {th }}$ generation $T_{m}$ of $T$. Explicitly, $R_{n}^{(m / n)}$ is the discrete finite measure on $\mathbb{R}^{d}$ that places mass $n^{-1}$ at each embedded vertex of $T_{m}$, with multiplicity, i.e.,

$$
R_{n}^{(m / n)}=\frac{1}{n} \sum_{i \in T:|i|=m} \delta_{n^{-1 / 2} \varphi(i)} .
$$

Note that $R_{n}^{(m / n)}$ need not be a probability measure. The measure $R_{n}^{(m / n)}$ is a random measure. Let $\mathcal{R}_{n}^{(m / n)}$ denote the probability law of this random measure. Thus $\mathcal{R}_{n}^{(m / n)}$ is a probability measure on the space $M_{F}\left(\mathbb{R}^{d}\right)$ of finite measures on $\mathbb{R}^{d}$, which quantifies how likely it is that a particular mass distribution occurs for the $m^{\text {th }}$ generation of a critical branching random walk in $n^{-1 / 2} \mathbb{Z}^{d}$. So far, we have rescaled generation $m$ to time $m / n$, space to $n^{-1 / 2} \mathbb{Z}^{d}$, and vertex mass has been set equal to $n^{-1}$. 


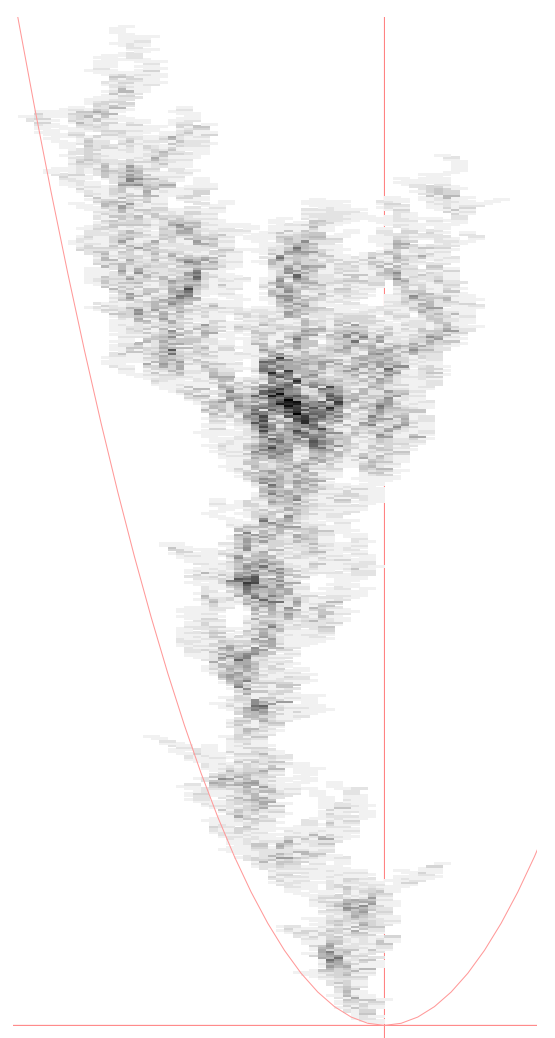

Fig. 17.1. The evolution of a mass distribution under the canonical measure, with time vertical and 1-dimensional space horizontal. Darker shading represents higher mass, and the parabola $t=x^{2}$ shows the spatial scaling.

It remains to scale the probability measure $\mathcal{R}_{n}^{(m / n)}$. When $m$ is of order $n$, the random measure $R_{n}^{(m / n)}$ is the zero measure on $\mathbb{R}^{d}$ with probability $1-O\left(n^{-1}\right)$, since the probability of survival for $m$ generations is order $m^{-1} \approx$ $n^{-1}$. To amplify the rare event of survival to $m$ generations, we consider the measure $n \mathcal{R}_{n}^{(m / n)}$ on $M_{F}\left(\mathbb{R}^{d}\right)$, which has total measure $n$. This measure places measure $n-O(1)$ on the zero measure on $\mathbb{R}^{d}$, and the remaining measure $O(1)$ on the nontrivial mass distributions due to the rare trees that survive for $m$ generations.

It is known that the limit of $n \mathcal{R}_{n}^{(\lfloor t n\rfloor / n)}$, as $n \rightarrow \infty$, consists of an infinite point mass on the zero measure on $\mathbb{R}^{d}$, plus a nontrivial finite measure $\mathcal{R}^{(t)}$ on $M_{0}\left(\mathbb{R}^{d}\right)$, the finite measures on $\mathbb{R}^{d}$ excluding the zero measure (see [177]). More precisely, there is a measure $\mathcal{R}^{(t)}$ on $M_{0}\left(\mathbb{R}^{d}\right)$ such that $n \mathcal{R}_{n}^{(\lfloor t n\rfloor / n)}$ converges weakly to $\mathcal{R}^{(t)}$ plus an infinite point mass on the zero measure on $\mathbb{R}^{d}$. In particular, for every bounded continuous function $f$ on $M_{0}\left(\mathbb{R}^{d}\right)$, 


$$
\lim _{n \rightarrow \infty} n \int_{M_{0}\left(\mathbb{R}^{d}\right)} f(R) \mathrm{d} \mathcal{R}_{n}^{(\lfloor t n\rfloor / n)}(R)=\int_{M_{0}\left(\mathbb{R}^{d}\right)} f(R) \mathrm{d} \mathcal{R}^{(t)}(R) .
$$

In other words, when normalized by $n$, the average of a function over the long-lived, random configurations of the discrete mass distribution defined by $\mathcal{R}_{n}^{(\lfloor t n\rfloor / n)}$ converges to a corresponding integral over the configurations of the continuous mass distribution defined by $\mathcal{R}^{(t)}$.

Moreover, stronger results [178, Section 7] assert the convergence of the scaling limit in the sense of convergence of measures on functions $R^{(\cdot)}$ from the time interval $(0, \infty)$ into $M_{F}\left(\mathbb{R}^{d}\right)$. The measure-valued path $R^{(\cdot)}$ represents the evolving mass distribution of the embedded critical tree. The canonical measure of super-Brownian motion, denoted $\mathbb{N}_{0}$, is a measure on measurevalued paths that gives the appropriate weight to $R^{(\cdot)}$. Fig. 17.1 illustrates the evolution.

\subsection{Moment Measures of the Canonical Measure}

Later we will state results showing convergence of critical oriented percolation and the critical contact process to the canonical measure, in the sense of convergence of the moment measures. This is a form of convergence of finitedimensional distributions. The results make use of explicit formulas for the moment measures of the canonical measure of SBM, and we now discuss these formulas.

By definition, the $l^{\text {th }}$ moment measure of the canonical measure $\mathbb{N}_{0}$ has Fourier transform

$$
\hat{M}_{\bar{t}}^{(l)}(\bar{k})=\mathbb{N}_{0}\left(\int_{\mathbb{R}^{d l}} \mathrm{~d} R^{\left(t_{1}\right)}\left(x_{1}\right) \cdots \mathrm{d} R^{\left(t_{l}\right)}\left(x_{l}\right) \prod_{j=1}^{l} \mathrm{e}^{\mathrm{i} k_{j} \cdot x_{j}}\right) .
$$

Here $\bar{k}=\left(k_{1}, \ldots, k_{l}\right)$ with each $k_{j} \in \mathbb{R}^{d}$, and $R^{(\cdot)}$ has distribution $\mathbb{N}_{0}$, so that each $R^{(t)}$ is a non-negative finite measure on $\mathbb{R}^{d}$. It is possible to guess the form of (17.3) by thinking about the approximating branching random walk. In this section, we explain the guess, and sketch a proof for the case of critical Poisson branching considered in Section 15.2.

According to the description of the canonical measure in Section 17.1, the discrete analogue of (17.3) is

$$
\hat{M}_{n, \bar{t}}^{(l)}(\bar{k})=\frac{1}{n^{l-1}} \hat{S}_{\lfloor n \bar{t}\rfloor}^{(l+1)}(\bar{k} / \sqrt{n}),
$$

where

$$
S_{\bar{m}}^{(l+1)}(\bar{x})=\sum_{(T, \varphi)} \mathbb{P}(T, \varphi) \sum_{\substack{i_{1}, \ldots, i_{l} \in T \\\left|i_{j}\right|=m_{j} \forall j}} \prod_{j=1}^{l} I\left[\varphi\left(i_{j}\right)=x_{j}\right]
$$


The factors involving $n$ in (17.4) arise as follows. The scaling of $\bar{k}$ by $n^{-1 / 2}$ corresponds to scaling the lattice spacing, the scaling of $\bar{t}$ by $n$ is the temporal scaling, a factor $n^{-l}$ is due to mass $n^{-1}$ at each of the $l$ vertices $x_{1}, \ldots, x_{l}$, and an additional factor $n$ changes $n^{-l}$ to $n^{-(l-1)}$ to rescale probability.

Recall the notions of shape, skeleton and compatibility from Section 15.2. Suppose that $(\sigma ; \vec{y}, \vec{s})$ is compatible with $(T, \varphi, \bar{\imath})$ contributing to the sum in (17.5), where $\sigma \in \Sigma_{l+1}$, and where, as in Section 15.2, we write e.g. $\vec{s}=\left(s_{1}, \ldots, s_{2 l-1}\right)$ for $(2 l-1)$-component vectors $(l$ corresponds to $r-1)$. Compatibility forces relationships between $\vec{s}$ and $\bar{m}$ and between $\vec{y}$ and $\bar{x}$. To be specific about these relationships, we make the following definitions.

Given a shape $\sigma \in \Sigma_{l+1}$, for each vertex $j$ of degree 1 in $\sigma$, other than vertex 0 , we let $\omega_{j}$ be the set of edges in $\sigma$ on the path from 0 to $j(j=1, \ldots, l)$. Given $\vec{s} \in \mathbb{R}_{+}^{2 l-1}$, we define the $j^{\text {th }}$ component $\bar{m}_{j}(\sigma, \vec{s})$ of $\bar{m}(\sigma, \vec{s}) \in \mathbb{R}_{+}^{l}$ by

$$
\bar{m}_{j}(\sigma, \vec{s})=\sum_{i \in \omega_{j}} s_{i}
$$

and compatibility requires (17.6). For $t_{i}>0$ we also define an $(l-1)$ dimensional subset $J_{\bar{t}}(\sigma)$ of $\mathbb{R}_{+}^{2 l-1}$ by

$$
J_{\bar{t}}(\sigma)=\{\vec{s}: \bar{m}(\sigma, \vec{s})=\bar{t}\} .
$$

For example, for $l=2$, there is a unique shape $\sigma$ and we have simply

$$
J_{\bar{t}}(\sigma)=\left\{\left(s, t_{1}-s, t_{2}-s\right): s \in\left[0, t_{1} \wedge t_{2}\right]\right\} .
$$

The relation between $\bar{m}$ and $\vec{s}$ has a natural counterpart for $\bar{x}$ and $\vec{y}$. This translates into a dual relation for the Fourier variable $\bar{k}$, which we recall from Section 16.3. Given a shape $\sigma \in \Sigma_{l+1}$ and $\bar{k}=\left(k_{1} \ldots, k_{l}\right) \in \mathbb{R}^{d l}$, we define $\vec{k}(\sigma) \in \mathbb{R}^{(2 l-1) d}$ by setting the $i^{\text {th }}$ component $\vec{k}_{i}(\sigma) \in \mathbb{R}^{d}(i=1, \ldots, 2 l-1)$ of $\vec{k}(\sigma)$ to be

$$
\vec{k}_{i}(\sigma)=\sum_{j=1}^{l} k_{j} I\left[i \in \omega_{j}\right]
$$

where, on the right hand side, $k_{j}$ denotes the $j^{\text {th }}$ component of $\bar{k}$ and $I$ is an indicator function.

For $l \geq 3$, there are redundancies in replacing the sum over $(T, \varphi, \bar{l})$ in $S_{\bar{m}}^{(l+1)}(\bar{x})$ by an appropriate sum over $(\sigma ; \vec{y}, \vec{s})$, as discussed in the proof of Theorem 16.5. However, such overcounting arises from degenerate configurations with at least one $s_{j}$ equal to zero, and it can be shown that these are lower order and do not contribute to the limit. Therefore, in place of the right hand side of (17.4), we consider instead

$$
\sum_{\sigma \in \Sigma_{l+1}} \frac{1}{n^{l-1}} \sum_{\vec{s} \in J_{\lfloor n \bar{t}\rfloor}(\sigma) \cap \mathbb{Z}_{+}^{l-1}} \sum_{N=0}^{\infty} \hat{b}_{N}^{(l+1)}\left(\sigma ; \vec{k}(\sigma) n^{-1 / 2}, \vec{s}\right)
$$


(where $\hat{b}_{N}$ is the Fourier transform of (15.23) with respect to $\vec{y}$ ), which can be shown to have the same limit as (17.4) when $n \rightarrow \infty$. By Theorem 15.4, the quantity $\sum_{N=0}^{\infty} b_{N}^{(l+1)}\left(\sigma ; \vec{k} n^{-1 / 2}, \vec{s}\right)$ is the coefficient of $\prod_{j=1}^{2 l-1} \zeta_{j}^{s_{j}}$ in the generating function

$$
\prod_{j=1}^{2 l-1} \frac{1}{1-\zeta_{j} \hat{D}\left(k_{j} n^{-1 / 2}\right)} .
$$

As in (16.18), this coefficient converges to a product of Gaussians. The set $J_{\lfloor n \bar{t}\rfloor}(\sigma)$ is $(l-1)$-dimensional, and the factor $n^{-(l-1)}$ converts the sum into a Riemann sum. It is therefore entirely plausible, and it can be proven, that if $t_{i}>0$ for all $i$ then

$$
\lim _{n \rightarrow \infty} \hat{M}_{n, \bar{t}}^{(l)}(\bar{k})= \begin{cases}\mathrm{e}^{-|k|^{2} t / 2 d} & (l=1), \\ \sum_{\sigma \in \Sigma_{l+1}} \int_{J_{\bar{t}}(\sigma)} \mathrm{d} \vec{s} \prod_{j=1}^{2 l-1} \mathrm{e}^{-\left|k_{j}(\sigma)\right|^{2} s_{j} / 2 d} & (l \geq 2) .\end{cases}
$$

A different but related and more general proof of (17.12) is discussed in [109]. The right hand side of (17.12) is equal to $\hat{M}_{\bar{t}}^{(l)}(\bar{k})$ of $(17.3)$. The latter fact is essentially [2, Theorem 3.1] (see also [69] and [152, Proposition IV.2.(ii)]).

Exercise 17.1. Fill in the details missing in the above discussion to give a complete proof of (17.12).

Explicitly, for $l=2$,

$$
\hat{M}_{t_{1}, t_{2}}^{(2)}\left(k_{1}, k_{2}\right)=\int_{0}^{t_{1} \wedge t_{2}} \mathrm{~d} s \mathrm{e}^{-\left|k_{1}+k_{2}\right|^{2} s / 2 d} \mathrm{e}^{-\left|k_{1}\right|^{2}\left(t_{1}-s\right) / 2 d} \mathrm{e}^{-\left|k_{2}\right|^{2}\left(t_{2}-s\right) / 2 d} .
$$

In $x$-space, recalling the definition of the Gaussian density $p_{t}(x)$ in (6.8), this is equivalent to the density

$$
M_{t_{1}, t_{2}}^{(2)}\left(x_{1}, x_{2}\right)=\int_{0}^{t_{1} \wedge t_{2}} \mathrm{~d} s \int_{\mathbb{R}^{d}} \mathrm{~d} y p_{s}(y) p_{t_{1}-s}\left(x_{1}-y\right) p_{t_{2}-s}\left(x_{2}-y\right) .
$$

This last formula clearly shows the branching structure. Mass arrives at $x_{1}$ at time $t_{1}$ and at $x_{2}$ at time $t_{2}$ via a Brownian path that leaves the origin and splits into two at a time $s$ chosen uniformly from $\left[0, t_{1} \wedge t_{2}\right]$, with the two particles then continuing to $x_{1}$ and $x_{2}$ at their appointed times.

Exercise 17.2. Starting with the right hand side of (17.12) as an expression for the Fourier transform $\hat{M}_{\bar{t}}^{(l)}(\bar{k})$ of the moment measures, prove the recursion relation

$$
\hat{M}_{\bar{t}}^{(l)}(\bar{k})=\int_{0}^{\underline{t}} d t \hat{M}_{t}^{(1)}\left(k_{1}+\cdots+k_{l}\right) \sum_{I \subset J_{1}:|I| \geq 1} \hat{M}_{\bar{t}_{I}-t}^{(i)}\left(\bar{k}_{I}\right) \hat{M}_{\bar{t}_{J \backslash I}-t}^{(l-i)}\left(\bar{k}_{J \backslash I}\right)
$$

for $l \geq 2$, where $i=|I|, J=\{1, \ldots, l\}, J_{1}=J \backslash\{1\}, \underline{t}=\min _{i} t_{i}, \bar{t}_{I}$ denotes the vector consisting of the components $t_{i}$ of $\bar{t}$ with $i \in I$, and $\bar{t}_{I}-t$ denotes subtraction of $t$ from each component of $\bar{t}_{I}$. 
A solution to a slightly weakened version of the following exercise can be found in [114].

Exercise 17.3. Prove that for $l \geq 1, t_{1} \geq s=\max \left\{t_{2}, \ldots, t_{l}\right\}$ and $k_{j} \in \mathbb{R}^{d}$,

$$
\hat{M}_{t_{1}, t_{2}, \ldots, t_{l}}^{(l)}\left(0, k_{2}, \ldots, k_{l}\right)=\hat{M}_{s, t_{2}, \ldots, t_{l}}^{(l)}\left(0, k_{2}, \ldots, k_{l}\right) .
$$

A solution to the following exercise can be found in [114].

Exercise 17.4. Prove that for $l \geq 1$,

$$
\hat{M}_{t, \ldots, t}^{(l)}(\overrightarrow{0})=t^{l-1} 2^{-l+1} l !
$$

\subsection{Critical Oriented Percolation and SBM}

For $r \geq 2$, the oriented percolation $r$-point functions are defined by

$$
\tau_{p, \bar{n}}^{(r)}(\bar{x})=\mathbb{P}_{p}\left((0,0) \rightarrow\left(x_{i}, n_{i}\right) \text { for each } i=1, \ldots, r-1\right) .
$$

The event on the right hand side makes no statement about the occurrence of $\left(x_{i}, n_{i}\right) \rightarrow\left(x_{j}, n_{j}\right)$ for any $i \neq j$. The following theorem is proved in [121]. In its statement, the constants $A$ and $v$ are the same as those appearing in Theorem 12.3.

Theorem 17.5. Consider the spread-out model of oriented percolation. Let $d>4, p=p_{c}, \delta \in\left(0,1 \wedge \frac{d-4}{2}\right), r \geq 2, \bar{t}=\left(t_{1}, \ldots, t_{r-1}\right) \in(0, \infty)^{r-1}$, and $\bar{k}=\left(k_{1}, \ldots, k_{r-1}\right) \in \mathbb{R}^{(r-1) d}$. There is a constant $V=V(d, L)$, with $|V-1| \leq C L^{-d}$, and an $L_{0}=L_{0}(d)$ (independent of $r$ ) such that for $L \geq L_{0}$,

$$
\hat{\tau}_{p_{c},\lfloor n \bar{t}\rfloor}^{(r)}(\bar{k} / \sqrt{v n})=n^{r-2} V^{r-2} A^{2 r-3}\left[\hat{M}_{\bar{t}}^{(r-1)}(\bar{k})+O\left(n^{-\delta}\right)\right],
$$

with the error estimate uniform in $\bar{k}$ in a bounded subset of $\mathbb{R}^{(r-1) d}$ (but not in $L$ or the $t_{j}$ ).

The theorem is proved using induction on $r$. The induction is started at $r=2$ with Theorem 12.3, which gives control of the critical two-point function (and which was proved using induction on $n$ ). The induction on $r$ is advanced using a second expansion for the $r$-point functions for $r \geq 3$, which goes beyond the expansion methods of Section 13.

For $r=3$, the main term on the right hand side of (17.19) reduces to

$$
n V A^{3} \int_{0}^{t_{1} \wedge t_{2}} \mathrm{~d} s \mathrm{e}^{-\left|k_{1}+k_{2}\right|^{2} s / 2 d} \mathrm{e}^{-\left|k_{1}\right|^{2}\left(t_{1}-s\right) / 2 d} \mathrm{e}^{-\left|k_{2}\right|^{2}\left(t_{2}-s\right) / 2 d} .
$$

Equation (17.20) can be interpreted as indicating that a cluster connecting the origin to $\left(x_{1},\left\lfloor n t_{1}\right\rfloor\right)$ and $\left(x_{2},\left\lfloor n t_{2}\right\rfloor\right)$, with the $x_{i}$ of order $n^{1 / 2}$, can be 
considered to decompose into a product of three independent two-point functions joined together at a branch point. Each two-point function gives rise to a Gaussian, together with a factor $A$, according to Theorem 12.3(a). This decomposition into independent two-point functions is not exact, but is compensated by the vertex factor $V$ associated with the branch point. The integral with respect to $s$ corresponds to a sum over possible temporal locations of the branch point, with the additional factor $n$ accounting for the change from a sum to an integral.

Similar considerations apply to (17.19), with additional structure due to the proliferation of shapes. There are $r-2$ branch points in the general case, each contributing $n V$, and $2 r-3$ two-point functions, each contributing $A$ times a Gaussian. The integral over $J_{\bar{t}}(\sigma)$ corresponds to a sum over time intervals between the various branch points, and is constrained so that the shape's leaves are specified by the times $\lfloor n \bar{t}\rfloor$.

The tree graph bounds (derivations in $[12,85]$ extend easily to the oriented setting), together with the bound on the two-point function of Theorem 15.2(a), immediately imply that the left hand side of (17.19) is bounded above by a multiple of $n^{r-2}$. By (17.19), this elementary upper bound gives the correct power of $n$, above the upper critical dimension.

Theorem 17.5 can be rephrased to say that, under its hypotheses, the moment measures of rescaled critical oriented percolation converge to those of the canonical measure of super-Brownian motion. We now make this interpretation more explicit.

First, for $t \in(0, \infty)$, we define $X_{n, t}$ to be the discrete finite random measure on $\mathbb{R}^{d}$ placing mass $\left(A^{2} V n\right)^{-1}$ at each vertex at time $\lfloor n t\rfloor$ in $(v n)^{-1 / 2} C(0,0)$. The characteristic function of the $l^{\text {th }}$ moment measure of the measure-valued process $X_{n}$. is defined as in (17.3) and is given by

$$
\hat{N}_{n, \bar{t}}^{(l)}(\bar{k})=\left(A^{2} V n\right)^{-l} \hat{\tau}_{\lfloor n \bar{t}\rfloor}^{(l+1)}(\bar{k} / \sqrt{v n}) .
$$

It then follows immediately from Theorems 15.2 and 17.5 that, under their hypotheses, for all $l \geq 1$,

$$
\lim _{n \rightarrow \infty} A V n \hat{N}_{n, \bar{t}}^{(l)}(\bar{k})=\hat{M}_{\bar{t}}^{(l)}(\bar{k}) .
$$

This can be regarded as a statement that $A V n \mathbb{P}\left(\left\{X_{n, t}\right\}_{t>0} \in \cdot\right)$ converges to $\mathbb{N}_{0}$ in the sense of convergence of finite-dimensional distributions.

This shows that spread-out critical oriented percolation and critical branching random walk have the same scaling limit, for $d>4$ (compare [177, Theorem II.7.3(a)]). A crucial difference between oriented percolation and branching random walk is that particles can coexist at the same vertex for the latter, but not for the former.

The above gives a statement of convergence of finite-dimensional distributions. To prove weak convergence, as a measure-valued process, of rescaled 
spread-out oriented percolation for $d>4$ to the canonical measure of superBrownian motion, it would be necessary also to prove tightness. Currently, this is an open problem.

By Theorem 12.7, if the survival probability is as expected asymptotic to $(B n)^{-1}$ then it must be the case that $B=A V / 2$, which in turn implies that the factor $A V n$ in (17.22) corresponds asymptotically to twice the reciprocal of the survival probability.

The incipient infinite cluster of spread-out oriented percolation in dimensions $d+1>4+1$ was discussed in Section 12.4. In [121], the natural conjecture was formulated that the scaling limit of the incipient infinite cluster for oriented percolation in dimensions $d+1>4+1$ is the canonical measure of super-Brownian motion, conditioned on survival for all time. The proof makes use of the IIC $r$-point functions, defined by

$$
\rho_{\bar{m}}^{(r)}(\bar{y})=\mathbb{P}_{\infty}\left((0,0) \rightarrow\left(y_{i}, m_{i}\right) \text { for each } i=1, \ldots, r-1\right),
$$

where $\mathbb{P}_{\infty}$ is the measure of Theorem 12.5. It is proved in [114] that the scaling limit of the IIC $r$-point functions is given by

$$
\lim _{m \rightarrow \infty} \frac{1}{\left(m A^{2} V\right)^{r-1}} \hat{\rho}_{\lfloor m \bar{t}\rfloor}^{(r)}(\bar{k} / \sqrt{v m})=\hat{M}_{1, \bar{t}}^{(r)}(0, \bar{k}),
$$

for all $r \geq 2, \bar{t}=\left(t_{1}, \ldots, t_{r-1}\right) \in(0,1]^{r-1}$ and $\bar{k} \in \mathbb{R}^{d(r-1)}$, and it is pointed out in [109] that this proves the conjecture at the level of convergence of finite-dimensional distributions.

Solutions to the following two exercises can be found in [114]. Each makes use of the following extension of Theorem 17.5 that was proved in [121]. Let $m$ denote the second largest component of $\bar{n}=\left(n_{1}, \ldots, n_{r-1}\right)$. Then [121, (2.52)] states that for each $r \geq 3$,

$$
\hat{\tau}_{\bar{n}}^{(r)}(\bar{k} / \sqrt{v n})=n^{r-2} V^{r-2} A^{2 r-3}\left[\hat{M}_{\bar{n} / n}^{(r-1)}(\bar{k})+\mathcal{O}\left((m+1)^{-\delta}\right)\right]
$$

holds uniformly in $n \geq m$.

Exercise 17.6. Prove Theorem 12.7(a), using (17.25), Exercises 17.3 and 17.4, and

$$
\mathbb{E}_{\infty}\left[N_{m}^{l}\right]=\lim _{n \rightarrow \infty} \frac{\hat{\tau}_{n, m, \ldots, m}^{(l+2)}(0)}{\hat{\tau}_{n}^{(2)}(0)} .
$$

Exercise 17.7. Prove (17.24) for all $r \geq 2$. Use Exercise 17.3 and (17.25).

\subsection{The Critical Contact Process and SBM}

A version of Theorem 17.5 is proven in [117] for the contact process. This shows that in dimensions $d>4$ the spread-out contact process with $L$ sufficiently large converges to the canonical measure of super-Brownian motion in the 
sense of convergence of finite-dimensional distributions. The proof uses the approximation by oriented percolation discussed in Section 14.2. In addition, a similar result is obtained in [117] for dimensions $1 \leq d \leq 4$, for the model with $L=L_{1} T^{b}$ with parameters as in Theorem 14.3. In particular, the assumption on the exponent $b$ is that $b>\frac{4-d}{2 d}$, as in (14.24).

This should be compared with the results of [68], who proved convergence to super-Brownian motion for the model of the contact process with $L$ proportional to $T$ for $d>2$ and proportional to $(T \log T)^{1 / 2}$ for $d=2$. This requires faster growth in $T$ for $d>2$ but is sharper for $d=2$. The results of [68] use completely different methods than the lace expansion methods discussed in these lecture notes. In particular, they work entirely with continuous time and do not use an approximation by oriented percolation. The convergence statements of [68] are stronger than those of [117] in the sense that they also prove tightness and thus go beyond the convergence of moment measures. In addition, their results are not formulated in terms of the canonical measure, but rather prove convergence to super-Brownian motion with an arbitrary finite measure as initial condition. On the other hand, their assumption that $b=1$ in dimensions $d>4$ is considerably stronger than the assumption in [117] that $L$ is large but finite for dimensions $d>4$.

\subsection{Lattice Trees and SBM}

Corollary 16.8 shows that in dimensions $d>8$ spread-out lattice trees converge to ISE when appropriately rescaled. In that scaling, we considered lattice trees with exactly $n$ bonds, mass $(n+1)^{-1}$ was placed at each vertex, space was rescaled by a multiple of $n^{-1 / 4}$, and the limit $n \rightarrow \infty$ was taken. Now we consider instead the scaling of Section 17.1, appropriate to give the canonical measure of SBM as limit.

For this, we need a time variable for lattice trees. The time variable is graph distance from the origin, i.e., vertices at "time" $m$ are those at graph distance $m$ from the origin in the lattice tree. Let $|x|_{\mathrm{g}}$ denote the graph distance of $x$, and for $r \geq 2, \bar{m}=\left(m_{1}, \ldots, m_{r-1}\right)$, and $\bar{x}=\left(x_{1}, \ldots, x_{r-1}\right)$ such that $\left|x_{i}\right|_{\mathrm{g}}=m_{i}$ for all $i$, define

$$
t_{\bar{m}}^{(r)}(\bar{x})=\sum_{T: T \ni 0, x_{1}, \ldots, x_{r-1}} z_{c}^{|T|} .
$$

Note that the activity $z$ dual to the size of the lattice tree has been set equal to its critical value $z_{c}$, and that lattice trees of arbitrary size contribute to (17.27).

We then rescale $\bar{x}$ by $n^{-1 / 2}$ and rescale $\bar{m}$ by $n^{-1}$. Under this scaling, the analogue of (17.19) is proved in [125] to hold for sufficiently spread-out lattice trees in dimensions $d>8$. The proof uses an adaptation of the induction method of [120] for the two-point function, and then applies induction on $r$ for the $r$-point functions with $r \geq 3$. The induction on $r$ is advanced using the 
lace expansion on a tree [124] in place of the more difficult double expansion used in $[62,99]$. 


\section{References}

1. J. Adler, Y. Meir, A. Aharony, and A.B. Harris. Series study of percolation moments in general dimension. Phys. Rev. B, 41:9183-9206, (1990).

2. R.J. Adler. Superprocess local and intersection local times and their corresponding particle pictures. In E. Çinlar, K.L. Chung, and M.J. Sharpe, editors, Seminar on Stochastic Processes 1992, pages 1-42, Boston, (1993). Birkhäuser.

3. M. Aizenman. Geometric analysis of $\varphi^{4}$ fields and Ising models, Parts I and II. Commun. Math. Phys., 86:1-48, (1982).

4. M. Aizenman. The geometry of critical percolation and conformal invariance. In Hao Bai-Lin, editor, STATPHYS 19, Proceedings Xiamen 1995. World Scientific, (1996).

5. M. Aizenman. On the number of incipient spanning clusters. Nucl. Phys. B [FS], 485:551-582, (1997).

6. M. Aizenman. Scaling limit for the incipient spanning clusters. In Mathematics of multiscale materials, pages 1-24, IMA Vol. Math. Appl., 99. Springer, New York, (1998).

7. M. Aizenman and D.J. Barsky. Sharpness of the phase transition in percolation models. Commun. Math. Phys., 108:489-526, (1987).

8. M. Aizenman and A. Burchard. Hölder regularity and dimension bounds for random curves. Duke Math. J., 99:419-453, (1999).

9. M. Aizenman and R. Fernández. On the critical behavior of the magnetization in high dimensional Ising models. J. Stat. Phys., 44:393-454, (1986).

10. M. Aizenman and R. Fernández. Critical exponents for long-range interactions. Lett. Math. Phys., 16:39-49, (1988).

11. M. Aizenman, H. Kesten and C.M. Newman. Uniqueness of the infinite cluster and continuity of connectivity functions for short and long range percolation. Commun. Math. Phys., 111:505-531, (1987).

12. M. Aizenman and C.M. Newman. Tree graph inequalities and critical behavior in percolation models. J. Stat. Phys., 36:107-143, (1984).

13. M. Ajtai, J. Komlós, and E. Szemerédi. Largest random component of a $k$-cube. Combinatorica, 2:1-7, (1982).

14. D. Aldous. The continuum random tree III. Ann. Probab., 21:248-289, (1993).

15. D. Aldous. Tree-based models for random distribution of mass. J. Stat. Phys., 73:625-641, (1993).

16. S.E. Alm and S. Janson. Random self-avoiding walks on one-dimensional lattices. Commun. Statist.-Stochastic Models, 6:169-212, (1990).

17. N. Alon, I. Benjamini, and A. Stacey. Percolation on finite graphs and isoperimetric inequalities. Ann. Probab., 32:1727-1745, (2004).

18. M.N. Barber and B.W. Ninham. Random and Restricted Walks: Theory and Applications. Gordon and Breach, New York, (1970).

19. M.T. Barlow and E.A. Perkins. On the filtration of historical Brownian motion. Ann. Probab., 22:1273-1294, (1994).

20. D.J. Barsky and M. Aizenman. Percolation critical exponents under the triangle condition. Ann. Probab., 19:1520-1536, (1991).

21. D.J. Barsky and C.C. Wu. Critical exponents for the contact process under the triangle condition. J. Stat. Phys., 91:95-124, (1998).

22. C. Bezuidenhout and G. Grimmett. The critical contact process dies out. Ann. Probab., 18:1462-1482, (1990). 
23. C. Bezuidenhout and G. Grimmett. Exponential decay for subcritical contact and percolation processes. Ann. Probab., 19:984-1009, (1991).

24. P. Billingsley. Convergence of Probability Measures. John Wiley and Sons, New York, (1968).

25. B. Bollobás. The evolution of random graphs. Trans. Amer. Math. Soc., 286:257-274, (1984).

26. B. Bollobás. Random Graphs. Cambridge University Press, Cambridge, 2nd edition, (2001).

27. B. Bollobás and Y. Kohayakawa. Percolation in high dimensions. Europ. J. Combinatorics, 15:113-125, (1994).

28. B. Bollobás, Y. Kohayakawa, and T. Łuczak. The evolution of random subgraphs of the cube. Random Struct. Alg., 3:55-90, (1992).

29. E. Bolthausen and C. Ritzmann. A central limit theorem for convolution equations and weakly self-avoiding walks. To appear in Ann. Probab.

30. C. Borgs, J.T. Chayes, R. van der Hofstad, and G. Slade. Mean-field lattice trees. Ann. Combinatorics, 3:205-221, (1999).

31. C. Borgs, J.T. Chayes, R. van der Hofstad, G. Slade, and J. Spencer. Random subgraphs of finite graphs: I. The scaling window under the triangle condition. Random Struct. Alg., 27:137-184, (2005).

32. C. Borgs, J.T. Chayes, R. van der Hofstad, G. Slade, and J. Spencer. Random subgraphs of finite graphs: II. The lace expansion and the triangle condition. To appear in Ann. Probab.

33. C. Borgs, J.T. Chayes, R. van der Hofstad, G. Slade, and J. Spencer. Random subgraphs of finite graphs: III. The phase transition for the $n$-cube. To appear in Combinatorica.

34. C. Borgs, J.T. Chayes, H. Kesten, and J. Spencer. Uniform boundedness of critical crossing probabilities implies hyperscaling. Random Struct. Alg., 15:368413, (1999).

35. C. Borgs, J.T. Chayes, H. Kesten, and J. Spencer. The birth of the infinite cluster: finite-size scaling in percolation. Commun. Math. Phys., 224:153-204, (2001).

36. A. Bovier, J. Fröhlich, and U. Glaus. Branched polymers and dimensional reduction. In K. Osterwalder and R. Stora, editors, Critical Phenomena, Random Systems, Gauge Theories, Amsterdam, (1986). North-Holland.

37. M. Bramson, J.T. Cox, and J-F. Le Gall. Super-Brownian limits of voter model clusters. Ann. Probab., 29:1001-1032, (2001).

38. E. Brézin, J.C. Le Guillou, and J. Zinn-Justin. Approach to scaling in renormalized perturbation theory. Phys. Rev. D, 8:2418-2430, (1973).

39. D. Brydges, S.N. Evans, and J.Z. Imbrie. Self-avoiding walk on a hierarchical lattice in four dimensions. Ann. Probab., 20:82-124, (1992).

40. D.C. Brydges. A short course on cluster expansions. In K. Osterwalder and R. Stora, editors, Critical Phenomena, Random Systems, Gauge Theories, Amsterdam, (1986). North-Holland. Les Houches 1984.

41. D.C. Brydges and J.Z. Imbrie. Branched polymers and dimensional reduction. Ann. Math., 158:1019-1039, (2003).

42. D.C. Brydges and J.Z. Imbrie. Dimensional reduction formulas for branched polymer correlation functions. J. Stat. Phys., 110:503-518, (2003).

43. D.C. Brydges and J.Z. Imbrie. End to end distance from the Green's function for a hierarchical self-avoiding walk in four dimensions. Commun. Math. Phys., 239:523-547, (2003). 
44. D.C. Brydges and J.Z. Imbrie. Green's function for a hierarchical self-avoiding walk in four dimensions. Commun. Math. Phys., 239:549-584, (2003).

45. D.C. Brydges and T. Spencer. Self-avoiding walk in 5 or more dimensions. Commun. Math. Phys., 97:125-148, (1985).

46. R.M. Burton and M. Keane. Density and uniqueness in percolation. Commun. Math. Phys., 121:501-505, (1989).

47. S. Caracciolo, G. Parisi, and A. Pelissetto. Random walks with short-range interaction and mean-field behavior. J. Stat. Phys., 77:519-543, (1994).

48. J.T. Chayes and L. Chayes. Ornstein-Zernike behavior for self-avoiding walks at all noncritical temperatures. Commun. Math. Phys., 105:221-238, (1986).

49. J.T. Chayes and L. Chayes. Percolation and random media. In K. Osterwalder and R. Stora, editors, Critical Phenomena, Random Systems, Gauge Theories, Amsterdam, (1986). North-Holland. Les Houches 1984.

50. J.T. Chayes and L. Chayes. On the upper critical dimension of Bernoulli percolation. Commun. Math. Phys., 113:27-48, (1987).

51. J.T. Chayes, L. Chayes, and R. Durrett. Inhomogeneous percolation problems and incipient infinite clusters. J. Phys. A: Math. Gen., 20:1521-1530, (1987).

52. Y. Cheng. Long range self-avoiding random walks above critical dimension. PhD thesis, Temple University, (2000).

53. N. Clisby, R. Liang and G. Slade. Self-avoiding walk enumeration via the lace expansion. In preparation.

54. A.R. Conway, I.G. Enting, and A.J. Guttmann. Algebraic techniques for enumerating self-avoiding walks on the square lattice. J. Phys. A: Math. Gen., 26:1519-1534, (1993).

55. A.R. Conway and A.J. Guttmann. Lower bound on the connective constant for square lattice self-avoiding walks. J. Phys. A: Math. Gen., 26:3719-3724, (1993).

56. R.M. Corless, G.H. Gonnet, D.E.G. Hare, D.J. Jeffrey, and D.E. Knuth. On the Lambert $W$ function. Adv. Comput. Math., 5:329-359, (1996).

57. J.T. Cox and R. Durrett. Oriented percolation in dimensions $d \geq 4$ : bounds and asymptotic formulas. Math. Proc. Cambridge Philos. Soc., 93:151-162, (1983).

58. J.T. Cox, R. Durrett, and E.A. Perkins. Rescaled voter models converge to super-Brownian motion. Ann. Probab., 28:185-234, (2000).

59. T. Cox, R. Durrett, and E.A. Perkins. Rescaled particle systems converging to super-Brownian motion. In M. Bramson and R. Durrett, editors, Perplexing Problems in Probability: Festschrift in Honor of Harry Kesten, Basel, (1999). Birkhäuser.

60. D.A. Dawson. Measure-valued Markov processes. In Ecole d'Eté de Probabilités de Saint-Flour 1991. Lecture Notes in Mathematics \#1541, Berlin, (1993). Springer.

61. E. Derbez and G. Slade. Lattice trees and super-Brownian motion. Canad. Math. Bull., 40:19-38, (1997).

62. E. Derbez and G. Slade. The scaling limit of lattice trees in high dimensions. Commun. Math. Phys., 193:69-104, (1998).

63. C. Domb and M.E. Fisher. On random walks with restricted reversals. Proc. Camb. Phil. Soc., 54:48-59, (1958).

64. C. Domb and G.S. Joyce. Cluster expansion for a polymer chain. J. Phys. C: Solid State Phys., 5:956-976, (1972). 
65. B. Duplantier. Polymer chains in four dimensions. Nuclear Physics B, $\mathbf{2 7 5}$ [FS17]:319-355, (1986).

66. B. Duplantier. Statistical mechanics of polymer networks of any topology. $J$. Stat. Phys., 54:581-680, (1989).

67. B. Duplantier. Renormalization and conformal invariance for polymers. In H. van Beijeren, editor, Fundamental Problems in Statistical Mechanics VII, pages 171-223, Amsterdam, (1990). Elsevier Science Publishers B.V.

68. R. Durrett and E.A. Perkins. Rescaled contact processes converge to superBrownian motion in two or more dimensions. Probab. Th. Rel. Fields, 114:309 399, (1999).

69. E.B. Dynkin. Representation for functionals of superprocesses by multiple stochastic integrals, with applications to self-intersection local times. Astérisque, 157-158:147-171, (1988).

70. I.G. Enting and A.J. Guttmann. Polygons on the honeycomb lattice. J. Phys. A: Math. Gen., 22:1371-1384, (1989).

71. P. Erdős and A. Rényi. On the evolution of random graphs. Magyar Tud. Akad. Mat. Kutató Int. Közl., 5:17-61, (1960).

72. M.H. Ernst. Random walks with short memory. J. Stat. Phys., 53:191-201, (1988).

73. R. Fernández, J. Fröhlich, and A.D. Sokal. Random Walks, Critical Phenomena, and Triviality in Quantum Field Theory. Springer, Berlin, (1992).

74. M.E. Fisher and D.S. Gaunt. Ising model and self-avoiding walks on hypercubical lattices and "high-density" expansions. Phys. Rev., 133:A224-A239, (1964).

75. P. Flajolet and A. Odlyzko. Singularity analysis of generating functions. SIAM J. Disc. Math., 3:216-240, (1990).

76. J. Fröhlich. On the triviality of $\varphi_{d}^{4}$ theories and the approach to the critical point in $d \geq 4$ dimensions. Nucl. Phys., B200 [FS4]:281-296, (1982).

77. D.S. Gaunt. 1/d expansions for critical amplitudes. J. Phys. A: Math. Gen., 19:L149-L153, (1986).

78. D.S. Gaunt and H. Ruskin. Bond percolation processes in $d$ dimensions. $J$. Phys. A: Math. Gen., 11:1369-1380, (1978).

79. P.G. de Gennes. Exponents for the excluded volume problem as derived by the Wilson method. Phys. Lett., A38:339-340, (1972).

80. P.G. de Gennes. Scaling Concepts in Polymer Physics. Cornell University Press, Ithaca, (1979).

81. S. Golowich and J.Z. Imbrie. A new approach to the long-time behavior of self-avoiding random walks. Ann. Phys., 217:142-169, (1992).

82. D.M. Gordon. Percolation in high dimensions. J. London Math. Soc. (2), 44:373-384, (1991).

83. I.S. Gradshteyn and I.M. Ryzhik. Table of Integrals, Series and Products. Academic Press, New York, 4th edition, (1965).

84. A. Greven and F. den Hollander. A variational characterization of the speed of a one-dimensional self-repellent random walk. Ann. Appl. Probab., 3:10671099, (1993).

85. G. Grimmett. Percolation. Springer, Berlin, 2nd edition, (1999).

86. G. Grimmett and P. Hiemer. Directed percolation and random walk. In V. Sidoravicius, editor, In and Out of Equilibrium, pages 273-297. Birkhäuser, Boston, (2002). 
87. G. Grimmett and D.R. Stirzaker. Probability and Random Processes. Oxford University Press, Oxford, 3rd edition, (2001).

88. J.M. Hammersley and D.J.A. Welsh. Further results on the rate of convergence to the connective constant of the hypercubical lattice. Quart. J. Math. Oxford, (2), 13:108-110, (1962).

89. T. Hara. Mean field critical behaviour for correlation length for percolation in high dimensions. Prob. Th. and Rel. Fields, 86:337-385, (1990).

90. T. Hara. Decay of correlations in nearest-neighbour self-avoiding walk, percolation, lattice trees and animals. Preprint, (2005).

91. T. Hara, R. van der Hofstad, and G. Slade. Critical two-point functions and the lace expansion for spread-out high-dimensional percolation and related models. Ann. Probab., 31:349-408, (2003).

92. T. Hara and G. Slade. The mean-field critical behaviour of percolation in high dimensions. In B. Simon, A. Truman, and I.M. Davies, editors, Proceedings of the IXth International Congress on Mathematical Physics, pages 450-453, Bristol and New York, (1989). Adam Hilger.

93. T. Hara and G. Slade. The triangle condition for percolation. Bull. A.M.S., 21:269-273, (1989).

94. T. Hara and G. Slade. Mean-field critical behaviour for percolation in high dimensions. Commun. Math. Phys., 128:333-391, (1990).

95. T. Hara and G. Slade. On the upper critical dimension of lattice trees and lattice animals. J. Stat. Phys., 59:1469-1510, (1990).

96. T. Hara and G. Slade. Critical behaviour of self-avoiding walk in five or more dimensions. Bull. A.M.S., 25:417-423, (1991).

97. T. Hara and G. Slade. Self-avoiding walk in five or more dimensions. I. The critical behaviour. Commun. Math. Phys., 147:101-136, (1992).

98. T. Hara and G. Slade. The lace expansion for self-avoiding walk in five or more dimensions. Reviews in Math. Phys., 4:235-327, (1992).

99. T. Hara and G. Slade. The number and size of branched polymers in high dimensions. J. Stat. Phys., 67:1009-1038, (1992).

100. T. Hara and G. Slade. Mean-field behaviour and the lace expansion. In G. Grimmett, editor, Probability and Phase Transition, Dordrecht, (1994). Kluwer.

101. T. Hara and G. Slade. The self-avoiding-walk and percolation critical points in high dimensions. Combin. Probab. Comput., 4:197-215, (1995).

102. T. Hara and G. Slade. Unpublished appendix to [101]. Available as paper 93288 at http://www.ma.utexas.edu/mp_arc. (1993).

103. T. Hara and G. Slade. The incipient infinite cluster in high-dimensional percolation. Electron. Res. Announc. Amer. Math. Soc., 4:48-55, (1998). http: //www . ams .org/era/.

104. T. Hara and G. Slade. The scaling limit of the incipient infinite cluster in highdimensional percolation. I. Critical exponents. J. Stat. Phys., 99:1075-1168, (2000).

105. T. Hara and G. Slade. The scaling limit of the incipient infinite cluster in highdimensional percolation. II. Integrated super-Brownian excursion. J. Math. Phys., 41:1244-1293, (2000).

106. T. Hara, G. Slade, and A.D. Sokal. New lower bounds on the self-avoidingwalk connective constant. J. Stat. Phys., 72:479-517, (1993). Erratum: J. Stat. Phys., 78:1187-1188, (1995).

107. T.E. Harris. Contact interactions on a lattice. Ann. Probab., 2:969-988, (1974). 
108. R. van der Hofstad. The lace expansion approach to ballistic behaviour for onedimensional weakly self-avoiding walk. Probab. Th. Rel. Fields, 119:311-349, (2001).

109. R. van der Hofstad. Infinite canonical super-Brownian motion and scaling limits. Preprint, (2004).

110. R. van der Hofstad. Spread-out oriented percolation and related models above the upper critical dimension: induction and super-processes. Preprint, (2005).

111. R. van der Hofstad, F. den Hollander, and G. Slade. The survival probability for critical spread-out oriented percolation above $4+1$ dimensions. I. Induction. In preparation.

112. R. van der Hofstad, F. den Hollander, and G. Slade. The survival probability for critical spread-out oriented percolation above $4+1$ dimensions. II. Expansion. In preparation.

113. R. van der Hofstad, F. den Hollander, and G. Slade. A new inductive approach to the lace expansion for self-avoiding walks. Probab. Th. Rel. Fields, 111:253286, (1998).

114. R. van der Hofstad, F. den Hollander, and G. Slade. Construction of the incipient infinite cluster for spread-out oriented percolation above $4+1$ dimensions. Commun. Math. Phys., 231:435-461, (2002).

115. R. van der Hofstad and A.A. Járai. The incipient infinite cluster for highdimensional unoriented percolation. J. Stat. Phys, 114:625-663, (2004).

116. R. van der Hofstad and W. König. A survey of one-dimensional random polymers. J. Stat. Phys., 103:915-944, (2001).

117. R. van der Hofstad and A. Sakai. Convergence of the critical finite-range contact process to super-Brownian motion above the upper critical dimension. In preparation.

118. R. van der Hofstad and A. Sakai. Critical points for spread-out self-avoiding walk, percolation and the contact process. Probab. Th. Rel. Fields, 132:438470, (2005).

119. R. van der Hofstad and A. Sakai. Gaussian scaling for the critical spreadout contact process above the upper critical dimension. Electron. J. Probab., 9:710-769, (2004).

120. R. van der Hofstad and G. Slade. A generalised inductive approach to the lace expansion. Probab. Th. Rel. Fields, 122:389-430, (2002).

121. R. van der Hofstad and G. Slade. Convergence of critical oriented percolation to super-Brownian motion above $4+1$ dimensions. Ann. Inst. H. Poincaré Probab. Statist., 39:415-485, (2003).

122. R. van der Hofstad and G. Slade. Asymptotic expansions in $n^{-1}$ for percolation critical values on the $n$-cube and $\mathbb{Z}^{n}$. To appear in Random Struct. Alg.

123. R. van der Hofstad and G. Slade. Expansion in $n^{-1}$ for percolation critical values on the $n$-cube and $\mathbb{Z}^{n}$ : the first three terms. To appear in Combin. Probab. Comput.

124. R. van der Hofstad and G. Slade. The lace expansion on a tree with application to networks of self-avoiding walks. Adv. Appl. Math., 30:471-528, (2003).

125. M. Holmes. Convergence of lattice trees to super-Brownian motion above the critical dimension. PhD thesis, University of British Columbia, (2005).

126. M. Holmes, A.A. Járai, A. Sakai, and G. Slade. High-dimensional graphical networks of self-avoiding walks. Canad. J. Math., 56:77-114, (2004).

127. B.D. Hughes. Random Walks and Random Environments, volume 1: Random Walks. Oxford University Press, Oxford, (1995). 
128. B.D. Hughes. Random Walks and Random Environments, volume 2: Random Environments. Oxford University Press, Oxford, (1996).

129. D. Iagolnitzer and J. Magnen. Polymers in a weak random potential in dimension four: rigorous renormalization group analysis. Commun. Math. Phys., 162:85-121, (1994).

130. D. Ioffe. Ornstein-Zernike behaviour and analyticity of shapes for self-avoiding walks on $Z^{d}$. Markov Process. Related Fields, 4:323-350, (1998).

131. E. J. Janse van Rensburg. On the number of trees in $\mathbf{Z}^{d}$. J. Phys. A: Math. Gen., 25:3523-3528, (1992).

132. E. J. Janse van Rensburg. The Statistical Mechanics of Interacting Walks, Polygons, Animals and Vesicles. Oxford University Press, Oxford, (2000).

133. E. J. Janse van Rensburg and N. Madras. A non-local Monte-Carlo algorithm for lattice trees. J. Phys. A: Math. Gen., 25:303-333, (1992).

134. S. Janson, T. Łuczak, and A. Ruciński. Random Graphs. John Wiley and Sons, New York, (2000).

135. A.A. Járai. Incipient infinite percolation clusters in 2d. Ann. Probab., 31:444485, (2003).

136. A.A. Járai. Invasion percolation and the incipient infinite cluster in $2 d$. Commun. Math. Phys., 236:311-334, (2003).

137. I. Jensen and A.J. Guttmann. Self-avoiding polygons on the square lattice. J. Phys. A: Math. Gen., 32:4867-4876, (1999).

138. T. Kennedy. Ballistic behavior in a $1 \mathrm{D}$ weakly self-avoiding walk with decaying energy penalty. J. Stat. Phys., 77:565-579, (1994).

139. T. Kennedy. Conformal invariance and stochastic Loewner evolution predictions for the 2D self-avoiding walk-Monte Carlo tests. J. Stat. Phys., 114:51$78,(2004)$.

140. H. Kesten. On the number of self-avoiding walks. II. J. Math. Phys., 5:11281137, (1964)

141. H. Kesten. Percolation Theory for Mathematicians. Birkhäuser, Boston, (1982)

142. H. Kesten. The incipient infinite cluster in two-dimensional percolation. Probab. Th. Rel. Fields, 73:369-394, (1986).

143. H. Kesten. Asymptotics in high dimensions for percolation. In G.R. Grimmett and D.J.A. Welsh, editors, Disorder in Physical Systems. Clarendon Press, Oxford, (1990).

144. K.M. Khanin, J.L. Lebowitz, A.E. Mazel, and Ya.G. Sinai. Self-avoiding walks in five or more dimensions: polymer expansion approach. Russian Math. Surveys, 50:403-434, (1995).

145. D.J. Klein. Rigorous results for branched polymer models with excluded volume. J. Chem. Phys., 75:5186-5189, (1981).

146. W. König. A central limit theorem for a one-dimensional polymer measure. Ann. Probab, 24:1012-1035, (1996).

147. G.F. Lawler. A self-avoiding random walk. Duke Math. J., 47:655-693, (1980).

148. G.F. Lawler. The infinite self-avoiding walk in high dimensions. Ann. Probab., 17:1367-1376, (1989).

149. G.F. Lawler. Intersections of Random Walks. Birkhäuser, Boston, (1991).

150. G.F. Lawler, O. Schramm, and W. Werner. On the scaling limit of planar self-avoiding walk. In Fractal geometry and applications: a jubilee of Benoît Mandelbrot, Part 2, pages 339-364, Proc. Sympos. Pure Math., 72, Part 2. Amer. Math. Soc., Providence, RI, (2004). 
151. J.-F. Le Gall. The uniform random tree in a Brownian excursion. Probab. Th. Rel. Fields, 96:369-383, (1993).

152. J.-F. Le Gall. Spatial Branching Processes, Random Snakes, and Partial Differential Equations. Birkhäuser, Basel, (1999).

153. T.M. Liggett. Interacting Particle Systems. Springer, New York, (1985).

154. T.M. Liggett. Stochastic Interacting Systems: Contact, Voter and Exclusion Processes. Springer, Berlin, (1999).

155. T.C. Lubensky and J. Isaacson. Statistics of lattice animals and dilute branched polymers. Phys. Rev., A20:2130-2146, (1979).

156. T. Łuczak. Component behavior near the critical point of the random graph process. Random Structures Algorithms, 1:287-310, (1990).

157. N. Madras. A rigorous bound on the critical exponent for the number of lattice trees, animals and polygons. J. Stat. Phys., 78:681-699, (1995).

158. N. Madras and G. Slade. The Self-Avoiding Walk. Birkhäuser, Boston, (1993).

159. N. Madras and A.D. Sokal. The pivot algorithm: A highly efficient Monte Carlo method for the self-avoiding walk. J. Stat. Phys., 50:109-186, (1988).

160. R. Meester and R. Roy. Continuum Percolation. Cambridge University Press, Cambridge, (1996).

161. M.V. Menshikov. Coincidence of critical points in percolation problems. Soviet Mathematics, Doklady, 33:856-859, (1986).

162. M.V. Menshikov, S.A. Molchanov, and A.F. Sidorenko. Percolation theory and some applications. Itogi Nauki i Tekhniki (Series of Probability Theory, Mathematical Statistics, Theoretical Cybernetics), 24:53-110, (1986). English Translation: J. of Soviet Mathematics, 42:1766-1810, (1988).

163. A.M. Nemirovsky, K.F. Freed, T. Ishinabe, and J.F. Douglas. End-to-end distance of a single self-interacting self-avoiding polymer chain: $d^{-1}$ expansion. Phys. Lett. A, 162:469-474, (1992).

164. A.M. Nemirovsky, K.F. Freed, T. Ishinabe, and J.F. Douglas. Marriage of exact enumeration and 1/d expansion methods: Lattice model of dilute polymers. $J$. Stat. Phys., 67:1083-1108, (1992).

165. B.G. Nguyen. Gap exponents for percolation processes with triangle condition. J. Stat. Phys., 49:235-243, (1987).

166. B.G. Nguyen and W-S. Yang. Triangle condition for oriented percolation in high dimensions. Ann. Probab., 21:1809-1844, (1993).

167. B.G. Nguyen and W-S. Yang. Gaussian limit for critical oriented percolation in high dimensions. J. Stat. Phys., 78:841-876, (1995).

168. B. Nienhuis. Exact critical exponents of the $O(n)$ models in two dimensions. Phys. Rev. Lett., 49:1062-1065, (1982).

169. B. Nienhuis. Critical behavior of two-dimensional spin models and charge asymmetry in the Coulomb gas. J. Stat. Phys., 34:731-761, (1984).

170. B. Nienhuis. Coulomb gas formulation of two-dimensional phase transitions. In C. Domb and J.L. Lebowitz, editors, Phase Transitions and Critical Phenomena, volume 11, New York, (1987). Academic Press.

171. J. Noonan. New upper bounds on the connective constants of self-avoiding walks. J. Stat. Phys., 91:871-888, (1998).

172. S.P. Obukhov. The problem of directed percolation. Physica, 101A:145-155, (1980).

173. A.L. Owczarek and T. Prellberg. Scaling of self-avoiding walks in high dimensions. J. Phys. A: Math. Gen., 34:5773-5780, (2001). 
174. A.L. Owczarek and T. Prellberg. Monte Carlo investigations of lattice models of polymer collapse in five dimensions. Internat. J. Modern Phys. C, 14:621633, (2003).

175. G. Parisi and N. Sourlas. Critical behavior of branched polymers and the Lee-Yang edge singularity. Phys. Rev. Lett., 46:871-874, (1981).

176. M.D. Penrose. Self-avoiding walks and trees in spread-out lattices. J. Stat. Phys., 77:3-15, (1994).

177. E. Perkins. Dawson-Watanabe superprocesses and measure-valued diffusions. In P.L. Bernard, editor, Lectures on Probability Theory and Statistics. Ecole d'Eté de Probabilités de Saint-Flour XXIX-1999, pages 125-329, Berlin, (2002). Springer. Lecture Notes in Mathematics \#1781.

178. E. Perkins. Super-Brownian motion and critical spatial stochastic systems. Canad. Math. Bull., 47:280-297, (2004).

179. A. Sakai. Mean-field critical behavior for the contact process. J. Stat. Phys., 104:111-143, (2001).

180. A. Sakai. Hyperscaling inequalities for the contact process and oriented percolation. J. Stat. Phys., 106:201-211, (2002).

181. A. Sakai. Lace expansion for the Ising model. In preparation.

182. L. Serlet. Super-Brownian motion conditioned on the total mass. Preprint, (2005).

183. G. Slade. The diffusion of self-avoiding random walk in high dimensions. Commun. Math. Phys., 110:661-683, (1987).

184. G. Slade. Convergence of self-avoiding random walk to Brownian motion in high dimensions. J. Phys. A: Math. Gen., 21:L417-L420, (1988).

185. G. Slade. The scaling limit of self-avoiding random walk in high dimensions. Ann. Probab., 17:91-107, (1989).

186. G. Slade. The lace expansion and the upper critical dimension for percolation. Lectures in Applied Mathematics, 27:53-63, (1991). (Mathematics of Random Media, eds. W.E. Kohler and B.S. White, A.M.S., Providence).

187. G. Slade. Bounds on the self-avoiding-walk connective constant. Journal of Fourier Analysis and Applications, Special Issue: Proceedings of the Conference in Honor of Jean-Pierre Kahane (Orsay, June 28 - July 3 1993):525-533, (1995).

188. G. Slade. Lattice trees, percolation and super-Brownian motion. In M. Bramson and R. Durrett, editors, Perplexing Problems in Probability: Festschrift in Honor of Harry Kesten, Basel, (1999). Birkhäuser.

189. G. Slade. Scaling limits and super-Brownian motion. Notices A.M.S., 49(9):1056-1067, (2002).

190. S. Smirnov. Critical percolation in the plane. I. Conformal invariance and Cardy's formula. II. Continuum scaling limit. Preprint, (2001).

191. S. Smirnov and W. Werner. Critical exponents for two-dimensional percolation. Math. Res. Lett., 8:729-744, (2001).

192. A.D. Sokal. A rigorous inequality for the specific heat of an Ising or $\varphi^{4}$ ferromagnet. Phys. Lett., 71A:451-453, (1979).

193. C.E. Soteros. Private communication.

194. C.E. Soteros, D.W. Sumners, and S.G. Whittington. Entanglement complexity of graphs in $Z^{3}$. Math. Proc. Camb. Phil. Soc., 111:75-91, (1992).

195. F. Spitzer. Principles of Random Walk. Springer, New York, 2nd edition, (1976).

196. R.P. Stanley. Enumerative Combinatorics, volume 2. Cambridge University Press, Cambridge, (1999). 
197. D. Stauffer and A. Aharony. Introduction to Percolation Theory. Taylor and Francis, London, 2nd edition, (1992).

198. H. Tanemura. Critical behavior for a continuum percolation model. In S. Watanabe, M. Fukushima, Yu.V. Prohorov, and A.N. Shiryaev, editors, Probability theory and mathematical statistics (Tokyo 1995), pages 485-495, River Edge, N.J., (1996). World Sci. Publishing.

199. H. Tasaki. Stochastic geometric methods in statistical physics and field theories. PhD thesis, University of Tokyo, (1986).

200. H. Tasaki. Hyperscaling inequalities for percolation. Commun. Math. Phys. 113:49-65, (1987).

201. H. Tasaki and T. Hara. Critical behaviour in a system of branched polymers. Prog. Theor. Phys. Suppl., 92:14-25, (1987).

202. G. Toulouse. Perspectives from the theory of phase transitions. Nuovo Cimento, 23B:234-240, (1974).

203. K. Uchiyama. Green's functions for random walks on $\mathbb{Z}^{N}$. Proc. London Math. Soc., 77:215-240, (1998).

204. D. Ueltschi. A self-avoiding walk with attractive interactions. Probab. Th. Rel. Fields, 124:189-203, (2002).

205. C. Vanderzande. Lattice Models of Polymers. Cambridge University Press, Cambridge, (1998).

206. W. Werner. Random planar curves and Schramm-Loewner evolutions. In J. Picard, editor, Lectures on Probability Theory and Statistics. Ecole d'Eté de Probabilités de Saint-Flour XXXII-2002. Springer, Berlin (2004). Lecture Notes in Mathematics \#1840.

207. W-S. Yang and D. Klein. A note on the critical dimension for weakly selfavoiding walks. Prob. Th. and Rel. Fields, 79:99-114, (1988).

208. W-S. Yang and B. Nguyen. Gaussian limit for oriented percolation in high dimensions. In G.J. Morrow and W-S. Yang, editors, Probability Models in Mathematical Physics, Teaneck, NJ, (1991). World Scientific.

209. W-S. Yang and A. Zeleke. Expansion methods and scaling limits above critical dimensions. Taiwanese J. Math., 3:425-474, (1999).

210. W-S. Yang and Y. Zhang. A note on differentiability of the cluster density for independent percolation in high dimensions. J. Stat. Phys., 66:1123-1138, (1992).

211. D. Zeilberger. The abstract lace expansion. Adv. Appl. Math., 19:355-359, (1997). 